\title{
The Psychopharmacology of Obsessive-Compulsive Disorder: A Preclinical Roadmap
}

\author{
Henry Szechtman, Brian H. Harvey, Erik Z. Woody, and Kurt Leroy Hoffman
}

Department of Psychiatry and Behavioural Neurosciences, McMaster University, Hamilton, Ontario, Canada (H.S.); SAMRC Unit on Risk Resilience in Mental Disorders, Department of Psychiatry, University of Cape Town, and Center of Excellence for Pharmaceutical Sciences, School of Pharmacy, North-West University (Potchefstroom Campus), Potchefstroom, South Africa (B.H.H.); Department of Psychology, University of Waterloo, Waterloo, Ontario, Canada (E.Z.W.); and Centro de Investigación en Reproducción Animal, CINVESTAVUniversidad Autónoma de Tlaxcala, Tlaxcala, Mexico (K.L.H.)

Abstract........................................................ 82

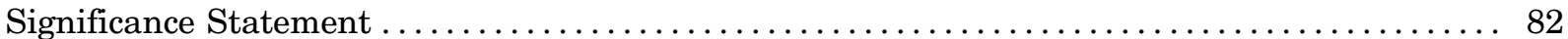

I. Overview. .......................................................... 83

II. Currently Accepted Practice and Understanding of Obsessive-Compulsive Disorder ........ 83

A. Current Understanding of What Obsessive-Compulsive Disorder Is . . . . . . . . . . . . 83

1. Diagnosis and Description of Obsessive-Compulsive Disorder ................ 83

2. Differential Diagnosis ......................................... 86

B. Pharmacotherapy for Obsessive-Compulsive Disorder in Clinical Practice . . . . . . . . . . 86

1. Monotherapy with Serotonin Reuptake Inhibitors ...................... 86

2. Augmentation of Selective Serotonin Reuptake Inhibitor Pharmacotherapy with

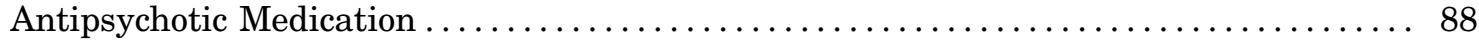

3. Augmentation of Selective Serotonin Reuptake Inhibitor Pharmacotherapy with Psychologic Treatment ......................................... 89

C. Neuromodulatory Therapies for Obsessive-Compulsive Disorder $\ldots \ldots \ldots \ldots \ldots \ldots \ldots \ldots$. 91

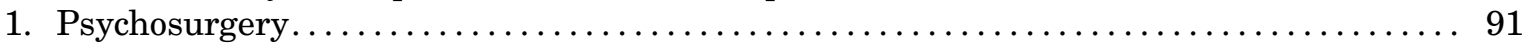

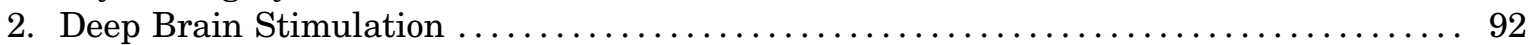

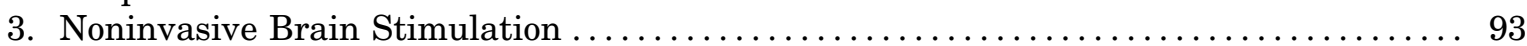

a. Repetitive Transcranial Magnetic Stimulation ........................ 93

b. Transcranial Direct Current Stimulation ................................. 94

4. Neuromodulatory Therapy Combined with Other Forms of Obsessive-Compulsive

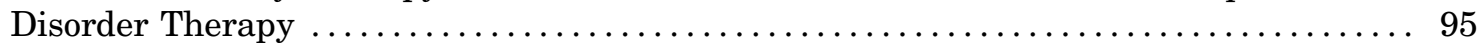

D. Psychologic Treatments for Obsessive-Compulsive Disorder. . . . . . . . . . . . . . . 95

1. Behavior Therapy ............................................ 95

a. Historical Roots of Exposure and Response/Ritual Prevention Therapy .......... 95

b. Description of Exposure and Response/Ritual Prevention Therapy............. 96

2. Cognitive Therapy........................................... 96

3. Efficacy of Psychologic Intervention............................. 97

a. Exposure and Response Prevention, Cognitive Therapy, or Cognitive-Behavioral Therapy as Monotherapy......................................

Address correspondence to: Dr. Henry Szechtman, Department of Psychiatry and Behavioural Neurosciences, McMaster University, MUMC, 4N82, 1280 Main St. West, Hamilton, ON L8S 4K1, Canada. E-mail: szechtma@mcmaster.ca

B.H.H. is supported by the South African Medical Research Council. Parts of the work presented in this review were funded by the National Research Foundation (NRF) [Grants CPRR14080485951 and 77323 (to B.H.H.)] and an unrestricted grant by Servier Laboratories. Any opinions, findings, and conclusions or recommendations are those of the authors, and the NRF accepts no liability whatsoever in this regard. Servier did not provide any input on this manuscript, and opinions and/or recommendations expressed in this article are those of the authors and not those of Servier. B.H.H. declares that over the past 3 years, he has participated in advisory boards and received honoraria from Servier and has received research funding from Servier and Lundbeck. Except for income from the primary employer and research funding to B.H.H. from the above-mentioned organizations and agencies, no financial support or compensation has been received from any individual or corporate entity over the past 3 years for research or professional services, and there are no personal financial holdings that could be perceived as constituting a potential conflict of interest.

https://doi.org/10.1124/pr.119.017772. 


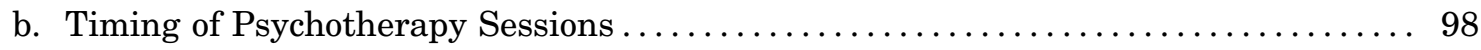

4. Efficacy of Cognitive-Behavioral Therapy with or without Pharmacotherapy ........ 99

5. Mechanisms of Cognitive-Behavioral Therapy Therapeutic Effect on the Obsessive-

Compulsive Disorder Condition ...................................... 100

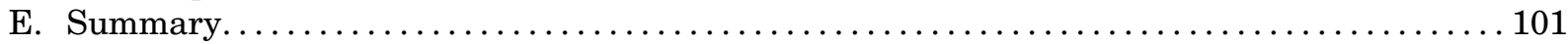

III. Current Research on the Neurobiology of Obsessive-Compulsive Disorder............... 102

A. Probing the Role of Neurotransmitters in Obsessive-Compulsive Disorder............ 102

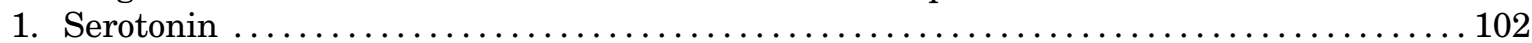

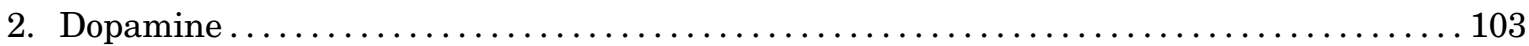

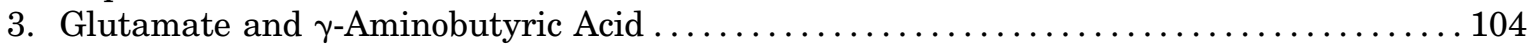

B. Probing the Role of Other Endogenous Processes in Obsessive-Compulsive Disorder...... 105

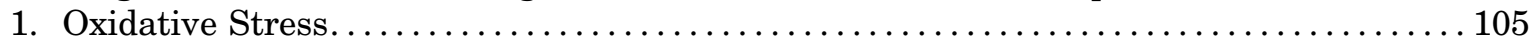

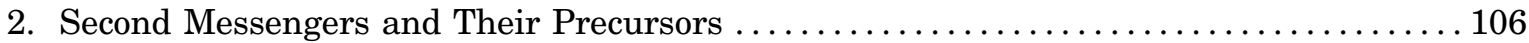

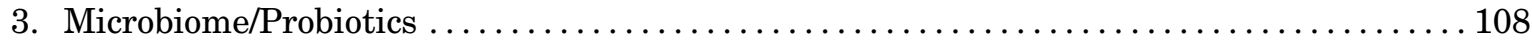

4. Neurosteroids and Neuropeptides..................................... 109

5. Neurotrophic Factors . ........................................ 110

C. Probing the Neuroanatomical Location of the Neurobiological Fault in Obsessive-

Compulsive Disorder . ....................................... 110

D. Probing Etiology for the Neurobiological Fault in Obsessive-Compulsive Disorder ........ 111

1. Genetics of Obsessive-Compulsive Disorder ........................... 111

a. Human Studies of the Genetic Correlates of Obsessive-Compulsive Disorder....... 111

b. Animal Models Based on Gene Association Findings for Obsessive-Compulsive

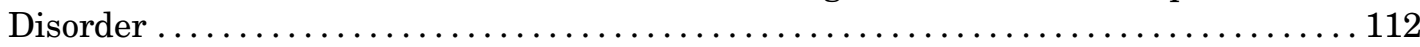

i. Solute Carrier Family 1 Member 1/Sodium-Dependent Glutamate/Aspartate

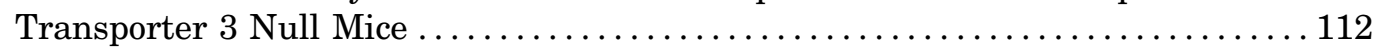

ii. Sap90/Postsynaptic Density Protein-95-Associated Protein 3 Null Mice ........ 112

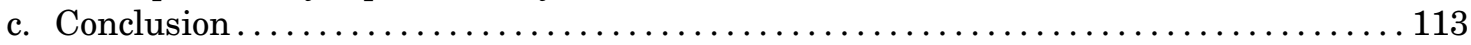

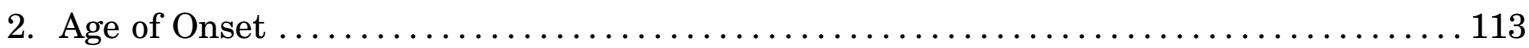

3. Hormonal Correlates of Obsessive-Compulsive Disorder $\ldots \ldots \ldots \ldots \ldots \ldots \ldots \ldots \ldots \ldots \ldots$

4. Immune System Correlates of Obsessive-Compulsive Disorder. . . . . . . . . . . . . . . 114

a. Infection and the Immune System in Obsessive-Compulsive Disorder . . . . . . . . 114

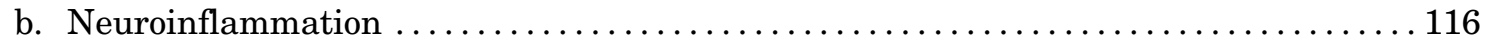

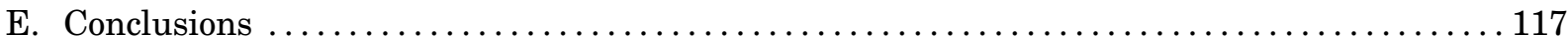

IV. Toward a New Perspective on Preclinical Research............................ 117

A. Alternative Frameworks for Research on Obsessive-Compulsive Disorder ............. 117

1. The Diagnostic and Statistical Manual of Mental Disorders versus the Research

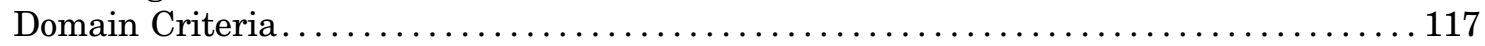

2. An Adaptationist Reorientation of the Research Domain Criteria Approach for

Obsessive-Compulsive Disorder Research .............................. 118

B. The Security Motivation System ................................... 120

1. Obsessive-Compulsive Disorder as the Dysfunction of a Normal Motivational

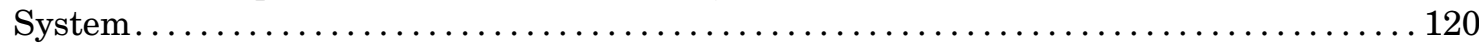

\begin{abstract}
ABBREVIATIONS: 5-HT, serotonin; AC, adenylate cyclase; ACC, anterior cingulate cortex; ACTH, adrenocorticotropic hormone; ADHD, attention-deficit/hyperactivity disorder; ALIC, anterior limb of the internal capsule; ASD, autism spectrum disorder; BDNF, brain-derived neurotrophic factor; BDV, Borna disease virus; BG-TH-CTX, basal ganglia-thalamus-cortex; BST, bed nucleus of the stria terminalis; CBT, cognitive-behavioral therapy; CPD 1, (3S)-3-methyl-1-[4-(trifluoromethyl)-7-benzofuranyl]-piperazine; CREB, cAMP response elementbinding protein; CSTC, cortico-striato-thalamo-cortical; CT, cognitive therapy; DBS, deep brain stimulation; DSM, Diagnostic and Statistical Manual of Mental Disorders; EAAT, excitatory amino acid transporter; ERP, exposure and response prevention; ES, effect size; GABHS, group A $\beta$-hemolytic Streptococcus pyogenes; GSH, glutathione; GWAS, genome-wide association study; HPA, hypothalamic-pituitary-adrenal; IL, interleukin; mCPP, $m$-chlorophenylpiperazine; MI, myo-inositol; NAC, $N$-acetyl cysteine; NAD, nicotinamide adenine dinucleotide; NMDA, $N$-methyl-D-aspartate; NO, nitric oxide; NOS, nitric oxide synthase; OC, obsessive-compulsive; OCD, obsessive-compulsive disorder; OFC, orbitofrontal cortex; PANDAS, pediatric autoimmune neuropsychiatric disorders associated with streptococcal infections; PANS, pediatric acute-onset neuropsychiatric syndrome; PARP, poly(ADP-ribose) polymerase; PD, Parkinson disease; PDE, phosphodiesterase; PI, phosphoinositide; PTSD, post-traumatic stress disorder; QS, quinpirole sensitization; RDoC, Research Domain Criteria; rTMS, repetitive transcranial magnetic stimulation; SA, signal attenuation; SCN, suprachiasmatic nucleus; SMS, security motivation system; SRI, serotonin reuptake inhibitor; SSRI, selective serotonin reuptake inhibitor; STN, subthalamic nucleus; TCA, tricyclic antidepressant; tDCS, transcranial direct current stimulation; TMS, transcranial magnetic stimulation; TNF, tumor necrosis factor; TRYCAT, tryptophan catabolic; TS, Gille de la Tourette syndrome; YBOCS, Yale-Brown Obsessive Compulsive Scale.
\end{abstract}


2. The Systems-Design Approach to Obsessive-Compulsive Disorder and the Main Themes of the Research Domain Criteria ............................... 121

C. Key Aspects of the Security Motivation System Theory of Obsessive-Compulsive

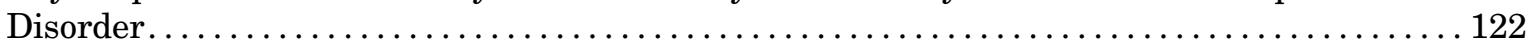

V. Toward a New Perspective on Psychopharmacological Research on Obsessive-Compulsive

Disorder ........................................................... 125

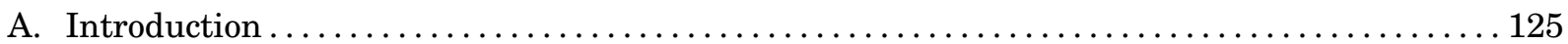

B. Enhancing Infrastructure to Boost Processing Power of the Brain . . . . . . . . . . . 125

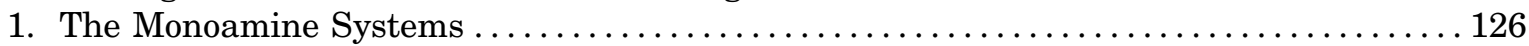

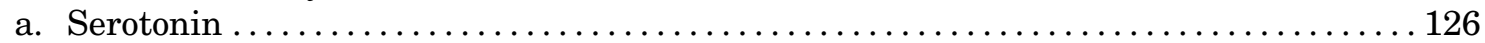

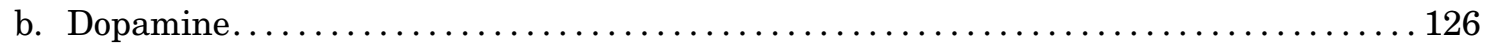

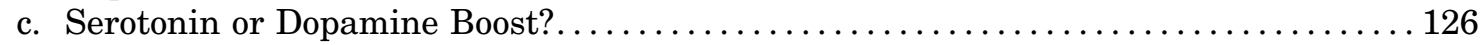

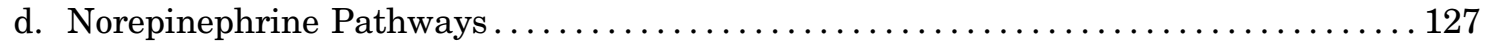

2. Circadian Rhythm Facility ........................................ 127

a. Importance of Circadian Rhythms for Optimizing Brain Resources . . . . . . . . . 127

b. Pathways by Which Nonphotic Zeitgebers May Boost Circadian Infrastructure and Thus Ameliorate Obsessive-Compulsive Disorder .................... 128

3. Nicotinamide Adenine Dinucleotide Metabolism Infrastructure .................. 129

a. Importance of Nicotinamide Adenine Dinucleotide in Living Cells . . . . . . . . . . . 129

b. Redox Reactions ............................................... 129

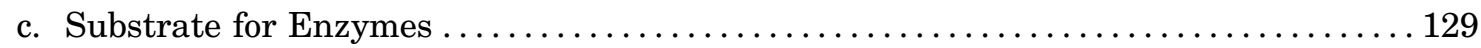

d. Biosynthesis and Strategies to Boost Nicotinamide Adenine Dinucleotide......... 129

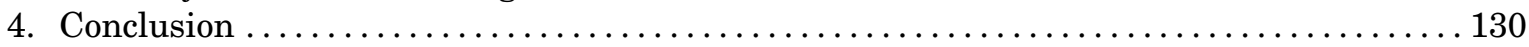

C. Facilitating Relearning to Promote Neural Plasticity $\ldots \ldots \ldots \ldots \ldots \ldots \ldots \ldots \ldots \ldots \ldots 130$

D. Fixing a Weak Link: Targeting Specific Pathways of the Security Motivation System

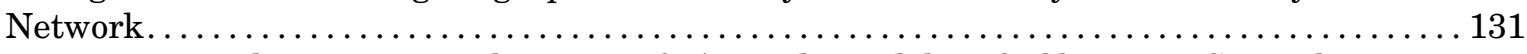

1. Motivational Reconceptualization of Animal Models of Obsessive-Compulsive

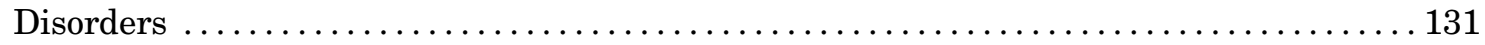

2. Neurochemistry of the Negative Feedback "Satiety" Signal(s) . . . . . . . . . . . . 133

a. Serotonin Receptors as a Component of the Negative Feedback ................ 133

b. Microglia "Off" Signals as a Component of the Negative Feedback............... 134

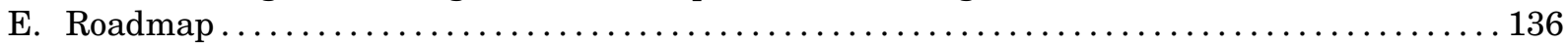

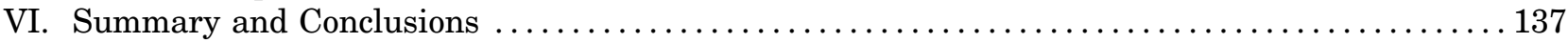

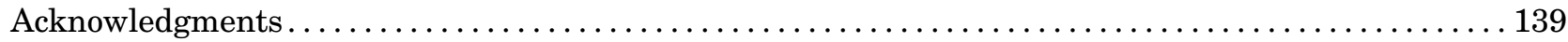

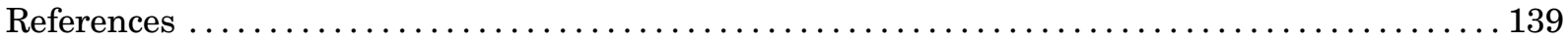

\begin{abstract}
This review evaluates current knowledge about obsessive-compulsive disorder (OCD), with the goal of providing a roadmap for future directions in research on the psychopharmacology of the disorder. It first addresses issues in the description and diagnosis of OCD, including the structure, measurement, and appropriate description of the disorder and issues of differential diagnosis. Current pharmacotherapies for OCD are then reviewed, including monotherapy with serotonin reuptake inhibitors and augmentation with antipsychotic medication and with psychologic treatment. Neuromodulatory therapies for OCD are also described, including psychosurgery, deep brain stimulation, and noninvasive brain stimulation. Psychotherapies for OCD are then reviewed, focusing on behavior therapy, including exposure and response prevention and cognitive therapy, and the efficacy of these interventions is discussed, touching on issues such as the timing of sessions, the adjunctive role of pharmacotherapy, and the underlying mechanisms. Next, current research on the neurobiology of OCD is examined, including work probing the role of various neurotransmitters and other endogenous processes
\end{abstract}

and etiology as clues to the neurobiological fault that may underlie OCD. A new perspective on preclinical research is advanced, using the Research Domain Criteria to propose an adaptationist viewpoint that regards OCD as the dysfunction of a normal motivational system. A systems-design approach introduces the security motivation system (SMS) theory of OCD as a framework for research. Finally, a new perspective on psychopharmacological research for OCD is advanced, exploring three approaches: boosting infrastructure facilities of the brain, facilitating psychotherapeutic relearning, and targeting specific pathways of the SMS network to fix deficient SMS shut-down processes.

Significance Statement_-A significant proportion of patients with obsessive-compulsive disorder (OCD) do not achieve remission with current treatments, indicating the need for innovations in psychopharmacology for the disorder. OCD may be conceptualized as the dysfunction of a normal, special motivation system that evolved to manage the prospect of potential danger. This perspective, together with a wide-ranging 
review of the literature, suggests novel directions for psychopharmacological research, including boosting support systems of the brain, facilitating relearning that occurs in psychotherapy, and targeting specific pathways in the brain that provide deficient stopping processes in OCD.

\section{Overview}

The last 3 decades have seen a remarkable outpouring of clinical and laboratory research on obsessive-compulsive disorder (OCD). An impressive indication of the success of this work is that OCD, long regarded as puzzling and peculiarly resistant to treatment, has yielded major advances in both the understanding of the disorder and its pharmacological and psychotherapeutic treatment. Nonetheless, as many as half of all patients with OCD do not attain remission with current treatments, suggesting the considerable potential for further innovations in psychopharmacology for the disorder. In addition, the scientific literature relevant to OCD, which provides the foundation from which such innovations are likely to arise, has become voluminous and very diverse, involving a huge range of methodologies: from clinical trials of many different kinds of proposed treatments, to laboratory work probing a wide range of possible biochemical, neuroanatomical, and genetic aspects of the underlying neurobiology of the disorder, to research using animal models that offer unique windows for simulating and probing the nature of the disorder. To some extent, researchers pursuing these various lines of investigation may tend to work in comparative isolation from one another, with somewhat limited knowledge of parallel developments that could provide productive insights for them. Hence, the purpose of this review is to provide a reasonably comprehensive evaluation of current scientific knowledge about OCD and in doing so to highlight promising leads for psychopharmacological research on the disorder, thus providing a "roadmap" for future research directions.

In section II, we first consider the definition and diagnosis of OCD, including issues concerning its structure, measurement, and differential diagnosis. Next, we review the range of current pharmacotherapies for OCD, including monotherapy with serotonin reuptake inhibitors (SRIs) and augmentation using antipsychotic medications and psychotherapy. We also consider neuromodulatory therapies for OCD, including psychosurgery, deep brain stimulation (DBS) and noninvasive brain stimulation techniques, repetitive transcranial magnetic stimulation (rTMS), and transcranial direct current stimulation (tDCS). We then survey psychotherapies for OCD, including behavior therapy, using techniques of exposure and response prevention (ERP) and cognitive therapy (CT), and we address treatment issues such as the timing of sessions, the possible adjunctive role of pharmacotherapy, and the hypothesized underlying mechanisms. We conclude this section by drawing attention to important conceptual problems in this clinical research and limitations of the results of clinical treatment studies for inferring the etiology and pathophysiology of the disorder.

In section III, we address the current state of research examining the neurobiology of OCD. We begin with work probing the possible role of several different neurotransmitters in the disorder, including serotonin (5-HT), dopamine, glutamate, and GABA. We also review a range of other endogenous processes that may be implicated in OCD, including oxidative stress, second messengers, the microbiome, neurosteroids and neuropeptides, and neurotrophic factors. Next, we discuss research on the etiology of OCD, including genetics, hormonal factors, and immune system correlates. Throughout, we evaluate these lines of research for clues to the neurobiological fault that may underlie OCD. However, we also point out that this body of very diverse, sometimes contradictory, findings relating to OCD seems urgently in need of a unifying theoretical and conceptual framework that could be used to integrate and organize them. Indeed, taking steps toward achieving this task is the objective of sections IV and V.

In section IV, we advance a new perspective on preclinical research into OCD. Using the Research Domain Criteria (RDoC) as a starting point, we propose an adaptationist viewpoint that considers OCD as the dysfunctional dynamics of a normal motivational system present in all people. A systems-design approach then introduces the security motivation system (SMS) theory of OCD as a broad framework for future research, paying particular attention to ways in which this theory casts some of the key phenomena of OCD in a new light.

Finally, using the SMS theory as a guiding framework, we provide a novel perspective on alternative ways to conceptualize potential targets for pharmacological intervention in OCD in section V. In particular, we distinguish and explore three different approaches: 1) boosting infrastructure facilities (or support systems) of the brain; 2) facilitating relearning, including that which occurs in psychotherapy; and 3) targeting specific pathways of the SMS network to fix deficient stopping processes in OCD. Throughout this discussion, we highlight some new ideas for future psychopharmacological research on OCD.

\section{Currently Accepted Practice and Understanding of Obsessive- Compulsive Disorder}

\section{A. Current Understanding of What Obsessive-Compulsive Disorder Is}

1. Diagnosis and Description of Obsessive-Compulsive Disorder. There is currently no biologic test that identifies OCD. Moreover, the etiology and pathophysiology of 
this disorder are unknown. Hence, the diagnosis of OCD is made solely from evidence that a particular behavioral profile exists, a profile that defines what constitutes OCD. This profile is described in the Diagnostic and Statistical Manual of Mental Disorders (DSM; Chapter 13 in American Psychiatric Association, 2013). According to the DSM-5 manual, "OCD is characterized by the presence of obsessions and/or compulsions. Obsessions are recurrent and persistent thoughts, urges, or images that are experienced as intrusive and unwanted, whereas compulsions are repetitive behaviors or mental acts that an individual feels driven to perform in response to an obsession or according to rules that must be applied rigidly" (p. 235). Additional information regarding the OCD behavioral profile is provided in Table 1, which contains the DSM-5 criteria for a diagnosis of OCD (American Psychiatric Association, 2013).

In a comprehensive phenomenological analysis of obsessional experience and compulsive behavior, Reed (1985) found that the obsessions of individuals with OCD were characterized by a preoccupation with thoughts of the following: 1) unworthiness, either with reference to perceived social norms or the individual's own standards; 2) one's bodily functions; 3 ) dirt and the spread of disease to oneself; and 4) harming a friend or relative and being responsible for the imagined event. Moreover, Reed (1985) found that the compulsive behaviors of individuals with OCD were characterized by the following: 1) excessive checking activities, which include repeated redoings of actions supposedly related to security, orderliness, or accuracy; 2) avoidance behaviors, which are "activities engaged in to avoid feared objects, places, or situations" (p. 37); and 3) washing and cleaning, generally of hands but sometimes also compulsive washing of clothes, teeth cleaning, or the cleaning of possessions or parts of the home. An individual with OCD could be preoccupied with more than one obsession and/or compulsion. Others have noted that the two most salient features of obsessions are doubts and indecision, and that the motor acts of checking and washing are the two most common compulsive behaviors (Rasmussen and Eisen, 1992).

It had been argued that to reveal the mechanisms of OCD, and indeed for a fuller clinical understanding of OCD, it may be more useful to focus on the structure or form of OCD symptoms rather than their content (Reed, 1985). Reed (1985) identified three essential features of obsessions and compulsions: 1) certain types of ideas (obsessions) and behaviors (compulsions) preoccupy the individual excessively and this preoccupation interferes with normal daily function; 2) the preoccupation gives no pleasure to the individual but the subject feels driven to be so engaged; and 3) the person acknowledges that the preoccupation may appear to others as disconnected from objective reality. As can be seen, this conceptualization of the form of OCD is consistent with a reading in

TABLE 1

DSM-5 diagnostic criteria for OCD

Diagnostic Criteria 300.3 (F42.2 F42)

A. Presence of obsessions, compulsions, or both:

Obsessions are defined by (1) and (2):

(1) Recurrent and persistent thoughts, urges, or images that are experienced, at some time during the disturbance, as intrusive and unwanted, and that in most individuals cause marked anxiety or distress.

(2) The individual attempts to ignore or suppress such thoughts, urges, or images, or to neutralize them with some other thought or action (i.e., by performing a compulsion).

Compulsions are defined by (1) and (2):

(1) Repetitive behaviors (e.g., hand washing, ordering, checking) or mental acts (e.g., praying, counting, repeating words silently) that the individual feels driven to perform in response to an obsession or according to rules that must be applied rigidly.

(2) The behaviors or mental acts are aimed at preventing or reducing anxiety or distress, or preventing some dreaded event or situation; however, these behaviors or mental acts are not connected in a realistic way with what they are designed to neutralize or prevent, or are clearly excessive.

Note: Young children may not be able to articulate the aims of these behaviors or mental acts.

B. The obsessions or compulsions are time-consuming (e.g., take more than 1 hour per day) or cause clinically significant distress or impairment in social, occupational, or other important areas of functioning.

C. The obsessive-compulsive symptoms are not attributable to the physiologic effects of a substance (e.g., a drug of abuse, a medication) or another medical condition.

D. The disturbance is not better explained by the symptoms of another mental disorder (e.g., excessive worries, as in generalized anxiety disorder; preoccupation with appearance, as in body dysmorphic disorder; difficulty discarding or parting with possessions, as in hoarding disorder; hair pulling, as in trichotillomania [hair-pulling disorder]; skin picking, as in excoriation [skin-picking] disorder; stereotypies, as in stereotypic movement disorder; ritualized eating behavior, as in eating disorders; preoccupation with substances or gambling, as in substance-related and addictive disorders; preoccupation with having an illness, as in illness anxiety disorder; sexual urges or fantasies, as in paraphilic disorders; impulses, as in disruptive, impulse-control, and conduct disorders; guilty ruminations, as in major depressive disorder; thought insertion or delusional preoccupations, as in schizophrenia spectrum and other psychotic disorders; or repetitive patterns of behavior, as in autism spectrum disorder).

Specify if:

- With good or fair insight: The individual recognizes that obsessive-compulsive disorder beliefs are definitely or probably not true or that they may or may not be true.

- With poor insight: The individual thinks obsessive-compulsive disorder beliefs are probably true.

- With absent insight/delusional beliefs: The individual is completely convinced that obsessive-compulsive disorder beliefs are true. Specify if:

- Tic-related: The individual has a current or past history of a tic disorder. 
Table 1 of diagnostic criteria A and B and, to some extent, the "insight" specifier.

Interestingly, the widely used instrument in OCD research studies, the Yale-Brown Obsessive Compulsive Scale (YBOCS; Goodman et al., 1989a,b), fully captures the first two of these OCD features (Reed, 1985). In fact, the YBOCS was specifically designed as a "measure of the severity of symptoms of obsessivecompulsive disorder that is not influenced by the type of obsessions or compulsions present" (Goodman et al., $1989 b$, p. 1006). The YBOCS quantifies features of the preoccupation by asking the individual to indicate the following, separately for obsessions and for compulsions (on a scale from 0 to 4): 1) time occupied by obsessive thoughts and compulsions $(0=$ none, $4=$ more than 8 hours/day); 2) amount of interference with daily function from obsessions and compulsions $(0=$ none, 4 = incapacitating); 3) intensity of anxiety if prevented from engagement in obsessions and compulsions $(0=$ none, 4 = incapacitating); 4) amount of effort in resisting obsessions and compulsions $(0=$ always try to resist, $4=$ yield completely and willingly); and 5) degree of control over obsessions and compulsions $(0=$ complete control, $4=$ rarely able to exert even momentary control). These 10 items yield a total score from 0 to 40 and thereby provide a quantitative index of the "preoccupation" that characterizes the structure of OCD symptoms. YBOCS score change as a function of treatment is the most commonly used outcome variable in meta-analysis reviews evaluating the effects of interventions for OCD.

The YBOCS proved clinically useful in studies of OCD because it captured well the salient structure of the OCD phenotype contained in the diagnostic criteria A and B (Table 1). However, the YBOCS does not capture the third component of the form of OCD symptoms: namely, acknowledgment by the patient that to others the OCD preoccupation may appear as disconnected from objective reality (Reed, 1985). This component of the form of OCD symptoms reflects the clinical observation that in OCD there is no confusion or disconnection from reality. Note that the stated property is that the individual realizes that "others" may see the preoccupation as disconnected from objective reality-and not that the person feels or sees it this way personally, from his or her own perspective. However, it is the latter that is assessed in the DSM-5, where clinicians rate ("specify") if the individual recognizes the senselessness of the preoccupation-that is, whether the "individual recognizes that obsessive-compulsive disorder beliefs are definitely or probably not true or that they may or may not be true" (Table 1). Rather than assessing the link to reality by asking the individual to take the perspective of an external observer, in the DSM-5, the individual is considered to have "good or fair insight" if the individual considers that his or her preoccupation has no basis in reality ("may not be true") and as having "absent insight/delusional beliefs" if the individual sees his or her preoccupation as a measured response to external circumstances (i.e., the individual "is completely convinced that obsessive-compulsive disorder beliefs are true"; Table 1). It would appear therefore that although the YBOCS does not assess the individual's tie to reality, the DSM-5 diagnostic specifier for "insight" imputes in OCD a distortion of reality (given that both "good" and "absent" insights deem the preoccupation as disconnected from external reality).

However, a distortion of reality is not a characteristic of OCD and this is recognized explicitly in the DSM-5 diagnostic criteria (criterion $\mathrm{D}$ in Table 1), in which a distortion of reality such as "thought insertion or delusional preoccupations" is used to differentiate OCD from "schizophrenia spectrum and other psychotic disorders." So why is there this inconsistency in the DSM-5 diagnostic criteria for OCD? As just suggested, it may be related to the shift in the way the link to reality is probed in the DSM-5. Specifically, rather than being asked to take on the perspective of an objective observer, the individual is asked whether subjectively there is no rationale for the preoccupation, which is contrary to the human cognitive disposition to intuit reasons for one's own actions (Mercier and Sperber, 2017). Consequently, the "insight" specifier deems a priori OCD preoccupations as senseless and disconnected from reality.

Even though ostensibly the OCD "diagnostic criteria are merely descriptive, rather than theory-based or etiologically based" (Abramowitz, 2018, p. 4), in fact the presentation of the OCD profile in the DSM-5 is infused with implicit psychologic theories. One prominent theory holds that thoughts drive actions and the two are functionally linked. This psychologic theory is exemplified in the definition of compulsion as performed "in response to an obsession" and in the claim of a meaningful functional relationship between obsessions and compulsions in that obsessions "in most individuals cause marked anxiety or distress" and compulsions "are aimed at preventing or reducing anxiety or distress" (criterion A in Table 1). Clearly, such diagnostic criteria are not purely "descriptive" of OCD symptoms but also contain a theoretical formulation of the mechanisms for the presentation of the OCD profile.

That a theoretical perspective played a role in the construction of the diagnostic criteria is evident, for example, in the report of a workgroup on DSM-5 (Leckman et al., 2010). Among the issues considered in this report was the rationale for separate definitions for obsessions and compulsions in the DSM-5 versus having a combined definition as in the tenth revision of the International Statistical Classification of Diseases and Related Health Problems. Arguments to retain separate definitions in the DSM-5 included the theoretical framework that the distinction between obsessions 
and compulsions is "central to the cognitive-behavioral therapy (CBT) approach to the conceptualization and treatment of OCD" and the "utility of emphasizing a functional relationship between obsessions and compulsions," as this "provides an accurate conceptualization of the nature of OCD symptoms" (Leckman et al., 2010, p. 510).

Unfortunately, it is not justified to embed a particular theoretical perspective into a phenomenological description of OCD symptoms. This hinders scientific research into mechanisms of the disorder because the description of the clinical phenomenon is confounded with a hidden theoretical framework, hampering alternate lines of investigation. Hence, it is more generative for research into mechanisms of OCD to describe the disorder in terms of the three-point depiction of the structure of OCD noted earlier (Reed, 1985) and to use the YBOCS as a measurement of OCD (with the proviso for the need of a separate assessment of link to reality). Moreover, as discussed in more detail later, this atheoretical, descriptive approach also provides a strong rationale for preclinical preparations to model the structure of OCD symptoms, enabling experimental studies of OCD mechanisms in animal models.

2. Differential Diagnosis. For the diagnosis of OCD, criterion $\mathrm{C}$ states: "obsessive-compulsive symptoms are not attributable to the physiological effects of a substance (e.g., a drug of abuse, a medication) or another medical condition" (Table 1). That is, if it is judged that "obsessions and compulsions are all due to the direct physiological effects of a general medical condition," then the presence of such an "etiological medical condition" entails the diagnosis not of OCD but "Obsessive-Compulsive and Related Disorder Due to Another Medical Condition" (see Table 3.6.1 in First, 2013). Similarly, "OCD is not diagnosed if the obsessions and compulsions are all due to the direct physiological effects of a substance (including medication)" and instead the diagnosis of "Substance/MedicationInduced Obsessive-Compulsive and Related Disorder" is made (see Table 3.6.1 in First, 2013).

The above differential diagnosis should not be interpreted as indicating that another disease exists with a pathophysiology distinctly different from the pathophysiology of OCD diagnosed solely from behavioral evidence. Rather, the differential diagnosis is aimed solely at proper clinical management by focusing attention toward interventions directed at the underlying medical condition or the physiologic effects of the drugs driving the symptoms. The fact that the etiology of OCD can include particular medical conditions or drugs should provide evidence for an analysis of the neurobiology and plausible pathophysiology of OCD (Szechtman et al., 2014). Indeed, later we review a wide range of empirical findings about such factors, as they may provide clues to illuminate the underlying neurobiology of the disorder.

\section{B. Pharmacotherapy for Obsessive-Compulsive Disorder in Clinical Practice}

1. Monotherapy with Serotonin Reuptake Inhibitors. One of the seminal serendipitous discoveries in psychiatry was the incidental observation by Spanish psychiatrists (Fernández Córdoba and López-Ibor Aliño, 1967) that the tricyclic antidepressant (TCA), clomipramine, provided relief also for "obsessional" symptoms in patients with depression (Yaryura-Tobias and Neziroglu, 1975; Philpott, 1976; Ananth, 1986). This clinical impression contradicted the hitherto widely held belief that OCD is refractory to pharmacotherapy (Goodman et al., 2014), sparking extensive and fruitful investigations of pharmacotherapy not only for OCD comorbid with depression but also for OCD in patients without this comorbid psychiatric diagnosis. Repeatedly, by 1990, many studies confirmed the effectiveness of clomipramine in OCD. For instance, a large multicenter double-blind clinical trial with $>500$ patients showed a reduction in YBOCS scores of approximately $40 \%$ after 10 weeks of treatment compared with only about a $4 \%$ reduction for the placebo-treated patients (Clomipramine Collaborative Study Group, 1991).

Clomipramine, like other TCAs, is an inhibitor of serotonin and norepinephrine reuptake; however, as a tertiary amine TCA, clomipramine is a more potent inhibitor of serotonin reuptake than secondary amine TCAs such as nortriptyline and desipramine (Wishart et al., 2018; https://www.drugbank.ca/drugs/DB01242). TCAs without potent effects on serotonin reuptake show little efficacy on OCD (Piccinelli et al., 1995), raising the hypothesis that blockade of serotonin reuptake is essential for a therapeutic effect in OCD. Support for this hypothesis came with the introduction of selective serotonin reuptake inhibitors (SSRIs) that have little or no effect on norepinephrine and dopamine reuptake, despite distinct structural differences between compounds in this class (Wishart et al., 2018). As predicted, SSRIs proved clinically effective, with response rates of $40 \%-60 \%$ versus $<20 \%$ with placebo (Soomro et al., 2008; Katzman et al., 2014); a clinical response was defined as a $\geq 25 \%$ reduction in symptoms (Soomro et al., 2008) measured with the YBOCS (Goodman et al., 1989b; Kim et al., 1990). SSRIs are now the clinically recommended first line of pharmacotherapy for OCD (Koran and Simpson, 2013; Katzman et al., 2014; Pittenger and Bloch, 2014; Fineberg et al., 2015; Skapinakis et al., 2016a). The first-line SSRI compounds for OCD are listed in Table 2 and include the following drugs: fluvoxamine (Goodman et al., 1990), fluoxetine (López-Ibor et al., 1996), paroxetine (Zohar and Judge, 1996), sertraline (Hoehn-Saric et al., 2000), and escitalopram (Stein et al., 2007).

Surprisingly, SSRIs proved no better than clomipramine for OCD and possibly even somewhat less efficacious. In a comprehensive network meta-analysis comparing pharmacological interventions for OCD in 
TABLE 2

Recommendation for pharmacotherapy for OCD

\begin{tabular}{|c|c|}
\hline & Treatment \\
\hline First line & $\begin{array}{l}\text { Escitalopram, fluoxetine, fluvoxamine, } \\
\text { paroxetine, sertraline }\end{array}$ \\
\hline Second line & $\begin{array}{l}\text { Citalopram, clomipramine, mirtazapine, } \\
\text { venlafaxine XR }\end{array}$ \\
\hline Third line & $\begin{array}{l}\text { IV citalopram, IV clomipramine, duloxetine, } \\
\text { phenelzine, tramadol, tranylcypromine }\end{array}$ \\
\hline \multirow[t]{4}{*}{ Adjunctive therapy } & First line: aripiprazole, risperidone \\
\hline & Second line: memantine, quetiapine, topiramate \\
\hline & $\begin{array}{l}\text { Third line: amisulpride, celecoxib, citalopram, } \\
\text { granisetron, haloperidol, IV ketamine, } \\
\text { mirtazapine, N-acetylcysteine, olanzapine, } \\
\text { ondansetron, pindolol, pregabalin, riluzole, } \\
\text { ziprasidone }\end{array}$ \\
\hline & $\begin{array}{l}\text { Not recommended: buspirone, clonazepam, } \\
\text { lithium, morphine }\end{array}$ \\
\hline Not recommended & Clonazepam, clonidine, desipramine \\
\hline
\end{tabular}

IV, intravenous; XR, extended release. Reprinted from "Canadian clinical practice guidelines for the management of anxiety, posttraumatic stress and obsessivecompulsive disorders," by Katzman et al. (2014), BMC Psychiatry, 14 Suppl 1(1), S1. CC BY 4.0 .

over 3000 patients, SSRIs produced a poorer reduction in YBOCS score than clomipramine, but this trend was not statistically significant (Skapinakis et al., 2016a). However, the side effects of SSRIs were less bothersome, making treatment with SSRIs more acceptable to patients than treatment with clomipramine (Skapinakis et al., 2016a). Because of poorer acceptability than SSRIs, clomipramine is relegated as a second line of pharmacotherapy treatment of OCD (Katzman et al., 2014), as noted in Table 2.

Interestingly, in the foregoing network meta-analysis (composed of studies until 2016), the mean reduction in YBOCS score over drug placebo was relatively modest for SSRI drugs as a whole (class effect, -3.49 [95\% credible interval, -5.12 to -1.81 ]; 37 trials and 3158 patients), with very little difference between the different SSRIs (Skapinakis et al., 2016b). These findings are consistent with a prior meta-analysis (Soomro et al., 2008). The small average reduction in YBOCS score is consistent with the finding noted earlier that although many patients do achieve clinical relief with SRI pharmacotherapy, equally many $(40 \%-60 \%)$ do not find clinical relief for their OCD (Soomro et al., 2008; Katzman et al., 2014).

What do the pharmacotherapy findings reveal about OCD? As previously summarized, the serendipitous discovery that clomipramine could benefit OCD led to concerted research effort establishing treatment parameters of pharmacotherapy for OCD. Moreover, findings from treatment-response studies were used to generate hypotheses as to the etiology of OCD (Goodman et al., 2014), an early one being that OCD reflects a dysfunction in the brain serotonergic system (Yaryura-Tobias et al., 1976). This hypothesis received support from the clinical evidence that antidepressants targeting serotonin reuptake were more efficacious for OCD than agents with minor effects on serotonin reuptake (Leonard et al., 1989; Goodman et al., 1990; Piccinelli et al., 1995; Hoehn-Saric et al., 2000). However, this approach-inferring etiology and pathophysiology from treatment response-is "fraught with problems that have plagued the field of psychiatry for decades ... [and] has yielded few new insights or novel treatments" (Goodman et al., 2014, p. 261). Although psychiatry is well equipped to evaluate treatments to best manage mental disorders (Berrios and Marková, 2002), the deduction of etiology from findings of treatment effect is a logical fallacy, the "fallacy of confirming the consequence" (Montgomery, 2012). As we elaborate later, etiology and pathophysiology require research using the tools of a basic science discipline to experimentally manipulate the putative causes (Szechtman and Eilam, 2005; Bolkan et al., 2016).

Nevertheless, findings of treatment effect with drugs are very useful and important, not only because they inform the clinical management of the disorder but also because they establish factors with effects on OCD. Any good theory of OCD must incorporate and account for such empirically established factors and facts. However, drug treatment effects, rather than being regarded as evidence or proofs of etiology, are best viewed more broadly, as suggesting hypotheses about mechanisms by which a disordered system is returned to normal function. As we elaborate in section V, such hypotheses must consider more than just the obvious possibility that the drug treatment corrects the etiological fault yielding the disorder. It is also plausible that the therapeutic effect is exerted by an action not at the site of the neurobiological fault but elsewhere, by enhancing systems supporting the operation of the circuit yielding OCD.

Aside from targeting serotonin reuptake in OCD, pharmacotherapy treatment studies established three attributes of SSRI intervention that need to be considered in a theory of OCD. First, OCD pharmacotherapy does not result in an immediate effect on symptom reduction, unlike for instance the effects of morphine on pain reduction (Dubuisson and Dennis, 1977). Instead, it takes weeks of treatment with SSRIs for clinical improvement to be evident; practice guidelines recommend a trial of at least 10-12 weeks (Koran et al., 2007). This apparent delay may suggest that improvement in clinical symptoms follows a step function but a recent meta-analysis of time to response with SSRIs shows otherwise (Issari et al., 2016). The researchers identified randomized, placebo-controlled SSRI trials in OCD published until 2013 and extracted the YBOCS score as a function of weeks of SSRI treatment (up to 12 weeks of treatment). The best model that fitted these data was a logarithmic response curve. That is, the index of OCD symptoms (YBOCS score) declined as a function of the logarithm of the duration of SSRI treatment (Issari et al., 2016); a similar logarithmic response curve was observed with pharmacotherapy in pediatric OCD (Varigonda et al., 2016). 
Being a logarithmic function, the data indicate that the highest incremental gain from treatment occurs during the first 2 weeks of exposure to SSRIs and conversely that gains in symptom improvement decline with continued exposure to SSRIs (Issari et al., 2016). In other words, although clinical relief is evident after 1012 weeks of treatment, it does not materialize at that time point all at once. One interpretation of this finding is that with pharmacotherapy there is buildup over time of a cascade of processes that ultimately restore a normal phenotype and/or compensate for the dysfunction manifested as OCD. Interestingly, preclinical research has shown the phenomenon of "time-dependent sensitization" (Antelman and Chiodo, 1981; Antelman et al., 2000; Harvey et al., 2003), in which the chronic effects of a drug are equivalent to a single injection of the drug - as long as the passage of time is the same in the two conditions; that is, the number of days after acute treatment is the same as the duration of chronic treatment (Antelman et al., 2000). This suggests that the final effect does not depend on a continuous administration of the drug but rather on the first few injections and the passage of time for the effect to develop fully. Hence, the logarithmic response curve in OCD provides a rationale to determine whether continuous drug intake for 10-12 weeks is necessary or whether 1 to 2 weeks of pharmacotherapy and the passage of time are sufficient for the clinical benefits to be evident at the 10- to 12 -week time mark.

Second, the antidepressant drugs used in the treatment of OCD are not specifically for OCD; they are used as first-line agents in the treatment of a range of psychiatric disorders, including depression, panic disorder, social anxiety, generalized anxiety disorder, and post-traumatic stress disorder (PTSD) (Katzman et al., 2014). This suggests that the serotonergic reuptake targeted by OCD pharmacotherapy belongs to more than one functional network.

Finally, there is a high percentage of patients with OCD who do not benefit from intervention with SRIs, regardless of agent, dose, or duration of treatment (Katzman et al., 2014; Pittenger and Bloch, 2014). A common interpretation of this finding is the hypothesis that OCD symptoms are mediated by more than one neurochemical system. Clinically, the inadequacy of monotherapy has spurred clinicians to combine another agent with an SSRI, an approach known as "adjunctive" or "augmentation" therapy and which we consider next.

2. Augmentation of Selective Serotonin Reuptake Inhibitor Pharmacotherapy with Antipsychotic Medication. Over the decades, clinicians have sampled a range of potential compounds to increase the therapeutic effectiveness of SSRIs (Hollander et al., 2000; Denys, 2006; Katzman et al., 2014), but the evidence for clinical efficacy based on meta-analysis of randomized trials is available thus far only for neuroleptics (Bloch et al., 2006; Skapinakis et al., 2007; Dold et al., 2013, 2015; Veale et al., 2014). One early open trial using neuroleptics to supplement treatment with SSRIs in OCD was published in 1990 (McDougle et al., 1990). In an attempt to improve the clinical outcome of 17 patients who were "unresponsive to a placebocontrolled trial of fluvoxamine with or without lithium," the clinicians added pimozide (14 patients), thioridazine (two patients), or thiothixene (one patient) to the patients' ongoing treatment regimen for 2-8 weeks. The addition of a neuroleptic produced a significant mean \pm S.D. decline of $9.1 \pm 8.9$ points in the YBOCS score in this sample of patients compared with the score before addition of neuroleptics. Moreover, nine of the 17 patients met the criterion of a "responder" on the basis of being "much improved" or "very much improved" on the Clinical Global Impression scale; there was a $62 \%$ decline in YBOCS score for these patients. Considering these clinical findings, the authors raised the hypothesis that "both the serotonin and dopamine systems may be involved in the mediation of obsessive-compulsive symptoms" (McDougle et al., 1990, p. 654).

Bloch et al. (2006) published the first meta-analysis of double-blind, randomized controlled clinical trials evaluating antipsychotic agents as an augmentation strategy in adults with treatment-refractory OCD; "treatment-refractory" was defined by the lack of response to at least 2 months of traditional treatment with SSRIs. For the meta-analysis, nine trials met the inclusion criteria, with a total of 278 patients with OCD (143 receiving antipsychotic augmentation and 135 receiving placebo augmentation). The antipsychotics used for augmentation in those trials were olanzapine (two trials), quetiapine (three trials), risperidone (three trials), and haloperidol (one trial). There was a statistically significant benefit from neuroleptic augmentation, with a response rate of $32 \%$ in the antipsychotic augmentation group (46 of 143 patients with OCD) compared with $11 \%$ in the placebo group (15 of 135 patients with OCD). Response to treatment was defined as a reduction $>35 \%$ in YBOCS rating from baseline.

Subsequent meta-analyses that included additional trials confirmed the efficacy of neuroleptic augmentation in treatment-resistant OCD (Skapinakis et al., 2007; Dold et al., 2013, 2015; Veale et al., 2014). The latest meta-analysis (Dold et al., 2015) included all studies meeting criteria published from 1994 to 2013: 14 double-blind, randomized, placebo-controlled trials for a total of 491 patients with OCD, investigating the antipsychotics quetiapine (four trials, 142 patients), risperidone (four trials, 132 patients), aripiprazole (two trials, 79 patients), olanzapine (two trials, 70 patients), paliperidone (one trial, 34 patients), and haloperidol (one trial, 34 patients). This meta-analysis confirmed that augmentation with antipsychotics was significantly 
more efficacious than placebo in YBOCS score reduction, with the difference between the YBOCS total reduction in the pooled antipsychotic group and the pooled placebo group being 4.02 points. Moreover, augmentation with aripiprazole, haloperidol, or risperidone was significantly superior over augmentation with placebo, but augmentation with olanzapine, paliperidone, or quetiapine was not. Finally, in terms of clinical response rate $(\geq 35 \%$ YBOCS improvement), there was a significant advantage for adjunctive treatment with antipsychotics $(29.8 \%$ of patients improved) compared with the response rate with placebo augmentation (12.5\% of patients improved). In other words, one of three patients with treatmentresistant OCD benefited from antipsychotic adjunctive therapy compared with one of eight patients who benefited from adjunctive therapy with a placebo. In all, the authors concluded that their meta-analysis shows that "augmentation treatment with antipsychotics can be regarded as evidence-based treatment strategy in OCD patients refractory to monotherapy with SRIs" (Dold et al., 2015).

As the foregoing summary makes plain, even though augmentation with neuroleptics can be of clinical benefit, this adjunctive therapy is twice as likely to be without clinical benefit. Indeed, in a recent report that assessed consecutive OCD outpatients in nine international centers to evaluate how nonresponders to first-line treatment are managed in clinical practice, it was found that $50 \%$ of the patients were receiving augmentation therapy (Van Ameringen et al., 2014). However, consistent with the meta-analysis that the likelihood of clinical improvement from antipsychotic augmentation is relatively low, the authors noted that "despite evidence from academic clinical trials that pharmacological augmentation may be effective in OCD, analysis from these real-life settings suggest[s] that this treatment strategy may be less valuable" (Van Ameringen et al., 2014, p. 600).

Nevertheless, even though the necessary conditions have not been identified, the empirical evidence from some patients of a positive therapeutic effect with neuroleptic augmentation establishes this drug effect as a factor with potential impact on OCD. Considering that neuroleptics block dopamine receptors (Seeman, 1980), the activity of dopamine systems may play a role in the expression of the OCD phenotype. To investigate such a hypothesis, a basic science research approach in which putative causes can be manipulated experimentally is important. Interestingly, preclinical research has shown that chronic treatment with the dopamine agonist, quinpirole, induces behavioral features of compulsions in rats that are also shown by patients with OCD (Szechtman et al., 1998). As such, this preparation provides an experimental paradigm to investigate a potential role of dopamine activity in OCD.
3. Augmentation of Selective Serotonin Reuptake Inhibitor Pharmacotherapy with Psychologic Treatment. In addition to first-line pharmacotherapy for OCD (Table 2), there is also a nonpharmacological intervention that is recommended as a first-line therapy option for OCD: namely, CBT, generally in the form of ERP (Koran and Simpson, 2013; Katzman et al., 2014; Seibell et al., 2017). The various forms and aspects of psychologic therapy will be described later in section II.D, but here we consider its use with SRI pharmacotherapy as an augmentation strategy.

Confronted with the evidence that antipsychotic augmentation may be of benefit to only one in three patients receiving SSRI monotherapy (Dold et al., 2015), clinicians examined whether pharmacotherapy combined with psychologic therapy could provide greater relief to patients with OCD (Greist, 1992). It turns out that this question is not simple to examine empirically. In clinical practice, treatment entails not just pill dispensing but also personal interactions between the patient and health care provider that could constitute a form of psychologic intervention, although clearly not a structured technique such as ERP. Indeed, when evaluating a pharmacotherapy regimen, investigators may acknowledge such a possibility by stating, for example: "Formal behavior therapy was not given" (McDougle et al., 1990, p. 653). Nevertheless, there are several published empirical investigations evaluating CBT augmentation.

Foa et al. (2005) compared four groups of patients with $\operatorname{OCD}(N=122$ entrants $)$ in a double-blind, randomized, placebo-controlled trial. One group of patients received ERP therapy, a second group received clomipramine pharmacotherapy, a third group received both treatments (ERP and clomipramine), and the control group received a pill placebo. Outcome measures included YBOCS score and ratings on the Clinical Global Impression scales, where a score of "much improved" or "very much improved" was used to define the presence of a "clinical response" (Foa et al., 2005). At the end of the 12-week treatment period, YBOCS scores declined from $25.0 \pm 4.0$ to $22.2 \pm 6.4$ for the placebo control group, from $26.3 \pm 4.4$ to $18.2 \pm 7.8$ for the clomipramine monotherapy group, from $24.6 \pm 4.8$ to $11.0 \pm 7.9$ for the ERP monotherapy group, and from $25.4 \pm 4.6$ to $10.5 \pm 8.2$ for the clomipramine augmented with ERP group, with each active treatment regimen being statistically superior to placebo. Moreover, the YBOCS scores for the ERP monotherapy and the clomipramine augmented with ERP groups were significantly different from the clomipramine monotherapy group, but not from each other. Thus, although augmentation with ERP is superior to clomipramine monotherapy, such adjunctive therapy is clinically no better than mere ERP monotherapy by itself. The "clinical response" data showed a similar profile of results, with a response rate of $8 \%$ for patients treated with pill placebo, $42 \%$ for 
those treated with clomipramine monotherapy, $62 \%$ for patients treated with ERP monotherapy, and $70 \%$ for patients treated with clomipramine augmented with ERP (Foa et al., 2005).

It is of theoretical importance to unravel why ERP augmentation of clomipramine treatment was clinically equivalent to ERP monotherapy (Foa et al., 2005), as this would reveal whether the clomipramine and the ERP treatments exert their effects on the same or relatively independent mechanisms underlying OCD. Unfortunately, the study design did not permit a resolution. Nevertheless, it is noteworthy that the profiles in the reduction of YBOCS scores had a different time course with clomipramine monotherapy than with ERP monotherapy. Inspection of the graphs for YBOCS ratings at the 0-, 4-, 8-, and 12-week assessment points (see Fig. 2 in Foa et al., 2005) shows that the decline in symptoms in the clomipramine monotherapy group followed the typical curve described earlier for SSRIs (Issari et al., 2016); however, the decline in symptoms in the ERP monotherapy group appeared to have reached an asymptote at the 4 -week assessment point. The profile of the clomipramine plus ERP group was identical to the ERP monotherapy group. The distinct profiles suggest that although symptom improvement with drug monotherapy is incremental over a 12 -week period, the rate of improvement is much more rapid with ERP and all YBOCS score reduction occurs in the first 4 weeks of treatment. The fact that clomipramine and ERP therapies did not produce an additive effect in 12 weeks of treatment suggests to us that both treatments exert their effects on the same mechanism underlying OCD.

The time course of improvement with ERP therapy appears temporally aligned to the schedule and intensity of ERP treatment, which involved a 2-hour session (each weekday) in the first 3 weeks of treatment, two visits by the therapist to the patient's home ( 4 hours total) in the fourth week of treatment, and a 45-minute session with the therapist at weekly intervals in the remaining 8 weeks of treatment (Foa et al., 2005). Thus, the gains on YBOCS score reduction with ERP therapy all occurred within the 4 weeks of sustained interaction with the therapist. By contrast, patients without ERP therapy met with a therapist only for 30 minutes at weekly intervals and the size of their incremental gains was modest but evident throughout the 12 weeks of treatment. One hypothesis suggested by these timecourse data is that massed application may yield clinical benefit sooner than temporally spaced application of such interventions.

The possibility that a massed versus spaced administration of treatments may impact the therapeutic effect is enhanced by findings from a later study examining the effectiveness of ERP augmentation in patients who were refractory to SRI pharmacotherapy (Simpson et al., 2008). Patients entering this study had an adequate trial of SRI pharmacotherapy for at least
12 weeks but with little clinical relief. Patients continued with their medication, but half $(n=56)$ were randomly assigned to receive ERP augmentation over an 8-week trial period and the other half $(n=55)$ received an equivalent amount of stress-management training. For both ERP and stress-management training, there were 17 twice-weekly sessions with a therapist (each 90-120 minutes), daily homework assignments, and between-session phone calls (twice per week, each $<20$ minutes). Independent evaluators blind to treatment rated OCD symptom severity with YBOCS at weeks 0,4 , and 8 .

By the end of treatment, the YBOCS score declined from $25.4 \pm 4.7$ to $14.2 \pm 6.6$ in patients with ERP augmentation, but only from $26.2 \pm 4.4$ to $22.6 \pm 6.3$ in the group with stress-management augmentation. Thus, this study formally affirmed that ERP augmentation is effective also in patients who have not benefited from SRI monotherapy. However, the study also illuminated the dynamics of YBOCS score reduction during the course of treatment. Inspection of the profile of YBOCS improvement (Fig. 2 in Simpson et al., 2008) shows relatively steady gains during the entire course of the 8-week trial, in contrast to the study of Foa et al. (2005) in which all of the improvement with ERP occurred in the first 4 weeks of treatment. Of importance, the number of ERP treatment sessions was equivalent in the two studies (17 sessions) but they were delivered over the course of 4 weeks in the Foa et al. (2005) study and over the course of 8 weeks in the Simpson et al. (2008) study. Thus, the massed administration of ERP in the Foa et al. (2005) study produced a rapid (within 4 weeks) decline in YBOCS score, whereas the spaced administration of the same number of ERP interventions in the Simpson et al. (2008) study produced a gradual decline in YBOCS score over the 8 weeks of treatment. Thus, comparison of the two clinical trials strongly suggests that the mechanism underlying OCD is more attuned to massed than to spaced psychologic interventions.

The foregoing studies provide the strongest evidence that ERP augmentation is superior to SRI monotherapy, but not all studies show such large effects. Nevertheless, a comprehensive meta-analysis of available studies supported the conclusion that "the combination of SSRIs or clomipramine with psychotherapy is likely to offer more benefit to patients with severe illness than is monotherapy, but more research is needed than at present to support this hypothesis" (Skapinakis et al., 2016b, p. 736). Moreover, Skapinakis et al., 2016b note that "the question of what is better as monotherapy in obsessive-compulsive disorder-medications or psychotherapy-cannot be answered given the current evidence" (p. 736). The empirical evidence is inadequate because most psychotherapy trials allowed the inclusion of patients who were also taking antidepressant medication (Skapinakis, 2016; Skapinakis et al., 2016a). 
However, regardless of whether any form of therapy is more efficacious overall, clinicians do not have a theoretically grounded rationale for which treatment best fits the conditions of specific patients. This may explain in part why a significant portion of patients with OCD do not achieve relief and remission in clinical practice (Tolin et al., 2005; Simpson et al., 2006; Farris et al., 2013; Storch et al., 2015; Mataix-Cols et al., 2016).

\section{Neuromodulatory Therapies for Obsessive- Compulsive Disorder}

1. Psychosurgery. The pharmacotherapy for OCD reviewed earlier is directed at chemical neurotransmission, but an older medical intervention for psychiatric disorders is "psychosurgery," which targets the anatomy of the brain itself (for reviews on the use of psychosurgery to treat OCD and other psychiatric disorders, see Leiphart and Valone, 2010; Patel et al., 2013; Dyster et al., 2016; Neumaier et al., 2017). Stereotaxic psychosurgery is indicated today for refractory OCD and treatment-resistant depression (Bari et al., 2018). Good therapeutic success for OCD has been obtained with two surgical procedures: capsulotomy and cingulotomy. Capsulotomy targets the anterior limb of the internal capsule (ALIC) region and cingulotomy targets the cingulate bundle region (Neumaier et al., 2017).

Where did the targets for OCD psychosurgery come from? As noted in a recent review, "modern ablative procedures for OCD were largely developed empirically but all evolved from increasingly selective disconnections of limbic fibers or frontal white matter tracts leading to the limbic system" (Tierney et al., 2014, p. 395). As analyzed by Berrios (1997), the scientific rationale for interrupting frontal lobe projections was articulated by Egas Moniz, who pioneered the leucotomy surgical approach in the 1930s and received the Nobel Prize in Medicine in 1949 "for his discovery of the therapeutic value of leucotomy in certain psychoses" (https://www.nobelprize.org/nobel_prizes/medicine/ laureates/). Consistent with the prevalent conceptualization that "mental faculties were sited in specific brain regions" and that mental illness "results from multiple lesions (whether anatomical or physiological)," Moniz believed that there were "aberrant, perseverative, facilitated circuits responsible for [certain troublesome] mental (emotional) symptoms, and that these were sited in the pre-frontal cortex. Consequently, he was led to deduce that their partial damage would abolish the relevant symptoms" (Berrios, 1997, p. 77). Interestingly, the rationale for modern psychosurgery is based on similar belief that a limited controlled lesion targeting some particular prefrontal connection will yield a reduction in the expression of certain symptoms (Berrios, 1997).

A recent report (Lopes et al., 2014) examined the effectiveness of capsulotomy for refractory OCD in a double-blind, placebo-controlled, randomized clinical trial. Eight patients received ablative radiosurgery at the ventral border of the ALIC and eight patients received sham radiosurgery using the same equipment; blinding was unmasked at 12 months postsurgery and the sham group was offered active surgery. Evaluation at the 1-year time point showed that two of the eight surgery patients and none of the sham surgery controls responded to the treatment (defined as a decline in YBOCS severity of at least 35\%). Three additional patients showed the full response at 54 months postsurgery; moreover, two of the four sham surgery patients who later received capsulotomy showed the full response at 12 months postsurgery. This clinical trial provides encouraging evidence for the effectiveness of capsulotomy over sham surgery, and the results suggest that this beneficial outcome may take longer than 1 year to be manifested.

There is currently no comparable clinical trial for the effectiveness of cingulotomy. However, a systematic review of the literature considers the available open-label psychosurgery studies for refractory OCD and compares the effectiveness of cingulotomy with that of capsulotomy (Brown et al., 2016). On the basis of the same criterion for full recovery (a decline in YBOCS severity of at least $35 \%$ ), there was a comparable response rate for the two procedures at the last follow-up: $41 \%$ at 47 months for cingulotomy and $54 \%$ at 60 months for capsulotomy.

From its very beginnings, the theoretical and ethical rationale for the psychosurgical approach has been controversial and counterintuitive. As one skeptical psychiatrist opined in 1937, "Mutilation of an organ "had never led to an improvement of its function" (quoted in Berrios, 1997, p. 78). Yet regardless of how counterintuitive it appears, case reports do exist of remission of OCD symptoms after brain damage. In a review of the literature, Figee et al. (2013) identified six individuals who had longstanding OCD but in whom these symptoms resolved after acquiring a lesion (due to infarction or hemorrhage) in the "left putamen, left internal capsule genu, left frontal lobe and right posterior frontoparietal cortex" (p. 335).

The complexity of understanding the mechanisms underlying therapeutic effects of psychosurgery is underscored in the same review by case reports of individuals who acquired OCD symptoms after suffering brain damage due to infarction or hemorrhage. One study identified reports of 71 individuals who acquired various symptoms characteristic of OCD after suffering a lesion in various brain areas, including regions targeted by psychosurgery such as the basal ganglia, caudate nucleus/nucleus accumbens, putamen, globus pallidus, internal capsule, thalamus, limbic system, frontal cortex, parietal lobe, temporal lobe, cerebellum, brainstem, hypothalamus, or pineal area (Figee et al., 2013). Clearly, mere identity of neuroanatomical location is not sufficient to understand OCD and its treatment, since a lesion in the same region can both ameliorate and induce OCD symptoms. 
2. Deep Brain Stimulation. Like the psychosurgery intervention for psychiatric disorders, DBS is another medical therapy that targets the anatomy of the brain. After the success of DBS in replacing stereotaxic ablation as a treatment modality for movement disorders (Benabid et al., 1998; Benabid, 2003), DBS is currently the preferred treatment modality over psychosurgery for refractory psychiatric disorders. The choice of DBS stems largely from practical and ethical considerations: unlike a lesion, DBS intervention does not have the appearance of producing irreversible damage to brain tissue, and the parameters of the stimulation can be adjusted and fine-tuned postsurgery for a "best" effect on the patient's symptoms (Bari et al., 2018). The first publication of DBS for refractory OCD reported positive effects in three of the four patients who had electrodes implanted bilaterally for focal stimulation of the sites normally targeted by capsulotomy (i.e., the ALIC region that connects the frontal lobe and thalamus; Nuttin et al., 1999). The reported therapeutic promise instigated many studies, with investigators refining stimulation parameters and targets for a therapeutic effect.

Four targets received the most concerted study. One site is referred to as "VC/VS" and is a region at the junction of the ventral ALIC and the adjacent ventral striatum (Greenberg et al., 2010). Results of a multicenter open-label trial showed symptomatic improvement in approximately two-thirds of patients with refractory OCD (Greenberg et al., 2010). A randomized, doubleblind, sham-controlled clinical trial led by the same investigators is expected to be completed in August 2020 (ClinicalTrials.gov NCT00640133).

Another site where DBS for refractory OCD showed positive effects is the bed nucleus of the stria terminalis (BST). In a study with 24 patients with refractory OCD, electrode placements into the ALIC region included stimulation of the BST; the authors refer to these placements as "stimulation in the ALIC/BST area" (Luyten et al., 2016). There were significant reductions in YBOCS scores not only during the double-blind, randomized crossover trial (where the scores are compared during periods when DBS is turned on vs. when it is turned off) but also after 4 years of follow-up. Since many of the patients had trials of focal stimulation in the ALIC and BST, the investigators sought to determine whether one of these sites was therapeutically superior. Indeed, there were indications that BST was a "better target" on several measures, including the rate of responders at last follow-up (83\% for patients with BST stimulation vs. $67 \%$ for the entire group of patients). Nevertheless, Luyten et al. (2016) remained puzzled as to "why DBS in BST (and ALIC) seems to have extraordinary results in some patients, yet unsatisfactory effects in others" (p. 1279).

Yet another DBS target in the ALIC region is the nucleus accumbens (Denys et al., 2010). Of the 16 patients with refractory OCD who received 8 months of open-label DBS stimulation to the nucleus accumbens, nine (56\%) were responders (a decrease of YBOCS $\geq 35 \%$ from presurgery). This open-label phase was followed by a double-blind crossover trial with 2 weeks of stimulation and 2 weeks of sham stimulation $(n=14)$, showing a significant (25\%) reduction in YBOCS score between sham and active stimulation. The beneficial effects of DBS were also evident at the end of an open 12-month maintenance phase, indicating that bilateral DBS of the nucleus accumbens "may be an effective and safe treatment for treatment-refractory OCD" (Denys et al., 2010, p. 1061).

The last DBS target lies outside the region historically targeted by psychosurgery and is the subthalamic nucleus (STN). This target was suggested by incidental findings in two patients with Parkinson disease (PD) treated with DBS to the STN. As expected, their parkinsonian disability improved but so did their comorbid OCD symptoms, reducing YBOCS scores by $58 \%$ and $64 \%$ (Mallet et al., 2002). Those serendipitous clinical observations instigated a multicenter, randomized, double-blind, crossover study comparing stimulation of the STN with sham stimulation in 16 patients with refractory OCD (Mallet et al., 2008). Eight randomly assigned patients underwent 3 months of DBS and 3 months of sham DBS stimulation 1 month later. The other eight patients received those treatments in the reverse order. There was a $32 \%$ reduction in YBOCS scores with active DBS stimulation compared with sham DBS, leading the researchers to suggest that "stimulation of the subthalamic nucleus may reduce the symptoms of severe forms of OCD" (Mallet et al., 2008, p. 2121).

Although two meta-analyses of DBS in refractory OCD are available (Hamani et al., 2014; Kisely et al., 2014), there are insufficient data to compare which of the DBS targets is more effective. However, a recent review of the literature compared published reports using anterior capsulotomy versus DBS targeting this area (VS/VS and nucleus accumbens) for OCD (Pepper et al., 2015). Both procedures were found to be effective with somewhat better success for psychosurgery, leading to the conclusion that "the current popularity of DBS over ablative surgery for OCD is not due to non-efficacy of AC [anterior capsulotomy], but possibly because DBS is perceived as more acceptable by clinicians and patients" (Pepper et al., 2015, p. 1028).

Most reports for effectiveness of DBS in OCD include an open-label phase, raising concerns that "attempting to control for expectation of benefit in efficacy outcomes in DBS is extremely challenging" (Mestre et al., 2016, p. 294). It is instructive that a very recent workshop grappled with this issue in the context of depression, another psychiatric disorder for which there are many open-label studies showing the therapeutic effectiveness of DBS. Yet two large double-blind studies for DBS in depression had to be aborted upon an interim 
analysis due to the likelihood that DBS superiority would not be evident (Bari et al., 2018). The workshop considered a number of possible reasons for the observed failure, ranging from choice of brain sites and DBS stimulation parameters to suitable paradigms for the statistical evaluation of clinical trials for psychiatric disorders. However, one should also question whether it is even theoretically feasible to have a therapeutic effect for a psychiatric disorder such as OCD using a "doubleblind" paradigm, where by virtue of study design the patient remains distrustful of help but the psychologic meaning of treatment is crucial to the clinical effect as discussed later (see section II.D).

What are the mechanisms for the therapeutic effect of DBS in OCD? Considering that focal stimulation was applied to the same region as targeted by ablative psychosurgery (Pepper et al., 2015), the earliest notion viewed DBS as creating a "functional lesion" by inhibiting the stimulated target region, akin to the therapeutic mechanism first suggested in PD (Benabid, 2003). However, the notion of a "functional lesion" is grossly inadequate for PD (Montgomery and Gale, 2008) and hence probably for OCD as well.

In their review, Montgomery and Gale (2008) described the evidence against the functional lesion hypothesis of DBS in PD, elaborated on the complexity of physiologic effects produced by DBS stimulation, highlighted a relatively novel "systems oscillators theory" to conceptualize the normal electroneurophysiology of basal ganglia circuits, and considered hypotheses regarding DBS mode of action within the framework of normal basal ganglia function. Of particular relevance for the purposes of this review are three notions: that the effects of DBS are best viewed from a systems perspective rather than considering "just its local effects"; that the anatomic architecture of the basal ganglia-thalamus-cortex (BG-TH-CTX) connections (Parent and Hazrati, 1995a,b) should be considered as a network of a large set of nested nonlinear reentrant, loosely coupled oscillators capable of spontaneous or stimulus initiated self-organization; and that DBS at appropriate parameters introduces into these loops a suitable oscillator that provides "stochastic resonance" enhancing the proper flow of information through the network (Montgomery, 2007, 2016, 2017; Montgomery and Gale, 2008; Montgomery and He, 2016).

This conceptualization contrasts with our opening statement that DBS "is another medical therapy that targets the anatomy of the brain." According to the foregoing notions, the target of DBS is not a specific neuroanatomical location as such. Rather, the target is information flow through the BG-TH-CTX loops and DBS is a procedure to restore the functional integrity of the information being transmitted. The nervous system transmits information electronically; hence, the flow of informational content through the circuits can be modulated through the interjection of an appropriate pattern of electrical pulses into a loop, as delivered with DBS. As such, the crucial variable is not the specific anatomic target of DBS; rather, the crucial variable is a convenient location for DBS to interject electrical signals into the appropriate loops, and to do so without impinging on the operation of unrelated circuits because their stimulation may result in undesirable "side effects." Indeed, the STN is one such convenient location because the interjection there of a DBS oscillator accesses several relevant BG-THCTX loops (Montgomery, 2007; McIntyre and Hahn, 2010).

Nevertheless, preclinical studies with animal models of OCD suggest that the selection of neuroanatomical site for DBS stimulation extends beyond its convenient location-its choice may bear also on DBS therapeutic effect. Using two different animal models of OCD in parallel, including the signal attenuation (SA) (Joel, 2006) and the quinpirole sensitization (QS) (Szechtman et al., 1998) rat models of OCD, Winter et al. found that DBS in STN did indeed reduce measures of compulsive behavior in both models (Winter et al., 2008; Klavir et al., 2009). However, when the target was the globus pallidus, DBS was fully effective only in one model, the SA model (Djodari-Irani et al., 2011; Klavir et al., 2011). Hence, the neuroanatomical site can impact the effectiveness of DBS, related perhaps to a different aspect of the neurobiology of OCD captured by each model (Szechtman et al., 2017).

Indeed, it would be expected that neuroanatomical sites in the BG-TH-CTX network are not all equivalent, since OCD is likely related to only some of the many oscillator loops in the BG-TH-CTX network and "nodes in different structures are common to different sets of oscillators" (Montgomery, 2007, p. 460). In other words, not all nodes are in every neuroanatomical structure and to be effective in OCD, DBS must target the spatially distributed set of pertinent nodes.

3. Noninvasive Brain Stimulation. Similar to DBS, two other types of neurostimulation have been investigated as a possible intervention for OCD: transcranial magnetic stimulation (TMS; Lefaucheur et al., 2014) and tDCS (Lefaucheur et al., 2017).

a. Repetitive Transcranial Magnetic Stimulation. In contrast to DBS, which stimulates brain tissue through an implanted electrode, TMS stimulates the brain with a noninvasive device through electromagnetic induction (Hallett, 2007; Lefaucheur et al., 2014; Rossini et al., 2015). The device is placed on the head and is programmed to generate brief magnetic pulses; those pulses in turn induce an electric field in the underlying brain tissue. The resulting electric current has both local and network effects: a local effect by stimulating neurophysiological changes at the site of stimulation, and a network effect by virtue of stimulating the local nodes of neural circuits and so activating neuroanatomical networks incorporating the target 
region (Lefaucheur et al., 2014; Noda et al., 2015; Peters et al., 2016).

There are many ways to deliver TMS, which will have consequences on current density generated into the brain and on the extent of biologic action. Some physical parameters that affect current density include the type of magnetic coil on the device, how the coil is oriented with respect to the head, and how far the coil is from brain tissue. Some parameters that affect the biologic effects of TMS include the shape of the magnetic pulse waveform, the intensity, the frequency and pattern of stimulation, and the geometric orientation of current lines with respect to the excitable neural elements (Lefaucheur et al., 2014). Moreover, TMS may be delivered as single or paired pulses or as regularly repeating pulses (rTMS). Studies of the therapeutic potential of TMS for OCD generally employ rTMS, given that repeated TMS may be needed to produce neural effects that outlast the duration of stimulation.

Since the earliest report of rTMS in OCD (Greenberg et al., 1997), much of the empirical effort has been focused on the identification of stimulation parameters and cortical targets of rTMS yielding a therapeutic effect in OCD. The latest evaluation of those efforts is contained in a meta-analysis of 18 randomized and sham-controlled trials published up to December 2016 (Rehn et al., 2018), which also provides an update on three earlier meta-analyses (Berlim et al., 2013; Trevizol et al., 2016; Zhou et al., 2017).

In the reported meta-analysis (Rehn et al., 2018), there were 484 subjects with OCD (262 were randomized to active and 222 to sham rTMS). Most patients had some degree of treatment resistance so that rTMS was used as an augmentation strategy. Active rTMS was found to be superior to sham rTMS in the treatment of OCD, producing a modest effect in reducing YBOCS scores. It was also found that rTMS over the supplementary motor area produced a greater reduction in YBOCS scores than rTMS applied over the dorsolateral prefrontal cortex or orbitofrontal cortex (OFC). Notably, subgroup analyses also indicated that the effectiveness of rTMS was greater at 12 weeks than at 4 weeks of follow-up. Nevertheless, the authors cautioned that given the still relatively small number of trials and the variety of rTMS protocols employed, "the clinical utility of rTMS in the treatment of OCD requires further investigation as to the most optimal stimulation parameters" (Rehn et al., 2018, p. 661).

What mechanisms underlie the effectiveness of rTMS in reducing OCD symptoms? Like for psychosurgery and DBS (Bear et al., 2010), it is considered that the therapeutic effect stems from a manipulation of the cortico-striato-thalamo-cortical (CSTC) circuits. However, there is another potential component to the beneficial effects: rTMS has been conceptualized as a procedure for manipulating brain plasticity (Pascual-Leone et al., 2005; Hallett, 2007). Hence, it is supposed that rTMS may create the neurobiological condition enabling new learning to stop performance of compulsions; consequently, rTMS is often applied during provocation of OCD symptoms as a variant of the clinically used "exposure-response prevention" paradigm (Carmi et al., 2018). Other possible effects of rTMS that may contribute to its therapeutic efficacy are similar to the considerations raised earlier for DBS, namely, that "rTMS can interact with spontaneous oscillatory rhythms existing in the cortical circuits activated by the stimulation" (Lefaucheur et al., 2014, p. 2154) and that the effectiveness of rTMS over the supplementary motor area may be related to findings that the beneficial effects of STN DBS may require activation of the cortical afferents to STN (McIntyre and Hahn, 2010).

b. Transcranial Direct Current Stimulation. Whereas rTMS can generate neuronal action potentials, tDCS cannot; however, the application of the direct current transcranially is of sufficient magnitude to shift the resting membrane potential of neurons toward depolarization or hyperpolarization and thereby alter cortical excitability and neuroplasticity (Lefaucheur et al., 2017). Notably, the neuroplastic action of tDCS is diminished by blockade of $N$-methyl-D-aspartate (NMDA) receptors (Lefaucheur et al., 2017). The appeal of a tDCS device includes its relative simplicity and low cost (it may be bought freely on the internet) and the possibility that it could be used by the patient at home under remote supervision (Palm et al., 2018). However, there are no established guidelines how to apply tDCS and this is of clinical importance because there are many ways to deliver tDCS with consequences on the biologic effects produced and how long they persist (Nitsche et al., 2008; Senço et al., 2015; Lefaucheur et al., 2017). Although a "definite efficacy" of tDCS has not yet been established for any indication, there is evidence of "probable efficacy" for fibromyalgia, depression, and craving (Lefaucheur et al., 2017).

tDCS has also been investigated for its therapeutic potential in OCD, based partly on the notion that it can be an effective procedure to "activate most of the [brain] areas related to OCD" (Senço et al., 2015, p. 381). A recent systematic review of the literature (Brunelin et al., 2018) identified 12 studies for qualitative evaluation, with only one being a randomized controlled study (D'Urso et al., 2016); none of the studies included a sham procedure. The review included a total of 77 patients who "received active tDCS with a large diversity of electrode montages mainly targeting the dorsolateral prefrontal cortex, the orbitofrontal cortex or the (pre-) supplementary motor area." Most of the studies reported a decrease in YBOCS score, and there were also "beneficial effects of tDCS on other symptoms that are often observed in patients with OCD, such as depression and anxiety" in some studies (Brunelin et al., 2018). The authors conclude that "tDCS appears to be a promising tool to decrease 
obsessive-compulsive symptoms," although clearly further studies are needed, including the evaluation of appropriate stimulation parameters and electrode placements (Brunelin et al., 2018).

4. Neuromodulatory Therapy Combined with Other Forms of Obsessive-Compulsive Disorder Therapy. Given the relative newness of most of the neuromodulatory interventions reviewed above, there are insufficient studies comparing neuromodulatory treatment as monotherapy against the combination with another treatment of OCD. Moreover, surgical interventions are usually reserved for treatment-refractory OCD, and these patients generally receive concurrent pharmacotherapy and/or CBT treatments in addition to the intervention being evaluated. For example, in the study evaluating the effectiveness of DBS, patients continued with their usual medications and had multiple weekly sessions of CBT together with DBS (Denys et al., 2010; Mantione et al., 2014). Similarly, in a metaanalysis of rTMS studies, the inclusion criteria included rTMS "either as monotherapy or as an augmentation strategy for OCD" (Rehn et al., 2018, p. 649). Hence, at present there are insufficient data to assess the clinical advantage of neuromodulatory therapy by itself versus in combination with other forms of OCD therapy.

\section{Psychologic Treatments for Obsessive- Compulsive Disorder}

Pharmacotherapy and neuromodulation therapy both use physical agents to directly target brain neurochemistry and functional neuroanatomy. In contrast, psychologic intervention or "psychotherapy" is a nonphysical treatment modality with demonstrated empirical evidence of therapeutic benefit in OCD, as mentioned earlier. There are two fundamental sorts of such intervention, emanating from within different fields of psychology and ostensibly targeting a different aspect of the OCD condition. One of these is behavior therapy and the other is CT. We describe both here and then summarize the empirical evidence for their efficacy in the treatment of OCD and compare them with pharmacotherapy treatments. We also highlight the empirical findings from these interventions that we consider may provide clues as to the neurobiology of OCD. Foa (2010) provides an illuminating historical perspective on psychologic interventions for OCD and describes the two dominant forms of psychotherapy for OCD-behavioral and cognitive treatments; the article serves as an important source for the following exposition.

1. Behavior Therapy. Prior to the $1970 \mathrm{~s}$, OCD was viewed not only as refractory to pharmacotherapy (see section II.B.1) but also as intractable to psychologic treatment (Foa, 2010). Indeed, the then-available psychodynamic psychotherapy techniques (grounded in psychoanalytic and psychodynamic theories of unconscious drives) were of limited clinical benefit, leading the author of a psychiatric review on OCD to conclude that such psychotherapy procedures were ineffective for OCD and to observe that "OCD is generally easy to diagnose but extremely difficult to treat successfully" (Jenike, 1983, p. 110).

This pessimism was broken not only with the appearance of pharmacological agents to treat OCD (see section II.B.1), but coincidentally by signs of success in the treatment of OCD with behavior therapy techniques (grounded in psychologic theories of learning). As noted by Jenike (1983), "Learning theorists concentrate predominantly on the symptom itself and are largely unconcerned with underlying psychodynamic explanations" (p. 182). Various behavior therapy methods were tested to eliminate obsessions and compulsive rituals (e.g., desensitization, flooding, aversive conditioning, relaxation), but what ultimately proved efficacious is "exposure and response/ ritual prevention" (Foa, 2010).

a. Historical Roots of Exposure and Response/Ritual Prevention Therapy. Levy and Meyer previously described a successful behavior modification treatment of OCD (Meyer, 1966; Levy and Meyer, 1971). Hospital inpatients, under constant supervision by the nursing staff, were prevented from performing their rituals through the following:

... a variety of ways which included engaging the patient in other activities, discussion, sometimes cajoling and very occasionally the use of mild physical restraint which was resorted to only with the patient's agreement.

When total prevention of the rituals had been achieved, the continuous supervision was maintained for a period varying between 1 and 4 weeks during which the patient was gradually exposed to situations which previously evoked rituals and was again prevented from carrying these out. Avoidance of such things as door-handles was dealt with by persuasion and occasionally by placing the patient's hand on the avoided object. Supervision was then gradually diminished until the patient was totally unsupervised but occasionally observed. Recurrences during the period of observation were taken as an indication to resume supervision. (Reprinted from Levy and Meyer, 1971, p. 30)

All patients (who were symptomatic from 6 to 36 years prior to treatment) improved remarkably after the period of supervision was terminated. Moreover, at follow-up (1-6 years after completion of therapy), six of the eight patients who received this novel therapy were asymptomatic or much improved ( $>75 \%$ reduction in compulsive behavior), and two were rated as improved (50\%-74\% reduction in symptoms).

The authors coined their method "apotrepic therapy" (from Greek for to turn away, deter, or dissuade), rather 
than "response prevention" (Levy and Meyer, 1971), because at the time they "could not pin-point the relevant aspects of the treatment" (Meyer, 1966, p. 279). Nevertheless, inspired by the success of this therapy, other clinicians conducted controlled treatment studies that included some variation on the method described by Levy and Meyer (1971) but which usually retained two components of their method: "exposure in vivo" (i.e., repeated contact with items provoking obsessive thoughts and compulsive behavior) and "ritual prevention" (i.e., repeated practice to refrain from performance of compulsive behavior). In a study with three groups of OCD washers where one group received "exposure in vivo" only, another group received "ritual prevention" treatment only, and the third group received both treatments, it was found that the group receiving the combined treatments benefited the most (Foa et al., 1984). Hence, most behavior therapy methods contain those two components, with the procedure known today as ERP.

b. Description of Exposure and Response/Ritual Prevention Therapy. In actual clinical practice, there are a number of ways in which ERP therapy may be administered. Those that adhere closely to using predominantly only the "in vivo exposure" and "response/ritual prevention" components are considered as "behavior therapy." When ERP therapy includes additional components, it is generally referred to as a variant of CBT, or just CBT. One such highly successful and empirically evaluated CBT "package" is described in Foa (2010), who indicates that the ERP treatment program (used in their practice and in clinical trials) includes the following four components: in vivo exposure, imaginal exposure, ritual prevention, and processing:

Exposure in vivo (i.e., exposure in real life), involves helping the patient confront cues that trigger obsessive thoughts. Cues include objects, words, images, or situations. For example, touching water faucets in a public restroom might trigger germ obsessions. Cues [are] presented in a hierarchical manner, beginning with the moderately distress-provoking ones and progressing to more distressing cues.

Imaginal exposure involves asking the patient to imagine in detail the distressing thoughts or situations. ... For example, imaginal exposure may involve the patient imagining contracting a sexually transmitted disease because they did not wash their hands sufficiently after using a public bathroom and consequently being shunned by friends and family....

Ritual prevention involves instructing the patient to abstain from the ritualizing that they believe prevents the feared disaster or reduces the distress produced by the obsession (e.g., washing hands after touching the floor and fearing contracting a disease)....
"Processing involves discussing the patient's experience during or after exposure and response prevention, and how this experience confirms or disconfirms the patient's expectation (e.g., you touched the floor and you did not wash your hands for about 1 hour; is your level of distress as high as in the beginning of the exposure? How strong are your urges to wash? Are they as strong as you expected? If not, what have you learned from this experience?)....

The bulk of the treatment program involves the practice of exposure and ritual prevention exercises, both in [therapy] session and as homework assignments, working through more difficult exposures as treatment progresses. During the last few sessions, emphasis is placed on relapse prevention and future maintenance of gains. These sessions can be conducted either once a week, twice a week, or daily in an intensive treatment program, depending on symptom severity and logistical considerations. (From Foa, 2010, pp. 201-202, CC BY-NC-ND 3.0)

There have been intense efforts to empirically evaluate the parameters of these components for optimal clinical benefit and we will consider the relevant empirical evidence later, after describing the other dominant psychologic intervention, namely CT.

2. Cognitive Therapy. The roots described earlier for behavior therapy stem from theories of learning that were grounded in the then-dominant tradition of psychology, behaviorism, which is "a view that virtually denied the existence of mind" (Thagard, 2014), and restricted psychology to the study of the "relation between observable stimuli and observable behavioral responses" (Thagard, 2014). The roots of CT do not lie in behaviorism denying the mind but in a field that is quite the opposite. In the 1960s, recognition of the inadequacies of behaviorism (Chomsky, 1959) led to the growth of a scientific pursuit where "researchers in several fields began to develop theories of mind based on complex representations and computational procedures" (Thagard, 2014). One of these scientific fields was cognitive psychology, studying how mental processes (cognitions) affect behavior; CT emerged from such studies and the associated cognitive theories (https://en.wikipedia.org/wiki/ Cognitive_psychology).

The theoretical framework adapted in CT is that thought processes drive OCD ("antecedent cognitions"; Tolin, 2009). Accordingly, CT is a method of "cognitive restructuring." Foa (2010) describes a CT treatment program as follows:

In the first stage of CT, patients are taught to develop an awareness of their worries as obsessions and their rituals as compulsions. The patient keeps a daily diary of obsessions, called a thought record. In the thought 
record, patients write down their obsessions and the interpretations associated with the obsessions. Important details to record may include what the patient was doing when the obsession begin, the content of the obsession, the meaning attributed to the obsession, and what the patient did in response to the obsession (usually a compulsion).

The therapist will review the thought record with the patient and how the obsession was interpreted. Using gentle reasoning and Socratic questioning, the therapist will verbally challenge an unrealistic belief. This helps the patient to identify the cognitive distortion, typically a faulty assessment of danger, an exaggerated sense of responsibility, or fears that thinking something negative will make it come true (thought-action fusion).

Once patients are able to quickly identify their obsessions and compulsions as symptoms of OCD, the therapist will initiate a few behavioral experiments to disprove errors in thinking about cause and effect. For example, if a patient believes that smoking four cigarettes will prevent her family from being harmed in an auto accident, the therapist may instruct the patient to smoke only three cigarettes and then wait to see if family members are actually harmed that day in an auto accident. The therapist may then use the results of this experiment as material for discussion about other types of magical thinking. Over time, patients learn to identify and re-evaluate beliefs about the potential consequences of engaging in or refraining from compulsive behaviors and subsequently begin to eliminate compulsions (see ref [Salkovskis, 1998]). (From Foa, 2010, p. 204. CC BY-NC-ND 3.0)

As for ERP therapy, there are variations in the protocols of CT. Those with a sizeable component of "behavioral experiments" (which resemble ERP practice) are labeled CBT.

3. Efficacy of Psychologic Intervention. A number of reviews and meta-analyses are available that evaluate and compare the efficacy of psychologic treatments for OCD (National Collaborating Centre for Mental Health, 2006; Rosa-Alcázar et al., 2008, 2015; Ougrin, 2011; Olatunji et al., 2013; Katzman et al., 2014; Öst et al., 2015; Skapinakis et al., 2016b). They establish that psychotherapy can result in marked clinical benefit, although it is less clear whether differences exist among the various forms of psychologic treatment and whether psychologic treatment is more or less efficacious than pharmacotherapy, either as monotherapy or in combination. We first highlight the evidence for the efficacy of psychotherapy and then in combination with medication.

\section{a. Exposure and Response Prevention, Cognitive Therapy,} or Cognitive-Behavioral Therapy as Monotherapy. In a review of the evidence used by four leading international organizations [the National Institute for Health and Care Excellence, Division 12 (Clinical Psychology) of the American Psychological Association, Cochrane, and the Australian Psychological Society] to recommend psychologic treatments for mental disorders, there was "excellent agreement," with all four asserting high quality of evidence for the efficacy of CBT (Moriana et al., 2017). Furthermore, the authors noted that Division 12 (Clinical Psychology) of the American Psychological Association is "the only organization that differentiates exposure and response prevention (ERP) from CBT, rating both therapies as having strong level of evidence. In contrast, ERP is included within the concept of CBT by National Collaborating Centre for Mental Health (2006), Cochrane (Gava et al., 2007), and Australian Psychological Society (APS) (2010)" (p. 35).

How large is the therapeutic effect obtained with CBT? In a most recent meta-analysis of psychologic and pharmacological interventions for OCD, treatment efficacy for CBT (assessed as the mean YBOCS score reduction from baseline compared with drug placebo) was -7.98 with a $95 \%$ credible interval of -11.02 to -4.93 (Table 2 in Skapinakis et al., 2016b). The same meta-analysis included, as separate categories, psychotherapeutic studies evaluating the efficacy of "behavioural therapy [BT], including exposure and response prevention but not explicit cognitive techniques (such as cognitive restructuring); cognitive therapy $[\mathrm{CT}]$, including cognitive restructuring but not explicit behavioural techniques; and cognitive behavioural therapy (CBT)" (Skapinakis et al., 2016b, p. 731). The magnitudes of the therapeutic effects for these two categories were comparable and not different from the effects of CBT, with mean (95\% credible intervals) of $-10.41(-14.04$ to -6.77$)$ for the BT intervention and $-9.45(-13.76$ to -5.19$)$ for the CT intervention. $^{1}$

Other meta-analyses also did not identify differences between these forms of psychotherapy. For instance, in a systematic review and meta-analysis

\footnotetext{
${ }^{1}$ The cited values for treatment efficacy exclude "waiting list" controlled trials. Table 2 in the meta-analysis by Skapinakis et al. (2016b) for treatment efficacy also provides values based on an analysis of the "full network," which includes "waiting list" trials. Others have shown that the estimates of effect size (ES) for psychotherapy trials are larger ("inflated") when the study utilizes a "wait list" control condition rather than some other active control condition, attributed to a "performance bias" (e.g., Skarphedinsson et al., 2015). For the "full network" analysis, their Table 2 shows mean values of greater efficacy for BT $-14.48(-18.61$ to -10.23$)$ and for CT $-13.36(-18.40$ to -8.21$)$, but not for CBT $-5.37(-9.10$ to -1.63$)$.
} 
of studies published between 1993 and 2014 on the efficacy of cognitive-behavioral treatments for OCD (Öst et al., 2015), similarly large ESs compared with placebo were found for CBT, ERP, and CT. To confirm this apparent lack of difference between the psychotherapies, the researchers identified in their meta-analysis six studies (van Balkom et al., 1998; Cottraux et al., 2001; McLean et al., 2001; O'Connor et al., 2005; Whittal et al., 2005; Belloch et al., 2008) that permitted a direct comparison of ERP with CT. Analysis of these studies found no difference in the efficacy of ERP and $\mathrm{CT}$, as measured by the change in YBOCS scores from pre-treatment to post-treatment and follow-up. The authors concluded that "modern forms of CT and ERP do not differ significantly in clinical outcome" (Öst et al., 2015, p. 164).

As may be garnered from the descriptions of ERP and CT protocols (see section II.D.1.b) and section II.D.2), they both have components in common, despite seemingly different theoretical frameworks on OCD. Rosa-Alcázar et al. (2008) conducted a meta-analysis of 19 studies published between 1980 and 2006 and concluded that ERP and CT are "equally effective in the treatment of OCD and that the combination of the two techniques did not seem to produce an improvement in effectiveness over and above that showed by the two techniques separately" (p. 1320). The authors speculated the following:

The reason that ERP and [CT] are so similar could be due to the fact that both techniques incorporate similar treatment strategies. Thus, for example, the application of $[\mathrm{CT}]$ often involves behavioral experiments that include exposure to anxiety-evoking situations to negate their irrational thoughts, so [CT] also incorporates behavioral as well as cognitive components. On the other hand, the application of ERP involves arguing with the patient about his/her beliefs on fear and his/her irrational thoughts on decision-making under uncertainty, so ERP contains its own elements of cognitive therapy. (From Rosa-Alcázar et al., 2008, p. 1321, with permission from Elsevier.)

In another analysis of which components in ERP and CT may be truly efficacious for OCD, Tolin (2009) argues that what is most likely responsible for improvement is "interventions that encourage direct behavioral change (described as a characteristic feature of ERP and ... an incidental feature of CT) ..., whereas evidence for the importance of altering antecedent cognitions (a characteristic feature of CT and an incidental feature of ERP) is less clear" (Tolin, 2009, p. 40). However, to identify which aspects of the therapy protocols are truly the necessary or sufficient components for best clinical benefit will require controlled research studies, which are difficult to perform.
One reason why controlled research studies in psychotherapy are difficult to design and implement relates to specifying the appropriate control condition (Kirsch, 2005). For instance, testing which aspects of the psychotherapy session constitute the theoretically specified mechanism of the treatment effect presupposes that the investigator can specify the "incidental features" of the therapy for assignment to the control condition. Generally, what is "important" and what is "incidental" in the psychologic intervention is usually opaque to the therapist, as it is to the patient.

b. Timing of Psychotherapy Sessions. Nevertheless, one important question (whether a spaced or a massed schedule of therapy sessions is more efficacious) seems relatively simple to answer empirically. The question is important because knowing the relationship between therapeutic efficacy and timing of sessions provides guidance for optimizing the allocation of clinical resources. Moreover, the information is also revealing for OCD theory.

There are two types of CBT treatment schedules in clinical practice: "intensive" and "regular" ("standard"). The intensive schedule typically is a "massed" protocol, with a daily regimen of sessions during the first 3 to 4 weeks of treatment, such as in the study by Foa et al. (2005) described in section II.B.3. The "regular" or "standard" schedule typically is a "spaced" protocol, where sessions with the therapist are at weekly or twice-weekly intervals. There are several studies and one meta-analysis evaluating the efficacy of these treatment schedules, all agreeing that both schedules are efficacious but of mixed evidence whether massed trials are superior to spaced sessions.

The one meta-analysis (Jonsson et al., 2015) was undertaken in light of the already established efficacy for regular CBT but lacked corresponding evidence for intensive CBT. Jonsson et al. (2015) identified 17 trials (11 adult and six youth; total of 646 participants) with an "intensive CBT format defined as (a) no more than 4 weeks total duration, (b) a minimum of 10 therapist hours in total, and (3) at least 5 therapist hours per week on average." Based on ES as the index of efficacy comparing pre- and post-treatment measures of OCD (generally YBOCS scores but not in every study), there was a large overall pre-post ES of 2.44 (95\% confidence interval, 2.03-2.85), and the authors noted that "the overall pre-post ESs of this meta-analysis are larger than previously reported estimates from meta-analyses of standard individual or group CBT and suggest some superiority of the intensive format" (Jonsson et al., 2015, p. 93).

The claim for a "superiority" of the intensive CBT format over the regular weekly schedule was supported by between-group comparisons of ES statistics from three studies with both intensive and standard CBT (Abramowitz et al., 2003; Storch et al., 2007, 2008). There was a significant between-group ES of 
0.37 (95\% confidence interval, 0.02-0.71) in favor of the intensive treatment condition compared with standard CBT (Jonsson et al., 2015). However, this evidence favoring intensive CBT turned toward being "mixed" when the comparison at 3-month follow-up was included; ES statistics for intensive and standard CBT were statistically not different at follow-up (Jonsson et al., 2015). Interestingly, the researchers noted that this loss of difference at follow-up appeared related to a "slight deterioration in the intensive CBT format while patients from weekly conditions changed little over the follow-up period" (Jonsson et al., 2015, p. 94), which was most evident in the study by Abramowitz et al. (2003).

It is not known why there was deterioration at 3month follow-up after intensive CBT in the study by Abramowitz et al. (2003). Nonetheless, the evidence seems strong that massed or intensive CBT can reach maximum symptom relief in a much shorter period of time than standard CBT. In fact, there are reports of CBT treatment concentrated into a 4-day period-the Bergen 4-day concentrated exposure treatment for OCD - that is highly effective. A recent publication (Hansen et al., 2018) reported YBOCS scores from 65 patients with OCD who underwent this treatment with follow-up at 3 and 12 months. Their mean \pm S.D. YBOCS score was $25.83 \pm 4.65$ at the start of treatment and declined to $10.24 \pm 5.13$ at the end of treatment; it was $10.45 \pm$ 5.863 months later and was similarly low at 12 months $(10.64 \pm 7.00)$. These YBOCS scores in reduction of symptoms were significantly lower than observed with standard ERP, using the data from randomized trials reported in the meta-analysis of Öst et al. (2015). Moreover, using a YBOCS score of $\leq 11$ as the criterion for remission/recovery, $64.6 \%$ of the 65 patients showed remission/recovery at 1 -year follow-up compared with $44.2 \%$ of 104 patients who received standard ERP in the meta-analysis of Öst et al. (2015), a difference that was statistically significant $(P=0.01)$. The authors report that there was $0 \%$ dropout from this treatment and conclude that the "Bergen 4-day treatment is a feasible way to deliver treatment for OCD, and the effects are stable at 12-month follow-up" (Hansen et al., 2018).

Thus, given successful replication of the Bergen 4-day treatment efficacy, this would constitute very strong evidence that massed or intensive CBT treatment results not only in quicker relief of OCD symptoms than standard ERP but also that those clinical gains are sustained for at least 1 year.

4. Efficacy of Cognitive-Behavioral Therapy with or without Pharmacotherapy. The evidence reviewed earlier strongly supports the efficacy of CBT in the treatment of OCD. Hence, clinical guidelines include CBT as one of the recommended first-line treatment options. Moreover, said efficacy is even used to formulate public health policy for delivery of mental health services (Clark, 2018). However, it is not established empirically whether psychologic or pharmacological intervention is superior or even if psychotherapy combined with pharmacotherapy provides added benefit in the treatment of OCD (section II.B.3). It could be argued that such studies to identify evidence-based best treatments are not even feasible in clinical practice because with an empirically driven trial-and-error approach, the parameter space to investigate is just too large. This problem is compounded by the reality of clinical practice where at least half of the participants entering a randomized clinical trial generally have received or are currently taking medication (Skapinakis et al., 2016b), precluding the evaluation of "pure" psychotherapy and pharmacotherapy effects.

For instance, a recent meta-analysis (referred to in sections II.B.3 and II.D.3.a) of the relative efficacy of various psychologic and pharmacological OCD therapies found that ERP was more effective than SRI pharmacotherapy (Skapinakis et al., 2016b). In that study, which involved 53 published articles spanning the years 1980-2012, ERP resulted in a mean YBOCS score reduction of $-10.41(-14.04$ to -6.77$)$ compared with drug placebo. By contrast, clomipramine and SSRI treatments were associated with reductions of $-4.66(-6.26$ to -3.05$)$ and $-3.62(-4.89$ to -2.34$)$, respectively (Skapinakis et al., 2016b). However, the authors discounted these differences by noting that most of the studies that employed psychotherapy involved symptomatically stable patients who were also receiving pharmacotherapy; furthermore, studies that stated explicitly that psychotherapy was administered in the absence of pharmacotherapy were relatively uncommon (Skapinakis et al., 2016b). Conversely, others have suggested that pharmacotherapy trials may covertly include participants receiving concurrent formal or informal forms of psychologic intervention (Johnson et al., 2016).

Similarly, even though in their meta-analysis Skapinakis et al. (2016b) concluded that "the combination of psychotherapeutic and psychopharmacological interventions is likely to be more effective than are psychotherapeutic interventions alone," others believe this conclusion goes beyond the data and as such is potentially harmful in the guise of an evidence-based treatment recommendation (Wheaton et al., 2016a). In their response, the authors agree and point out the multitude of issues in making inferences from clinical trials (Skapinakis et al., 2016c).

Wheaton et al. (2016a) summarize the following conclusions from the available studies on efficacy of various treatments: 1) medications and CBTs are both reasonably effective treatments for OCD; 2) although some patients may benefit from the combination of medication and CBT, the available empirical evidence has not established its superiority; and 3) "starting with psychotherapy is a rational first 
step for some patients to minimise unnecessary costs and risks of medication" (p. 920). Unfortunately, there is currently no theoretically grounded rationale for determining which type of treatment is indicated for particular subgroups of individuals with a diagnosis of OCD.

5. Mechanisms of Cognitive-Behavioral Therapy Therapeutic Effect on the Obsessive-Compulsive Disorder Condition. What are the mechanisms for the therapeutic effect of CBT in OCD? The very fact that CBT is efficacious indicates unequivocally that this disorder is sensitive and responsive to psychologic intervention (i.e., to perturbation of the nervous system with agents such as "words" and "actions," which physically and directly have no active medicinal ingredients). But it is not words and actions as such that constitute the healing elements of CBT. Rather, as noted by Kirsch (2005) for psychotherapy in general, "it is their meaning... a patient's response to the meaning of a treatment... to its psychological properties rather than its physical properties" (p. 797).

What are those psychologic properties of CBT which account for its efficacy in OCD? Note that this is not a question of whether CBT is efficacious for OCD; research has established that this treatment is effective. Yet research on efficacy did not investigate mechanisms. A different type of research is needed to investigate what psychologic mechanisms underlie the effectiveness of CBT, and yet such studies are scarce for at least two reasons.

First, for a long time, the efforts of the clinical field were focused on evaluating the efficacy of psychologic interventions and identifying the ingredients for a clinically effective psychologic treatment regimen. Studies using randomized clinical trials to establish ERP and CT as evidence-based treatments for OCD were simply not designed to investigate the mechanisms underlying their effectiveness. Similarly, empirical studies comparing intensive versus standard CBT indicated that a massed program may be more efficacious but did not address why. Likewise, the "dismantling studies" reviewed by Foa (2010) were designed to identify which specific components of the ERP package provide clinical benefit for treating OCD, but they did not investigate the underlying psychologic properties that would explain this effectiveness. In short, for many decades, the priority of research effort was understandably on evidence for treatment efficacy, not underlying mechanisms.

Second, there was insufficient appreciation that studies of a different sort are required to identify mechanisms. Historically, psychotherapy outcome research seems to have adapted the same problematic approach as pharmacotherapy research. As described in section II.B.1, researchers used the mode of action of clinically effective drugs to infer the neurobiology of OCD as well as its etiology and pathophysiology. However, as already noted in the context of that pharmacotherapy research, this line of reasoning was "fraught with problems that have plagued the field of psychiatry for decades ... [and] has yielded few new insights or novel treatments" (Goodman et al., 2014, p. 261), not least because deductions of etiology from findings of treatment effects are a logical fallacy, the "fallacy of confirming the consequence" (Montgomery, 2012). Nonetheless, psychotherapy research had followed the same trajectory, using the success of psychologic interventions such as ERP and CT as evidence for theories of OCD.

For example, ERP therapy arose as a behavior modification procedure within conditioning theory (section II.D.1). Hence, in ERP, principles from learning theory are applied to teach the patient, using various methods, not to respond to compulsion-evoking stimuli with the usual compulsive behavior. One such important teaching method is the practice of withholding compulsive rituals ("prevention") to presentations of compulsionevoking stimuli ("exposure"). Accordingly, the psychologic mechanisms for ERP effectiveness in OCD are learning: learning to break the (putative) association between compulsion-evoking stimuli ("obsessions") and compulsive rituals ("compulsions"), and learning that this break has no negative consequences. By the same token, the inference from the success of ERP was a conditioning account of OCD symptoms, where OCD was "acquired through classically conditioned fear responses, and maintained through negatively reinforced avoidance responses" (McKay et al., 2015, p. 106) and where those learned OCD associations could be reconditioned to produce symptom-free responses.

Likewise, CT emerged from within cognitive psychology (see section II.D.2) where one viewpoint held that "cognition precedes behavior" (McKay et al., 2015, p. 107), with higher-order cognitive processes, such as thinking, beliefs, and appraisals, driving behavior directly. Hence, in CT, various techniques of "cognitive restructuring" are used to modify the cognitions that drive obsessions and compulsions to yield symptom-free behavior. Among those techniques are gentle reasoning and Socratic questioning, contesting unrealistic beliefs, and "behavioral experiments to disprove errors in thinking about cause and effect" (Foa, 2010, p. 204). Accordingly, the psychologic mechanisms for the effectiveness of CT in OCD are persuasion-the remodeling of thinking, beliefs, and appraisals, to drive behavior without producing obsessions and compulsions. By the same token, the inference from the success of CT is a purely cognitive account of OCD, where obsessions and compulsions are produced by faulty reasoning and where elimination of these symptoms is produced through presentation of evidence and sheer force of argument.

These presumed theories of OCD are not tested but taken as a given in CBT. Indeed, all forms of CBT begin with "psychoeducation," where the psychotherapyrelevant theory of OCD is described and used to explain 
the ensuing treatment. To the patient, psychoeducation material likely appears as established knowledge about the disorder. As such, it provides a framework for the patient to recognize the psychologic meaning of the various procedures and favors adherence rather than questioning the value of the administered intervention. Psychoeducation probably constitutes a crucial psychologic ingredient for CBT effectiveness.

Yet the success of CBT is not a test of the theory about the disorder. As an example, this can be seen in the clinical literature from the type of interpretation considered when CBT is not effective. If CBT were also a test of the theory, then treatment nonsuccess should raise as one possibility a consideration of problems with the theory, but the usual considerations are of failures to protocol adherence. Thus, it had been found that adherence to therapy protocol is a good predictor of therapeutic outcome (Wheaton et al., 2016b) and conversely that not following the prescribed components "hinders outcome" (McKay et al., 2015). In the clinical literature, the most common explanation for this relationship is low ability to confront triggers of OCD symptoms and/or low ability to refrain from performance of compulsions. Such patients are considered to subterfuge exposure through various tricks such as "covert" rituals, denying the therapeutic benefits of ERP; moreover, it is considered that such patients are more likely to refuse treatment (McKay et al., 2015). Similarly, another common explanation for hindrance of outcome is the possession of "overvalued ideas" (Markarian et al., 2010). Some patients are considered to have "a pathologically extreme belief in the realistic aspect of thoughts" and view obsessions as "reasonable." This is considered to create a barrier to interventions that conceptualize "the disorder as based on primary cognitive errors that can be challenged" and frank acceptance by the patient that "the link between obsessions and compulsions is illusory" (Markarian et al., 2010, p. 82). However, this same literature rarely considers an alternative possibility for refusal of CBT treatment or nonadherence: the psychoeducation material describing the reasons for OCD does not resonate with self-knowledge for this behavior, putting into question the psychologic meaning of the interventions and thereby exposing key procedures as truly not compelling or persuasive. Stated in another way, if success of CBT were also a test of the theory, then it should be considered that at least some aspects of the theory on which the rationale for the therapy is said to depend-for example, "recognition that the link between obsessions and compulsions is illusory" (Markarian et al., 2010)—may be the wrong formulation of the OCD condition.

For CBT, there is a lack of empirical evidence regarding the underlying mechanisms by which it is efficacious for OCD, a state of affairs that, as we have seen, also characterizes pharmacological and neuromodulator therapies for OCD (sections II.B and II.C). However, a hypothesis that considers and encompasses all of the effective therapies can be suggested: By different means, effective interventions (pharmacotherapy, neuromodulation treatments, and CBT) alter the psychologic meaning of the offending situation, thereby favoring other than OCD behavior. In particular, pharmacological intervention acts directly on signal transduction processes to engage synaptic plasticity and thereby enable the shift in processing of the offending inputs to yield symptom-free responses. Similarly, neuromodulation treatments engage neural plasticity through direct electrical stimulation of key neuroanatomical sites. Finally, CBT uses psychologic means to set up the conditions for neural plasticity and engage learning that alters the psychologic meaning of the offending situation and thereby favors non-OCD behavior.

\section{E. Summary}

The diagnosis and description of OCD is based on descriptive phenomenology, as represented by the DSM criteria. Although it is recognized that this description is not based on knowledge of etiology or pathophysiology, potentially problematic theoretical preconceptions have tended to slip into the definition of OCD (e.g., the assumption that obsessions are the cause of compulsions). Because categorical diagnosis of OCD is too coarse as a basis for research on treatment outcome, multi-item rating instruments have been developed based on the OCD criteria, the most widely used of which is the YBOCS, which yields a useful quantitative index of the severity of OCD symptoms. Unfortunately, there has recently been a growing chorus of criticism of the DSM as the longstanding foundation for research in psychopathology, because this approach has generally failed to lead to solid advances in etiology and theory. We take up the prospect of alternative approaches to research on OCD later in section IV.

Research shows that a variety of treatments (pharmacological, neuromodulatory, and psychotherapeutic) are effective for OCD; however, this research does not illuminate why these treatments work. Although it has sometimes been tempting to use findings of treatment effectiveness to make inferences about the etiology of OCD, such inferences are tentative and problematic (just as it would be faulty to deduce that headache is caused by lack of aspirin). However, it is an important long-term goal for OCD theory to explain why various treatments are effective, including the underlying mechanisms. More generally, investigating theories of OCD, including etiology and pathophysiology, requires a basic science approach in which putative causes can be manipulated experimentally. This is a major theme to which we turn later in section $\mathrm{V}$.

In addition to the results of research on treatment of OCD, there is a very wide range of other research 
findings pertaining to OCD in humans. Because the ways in which OCD is conceptualized and measured are more varied in this research, it is important to attend carefully to this aspect of the studies. In addition, these research findings are diverse and sometimes confusing; nonetheless, they may provide some potentially important clues about the underlying nature and pathophysiology of OCD. We review this large body of work in section III.

\section{Current Research on the Neurobiology of Obsessive-Compulsive Disorder}

Currently, ongoing research on the neurobiology of OCD can be conceptualized as focused on two interrelated major objectives. One of these major objectives is to identify the neurobiological fault that underlies OCD so that appropriate neuroscience methods can be developed to repair or ameliorate this fault. Another major objective is to identify the etiology of OCD-the factors that lead to the underlying neurobiological fault in the first place.

Much of this research takes as its starting point the clinical findings described in section II and investigates at the molecular, cellular, or systems levels whether those efficacious treatments identify a neurobiological fault yielding OCD. This research also probes for faulty neurobiology with other methods. For example, one method is to administer a very specific neurobiological challenge and look for an effect on the OCD condition; evidence of such an effect is then taken as indication of a plausible link between the neurobiological target of the challenge and OCD. Another method involves the measurement of various neurobiological factors at the molecular, cellular, or systems levels and looking for the presence of an association with the OCD condition; such a correlation suggests a potential lead that this molecular, cellular, or systems factor is a constituent of the faulty neurobiology, to be investigated further. All of these types of research involve both human and animal studies, with animal models of OCD playing a crucial role in examining hypothesized mechanisms. Here we review work probing a wide range of factors as possible leads in OCD research: neurotransmitters, including serotonin, dopamine, glutamate, and GABA, and other endogenous variables, including oxidative stress, second-messenger precursors, the microbiome, and neurosteroids and neuropeptides. We also review brain imaging studies that measure correlations between regional brain activity and OCD symptoms as potential leads for revealing the faulty neuroanatomy underlying OCD.

Research on the etiology of OCD (covered in section III.D) searches for naturally occurring factors associated with the onset or worsening of OCD. This work spans multiple levels, such as genetics, the hormonal system, and the immune system. Here, too, the rationale is to discover clues about any neurobiological fault that may underlie OCD.

\section{A. Probing the Role of Neurotransmitters in Obsessive- Compulsive Disorder}

One important avenue for research has focused on the major neurotransmitter systems in the brain in hopes of identifying a neurobiological fault yielding OCD.

1. Serotonin. As noted in section II.B.1, one of the seminal discoveries in psychiatry was the beneficial response in patients with OCD to the serotonergic TCA, clomipramine (Fernández Córdoba and LópezIbor Aliño, 1967; Ananth, 1986), which instigated the hypothesis that OCD represents a state of serotonin deficit (Bandelow et al., 2017; Sinopoli et al., 2017). Consistent with such a hypothesis, the primary target of SSRIs, the 5-HT transporter, is decreased in OCD (Matsumoto et al., 2010; Atmaca et al., 2011) and low levels are associated with increased symptom severity (Hesse et al., 2005; Reimold et al., 2007; Zitterl et al., 2008). In animal models, high stereotypic deer mice also demonstrate decreased striatal but not frontal-cortical 5-HT transporter density (Wolmarans et al., 2013). Drug challenge studies using 5-HT modulatory agents also seem to support this serotonin deficit hypothesis, although with some contradictions. On the one hand, OCD symptoms may be exacerbated by the acute administration of the nonselective $5-\mathrm{HT} 1 \mathrm{~A} / 2 \mathrm{~A} / 2 \mathrm{~B} / 2 \mathrm{C}$ agonist $m$-chlorophenylpiperazine (mCPP; Gross-Isseroff et al., 2004), an effect that is reversed by metergoline, a 5-HT antagonist (Pigott et al., 1991). Preclinical findings with the 5-HT1A agonist, 8OHDPAT (8-hydroxy-2-(din-propylamino)tetralin), support the notion that 5-HT1A agonism exacerbates OCD (Alkhatib et al., 2013). Moreover, the proposal that SSRI-induced desensitization of 5-HT1A receptors represents a basis for the anti-OCD actions of SSRIs (Piñeyro and Blier, 1999; El Mansari and Blier, 2006) is also consistent with the notion that stimulation of 5-HT1A receptors is pro-OCD. On the other hand, there is a report of a moderate effect of chronic treatment with the 5-HT1D agonist sumatriptan on obsessive-compulsive (OC) symptoms in three patients who failed to respond to prior pharmacotherapy (Stern et al., 1998). At first glance, some of these findings appear contradictory to the beneficial clinical effects of SSRIs, which in effect are indirectly acting serotonergic agents. Indeed, similar to $\mathrm{mCPP}$, acute administration of SSRIs is initially anxiogenic (Bagdy et al., 2001), often necessitating a slow upward titration of the dose in patients with OCD (Harvey, 1997). Nevertheless, 4 months of prior clomipramine treatment abrogates at least some of the behavioral effects of mCPP such as hyperthermia (Wozniak et al., 1989), suggesting that the therapeutic benefits of SSRIs involve downregulation of specific 5-HT receptors in key brain regions. An important question arising from 
this suggestion is "Which specific 5-HT receptors are implicated in OCD?"

Perhaps another clue as to which 5-HT receptors are implicated in OCD is to consider clinical observations with the multireceptor antipsychotics. These agents, such as clozapine, present with a greater antiserotonergic than antidopaminergic potency profile and have been shown to exacerbate OCD symptoms in patients with schizophrenia (Grillault Laroche and Gaillard, 2016; Leung and Palmer, 2016). Although clozapine has clinically relevant inhibitory activity at the 5-HT2A, 5-HT2C, 5-HT3, 5-HT6, and 5-HT7 receptors, it presents with agonist activity at the 5-HT1A receptors (Harvey et al., 1999), raising the possibility that modulation of 5-HT1A receptors may underlie clozapine's propensity to promote the OCD symptoms. However, the atypical antipsychotic drug and 5-HT1A receptor agonist perospirone has been found to inhibit marble-burying behavior in mice (a surrogate for compulsive behavior in an animal model) via a 5-HT1A receptor-dependent mechanism (Matsushita et al., 2005), thus contradicting this assumption. Interestingly, the 5-HT1A receptor partial agonist, aripiprazole, which clinical studies have shown to be an effective augmentation agent (Dold et al., 2015), is similarly anticompulsive but in a 5-HT1A-independent manner (Egashira et al., 2008). Although the latter finding reiterates the role of the 5-HT1A receptor in OC symptoms, it indicates that receptor state, including receptor density and sensitivity that are directly influenced by disease state, will predetermine how a 5-HT1A modulatory agent will perform. Thus, with 5-HT1A receptor density playing a crucial role in the anti-OCD actions of SSRIs (Piñeyro and Blier, 1999; El Mansari and Blier, 2006), assessment of 5-HT1A receptor response will differ if tested in a pathologic animal model versus a healthy animal model (Szechtman et al., 2017), the latter being what the two foregoing animal studies have used. In fact, in some rodent models of OCD, mCPP attenuates stereotypy (Korff et al., 2008) and checking behavior (Tucci et al., 2013, 2015). It also cannot be excluded that partial antagonistic effects at 5-HT2A and dopamine D2 receptors also underlie inhibition of marble-burying behavior by these agents. Nevertheless, modulation of 5 -HT1A receptors may represent a common action whereby the multitarget 5 -HT receptor agonist, mCPP, and clozapine exacerbate/precipitate OCD symptoms. Although animal and human data are not precisely congruent, they are unanimous in identifying the contributory role of the 5-HT1A receptor in how 5-HT is likely involved in the induction of perseverative behavior and in the pharmacologic treatment thereof. Thus, it would be reasonable to conclude that agonism of 5-HT1A receptors can either exaggerate/provoke OCD symptoms or attenuate them. Interestingly, in section II.C, we noted that lesioning a brain region can both provoke and "cure" OCD, and that both lesioning and electrical stimulation of this region can have the same effect in OCD. In a similar vein, investigators noted that the serotonin neurotransmitter system is implicated in "both the aetiology and pharmacological treatment of OCD" (Zohar and Insel, 1987, p. 325; Stern et al., 1998). This commonality across systems may provide a compelling phenomenon that is revealing of an important mechanism that warrants deeper study.

A recent study (Rodriguez et al., 2017) considered the effects of a novel 5-HT2C receptor agonist, (3S)-3methyl-1-[4-(trifluoromethyl)-7-benzofuranyl]-piperazine (CPD 1), with high potency and full efficacy at 5-HT2C receptors in three animal model preparation of OCD. One of those models (schedule-induced polydipsia in rats) relies on performance of consummatory behaviors, whereas the other two (marble burying and nestlet shredding in mice) depend on performance of nonconsummatory behaviors that are repetitive and nonhabituating. Both CPD 1 and fluoxetine suppressed marble burying and nestlet shredding, with CPD 1 dose-dependently decreasing excessive drinking engendered by a schedule of intermittent food delivery (schedule-induced polydipsia) (Rodriguez et al., 2017). The researchers concluded that the behavioral specificity of effects of CPD 1 against repetitive and excessive behaviors suggests a potential therapeutic application in OCD, thus supportive of the therapeutic utility of stimulating 5-HT2C receptors in OCD. Of interest, 5-HT2C antagonists like clozapine, but also olanzapine, paliperidone, or quetiapine, seem to be less effective augmentation strategies in OCD (Dold et al., 2015).

In summary, although it is clear that the serotonin system may have a role in both the treatment and the etiology of OCD, the specific neurobiological fault involved remains unclear and may be quite complex. Although work has pointed to the 5-HT1A and 5-HT2C receptors, the actual receptor subtypes related to the therapeutic action of SSRIs on OCD have not been clearly identified. In addition, it should be considered that manipulation of the serotonin system may affect OCD even if the principal neurobiological fault does not lie within this system.

2. Dopamine. Single photon emission computerized tomography studies have reported decreased striatal dopamine D2 receptor availability in OCD (Denys et al., 2004b), and either increased (Kim et al., 2003; van der Wee et al., 2004) or decreased (Hesse et al., 2005) striatal dopamine transporter binding. Schneier et al. (2008) showed that comorbid generalized social anxiety disorder and OCD may be associated with decreased availability of dopamine D2 receptors in the striatum. Other investigators provided suggestive evidence for an association of the dopamine D2 receptor TaqI A2 allele and the low-activity catechol- $O$-methyltransferase allele with OCD in male patients (Denys et al., 2006). In addition, increasing dopamine transmission in animals by administration of dopamine agonists or by knockdown mutation of the dopamine transporter gene provokes stereotypical behaviors that have been proposed to model 
OCD behaviors (Szechtman et al., 1998; Berridge et al., 2005).

There is also some evidence implicating D1 receptors in OCD. Positron emission tomography studies in drugfree patients with OCD have shown reduced D1 receptor binding (indicative of increased dopamine release) in the caudate, putamen, and anterior cingulate cortex (ACC) (Olver et al., 2009, 2010; Hazari et al., 2019). Functional neuroimaging studies in patients with OCD have found hyperactivation of the ACC, caudate, and putamen during rest, provocation of symptoms, or conflictinhibition tasks (Ursu et al., 2003; Mataix-Cols et al., 2004, 2005; Menzies et al., 2008; Olver et al., 2010), suggesting that dopamine, acting through D1 receptors, may modulate activity in these regions implicated in the etiology of OCD.

A number of studies in animal models have also pointed to the participation of the D1 receptor in the display of compulsive-like behavior. The most direct evidence comes from D1CT transgenic mouse lines, in which the expression of the neuropotentiating cholera toxin gene was put under the control of the D1 receptor promotor. In one of these lines in which transgene expression was restricted to the piriform cortex, somatosensory cortex, and intercalated nuclei of the amygdala, the mice displayed a number of compulsivelike characteristics, including perseveration and repetition of normal behavior patterns and repetitive leaping (Campbell et al., 1999). Further work with this model showed that chronic cholera toxin activation of these D1 receptor-expressing neurons provoked glutamatergic overexcitation of striatal output neurons of the "direct" corticostriatal-thalamocortical pathway (McGrath et al., 2000; Nordstrom et al., 2015; O'Brien et al., 2018). In other animal models, compulsive lever-pressing in the SA model was reduced by a D1 antagonist (Joel and Doljansky, 2003), whereas repetitive species-typical behavioral patterns such as grooming in the rat (Taylor et al., 2010) and nest building in the rabbit (Hoffman and Rueda Morales, 2012) were respectively strengthened by a D1 agonist and reduced by a D1 antagonist.

That SSRI-resistant OCD often responds to augmentation with a dopamine D2 receptor antagonist (see section II.B.2) suggests cooperation between serotonin and dopamine in the expression of compulsive behaviors. However, the role of dopamine is by no means clear, with OC behavior being reversed by a dopamine D2 receptor agonist and antagonist, as demonstrated in animal models (Korff et al., 2008) and humans with OCD (Fineberg et al., 2006), respectively. Moreover, although dopamine D2 receptor antagonists may benefit treatment, several studies have failed to show a hyperdopaminergic state in OCD (Pitchot et al., 1996; Brambilla et al., 2000), a result also observed in rodent models (Powell et al., 1999; Güldenpfennig et al., 2011). Furthermore, although dopamine agonists may exacerbate OC symptoms, they may also ameliorate these symptoms (e.g., bromocriptine) (Denys et al., 2004c). Similarly, opposite effects of dopamine agonists are observed in animal models of OCD. For instance, OC symptoms are provoked by the dopamine D2/D3 agonist quinpirole in the QS animal model of OCD (Szechtman et al., 1998) but are suppressed by quinpirole in naturalistic models of OCD, such as deer mouse and bank vole models (Vandebroek and Odberg, 1997; Korff et al., 2008). Such clinical and preclinical findings have led to the suggestion that an unknown form of interaction between serotonin and dopamine systems plays a role in the expression of OCD and that the effects of challenge with dopaminergic agents depends on the site of action, inherent receptor sensitivity, and the state of balance in serotonin-dopamine activity (Alkhatib et al., 2013; Johnson and Szechtman, 2016; Szechtman et al., 2017).

In an open-label trial in youths with tic disorder, the partial dopamine D2 and 5-HT1A agonist and 5-HT2A receptor antagonist, aripiprazole, yielded significant improvements in comorbid OCD, attention-deficit/hyperactivity disorder (ADHD), or depressive disorder (Murphy et al., 2009). The authors noted that these improvements in the comorbid condition may be secondary to tic reduction and/or to a specific action of aripiprazole therapy on the psychiatric disorder. Because of its dual mode of action, aripiprazole may be exerting its beneficial effect either as a full antagonist or agonist. It will be important to determine which mode of action is more dominant; such knowledge may assist in correctly interpreting dopamine-serotonin interaction in the OCD condition.

In summary, work suggests that dopamine and serotonin systems may coact in the expression of OCD. However, the specific nature of this interaction remains to be elucidated, and it is not clear that either of these systems is the principal neurobiological fault underlying OCD.

3. Glutamate and $\gamma$-Aminobutyric Acid. Magnetic resonance spectroscopy has demonstrated altered glutamate concentrations in the caudate and ACC of patients with OCD, as well as in DLGAP3 [Discs, Large (Drosophila) Homolog-Associated Protein 3] and Slitrk5 knockout mouse models (for a review, see Wu et al., 2012). Such findings have raised the suggestion that OCD may reflect disrupted glutamatergic neurotransmission within critical brain regions (Pittenger et al., 2011). Synaptic neurotransmission is controlled by synaptic glutamate levels that in turn are tightly regulated by sodium $\left(\mathrm{Na}^{+}\right)$dependent high-affinity glutamate transporters that act to remove glutamate from synapses. The glutamate transporters belong to the solute carrier 1 (SLC1) family of glutamate and neutral amino acid transporters gene group, which has five members (SLC1A3, SLC1A2, SLC1A1, SLC1A6, SLCA1A7) that encode excitatory amino acid transporters (EAATs), EAAT1- EAAT5, respectively (Hediger et al., 2013; Kanai et al., 2013; Zhou and Danbolt, 2013). EAAT3 is present in the presynaptic pool, with EAAT2 expressed at the excitatory amino acid 
synapse by surrounding astrocytes (Mathews and Diamond, 2003). Genetic linkage and association studies in OCD point to SLC1A1 (Solute Carrier Family 1 Member 1/SodiumDependent Glutamate/Aspartate Transporter 3), which encodes EAAT3 (Zike et al., 2017b). EAAT3 is found throughout the brain on the somas and dendrites of small and large pyramidal neurons (Rothstein et al., 1996; He et al., 2000; Holmseth et al., 2012; Yu et al., 2015b). Since EAAT3 is also localized to presynaptic GABA-containing terminals and may have a metabolic role in providing glutamate for GABA metabolism (Peghini et al., 1997; He et al., 2000; Yu et al., 2015b), it is important for GABAergic metabolism and hence regulation of GABA-glutamate (inhibitory-excitatory) neurotransmission in the brain. This may relate to EAAT3's pathologic role in hypoxia/ ischemia, multiple sclerosis, schizophrenia, and epilepsy (Bianchi et al., 2014), although polymorphisms of the EAAT3 gene are also implicated in OCD (Aoyama et al., 2006; Monteiro and Feng, 2016).

These findings describing the role of glutamate and its transporters in OCD psychopathology have raised the possibility of clinical value of glutamate modulators in treating OCD (Pittenger et al., 2006; Grados et al., 2013). In support of this, the voltage-sensitive $\mathrm{Na}^{+}$ channel blocker that prevents glutamate release, riluzole (Pittenger et al., 2015), and the NMDA receptor antagonist, memantine (Modarresi et al., 2018), have shown benefit as augmentation strategies in patients with SSRI-refractory OCD. Apart from its role in GABAglutamate signaling, EAAT3 also mediates cysteine uptake necessary for neuronal glutathione (GSH) production, thereby regulating cellular redox status. This may explain the abundant evidence from both clinical and preclinical studies describing redox imbalances in OCD (for reviews, see Smith et al., 2016; Szechtman et al., 2017). Targeting these processes in treating OCD is discussed later (section III.B.1).

As reviewed earlier in section II.D, there are several forms of psychotherapy, including ERP, all of which emphasize that some level of learning takes place. Some investigators suggested that ERP is similar in structure to extinction learning (Lewin et al., 2014). This similarity raised the clinical question of whether ERP learning could be enhanced with a suitable pharmacological intervention, given basic science evidence that the NMDA partial agonist D-cycloserine can enhance extinction of learned fear (Davis et al., 2006). Early clinical reports did indeed observe that D-cycloserine augmented the effects of psychotherapy in the treatment of OCD (Chasson et al., 2010; Otto et al., 2010) in youths (Storch et al., 2010; Farrell et al., 2013) and adults (Wilhelm et al., 2008; Andersson et al., 2015), as well as in the treatment of non-OCD-related anxiety (Ressler et al., 2004; Hofmann et al., 2006; Guastella et al., 2008; Smits et al., 2013a,b). However, in a recent placebo-controlled randomized clinical trial, there was no evidence for added efficacy of D-cycloserine augmentation over CBT monotherapy, although the investigators encouraged trying different augmentation protocols to enhance outcome (Storch et al., 2016). Indeed, a recent meta-analysis of D-cycloserine augmentation of CBT in anxiety, OCD, and PTSD evaluated $>1000$ participants and found that there is a small statistically significant improvement with D-cycloserine augmentation at the end of treatment but, interestingly, not at the midtreatment time point (Mataix-Cols et al., 2017). This suggests that the augmentation effect of D-cycloserine is unlikely to result from an increase in the speed of learning during ERP.

In summary, some work has explored the possible clinical value of glutamate modulators in treatment strategies for OCD, but early encouraging findings have not been sustained in more recent trials. An interesting possibility is that rather than relating to a specific neurobiological deficit underlying OCD, glutamate modulation could have a more general effect in promoting corrective learning as part of psychotherapy.

\section{B. Probing the Role of Other Endogenous Processes in Obsessive-Compulsive Disorder}

Other research probes for faulty neurobiology in OCD by examining a variety of factors perturbing endogenous processes that affect the normal operation of the brain, as discussed next.

1. Oxidative Stress. Altered redox status may be relevant to understanding a number of neuropsychiatric disorders (Brand et al., 2015). The brain is particularly sensitive to the adverse effects of oxidative stress, due to its high oxygen consumption, low levels of antioxidants like GSH, and high levels of phospholipids that are easily damaged by free radicals. Previous studies suggested that OCD may be linked to changes in antioxidant status and increased lipid peroxidation (Kuloglu et al., 2002; Ersan et al., 2006; Selek et al., 2008; Ozdemir et al., 2009). Furthermore, it has been noted that symptom severity in patients with OCD may be positively correlated with the production of reactive nitrogen species (Atmaca et al., 2005) and products of lipid peroxidation (Chakraborty et al., 2009).

Considering work in animal models, Güldenpfennig et al. (2011) were the first to note disturbances in frontal-cortical GSH (reduced GSH and oxidized GSH disulfide) and its correlation with the severity of stereotypy in the deer mouse model of OCD. This finding suggests increased cycling and utilization of GSH, and strongly points to coupling between oxidative stress and symptom severity in this model and possibly in OCD, thus consistent with human studies (Atmaca et al., 2005; Chakraborty et al., 2009). Consistent with these findings, adjunctive $N$-acetyl cysteine (NAC), a GSH precursor and glutamate-NMDA receptor modulator (Berk et al., 2013), has been found to exert beneficial effects in OCD as a cotreatment with SRIs 
(Afshar et al., 2012; Paydary et al., 2016; Bhaskara, 2019). Recent systematic reviews find the available evidence of clinical benefit encouraging but at present weak (Oliver et al., 2015; Smith et al., 2016; Couto and Moreira, 2018). Subsequently, clinical studies have noted lower posterior cingulate cortex GSH levels in patients with OCD, indicative of increased oxidative stress secondary to hypermetabolism in this brain region (Brennan et al., 2016).

Considering these NAC findings, there is increasing interest in investigating nutraceuticals (natural products) for OCD, which have revealed encouraging activity in modulating key pathways suggested to be involved in the pathogenesis of OCD (e.g., glutamatergic and serotonergic pathways) (Camfield et al., 2011). Plantbased interventions with known active constituents that modulate these pathways include myo-inositol (MI), glycine, and milk thistle (Silybum marianum). The glucose isomer MI is a substance that has a long history of use in the treatment of psychiatric disorders and is discussed later in section III.B.2. The amino acid glycine is a nutrient that affects cortical glutamatergic function. Glycine and D-serine are agonists at NMDA receptors and have shown potential in ameliorating OCD in humans (Oliet and Mothet, 2009; Singer et al., 2010). The putative anti-OCD effects of milk thistle may be attributable to the flavonoid complex silymarin (Osuchowski et al., 2008), which may mediate an increase in cortical serotonin levels via inhibition of monoamine oxidase (Mazzio et al., 1998), and hence exert its antiobsessional effects in this way. An 8-week randomized double-blind clinical trial revealed equal efficacy in a head-to-head comparison between milk thistle and fluoxetine from baseline to endpoint in patients with OCD (Sayyah et al., 2010).

Curcumin and crocins are other antioxidants that warrant interest. Although no clinical studies have been undertaken, chronic oral administration of curcumin was found to significantly reduce compulsive checking and ritualistic behaviors in rodents and to reverse quinpirole-induced deteriorations in spatial learning (Chimakurthy and Murthy, 2010). These effects were found to be comparable to an SSRI. Curcumin also reversed quinpirole-induced increases in dopamine and increased 5-HT concentrations in the brain (Lopresti, 2017). Crocins too have been found to attenuate mCPPinduced excessive self-grooming in rats (Georgiadou et al., 2012).

Another important contributor to cellular redox is the reactive nitrogen intermediate, nitric oxide (NO), especially via its conversion under oxidative stress conditions to peroxynitite $\left(\mathrm{ONOO}^{-}\right)$(Oosthuizen et al., 2005). NO therefore has significant implication for redox dysfunction in OCD, as discussed below in section III.B. 2 .

In summary, altered redox status may compromise the operation of the brain as a whole and may thus affect the function of specialized systems, including those that may underlie OCD. Hence, even though evidence indicates that agents reducing oxidative stress may have a beneficial impact on OCD, a disordered redox system is not a neurobiological fault specific to OCD (see also Brand et al., 2015).

2. Second Messengers and Their Precursors. The human brain contains a high concentration of MI, an endogenous isomer of glucose; MI is an important dietary and cellular constituent and a key metabolic precursor in the phosphoinositide (PI) intracellular secondary messenger system (PI cycle) (Kim et al., 2005). The PI cycle is activated in neurons after ligand binding with $(\mathrm{Gq})$ protein-coupled receptors, including adrenergic $(\alpha 1 \mathrm{~A}$ and $\alpha 1 \mathrm{~B})$, serotonergic (5-HT1C and 5-HT2), dopaminergic (D1), glutamatergic (mGlu1 and mGlu5), and cholinergic (M1 and M3) receptor types (Fisher et al., 1992). Receptor activation produces the second messengers inositol 1,4,5-trisphosphate, which mobilizes intracellular $\mathrm{Ca}^{2+}$, and diacylglycerol, which activates protein kinase C (Harvey, 1997). Exogenous administration of MI can elevate MI in both cerebrospinal fluid and the brain (Einat and Belmaker, 2001), where it is stored predominantly in astrocytes (Frey et al., 1998).

The commonality of action of MI with known treatments of OCD (e.g., SSRIs) at the foregoing receptors suggests that a similar clinical response to MI may be expected and over the same treatment period (Levine et al., 1999; Einat and Belmaker, 2001). Marazziti et al. (2000, 2002) observed increased protein kinase $\mathrm{C}$ activity that decreases 5 -HT reuptake in OCD. MI may potentially correct hyperactivity in the PI pathway by means of downregulating its activity through altered gene expression (Harvey et al., 2002) and changes in 5-HT2 and D2 receptor density (Harvey et al., 2001). In preliminary clinical studies in patients with OCD, treatment with $18 \mathrm{~g} \mathrm{MI} /$ day for 6 or 12 weeks was found to be effective in reducing OC symptoms (Fux et al., 1996; Carey et al., 2004). Interestingly, the clinical response to MI was associated with reduced brain perfusion measured with single photon emission computerized tomography in the prefrontal cortex, temporal lobe, and parietal cortex (Carey et al., 2004). Using MI as augmentation therapy with SSRIs for OCD, however, has been disappointing (Fux et al., 1999; Seedat and Stein, 1999), although there is preliminary evidence that it may ameliorate symptoms of trichotillomania and compulsive skin picking (Seedat et al., 2001).

The NO cascade plays a central role in regulating the cGMP second-messenger cascade. cGMP is produced after NO-mediated activation of guanylate cyclase where it is involved in a host of neuromodulatory actions (Oosthuizen et al., 2005), including neurotransmitter release and synaptic plasticity. NO itself is a reactive nitrogen intermediate derived from the semi-essential amino acid, L-arginine, via either 
constitutive or inducible/immunologic NOS. The former is involved in the neurotransmitter and neuromodulator functions of NO, whereas the latter is involved in immune function, especially the cytotoxic actions of immune-activated macrophages via mediation of nitrosative stress (Oosthuizen et al., 2005). Metabotropic glutamate receptor mGluR5, $\alpha$-amino-3hydroxy-5-methyl-4-isoxazolepropionic acid, and NMDA receptors together especially play a role in modulating synaptic plasticity via effects on the NO-cGMP pathway (Boix et al., 2011).

Therefore, metabotropic glutamate receptors and ionotropic receptors, together with downstream messengers such as NO, are important mediators of synaptic plasticity and as such have been implicated in anxiety disorders (Harvey et al., 2004; Oosthuizen et al., 2005). Considering a possible involvement in OCD, inhibition of NO synthesis prevents excessive marble-burying behavior (Krass et al., 2010), a behavioral marker of compulsive behavior in rodents. In a related study, Umathe et al. (2009a) found that OC behaviors in mice appear related to increased levels of NO in brain, whereas the anticompulsive effects of paroxetine are related to the drug's ability to decrease brain levels of NO (Finkel et al., 1996; Wegener et al., 2003). Consistent with these results in animals, studies in patients with OCD have shown significantly elevated plasma nitrate levels $\left(\mathrm{NO}_{3}^{-}\right)$, a stable marker of $\mathrm{NO}$, that were positively correlated with YBOCS scores (Atmaca et al., 2005). Similarly, Yilmaz et al. (2016) investigated variations in the levels of $\mathrm{NO}$, asymmetric dimethyl arginine, symmetric dimethyl arginine, and L-arginine levels in patients with OCD. The investigators found that although NO levels were increased, albeit narrowly missing statistical significance, patients with OCD had significantly lower levels of asymmetric dimethyl arginine, symmetric dimethyl arginine, and L-arginine compared with the controls (Yilmaz et al., 2016).

Precursors of NO synthesis, such as L-arginine as well as NOS and guanylyl cyclase inhibitors, may therefore have modulatory and/or therapeutic value in OC states. In this regard, agmatine, a polyamine widely distributed in the mammalian brain that interacts with nitrergic systems as well as imidazoline receptors, has attracted interest in the treatment of a number of neuropsychiatric and neurologic disorders (Laube and Bernstein, 2017; Neis et al., 2017). Agmatine significantly inhibited compulsive marble-burying behavior in mice, whereas the NO precursor, L-arginine, increased compulsive burying behavior as well as brain nitrite $\left(\mathrm{NO}_{3}^{-}\right)$levels. Similarly, treatment with various neuronal NOS inhibitors attenuated such behavior as well as brain $\mathrm{NO}_{3}^{-}$levels (Gawali et al., 2016). Behavioral response to agmatine was exacerbated by prior administration of L-arginine $(400 \mathrm{mg} / \mathrm{kg})$ and abrogated by NOS inhibitors, with hippocampal and cortical changes in $\mathrm{NO}_{3}^{-}$(Gawali et al., 2016). Since $\mathrm{NO}$ is a reactive nitrogen intermediate, elevated levels of $\mathrm{NO}$ and the subsequent formation of peroxynitrite $\left(\mathrm{ONOO}^{-}\right)$in the presence of superoxide $\left(\mathrm{O}_{2}^{-}\right)$under high levels of oxidative stress will contribute to the redox environment of the cell. Therefore, the NO cascade has implications for redox functioning for the brain as a whole, with effects on OCD (see section III.B.1).

Another second messenger of possible relevance to OCD is cAMP, which mediates intracellular signal transduction in the cAMP-dependent pathway. cAMP is synthesized from ATP by adenylate cyclase (AC) located on the inner side of the plasma membrane, which in turn is activated by a range of signaling molecules through the activation of AC stimulatory $\mathrm{G}(\mathrm{Gs})$ protein-coupled receptors or AC inhibitory $\mathrm{G}(\mathrm{Gi})$ protein-coupled receptors (Katzung, 2017). cAMP decomposition into $5^{\prime}$-AMP is catalyzed by the enzyme phosphodiesterase (PDE); there are a number of PDE isoforms, each displaying unique functions with respect to the breakdown of cyclic nucleotides, such as cAMP, cGMP, and cyclic inosine monophosphate (Houslay and Milligan, 1997; Houslay et al., 1998). Of relevance, established OCD-related pharmacotherapies, such as SSRIs and neuroleptics, all have effects on the ACcAMP pathway through inhibition or stimulation of AC (Katzung, 2017), and such activity may in part mediate the time-course profile of the drugs' therapeutic effect.

Looking more specifically at cAMP signaling in OCD and in animal studies, severity of stereotypy in deer mice is associated with elevated frontal-cortical cAMP and reduced PDE4 activity; moreover, in high stereotypic animals, chronic fluoxetine reduced both stereotypy and cortical cAMP and PDE4 activity (Korff et al., 2009). Furthermore, elevated cAMP in high stereotypic mice could be related to increased 5-HT1A-AC-cAMP signaling with reduced hydrolysis by PDE4 (Korff et al., 2009). That 5-HT1A agonists reduce stereotypy (Korff et al., 2008; Tucci et al., 2013, 2014b), and 5-HT1A receptor activation promotes $\mathrm{AC}$ sensitization (Hensler et al., 1996), supports a role for AC-cAMP coupling in OCD (Perez et al., 2000; Marazziti et al., 2001, 2009) and its response to treatment. Indeed, clinically effective OCD treatments prevent 5-HT1B receptor-induced repetitive behavior and striatal activation (Ho et al., 2016).

cAMP response element-binding protein (CREB) is a cellular transcription factor that binds to certain DNA sequences called cAMP response elements, thereby increasing or decreasing the transcription of specific genes. CREB plays a crucial role in memory (Benito and Barco, 2010), learning (Wen et al., 2015), and neuroinflammation (Krasnova et al., 2016). PDE4 is involved in regulating glial inflammatory reactions (Beardsley and Hauser, 2014) and regulating the reinforcement (addictive) effects of illicit substances. Simply put, cAMP causes the phosphorylation of CREB (Chiu et al., 2014) that in turn regulates 
neuroinflammation and neuroplasticity ( $\mathrm{Li}$ et al., 2018), whereas PDE4 catalyzes the degradation of cAMP to biologically inactive 5'-AMP. CREB-mediated gene expression plays a critical role in memory through synaptic plasticity (Benito and Barco, 2010), leading to functional, structural, and neuroplastic alterations in memory- and reward-related brain areas (Dreyer, 2010). Inhibition of PDE4, with agents like rolipram and ibudilast, upregulates cAMP expression and modulates neuroinflammation (Li et al., 2018). Interestingly, preclinical studies highlight a dysfunctional cAMP-PDE4 system in high stereotypic deer mice (Korff et al., 2009) and this, together with the finding that rolipram decreases methamphetamineinduced stereotypy (Iyo et al., 1996), raises the possibility that PDE4 active compounds may represent novel treatment options for OCD.

3. Microbiome/Probiotics. The brain-gut-microbiota axis is a complex bidirectional communication system that integrates neural, hormonal, and immunologic signaling between the gut and brain via a complex network involving the central, autonomic, and enteric nervous systems. Although evidence is more profound in preclinical models, the brain-gut-microbiota axis seems to play a contributory role in mental illness and is particularly relevant in abdominal pain syndromes and emotional regulation (Turna et al., 2016).

The bacteria that make up the human gut microbiome outnumber human cells 10-fold and aid in facilitating nutrient absorption by metabolizing indigestible dietary compounds, developing gut-associated lymphoid tissue, producing vitamins, and preventing colonization by pathogens (Turna et al., 2016). The primary gut microbiome inhabitants include Bacteroidetes and Firmicutes, Proteobacteria, Actinobacteria, Fusobacteria, and Verrucomicrobia phyla (Gill et al., 2006). Although the gut flora is largely stable in adult life, it can be modulated by infection, antibiotics, and probiotics, and its dysfunction may lead to states of chronic and acute disease. A number of illnesses, such as inflammatory bowel disease, autism spectrum disorder (ASD), celiac disease, diabetes, and obesity, are capable of changing the gut microbiome. The gut-brain relationship has more recently attracted significant interest with respect to a possible role in psychiatric illnesses, including anxiety and mood disorders (Turna et al., 2016). Such a role is plausible, given the ability of the gut microbiome to modify neurotransmitter and gut hormone release, activation of enteric nervous systembrain signaling via ascending neural pathways, and activation of the immune system via cytokine release (Turna et al., 2016). With psychiatric illness so ensconced in monoaminergic and other neurotransmitter dysfunctions, that microbiota can alter these transmitters is noteworthy. Thus, the microbiota has effects on serotonin (Candida, Streptococcus, Escherichia, Enterococcus), dopamine (Bacillus, Serratia), GABA (Lactobacillus, Bifidobacterium), norepinephrine (Escherichia,
Bacillus, Saccharomyces), and acetylcholine (Lactobacillus) (Collins et al., 2012). Strains of Lactobacillus and Bifidobacterium produce GABA (Collins et al., 2012), whereas oral administration of Bifidobacterium infantis increases levels of the serotonin precursor, tryptophan (Desbonnet et al., 2008). As noted previously, both GABA and serotonin are implicated in the neurobiology of OCD.

With animal studies noting that anxiety disorders are an acquired early-life neurodevelopmental anomaly (Regenass et al., 2018), it is noteworthy that anxiety manifestations cannot necessarily be normalized by altering the microbiota in adulthood (Diaz Heijtz et al., 2011; Neufeld et al., 2011a,b; Desbonnet et al., 2015). Importantly, depletion of the gut microbiota is associated with significant changes in neuromodulatory and neuroinflammatory substances known to have neurobehavioral effects, such as the tryptophan-kynurenine or tryptophan catabolic (TRYCAT) pathway (Desbonnet et al., 2015). The latter pathway is closely linked to immune activation by proinflammatory cytokines, and TRYCAT may have a role in the pathogenesis of psychiatric illness by diverting tryptophan metabolism away from serotonin synthesis that in turn may underlie anxiety and depressive symptoms (Desbonnet et al., 2015; Möller et al., 2015). The pathway can also affect neuroplasticity by altering the ratio between quinolinic acid and kynurenic acid, which has been termed the neuroprotective index (Möller et al., 2015).

Preliminary studies in psychiatric populations support gut dysbiosis in some conditions, with preclinical studies supporting the view that probiotics have therapeutic benefits (Dinan and Cryan, 2017). That probiotics could alter the course of neuropsychiatric disease is suggested in a recent clinical study describing how probiotics alter brain activity as assessed by functional magnetic resonance imaging (Allen et al., 2016). This study also shows how Bifidobacterium longum alters brain electrical activity, whereas Dolan et al. (2016) describe how the introduction of Bifidobacterium and Lactobacillus into treatment improves symptoms of anxiety, depression, ASD, OCD, and memory abilities.

Direct evidence for the involvement of the microbiota, or modification with probiotics, in OCD(Skarphedinsson et al., 2015) is lacking. Nevertheless, a study in healthy humans found that $L$. helveticus and B. longum administered daily for 30 days reduced OC subscores (Messaoudi et al., 2011). Translational findings in animal models are also limited. Chronic treatment with L. rhamnosus reversed 5 -HT1A/1B receptor agonist-induced marble-burying and locomotor behavior (OCD-like behaviors) in mice, and in this regard was found to be equivalent to chronic fluoxetine treatment (Kantak et al., 2014). In the QS rat model of OCD, changes in several communities of gut bacteria (predominantly Lachnospiraceae and Ruminococcaceae) were associated with the induction of compulsive checking behavior; the researchers suggested 
that changes in these microbes, rather than directly producing OCD, may serve to support the energy use requirements of compulsive checking and OCD (Jung et al., 2018). Finally, work with the deer mouse model has described the microbial composition of mice that build excessively large nests (Scheepers et al., 2019), the latter an expression of compulsivity (Wolmarans et al., 2016). The authors found significant differences in gut microbiota in normal versus large nest builders, driven by a clustering of Prevotella and Anaeroplasma in normal nest builders compared to a clustering of Desulfovermiculus, Aestuariispira, Peptococcus and Holdemanella in the large nest building cohort. Importantly, these clusterings diverge on the basis of their role in inflammatory processes, where the genera associated with normal nest builders has been shown to have anti-inflammatory properties, while the opposite has been shown with respect to the genera associated with the large nest builders (Scheepers et al., 2019).

Studies in Gille de la Tourette syndrome (TS) may be instructive as to the role of microbiota in OCD. TS is a neurodevelopmental disorder characterized by recurring motor and phonic tics, with its pathogenesis thought to reflect dysregulations in dopamine signaling and other neurotransmitters leading to excitation/ inhibition imbalances. TS may present with motor stereotypies that resemble those observed in OCD. A recent case study described a cause-and-effect relationship between Staphylococcus aureus colonization and tic improvement in a patient with TS, as well as between bacterial decolonization and tic exacerbation (Eftimiadi et al., 2016). Clinical improvements in motor tic frequency and severity were observed during the $S$. aureus colonization phase, which were correlated with the downregulation of antistreptococcal and anti-D1/D2 dopamine receptor antibody production. Although limited in scope, these findings do lend support to the notion that dysregulation of the microbiome may be involved in OCD.

In summary, it has been proposed that OCD may be related to changes in communication between the microbiome, inflammatory response, and hypothalamic-pituitaryadrenal (HPA) axis (Turna et al., 2016). However, these associations may not be pointing to an underlying neurobiological fault specific to OCD; rather, the microbiome may have a more general effect on the operation of the brain as a whole, thus altering the functioning of specialized systems, including those underlying OCD.

4. Neurosteroids and Neuropeptides. Various neuropeptides have attracted interest with respect to the pathology and treatment of OCD, including oxytocin, arginine vasopressin, adrenocorticotropic hormone (ACTH), corticotropin-releasing factor, and neurosteroids such as somatostatin and opioids (McDougle et al., 1999). There are extensive functional interactions between neuropeptidergic and monoaminergic systems in the brain, and these systems are often neuroanatomically colocalized.
Neuroactive steroids not only act as transcriptional factors in the regulation of gene expression, but they also modify neuronal excitability and play an important role in the pathophysiology of mood and anxiety disorders. $3 \alpha-$ reduced neuroactive steroids especially act as positive allosteric modulators of the $\mathrm{GABA}_{\mathrm{A}}$ and glutamateNMDA receptors. These steroids include allopregnanolone or allotetrahydrodeoxycorticosterone, progesterone, dehydroepiandrosterone, and dehydroepiandrosterone 3-sulfate (Umathe et al., 2009b; Schüle et al., 2011). Dysregulation of neurosteroids has been identified in patients with OCD (Bigos et al., 2009), with animal studies indicating that neurosteroids can modulate OC behavior in a bidirectional manner (Umathe et al., $2009 \mathrm{~b})$. Considering the previously reviewed role of dopamine, 5-HT, and GABA-glutamate in OCD, it is noteworthy that allopregnanolone and dehydroepiandrosterone/dehydroepiandrosterone 3-sulfate have opposite effects on $\mathrm{GABA}_{\mathrm{A}}$ and NMDA receptors (Compagnone and Mellon, 2000), and that neurosteroids modulate dopaminergic transmission through $\mathrm{GABA}_{\mathrm{A}}$ receptors (Jaworska-Feil et al., 1998) and increase firing of raphe nuclei serotonergic neurons (Robichaud and Debonnel, 2006). Moreover, fluoxetine increases levels of allopregnanolone in the brain (Pinna et al., 2006).

The neuropeptides oxytocin and prolactin are implicated in promoting normal maternal care and aggression, as well as in decreasing anxiety and depression in humans (Groër, 2005; Skrundz et al., 2011) and animal studies (Neumann et al., 2000; Champagne et al., 2001; Torner et al., 2002; Bosch et al., 2005; Salais-López et al., 2017). Oxytocin is recognized in regulating anxietylike (Neumann and Slattery, 2016) and depression-like (Cochran et al., 2013) behaviors. Some studies have linked higher central (Leckman et al., 1994) and peripheral (Humble et al., 2013) oxytocin levels to OCD, whereas others have found no significant correlation with endogenous oxytocin levels (Altemus et al., 1999) or effect on OCD symptomology after exogenous administration of oxytocin (den Boer and Westenberg, 1992; Epperson et al., 1996). Interactions between oxytocin and the central and peripheral serotonergic systems (Jury et al., 2015), such as serotonergic-mediated modulation of prolactin secretion (Balsa et al., 1998; Chaiseha et al., 2010), might underlie these responses. However, plasma prolactin levels in patients with OCD after challenge with mCPP have been contradictory (den Boer and Westenberg, 1992; Leckman et al., 1994; Epperson et al., 1996; Altemus et al., 1999; Khanna et al., 2001; Humble et al., 2013), and further study as to the role of this neuropeptide in OCD is warranted. It has been suggested that oxytocin might enhance the sensitivity of neural circuits that detect specific categories of threat (e.g., threats to the stability of a pair bond, or threats to one's offspring), and activate adaptive motivational systems and behaviors to respond to these potential threats (Leckman et al., 1999; Feygin et al., 2006). 
In summary, although neurosteroids and neuropeptides may have effects on OCD, the reviewed work does not point to an underlying neurobiological fault specific to OCD. Instead, their mode of action is likely more general, affecting the functioning of brain processes as a whole.

5. Neurotrophic Factors. A recent meta-analysis found that brain-derived neurotrophic factor (BDNF) is significantly reduced in individuals with OCD (Suliman et al., 2013). A significant association was detected between BDNF polymorphism rs2883187 and OCD (Abdolhosseinzadeh et al., 2019), and Hall et al. (2003) showed a strong association between BDNF gene sequence variants, including the Val66Met variation, and OCD. Subsequent studies also showed that the Val66Met BDNF gene variant is a risk allele for development of OCD, possibly in a sex-specific manner (Hemmings et al., 2008; Katerberg et al., 2009). However, research exploring a possible pathophysiological role for BDNF in OCD is lacking (Oliveira-Maia and Castro-Rodrigues, 2015). Interestingly, a recent study by Şimşek et al. (2016) looking at serum BDNF, ACTH, and cortisol levels in treatment-naïve children with OCD suggests that BDNF levels adaptively increase as a result of the damaging effects of HPA axis hyperactivity on brain tissue in the early stages of OCD, arguing that HPA axis abnormalities and BDNF may play a role in the pathogenesis of the illness.

Considering the critical role for 5-HT in the neurobiology of OCD and in its treatment, animal studies with SSRIs demonstrate that 5-HT shapes neuronal networks during development, so that deficiencies affect the pathophysiology and long-term outcome of a brain disorder like OCD (Kraus et al., 2017). SSRIs might reactivate serotonin's ability to mediate developmental plasticity (Maya Vetencourt et al., 2008; Castrén and Rantamäki, 2010) via close molecular connections between serotonergic receptors with BDNF and with intracellular signaling cascades, leading to cytoskeletal rearrangement (Mattson et al., 2004; Rantamäki and Castrén, 2008). For example, serotonin modulates glutamatergic transmission that in turn mediates NMDA receptor-dependent plasticity (Bennett, 2010; Sanacora et al., 2012). Moreover, 5-HT is linked to cell adhesion molecules (Lesch and Waider, 2012), which are part of the extracellular matrix and crucial for developmental plasticity (Varea et al., 2007). Given that anxiety and mood disorders are often comorbid with OCD, environmental effects might be a crucial modulating factor for serotonergic neuroplasticity, in which stress and negative life events will profoundly affect SSRI-mediated neuroplasticity (Wu et al., 2014; Alboni et al., 2017). Indeed, such comorbidities and resistance to neuroplastic change may be part of the mechanism for the time delay in response to SSRIs that is typically seen in patients with OCD, as mentioned earlier.

\section{Probing the Neuroanatomical Location of the Neurobiological Fault in Obsessive- Compulsive Disorder}

Various findings have suggested there to be an identifiable neurologic basis for OCD. First, the symptoms of OCD often present together with several neurologic disorders, such as postencephalitis parkinsonian syndrome (Schilder, 1938), Sydenham chorea (Swedo, 1989), bilateral necrosis of the globus pallidus (Laplane et al., 1989), Huntington chorea (Cummings and Cunningham, 1992), and TS (Pauls et al., 1986), suggesting that OCD may have a genetic and/or structural basis related to these disorders.

Second, positron emission tomography studies showed that changes in glucose activity in the OFC and caudate nucleus correlate with the presence and disappearance of OCD symptoms (Benkelfat et al., 1990; Baxter et al., 1992; Swedo et al., 1992). Other neuroimaging studies also implicated the OFC in OCD (McGuire et al., 1994; Rauch et al., 1994; Saxena et al., 1998; Adler et al., 2000; Kim et al., 2001).

Third, as reviewed in section II.B, pharmacological treatments that targeted specific neurotransmitter systems could alleviate OCD symptoms. Fourth, psychosurgery of particular anatomic regions (section II.C.1) could improve severe cases of OCD, in particular anterior capsulotomy or cingulotomy (Kettl and Marks, 1986; Chiocca and Martuza, 1990; Jenike et al., 1991; Baer et al., 1995; Dougherty et al., 2002). Finally, a susceptibility marker predisposing some individuals to develop OCD has been identified-namely, the D8/17 antigen on the surface of peripheral blood mononuclear cells. The postulated mechanism by which D8/17-positive individuals could develop OCD involved an immune response to group A $\beta$-hemolytic streptococcal infection and the production of antibodies that crossreacted with basal ganglia antigens, damaging these neurons (Swedo et al., 1997).

Consistent with the foregoing observations, the first proposals for a neuroanatomy of OCD assigned a central position to the basal ganglia (Wise and Rapoport, 1989; Baxter, 1992; Insel, 1992). Originally, an appreciation for the importance of the basal ganglia in OCD stemmed from insights that the basal ganglia may be a repository of innate motor programs (MacLean, 1978) and that OCD rituals may be examples of such species-typical programs (Swedo, 1989; Rapoport and Fiske, 1998). Drawing on a conceptual organization of the basal ganglia as the nodal point of converging but segregated closed-loop circuits involving cortex-basal ganglia-thalamus-cortex pathways (Alexander et al., 1986), Wise and Rapoport (1989) proposed that pathologic activity in such CSTC circuits would produce reverberating activity and result in a persistent discharge of the innate motor programs characteristic of OCD behavior. Subsequent proposals for a neuroanatomical model of 
OCD maintained this conceptual schema of CSTC loops, but highlighted the importance of the OFC as a nodal point and provided more elaborate details of the circuit diagram and how abnormalities in the CSTC circuits would produce the OCD pathophysiology (Modell et al., 1989; Baxter, 1990; Insel, 1992; Saxena et al., 1998; Saxena and Rauch, 2000; Aouizerate et al., 2004; Friedlander and Desrocher, 2006; Huey et al., 2008).

Although consistent with the CSTC circuits model of OCD, further research suggested the need to incorporate connections with additional brain regions, such as the amygdala, hippocampus, anterior cingulate, prefrontal cortex, and parietal cortex (Menzies et al., 2008; Milad and Rauch, 2012; Nakao et al., 2014). These elaborations of the neuroanatomical OCD model stemmed from functional brain imaging studies revealing a difference between patients with OCD and controls during cognitive and affective tasks in these regions (e.g., Mataix-Cols et al., 2004; Simon et al., 2010, 2014; Via et al., 2014; Norman et al., 2016). Moreover, even at rest, statistically significant structural and functional regional differences were also found (e.g., Rotge et al., 2009; de Wit et al., 2014; Göttlich et al., 2014; Piras et al., 2015; Reess et al., 2016; Tian et al., 2016; Boedhoe et al., 2017, 2018; Fouche et al., 2017; Gürsel et al., 2018; Koh et al., 2018; Brennan et al., 2019; Hao et al., 2019; Yang et al., 2019).

However, despite the heuristic value of the proposed neuroanatomic models, the site of the neurobiological fault has not been identified, nor is it even known whether the neurobiological fault producing OCD symptoms is necessarily a constituent of the proposed circuit models of OCD. This is because human brain imaging studies are predominantly correlational in nature and are thus inadequate to establish a cause-and-effect relationship. For instance, although OFC overactivity noted earlier is generally interpreted as indicating pathology, Zald and Kim (2001) cautioned that it may be an effect rather than the cause of OCD. They point out that in both patients with OCD and control subjects, activity in the OFC is a normal correlate of silent ruminative thinking (Cottraux et al., 1996), and thus "increased resting OFC metabolism in OCD [may simply reflect] a greater engagement of obsessive ruminations in OCD patients" (Zald and Kim, 2001, p. 61). Similarly, smaller or greater values in the parameters of a brain structure compared with controls may not necessarily be an indication of a brain "abnormality" in OCD. Rather, it may indicate an acquired "expertise" in performing OCD-related tasks, just like London taxi drivers show marked structural changes in the hippocampus induced through their experience of navigating in a large complex city (Maguire et al., 2006).

To test the proposed circuit models of OCD, research in animals is needed in which the independent variable is the experimental alteration of activity in a particular neural region and the dependent variable is performance of OCD-related behaviors (Szechtman and Eilam, 2005). A number of such studies have indeed shown that experimental perturbation of neural activity in several regions of the CTSC circuit (OFC, nucleus accumbens, anterior cingulate, striatum, STN, amygdala) does produce effects on the display of OCD-like behaviors (e.g., Campbell et al., 1999; Winter et al., 2008; Klavir et al., 2009; Dvorkin et al., 2010; Yang and Lu, 2011; Ahmari et al., 2013; Ballester González et al., 2015; Burton, 2017; Sun et al., 2019), consistent with current neuroanatomical models of OCD. However, although these studies implicate activity in those brain regions as important for the display of OCDlike behaviors, they do not identify the mechanism of the OCD pathogenesis or whether the neurobiological fault producing OCD is confined to any one specific neuroanatomical site.

In summary, there is a fairly extensive body of literature showing that the neuroanatomical substrate underpinning the presentation of OCD involves CSTC circuits as well as frontolimbic and frontoparietal regions. However, although the empirical findings implicating the involvement of these regions in OCD show that some regional property is different compared with controls, such data, in and of themselves, do not constitute evidence of an "abnormality" - that this difference is the neurobiological fault underlying OCD. A good theory of OCD will need to take account of these findings but embed them into a conception of what normal functions the underlying brain system subserves and advance a more specific hypothesis about the regulatory fault by which this normal system yields the abnormal phenomena of OCD. In section IV, we take a novel approach to propose a theory of OCD that is more specific about the nature of the underlying regulatory fault, outline the normal brain system that is affected by this fault, and then embed the foregoing findings into this theory as its hypothesized neural basis.

\section{Probing Etiology for the Neurobiological Fault in Obsessive-Compulsive Disorder}

Etiological research looks for factors associated with the occurrence of OCD, which may provide important clues about an underlying neurobiological fault. The following section covers a spectrum of factors of possible etiological significance in OCD, including genetics, age of onset, the hormonal system, infection and the immune system, and neuroinflammation.

1. Genetics of Obsessive-Compulsive Disorder.

a. Human Studies of the Genetic Correlates of Obsessive-Compulsive Disorder. Over the past decades, numerous twin and family studies have been conducted with the aim of determining heritability estimates and identifying candidate genes that may contribute to the etiopathology of OCD (Stewart et al., 2007a,b; Walitza et al., 2010; Kelmendi et al., 2011; Sampaio et al., 2011, 2015; Bandelow et al., 2016). In addition, candidate gene and genetic linkage studies 
have been conducted that placed a major focus on genes involved in serotonergic, dopaminergic, and glutamatergic signaling (for reviews, see Pauls et al., 2014; Sinopoli et al., 2017). However, due to several factors, including small sample sizes and differences in the selection criteria of participants, collective findings from these studies are inconclusive. More recently, research has focused on large genome-wide association studies (GWASs). Instead of looking at specific OCD theory-based genes (candidate gene studies) or aiming to identify cotransmission of specific DNA markers of OCD within families (linkage studies), GWASs attempt to identify population associations between DNA markers and OCD (Pauls, 2010).

Although previous studies demonstrated associations between OCD and polymorphisms in genes that play a role in serotonergic (Denys et al., 2006) and dopaminergic (Nicolini et al., 1996) neurotransmission, a recent GWAS comprising 2688 OCD cases and 7037 matched controls (Arnold et al., 2018) failed to demonstrate any such associations. However, although no single-nucleotide polymorphism reached genome-wide significance in that study, two genes involved in glutamatergic neurotransmission, namely, GRID2 (Glutamate Ionotropic Receptor Delta Type Subunit 2) and DLGAP1 (DLG Associated Protein/Guanylate Kinase-Associated Protein), were identified as possible genetic correlates of OCD. Furthermore, these findings are in line with two previous GWASs that also identified genes of the glutamatergic system in OCD heritability, such as GRIN2B (Glutamate Ionotropic Receptor NMDA Type Subunit 2B) (Arnold et al., 2009) and SLC1A1 (Stewart et al., 2007a). These findings have reinforced interest in the clinical value of glutamate modulators in treating OCD (Pittenger et al., 2006; Grados et al., 2013).

Considering the high degree of comorbidity between OCD and other neuropsychiatric conditions, such as ASD, TS, tic disorder, and generalized anxiety disorder, it is interesting that none of these disorders demonstrate genetic overlap with OCD in terms of the foregoing specific genes (Yu et al., 2015a; Guo et al., 2017; O'Connell et al., 2018). Rather, it is possible that clinical overlap of OCD with other conditions is related to a distinct set of genetic polymorphisms; for example, TUBB3 (Tubulin Beta 3 Class III) has been associated with comorbid OCD and ASD (Guo et al., 2017). Finally, the polymorphisms relevant to OCD are likely to involve complex interactions, rather than straightforward additive effects, and more sophisticated analyses will be required to tease these out.

b. Animal Models Based on Gene Association Findings for Obsessive-Compulsive Disorder. As just summarized, the strongest candidate gene association findings in OCD identify genes related to glutamatergic neurotransmission—namely, SLC1A1, GRIN2B, GRID2, and $D L G A P 1$. The possible effects of these and other genes on OCD-like behavior have been explored using geneknockout mice models, as follows.

$i$. Solute Carrier Family 1 Member 1/Sodium-Dependent Glutamate/Aspartate Transporter 3 Null Mice. Unexpectedly, the behavioral assessment of Slc1a1 null mice does not suggest compulsive-like behavior (Zike et al., 2017a). Nonetheless, Slc1a1 mRNA and its corresponding protein, EAAT3, are strongly expressed in brain regions implicated in OCD (Nieoullon et al., 2006). Moreover, EAAT3 is also involved in mediating neuronal cysteine transport, an essential rate-limiting step in the synthesis of the antioxidant GSH (Aoyama et al., 2006; Aoyama and Nakaki, 2013). Hence, this model may suggest that the SLC1A1 gene contributes to glutamate-redox function, which may have some nonspecific bearing on OCD.

ii. Sap90/Postsynaptic Density Protein-95-Associated Protein 3 Null Mice. Sap90/postsynaptic density protein-95-associated protein 3 (Sapap3) null mice show compulsive-like grooming and abnormalities in glutamatergic neurotransmission and function (Welch et al., 2007; Burguière et al., 2013; Wan et al., 2014; Corbit et al., 2019). In OCD and trichotillomania populations, rare amino acid variants in DLGAP3, the human ortholog of Sapap3, have been identified. Another association study found nominally significant association with a number of common polymorphisms and haplotypes in individuals with a grooming disorder (trichotillomania, excoriation, or nail biting) in addition to OCD, but not in OCD alone (Bienvenu et al., 2009). However, as mentioned earlier (in section III.D.1.a), the largest GWAS in OCD instead found evidence of association with another DLGAP family member, DLGAP1.

SAPAP3 is a postsynaptic scaffolding protein that interacts with the postsynaptic density protein 95 and Shank protein families (Takeuchi et al., 1997) and is highly expressed within glutamatergic synapses of the striatum. Hence, the DLGAP family may be associated with the mechanics of neural transmission and thus, although not specific to OCD, may be involved more generally in the regulation of repetitive behavior, as suggested by Burguière et al. (2013).

A few other genes have been explored similarly (Campbell et al., 1999; Wang et al., 2009; Monteiro and Feng, 2016; Zike et al., 2017a), including HOXB8 (Greer and Capecchi, 2002), which is discussed later in section III.D.4. More generally, in the gene-knockout animal models, the focus tends to be on whether the resulting behavior patterns appear to serve as a "good model" of OCD symptoms. Instead, we would propose that these models be used in a somewhat different way, namely to illuminate what processes relevant to OCD are affected. For example, are these animals more sensitive to particular cues, or is their response more vigorous or do they perseverate such that their response is more persistent over time? 
c. Conclusion. In summary, the genetic data indicate that OCD is not the result of a singular genetic profile. Hence, the evidence suggests that there is no specific genetic anomaly for an underlying neurobiological fault in OCD. This does not rule out the possibility that there are interactions between various genetic, neurobiological, and environmental factors to produce OCD (Pauls et al., 2014).

2. Age of Onset. OCD can begin quite early in life. Diagnoses of childhood onset are made from as young as age 2 years, peaking at age $10-12$ years (Tanidir et al., 2015). Childhood-onset OCD has been proposed to have certain phenomenological characteristics that distinguish it from adult-onset OCD (Geller et al., 1998, 2001; Rosenberg and Keshavan, 1998). Apart from affecting more boys than girls, childhood-onset OCD is often less episodic, is more familial, and demonstrates a higher degree of comorbidity with tic disorders and ADHD (Geller et al., 1996; Masi et al., 2010; Sheppard et al., 2010), especially in male patients. Although male patients typically present with OCD at a younger age (Fontenelle et al., 2006; Mathis et al., 2011), the majority of adult-onset OCD cases are women (Dell'Osso et al., 2016). A biologic basis for the age effect remains poorly explored. Nevertheless, it is intuitive that perhaps ontogeny of certain neurotransmitter systems during development (e.g., dopamine, serotonin, noradrenaline; Frederick and Stanwood, 2009), or the presence of a dysfunctional microbiome (section III.B.3), and their interaction with the environment may influence the age of onset.

3. Hormonal Correlates of Obsessive-Compulsive Disorder. Women may have a higher risk for developing anxiety disorders, or exacerbation of their recent symptoms, during times of significant hormonal changes such as puberty, pregnancy, postpartum, and menopause (Pigott, 2003; Ross and McLean, 2006; van Veen et al., 2009; Hickey et al., 2012). In some studies, a considerable proportion (approximately 13\%-22\%) of female patients retrospectively associated the onset of their OC symptoms with the onset of menarche (Alpak and Karamustafalioglu, 2009; Guglielmi et al., 2014).

Several studies involving clinical subjects have also associated hormonal changes during the menstrual cycle with increases in OC symptoms. Approximately $40 \%$ of women with an OCD diagnosis report an exacerbation of symptoms during the premenstrual period (Lochner et al., 2005; Vulink et al., 2006; Guglielmi et al., 2014). Some studies have shown that OC symptoms can be ameliorated by blockade of gonadal steroids (Casas et al., 1986; Chouinard et al., 1996; Altemus, 2006).

One mechanism by which gonadal hormones might exacerbate anxiety and $\mathrm{OC}$ symptoms might be through altering serotonin neurotransmission. Consistent with this proposal, OCD and premenstrual dysphoric disorder are distinguished by their preferential response to
SSRIs (Eriksson et al., 1995; Greist et al., 1995; Freeman et al., 1999). Some evidence suggests that postpartum depression, which can have prominent OCD features, responds better to SSRI antidepressants (Altemus, 2006), although there is not enough evidence to support a clear superiority of SSRIs over psychotherapy or nortriptyline, particularly after the acute phase (De Crescenzo et al., 2014).

A number of retrospective surveys suggest that in women, pregnancy and childbirth might be associated with the exacerbation of existing clinical OCD symptoms as well as with an increased risk for OCD onset. Guglielmi et al. (2014) reported that in a large sample of women diagnosed with OCD, approximately $10 \%$ reported that the onset of their symptoms was associated either with pregnancy or the postpartum period (up to 1 year after delivery). Approximately 30\%-50\% of women in this same study reported an exacerbation of existing symptoms during pregnancy or the postpartum period, thereby ascribing causal significance to cyclical sex hormones. Moreover, those women who reported an exacerbation of OCD symptoms associated with their first pregnancy or delivery were significantly more likely to experience symptom exacerbation at subsequent pregnancies and deliveries, thus suggesting that hormonal factors associated with pregnancy and childbirth might be important triggers in at least a subgroup of women. This possibility is strengthened by the results of Forray et al. (2010), which indicated that women who reported a pregnancy- or perinatal-associated exacerbation of symptoms were more likely to also report a worsening of symptoms during the premenstruum. Unfortunately, however, prospective studies of this phenomenon are rare. The results of one of the few such studies are consistent with a postpartum increase in severity of OC symptoms compared with third-trimester levels, although the sample size was small (Chaudron and Nirodi, 2010). Nonetheless, a second prospective study of 56 primiparous women previously diagnosed with OCD failed to demonstrate significant changes in the severity of OCD symptoms across pregnancy and the postnatal period (House et al., 2016). Moreover, analyses of the relative risk of an OCD diagnosis associated with pregnancy or the postpartum period revealed only a small, nonsignificant increase compared with that of the general population (McGuinness et al., 2011).

Up to $37 \%$ of patients describing the onset of OCD symptoms noted that these symptoms were preceded by stressful life events (Real et al., 2011). Numerous investigations indicate positive associations between stress and the onset of OC symptoms (Toro et al., 1992; Findley et al., 2003; Rosso et al., 2012). In light of this apparent linkage between stress and OCD, researchers have looked for hyperactivity of the HPA axis functioning in OCD. Indeed, elevated HPA activity in OCD has been found by measuring cortisol at multiple points throughout the day or by 24-hour urinary free cortisol 
collection (Kluge et al., 2007; Gustafsson et al., 2008). Similarly, elevated cerebrospinal fluid levels of corticotropinreleasing factor and ACTH have also been reported in OCD compared with healthy controls (Altemus et al., 1992; Catapano et al., 1992; Monteleone et al., 1997; Kluge et al., 2007). Moreover, nonsuppression of cortisol secretion in the dexamethasone suppression test in patients with OCD supports HPA hyperactivity (Cottraux et al., 1984; Catapano et al., 1990), as does increased nocturnal secretion of ACTH and cortisol (Kluge et al., 2007). However, some other studies have shown hyporeactivity of the HPA axis activity in OCD (Zohar et al., 1987; Bailly et al., 1994; Khanna et al., 2001) or no difference (Charney et al., 1988; Hollander et al., 1992; Chappell et al., 1996).

In summary, for some women, hormonally significant events (e.g., onset of menarche, the menstrual cycle, pregnancy, and childbirth) may be associated with an increase in OCD symptoms. However, the hormonal factors underlying such associations are not yet well understood, and it is likely that these factors affect the intensity of OCD symptoms rather than explain its origins. Anecdotal accounts suggest that for some patients with OCD, stressful life events may have preceded the onset of the disorder, which may imply HPA axis hyperactivity in OCD. Despite interesting HPA axis findings related to OCD, these do not seem consistent enough to indicate that dysfunction of this system is the underlying cause of OCD.

4. Immune System Correlates of Obsessive-Compulsive Disorder.

a. Infection and the Immune System in ObsessiveCompulsive Disorder. Swedo et al. (1998) described a subgroup of pediatric patients with OCD who presented a very abrupt onset of symptoms, which appeared to be triggered by a group A Streptococcus infection (Swedo et al., 1998). This syndrome was named pediatric autoimmune neuropsychiatric disorders associated with streptococcal infections (PANDAS) and was proposed as a possible diagnostic subgroup of OCD. However, because a variety of other psychiatric symptoms, including hyperactivity, tics, and separation anxiety (Murphy et al., 2014), also sometimes showed an abrupt onset in apparent association with nonstreptococcal infections, a second syndrome was proposed to encompass such cases. This syndrome was named pediatric acute-onset neuropsychiatric syndrome (PANS). PANS diagnostic criteria include the following: 1) an abrupt onset of OC symptoms or severely restricted food intake; 2 ) the presence of additional psychiatric symptoms that may include anxiety, emotional lability, irritability, behavioral regression, hyperactivity and inattention, sensory or motor abnormalities, or somatic signs such as sleep disturbances; while 3) other neurologic or psychiatric diagnoses conditions, such as Sydenham chorea, lupus cerebritis, or schizophrenia, must be ruled out (Chang et al., 2015). Although infection and altered immune function are suspected to be triggers for PANS, the PANS diagnosis does not require these factors to be present.

Considering that PANDAS/PANS was initially described in children, a valid point of departure would be to ask whether childhood OCD, which presents some phenomenological features that may distinguish it from adult OCD (section III.D.2), is uniquely associated with certain immune alterations. In partial support of such a distinction, a number of investigations have indicated that childhood-onset OCD and/or TS present some immune alterations that have not been reported in adult OCD. Thus, one study of childhood OCD showed increased plasma levels of interleukin (IL)-12 among participants (Gabbay et al., 2009); a separate study of children with OCD or TS showed increased plasma levels of tumor necrosis factor (TNF)- $\alpha$ along with IL-12 in patients, which further increased concurrent with symptom exacerbation (Leckman et al., 2005), whereas 3a third study of children with TS without comorbid OCD showed increased plasma levels of IL-12, TNF- $\alpha$, IL-6, and IL-17 (Yeon et al., 2017). Notably, in one study of adult patients with OCD, the subset of patients who experienced OCD symptom onset as children showed higher plasma IL- 6 and TNF- $\alpha$ levels compared with control subjects, whereas those patients who experienced OCD onset as adults did not differ from controls in this respect (Konuk et al., 2007). Other findings with regard to childhood-onset OCD include increased IL-2 and reduced IL-4 and IL-10 in the cerebrospinal fluid compared with children with schizophrenia or ADHD (Mittleman et al., 1997).

Adult OCD, nonetheless, has been associated with increased plasma levels of soluble TNF receptor (but not TNF- $\alpha$ itself), which were positively correlated with washing compulsions (Fontenelle et al., 2012). In contrast, Denys et al. (2004a) and Fluitman et al. (2010) found decreased ex vivo production of IL- 6 and TNF- $\alpha$ in whole blood samples from adult patients with OCDs. A meta-analysis (Gray and Bloch, 2012) that focused specifically on IL-1B, IL-6, and TNF- $\alpha$ failed to show significant alterations in plasma IL- 6 or TNF- $\alpha$ levels, whereas plasma levels of IL-1B were significantly decreased in the OCD sample compared with controls. Nonetheless, the authors noted that these findings might be confounded by medication and comorbid depression. Controlling for these confounds, Rao et al. (2015) reported increased IL-2, IL-4, IL-6, IL-10, and TNF- $\alpha$ levels in drug- and comorbidity-free patients with OCD compared with age- and sex-matched healthy control subjects.

Importantly, associations between altered immune function and neuropsychiatric symptoms are not limited to childhood. Nor are they unique to OCD, as immune alterations have been associated with other psychiatric conditions, including depression, anxiety, and schizophrenia (Miller et al., 2009; Furtado and Katzman, 2015; Rodrigues-Amorim et al., 2018). 
There have been efforts to investigate in animal preparations the immune effects secondary to streptococcal infection, using rodents immunized with a crude homogenate of group A $\beta$-hemolytic Streptococcus pyogenes (GABHS), or infused with antibodies or sera from human patients. The interpretation of findings from such models needs extra caution, as rodents are not naturally infected with GABHS (Hoffman et al., 2004), and significant differences between protein antigens may exist across species.

Nevertheless, initial studies showed that GABHSimmunized mice produced autoantibodies that crossreacted with several brain regions, including the deep cerebellar nuclei (Hoffman et al., 2004). Animals with greater autoantibody reactivity exhibited increased rearing behavior and IgG deposits in the brain. Passive transfer of antibodies from GABHS-immunized animals to nonimmunized animals (Yaddanapudi et al., 2010) also induced increased rearing behavior, reduced aggression and social interaction, and deficits in motor coordination, consistent with motor coordination deficits and emotional reactivity sometimes reported in PANDAS (Swedo et al., 1998). Subsequently, an independent group of investigators demonstrated that GABHS-immunized mice also displayed a number of perseverative behaviors, impaired prepulse inhibition, reduced concentrations of serotonin in the prefrontal cortex, and inflammatory infiltrates and microglial activation in the anterior diencephalon (Macrì et al., 2015, 2018).

Likewise, a rat model of PANDAS in which animals were immunized with a purified GABHS antigen (M protein type 18; Brimberg et al., 2012) showed impaired motor function as well as increased grooming. Acute administration with haloperidol reduced these symptoms, whereas subchronic (3 days) treatment with paroxetine diminished grooming. In immunized animals, IgG deposition was seen in the striatum, thalamus, and cortex, and cortical dopamine levels were elevated. Sera from exposed rats reacted with human dopamine D1 and D2 receptors, whereas crossreactivity of sera from patients with PANDAS showed a similar pattern of D1 and D2 receptor binding. In another study (Lotan et al., 2014), intrastriatal infusion of IgG that was purified from the sera of rats immunized with GABHS antigen induced similar motor abnormalities but no increases in grooming. Interestingly, these rats did show increased marble-burying behavior, a putative behavioral measure of compulsiveness. Finally, sera from patients with PANDAS or TS infused into either the ventral or ventrolateral striatum of rats did not produce any behavioral abnormalities, raising the issue of antibody cross-species reactivity mentioned earlier (Loiselle et al., 2004).

The start and resolution of some infections, such as cerebral malaria (Idro et al., 2005), Mycoplasma pneumoniae infection (Ercan et al., 2008), and Borna disease virus (BDV) infection (Rotge et al., 2010), may be coupled closely with the appearance and disappearance of OCD symptoms. Moreover, OCD symptoms may be a manifestation of some autoimmune disorders, such as Sydenham chorea (Swedo et al., 1989) and systemic lupus erythematosus (Yu et al., 2008). More generally, the findings that various immune markers are altered in patients with OCD (da Rocha et al., 2008; Pearlman et al., 2014) suggest two possible mechanisms by which infection and immune activation could yield OCD pathophysiology.

One possible mechanism may be similar as in tuberculosis-namely, that a specific pathogen causes OCD, just like tubercle bacillus is the causal agent of tuberculosis. Symptoms and pathophysiology of tuberculosis are the result of the bacterium having a preference for certain body organs (e.g., lungs) and immune processes therein acting to contain the bacterium. The ensuing battle yields "collateral damage" in the afflicted organs and physiology, which is manifested as diseasespecific symptoms. Rotge et al. (2010) theorized that a similar "process by which infection becomes disease" (Barnes, 2000, p. 439) may yield OCD. Specifically, considering reports (Bode et al., 1995) that BDV infection may play a role in psychiatric disorders, Rotge et al. (2010) noted that the virus has a preferential neurotropism for brain regions of relevance to OCD and that the ensuing regional immune responses may result in a region-specific malfunction as a by-product of those immune processes. Of the many plausible mechanisms by which immune reactions could perturb neuronal function (for a recent review, see Kapadia and Sakic, 2011), Rotge et al. (2010) highlighted the possibility of glutamate neuro-excitotoxicity, considering the genetic findings of an association of the glutamate transporter gene SLC1A1 with OCD (Arnold et al., 2006; Wu et al., 2012) and recognition that glutamate is both a neurotransmitter and an immunomodulator. Hence, according to Rotge et al. (2010), genetic alterations of the glutamatergic system could facilitate BDV-induced regional immunopathological reactions in some individuals (Rotge et al., 2010), resulting in hyperactivity of basal ganglia loops (Wu et al., 2012) and thereby producing OCD symptoms.

Another possible mechanism by which immune activation could yield OCD pathophysiology is related to autoimmune processes themselves, rather than stemming from local reactions to neurotropic pathogens. A prime example is PANDAS, where antistreptococcal antibodies are thought to crossreact with neuronal basal ganglia antigens (Swedo et al., 1997; Eisen et al., 2001) and perturb neuronal function (Kapadia and Sakic, 2011). Accordingly, OCD ensues when humoral immunity targets neuronal elements of the basal ganglia neurocircuit, and the resultant malfunction consists of overly persistent and uncontrolled circuit activity characteristic of OCD pathophysiology (Szechtman et al., 2014). Several studies have associated such antineuronal 
antibodies in plasma with PANDAS, including antibodies that react with dopamine receptors, lysogangliosides, and tubulin, and which result in the activation of the calmodulin-dependent protein kinase II signaling pathway in human neuronal cells (reviewed in Cunningham et al., 2015).

It is also possible that both of the foregoing immune mechanisms, as well as possibly others, are involved. In addition to autoimmunity, other immune-mediated processes may participate in generating the PANDAS/ PANS symptoms. Thus, microglia activation and inflammation within the caudate and putamen have been associated with childhood TS and PANDAS (Kumar et al., 2015); activated microglia might result in neuronal loss and alterations in synaptic pruning (Prinz and Priller, 2014; Frick and Pittenger, 2016). Investigations that have focused on the possible relationship between cytokine-induced sickness behavior and depression have revealed a number of cytokine actions on brain function that may be relevant to OCD and other neuropsychiatric symptoms, including increased tryptophan-kynurenine metabolism and the reduction of serotonin and dopamine synthesis, increasing serotonin reuptake, increasing extrasynaptic glutamate release and excitotoxicity, and reducing neurogenesis (reviewed in Miller et al., 2009).

Three lines of evidence suggest that future research might profitably focus on the role of microglia and macrophages in the pathogenesis and pathophysiology of OCD. The first line of evidence comes from animal model studies in which Hoxb8 mutant mice developed excessive and pathologic grooming proposed to model OCD (Greer and Capecchi, 2002). The truly remarkable follow-up studies provided the surprising finding that the compulsive grooming was not driven by a hyperfunction in a neural circuit mediating grooming but rather by mutant microglia, since the only detectable cells derived from the Hoxb8 cell lineage in the brain were microglia (Chen et al., 2010).

The second line of evidence comes from studies showing that experimental manipulations of psychologic factors are associated with changes in microglia in selected brain areas. For instance, acute social defeat, but not chronic social defeat, increased resident microglial proliferation in mice, which occurred selectively in telencephalic stress-related brain areas (Lehmann et al., 2016). Finally, of particular relevance to the QS animal model of OCD (Szechtman et al., 1998), there is evidence of functional expression of dopamine receptors in mouse and rat microglia, in culture and brain slices (Färber et al., 2005). In addition, studies show that dopamine regulates the synthesis of inducible NOS, IL-1 $\beta$, and IL-6 in microglial cells (Färber et al., 2005; Trudler et al., 2014), and that dopamine is a potent modulator of M2-to-M1 macrophage polarization by signaling through dopamine D2 receptors (Qin et al., 2015).
It is possible that infection-related psychiatric symptoms might represent an exaggerated version of a more general adaptive mechanism by which internally generated cues associated with infection promote the expression of certain protective cognitions and behaviors that are associated with OC symptom expression. Consistent with this proposal, it has been reported that latent toxoplasma infection is not only more prevalent in subjects with an OCD diagnosis, but it is also associated with increased OC symptoms in nonclinical subjects that did not meet the diagnostic criteria of OCD (Miman et al., 2010; Flegr and Horáček, 2017). Fontenelle et al. (2012) proposed that the observed positive correlation between plasma levels of soluble TNF receptor and severity of washing symptoms might reflect the immune-mediated activation of a "behavioral macro" that protects against infectious agents. Similarly, cytokines have been suggested to promote putatively adaptive behavioral and cognitive patterns associated with sickness behavior: a decrease in motivation (via cytokine effects on basal ganglia circuits) and an increase in vigilance, conflict detection. and arousal (mediated by cytokine action on the ACC) (reviewed in Miller et al., 2009).

Finally, it is important to distinguish between two possibilities. One possibility is the actual infection of the organism, leading to pathogen-caused tissue damage and immunopathology that may possibly yield OCD behavior, as just discussed. However, another important possibility is the organism's detection, prior to being infected, of the potential risk of exposure to pathogens, such as might be cued by foul smells, which may also lead to preventative and precautionary behaviors characteristic of OCD. Such disease-avoidance behavioral adjustments, which are both innate and learned, are an essential preimmune system component of the organism's defense strategy against disease (Medzhitov et al., 2012). The theme of managing potential danger for OCD is discussed in more detail in section IV.

In summary, there is intriguing but complex evidence linking infection and immune system changes to OCD. Best known is the PANDAS/PANS syndrome of sudden onset of pediatric OCD symptoms, but there is some evidence linking infection and immune system changes to adult OCD as well. Several promising underlying neurobiological mechanisms have been proposed, which will serve as important leads for further research. However, it is important to distinguish between sequelae of actual infection versus adaptations to protect against the risk of future infection.

b. Neuroinflammation. It has been suggested that multiple "hits" may be required to initiate a clinical syndrome in susceptible individuals who later go on to develop a full-blown illness such as schizophrenia, depression, and other anxiety and stress-related 
disorders (Davis et al., 2014; Möller et al., 2015). Davis et al. (2014) note that such hits potentially first occur in utero, leading to neuronal disruption, epigenetic changes, and the establishment of an abnormal inflammatory response. The development of OCD may therefore be viewed as a neuroprogressive response to early-life adversity driven by changes in proinflammatory cytokines, TRYCAT metabolism, the microbiome, reactive oxygen and nitrogen species, and overt activation of the glutamate-NMDA receptor system, for example (Davis et al., 2014).

In this regard, patients with moderate to severe OCD may show favorable response to various neuroprotective, anti-inflammatory, and antiglutamatergic compounds as adjunctive treatments, such as celecoxib (Shalbafan et al., 2015), minocycline (Esalatmanesh et al., 2016), memantine (Ghaleiha et al., 2013), and riluzole (Emamzadehfard et al., 2016), often enabling more rapid improvement in OCD symptoms (see also section III.A.3).

\section{E. Conclusions}

In this section, we have reviewed a wide range of factors that have been implicated in the expression of OCD and OCD-like behaviors. Table 3 summarizes the factors and the major hypothesized mechanisms by which they may affect OCD. These factors, drawn from an extensive literature, include neurotransmitters (e.g., serotonin, dopamine, glutamate, and GABA modulators), endogenous processes (e.g., oxidative stress, the microbiome, and neurosteroids), patterns of brain functioning (as revealed by brain imaging), and etiological variables (e.g., genetics, age of onset, and the hormonal and immune systems).

Our impression is that the motivation for much of this work is the hope that somewhere among all of these findings will lie the clues that will lead to an agent with a strong and specific, corrective effect on OCD—a "magic bullet," so to speak (Woody et al., 2019). From this perspective, the diverse and sometimes contradictory results of such an exhaustive search may seem disappointing, in that no such crucial leads have been forthcoming. However, the underlying hopes of discovering a magic bullet may be misguided and in need of reexamination, as discussed later in section $\mathrm{V}$.

The myriad, different findings relating to OCD, spanning multiple levels and systems, also cry out for some unifying theoretical perspective that could be used to integrate them and give them a coherence they otherwise sorely lack. In this respect, the current situation for OCD is, unfortunately, similar to that for many other psychiatric disorders. Some critics have recently argued that the atheoretical approach to psychiatric research over the last few decades, as typified by the DSM, has proven to be a failure and thus it is time to adopt alternative approaches. We now turn to these ideas in section IV.
TABLE 3

Summary of neurobiological factors implicated in OCD, hypothesized mechanism by which they exert an effect on OCD, and the section number where the relevant research is discussed

\begin{tabular}{|c|c|c|}
\hline Implicated Factor & Hypothesized Mechanism & Section \\
\hline \multirow{4}{*}{$\begin{array}{l}\text { Serotonin } \\
\text { neurotransmission }\end{array}$} & Neurotransmitter deficit & III.A.1 \\
\hline & Low 5-HT transporter & \\
\hline & $\begin{array}{l}\text { Altered function of } 5 \text {-HT1A } \\
\text { receptors }\end{array}$ & \\
\hline & $\begin{array}{l}\text { Hypofunction of } 5 \text {-HT2C } \\
\text { receptors }\end{array}$ & \\
\hline \multirow[t]{2}{*}{$\begin{array}{l}\text { Dopamine } \\
\text { neurotransmission }\end{array}$} & $\begin{array}{l}\text { D2 and/or D1 receptor } \\
\text { activity }\end{array}$ & III.A. 2 \\
\hline & Interaction with serotonin & \\
\hline \multirow[t]{3}{*}{$\begin{array}{l}\text { Glutamate and GABA } \\
\text { neurotransmission }\end{array}$} & $\begin{array}{l}\text { Altered glutamate } \\
\text { concentration }\end{array}$ & III.A.3 \\
\hline & $\begin{array}{l}\text { Faulty glutamate } \\
\text { transporters }\end{array}$ & \\
\hline & GABA-glutamate imbalance & \\
\hline \multirow[t]{2}{*}{ Oxidative stress } & Increased lipid peroxidation & III.B.1 \\
\hline & Disturbances in cortical GSH & \\
\hline $\begin{array}{l}\text { Phosphoinositide } \\
\text { hyperactivity }\end{array}$ & Insufficient MI & III.B.2 \\
\hline NO-cGMP pathway & Increased levels of $\mathrm{NO}$ & III.B. 2 \\
\hline cAMP-dependent pathway & $\begin{array}{l}\text { Supersensitive AC activity } \\
\text { Increased PDE } 4 \text { activity }\end{array}$ & III.B.2 \\
\hline \multirow[t]{2}{*}{ Brain-gut-microbiota axis } & $\begin{array}{l}\text { Specific alterations in the gut } \\
\text { microbiome }\end{array}$ & III.B.3 \\
\hline & $\begin{array}{l}\text { Altered metabolism in the } \\
\text { TRYCAT pathway }\end{array}$ & \\
\hline Neurosteroid dysregulation & $\begin{array}{l}\text { Modified gene expression and } \\
\text { neuronal excitability }\end{array}$ & III.B.4 \\
\hline Oxytocin & $\begin{array}{l}\text { Activates threat detection } \\
\text { circuits }\end{array}$ & III.B. 4 \\
\hline Neurotrophic factors & $\begin{array}{l}\text { BDNF reduced, possibly due } \\
\text { to genetics }\end{array}$ & III.B.5 \\
\hline $\begin{array}{l}\text { Regional neuroanatomical } \\
\text { changes }\end{array}$ & $\begin{array}{l}\text { Alteration of a circuit } \\
\text { composed of cortex-basal } \\
\text { ganglia-thalamus-cortex } \\
\text { pathways }\end{array}$ & III.C. \\
\hline $\begin{array}{l}\text { Specific genes or } \\
\text { polymorphisms }\end{array}$ & $\begin{array}{l}\text { Result in alterations of } \\
\text { serotonergic, dopaminergic, } \\
\text { and glutamatergic } \\
\text { signaling }\end{array}$ & III.D.1 \\
\hline Age of onset & $\begin{array}{l}\text { Childhood onset may be } \\
\text { distinct }\end{array}$ & III.D. 2 \\
\hline Sex hormones & $\begin{array}{l}\text { Alter serotonin } \\
\text { neurotransmission }\end{array}$ & III.D.3 \\
\hline Stressful life events & $\begin{array}{l}\text { Induction of HPA } \\
\text { hyperactivity }\end{array}$ & III.D.3 \\
\hline Streptococcal infection & Altered immune function & III.D.4.a \\
\hline \multirow{5}{*}{$\begin{array}{l}\text { Other infections, } \\
\text { autoimmune and } \\
\text { inflammatory processes }\end{array}$} & $\begin{array}{l}\text { Glutamate neuro- } \\
\text { excitotoxicity }\end{array}$ & III.D.4.a \\
\hline & $\begin{array}{l}\text { Antibodies crossreact with } \\
\text { brain antigens }\end{array}$ & \\
\hline & Microglia activation & \\
\hline & $\begin{array}{l}\text { Change in TRYCAT } \\
\text { metabolism }\end{array}$ & \\
\hline & $\begin{array}{l}\text { Disease-avoidance behavioral } \\
\text { adjustments }\end{array}$ & \\
\hline Neuroinflammation & $\begin{array}{l}\text { Cascade of cumulative } \\
\text { responses }\end{array}$ & III.D.4.b \\
\hline
\end{tabular}

\section{Toward a New Perspective on Preclinical Research}

\section{A. Alternative Frameworks for Research on Obsessive- Compulsive Disorder}

1. The Diagnostic and Statistical Manual of Mental Disorders versus the Research Domain Criteria. Since the advent of the DSM-III in 1980 and continuing through 
its successive revisions (e.g., the DSM-5: American Psychiatric Association, 2013), the DSM diagnostic criteria for OCD have served as the linchpin on which research on OCD has been organized. These diagnostic criteria, like those for other mental disorders, rely on descriptive phenomenology, based mainly on the person's self-report as conveyed to a clinician. A central emphasis in the development of these criteria was the need for good inter-rater reliability as a necessary precondition for adequate measurement; in contrast, there has been less focus on issues of validity. In addition, the criteria are atheoretical, avoiding any theory of the underlying causes and nature of OCD.

The DSM as the gold standard for defining what OCD is has posed some major difficulties for preclinical researchers seeking to develop and validate models of OCD. In particular, the DSM strongly emphasizes phenomenology, especially obsessions, that cannot generally be observed in laboratory animals. Instead, researchers have needed to do a careful analysis of behavioral patterns to support the argument that an animal model approximates human OCD in some important ways (e.g., Szechtman et al., 1998; Ben-Pazi et al., 2001; Joel, 2006; Wolmarans et al., 2018). However, because the DSM view of OCD is atheoretical, it is difficult to know what to use as a conceptual bridge to adequately link patterns of animal behavior to human OCD.

The longstanding use of DSM diagnostic criteria as the foundation for research on mental disorders has recently come under vigorous criticism. The guiding hope underlying reliance on the DSM was that reliable categorization of mental disorders based on phenomenology would serve as the starting point for developing theories and discovering underlying causes; however, many critics have pointed out that this hope has remained unfulfilled (Hyman, 2010; Phillips et al., 2012; Casey et al., 2013; Cuthbert, 2014). Particularly disturbing is the failure of DSM-defined psychopathology to connect with advances in neuroscience and genetics. For example, Michael First noted, "After 30 years of intensive efforts by the research community, not a single biological marker or gene has been discovered that is useful in making a psychiatric diagnosis. ... It is likely that the categorical descriptive DSM system itself is at least partly to blame" (Phillips et al., 2012, p. 8). Similarly, another discussant, Joseph Pierre, remarked, "Post-DSM-III etiologic research has yielded mostly inconsistent or heterogeneous findings ... DSM diagnoses are constellations of symptoms for which there are probably myriad etiologies" (Phillips et al., 2012, p. 4). In addition to the problem of large heterogeneity among people with the same diagnosis, there are considerable issues of comorbidity (e.g., Szechtman and Woody, 2006), leading critics to conclude that DSM diagnoses "have increasingly been shown not to represent valid disease entities" (Cuthbert, 2014, p. 28), are "poor mirrors of nature" (Cuthbert, 2014, p. 810), and fail to define "natural kinds, real entities that exist independently of any particular rater" (Hyman, 2010, p. 156). Indeed, critics have argued that the DSM is now seriously hindering research in psychopathology (Phillips et al., 2012; Casey et al., 2013; Hengartner and Lehmann, 2017); for instance, Hyman (2010) has characterized DSM diagnostic criteria as an "unintended epistemic prison ... palpably impeding scientific progress" (p. 157).

In view of these shortcomings, what might be a promising alternative to the DSM as the organizing framework for psychopathology research? A bold step toward such an alternative is the recently proposed $\mathrm{RDoC}$ project (Cuthbert, 2014). The $\mathrm{RDoC}$ comprises two main themes. First, research should focus more on symptoms themselves as problematic patterns of behavior, instead of broader DSM-like diagnoses, and should take a dimensional approach that encompasses a wider range of manifestations of such behaviors, or "the full range of variation, from normal to abnormal" (Casey et al., 2013, p. 812). Second, psychopathology research should be more solidly based on neuroscience and genetics. To facilitate this shift, the devisors of the RDoC specified a set of basic types of brain systems that should guide and organize psychopathology research; these are Negative Valence Systems, Positive Valence Systems, Cognitive Systems, Systems for Social Processes, and Arousal/Modulatory Systems. Because each of these basic brain systems can be investigated at any of several distinct levels, the devisors also advanced an $\mathrm{RDoC}$ matrix, in which the types of brain systems are the rows and the following levels of investigation (Units of Analysis) are the columns: Genes, Molecules, Cells, Circuits, Physiology, Behavior, and Self-Reports. Clearly at the center of these levels of investigation are brain circuits, with genes, molecules, and cells as circuit constituents, and with physiologic responses, behavior, and self-reports as the circuit outputs. The following is a pithy summary of the proposed $\mathrm{RDoC}$ approach:

RDoC asks [these] questions: "What is the normal distribution for a certain trait or characteristic; what is the brain system that primarily implements this function; and, how can we understand, at various levels of mechanism, what accounts for the development of dysregulation or dysfunction in these systems along normal-to-abnormal dimensions? (Cuthbert, 2014, p. 31)

2. An Adaptationist Reorientation of the Research Domain Criteria Approach for Obsessive-Compulsive Disorder Research. In attempting to match the proposed RDoC approach specifically to research on OCD, the fit is intriguing but seems incomplete. First, the shift toward a dimensional approach makes sense, because OCD symptoms are widespread in the general population (if in a less extreme form) and reasonably normally distributed in degree (for a review, see 
Szechtman and Woody, 2004). However, the proposed $\mathrm{RDoC}$ approach does not really make clear how to conceptualize and make use of this apparent continuity from normal to abnormal. Second, the shift to a focus on underlying brain systems seems promising and potentially generative. However, OCD symptoms would appear to involve most (or perhaps all) of the basic brain systems specified in the RDoC matrix: for example, Negative Valence Systems (e.g., anxiety), Cognitive Systems (e.g., perception of potential threat, shifts in attention, etc.), Systems for Social Processes (e.g., perception of the self and of others), and Arousal/ Modulatory Systems (e.g., sustained arousal). In other words, these RDoC-specified systems do not offer much specificity about where to search for understanding OCD symptoms.

Instead, we propose a reorientation of the $\mathrm{RDoC}$ approach, at least with regard to investigating OCD. The guiding theme is encapsulated in the well known assertion, "Nothing in biology makes sense except in the light of evolution" (Dobzhansky, 1973). According to this adaptationist viewpoint, "the architecture of each evolved system should reflect the computational requirements of the adaptive problem that selected for its design" (Sznycer et al., 2017, p. 56). More specifically, evolution selected for brain networks that recruit and coordinate brain systems in ways that deal with particular classes of adaptive problems that have been crucial for survival (Tooby and Cosmides, 1990; Tooby et al., 2008). Such a functional network is not located within any particular RDoC-specified brain system; instead, it "entrains many different 'components,' adjusting their thresholds and parameters, and shuts off others" (Sznycer et al., 2017, p. 57). Accordingly, we propose that the generative "natural kind" in OCD is closely tied to a particular underlying class of problems in adaptation, and it consists of the functional network of component brain systems recruited through evolution to manage this class of problems.

Most OCD symptoms have a very clear core theme: concern about potential dangers that might befall the self and others, and engagement in precautionary and preventative behaviors that would ward off such harm. Indeed, actions characteristic of OCD, such as checking and washing, are widespread not only across human cultures but also across different animal species, indicating that they have substantial evolutionary importance. These considerations suggest that the symptoms of OCD can be conceptualized as the dysfunctional release of ancient fixed-action patterns, perhaps hardwired in the basal ganglia (Rapoport, 1989; Wise and Rapoport, 1989). As Rapoport and Fiske (1998) remarked, "Such specificity in the 'hard wiring' of human behaviors as basic to everyday functions as cleaning, checking, and ordering, or concern about dangers to self or others, suggests a biological basis for universal categories of certain thoughts and rituals" (p. 160).
This class of adaptive behaviors, involving the management of risk from relatively low probability but potentially lethal events, such as contagion by disease or attack by predators, is essential for survival, and hence it is highly plausible that evolution strongly shaped brain networks for them. Accordingly, ethologically oriented psychologists have outlined a motivationally fundamental brain system for the detection and amelioration of potential danger (e.g., Adams, 1979; Bolles and Fanselow, 1980; Masterson and Crawford, 1982; Gilbert, 1989; Marks and Nesse, 1994). This biologically ancient system has been given somewhat different names by different investigators. Abed and de Pauw (1998) called it the involuntary risk scenario generating system, Trower et al. (1990) called it the defense system, Boyer and Liénard (2006) called it the hazard-precaution system, and Szechtman and Woody (2004) called it the security motivation system (SMS), which is the name we will use in this review.

Ethological psychologists have done extensive research, reviewed in Szechtman and Woody (2004) and in Woody and Szechtman (2011), into how animals manage potential threats, such as the threat of predation. This research provides essential clues about the nature and properties of the underlying brain network that would be required, and so is very briefly summarized here:

- Activating stimuli and appraisal process: The assessment of potential danger requires specialized processors for the detection and evaluation of subtle, uncertain, and indirect cues suggesting hidden risks. These cues and the required processing are quite different from those for detecting imminent or actual danger (such as the presence of a predator or pathogens inside the host).

- Nature of activation and motivational state: Detection of even weak cues suggesting potential danger readily activates a motivational state of vigilance and apprehension, and this state dissipates only relatively slowly even in the absence of further, validating cues. This internal state is phenomenologically distinct from the experience elicited by imminent danger.

- Security-related behavior: The resulting behavioral outputs consist of precautionary behaviors, such as checking, which involve probing and manipulating the environment to obtain further information about possible risks, and preventative behaviors, such as washing, which may help to ameliorate any effects of the danger if it eventuates. These behaviors are very distinct from responses to imminent danger, which include escape and fight-or-flight responses (Cannon, 1927) and "sickness behaviors" (Hart, 1988, 1990; Medzhitov et al., 2012; Felger and Treadway, 2017; Vichaya and Dantzer, 2018).

- Goal attainment and deactivation: Unlike some other types of motivated behavior, security-related 
behavior is "open-ended" in the sense that the environment does not usually yield any consummatory stimulus to signal goal attainment. That is, even if the behavior is worthwhile, the consequence is a nonevent-nothing happens. Instead, it is engagement in the security-related behavior itself that appears to provide the internal feedback signal to terminate the motivational state and hence security-related behavior.

\section{B. The Security Motivation System}

1. Obsessive-Compulsive Disorder as the Dysfunction of a Normal Motivational System. Guided by these insights and taking a systems-design approach, Szechtman and Woody (2004) advanced a model of the SMS, laying out its main functional components and their interconnections, as shown in Fig. 1. The first functional component, Appraisal of Potential Danger, performs a perceptual-cognitive analysis of environmental stimuli to evaluate potential danger to the self or others. If potential danger is detected, it sends an excitatory signal to the second component, Security Motivation, which activates a motivational state with a protracted half-life and produces two coordinated outputs. One output, labeled Anxiety/Wariness, provides a positive feedback loop to the Appraisal of Potential Danger component, tending to sustain the appraisal by biasing the cognitive-perceptual processes. The other is an excitatory signal to a third major component, Security-Related Programs, which coordinates speciestypical motor and output-oriented programs for the protection of the self or others, such as checking or washing. Subsequent engagement in the activated behaviors generates the next functional component, Motor and Visceral Output. The model proposes that this output provides two routes of negative feedback: an internal terminator signal, which Szechtman and Woody named Yedasentience, that normally inhibits activity of both the Security Motivation and Appraisal subsystems, and the enhancement of Safety Cues, which provides another, slower route for inhibition of the Appraisal subsystem.

The understanding of the normal functioning of the SMS and its component processes provides the crucial foundation for explaining how dysfunction would lead to the expression of psychopathological symptoms (Kendler, 2008). Specifically, Szechtman and Woody (2004) proposed that OCD results from faulty regulation in this biologically primal system. They argued that OCD is a dysfunction in the deactivation of the system, such that potential-threat concerns are elicited in the normal way, but once these concerns are activated, security-related thoughts and actions persist abnormally long because engagement in security-related behaviors is abnormally weak in shutting them down. In accordance with this theory, the Xs in Fig. 1 indicate where weakening of functional connections would yield OCD. A blockage from Motor and Visceral Output to Yedasentience would interfere with normal deactivation of the activity of the Security Motivation and Appraisal of Potential Danger components, such that performance of precautionary behaviors would fail to provide the normal dampening of anxiety and the cognitive-perceptual appraisal of potential danger and would hence persist for abnormal lengths of time. A similar effect would result if the Security Motivation component is insensitive to Yedasentience, as represented by the $\mathrm{X}$ at the top of the Security Motivation module. Alternatively, a blockage from

\section{Security Motivation System}

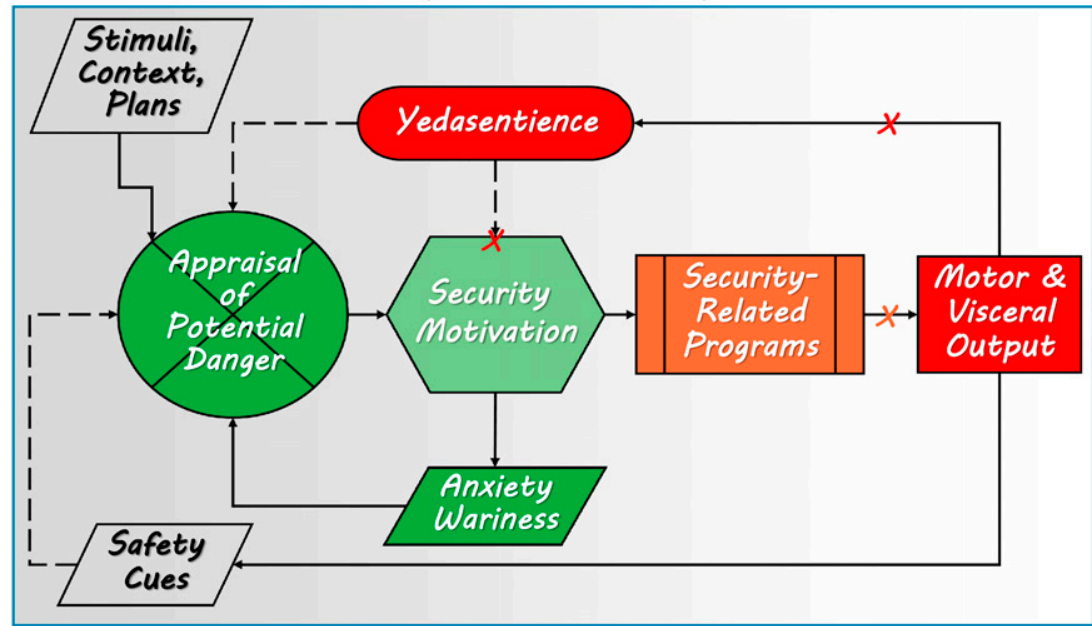

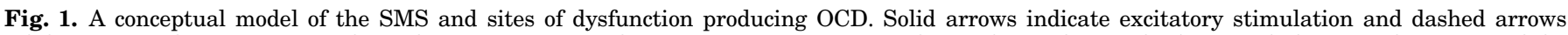

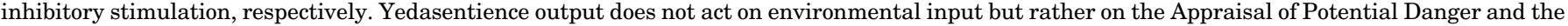

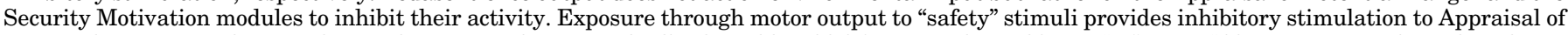

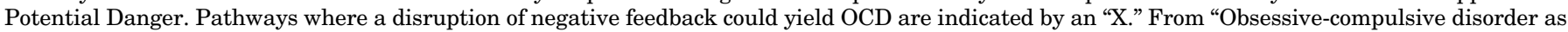

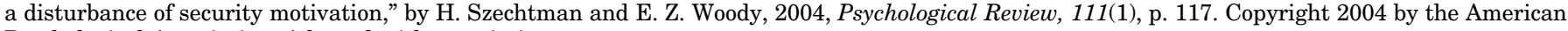
Psychological Association. Adapted with permission. 
Security-Related Programs to Motor and Visceral Output would interfere with engagement in the precautionary behaviors that serve as the basis for inhibitory feedback in the SMS, which may correspond to the "pure obsessional" type of OCD, in which compulsive behavior is lacking (Emmelkamp and Kwee, 1977; Stern, 1978; Salkovskis and Westbrook, 1989; Steketee, 1993).

Parenthetically, Taylor et al. (2005) critiqued this theory of OCD by arguing that OCD, in essence, is not a dysfunction of termination (i.e., a stopping problem) but is instead a dysfunction of excitation (i.e., a starting problem) (Woody and Szechtman, 2005). This distinction was generative, in that subsequent research showed decisively that these critics were wrong: patients with OCD differ strongly from normal controls in their stopping dynamics, but not in their starting dynamics (Hinds et al., 2012, 2015).

2. The Systems-Design Approach to ObsessiveCompulsive Disorder and the Main Themes of the Research Domain Criteria. This systems-design approach to understanding OCD, as represented in Fig. 1, clearly addresses both main themes of the $\mathrm{RDoC}$ project (Cuthbert, 2014), but in a somewhat different and, we believe, more generative way. Recall that one of these themes was that psychopathology research should focus on symptoms as patterns of behavior of interest in themselves and take a dimensional view, explicitly linking the normal with the abnormal; however, the proposed RDoC approach did not make clear how to conceptualize such continuity from normal to abnormal. In our theoretical framework, the relation between normal and pathologic is that the same specific biologically evolved functional network underlies both, but psychopathology results from faulty dynamics in this underlying network (Kendler, 2008). In psychopathology, regulatory dysfunction leads to the exaggeration of normal patterns, because when these behaviors are less closely and appropriately regulated, they appear in simpler, but magnified form (Teitelbaum and Pellis, 1992; Teitelbaum, 2012). In summary, OCD does not obey different rules, but faulty dynamics come into play, allowing us to predict how the normal system would behave given different kinds of perturbations. This generative linkage between the normal system and pathology means that the study of normal behavior illuminates psychopathology; moreover, the study of psychopathology, in turn, illuminates the normal, because some of its properties appear in a simpler or starker form (Jaspers, 1963).

It is important to emphasize that the link between normal and abnormal has to do with faulty dynamics; as such, this linkage represents much more than a mere extremity on underlying dimensions. Consider, for example, personality disorders, for which it has long been recognized that a crucial contributor to disorder is unusually high or low levels on personality dimensions that are normally distributed in the general population.
Yet it is faulty learning that is posited in theories of the etiology of personality disorders; this faulty learning is partly provoked by the extreme personality traits leading to dysfunctional patterns of interpersonal regulation (e.g., maladaptive transaction cycles; Kiesler, 1996), which contribute to the development of the psychopathology and sustain it.

A useful illustration of dysfunctional dynamics would be a heating system regulated by a thermostat. Even such a simple system has distinct dynamics for turning on versus turning off (called hysteresis). If the thermostat is sticky at the high end, delaying stopping the furnace, there will be periodic episodes of excessive heat; whereas if the thermostat is sticky at the low end, delaying starting the furnace, there will be periodic bouts of excessive cold. If a window is broken out near the thermostat, some rooms will be kept excessively hot. However, in all three of these scenarios, outwardly the system continues to function lawfully; it is its dynamics that are perturbed.

There is another important way in which study of the normal functioning of the SMS informs understanding of the abnormal-namely, with regard to the range of symptom forms and contents that constitute dysfunctional expressions of the system and hence share common origins functionally. The history of DSM-like conceptions of OCD has been characterized by somewhat unending debates over the boundaries of OCD, such as how (or even whether) OCD is related to anxiety disorders and various impulse disorders (e.g., trichotillomania). From an adaptationist perspective, the underlying question is about what range of adaptive problems are subserved by a common, evolved system architecture, and this is an empirical question to be resolved by careful study of the normal system, not a matter of definitional fiat at the level of psychopathology.

Recall that a second main theme of the $\mathrm{RDoC}$ project was that psychopathology research should have a much stronger grounding in neuroscience; however, we noted that OCD symptoms did not fit insightfully into any of the RDoC-specified research targets for basic brain systems. In contrast, in our theoretical framework, specification of the functional components of the SMS network (as in Fig. 1) yields generative hypotheses about the brain circuits (see Fig. 2) and neurophysiology (see Fig. 3) that subserve these functions, and these hypotheses cut across multiple RDoC-specified brain systems. The set of interlocking hypotheses represented by Figs. 2 and 3 make use of what is known from basic neuroscience about the brain systems that could perform these component functions, as explained in detail in Szechtman and Woody (2004) and Woody and Szechtman (2011). Indeed, it might be more accurate to call the SMS a network rather than a system, to distinguish it from what the RDoC labels as a "brain system." As a functional network, the SMS is hypothesized to recruit and organize several component brain systems, most of 


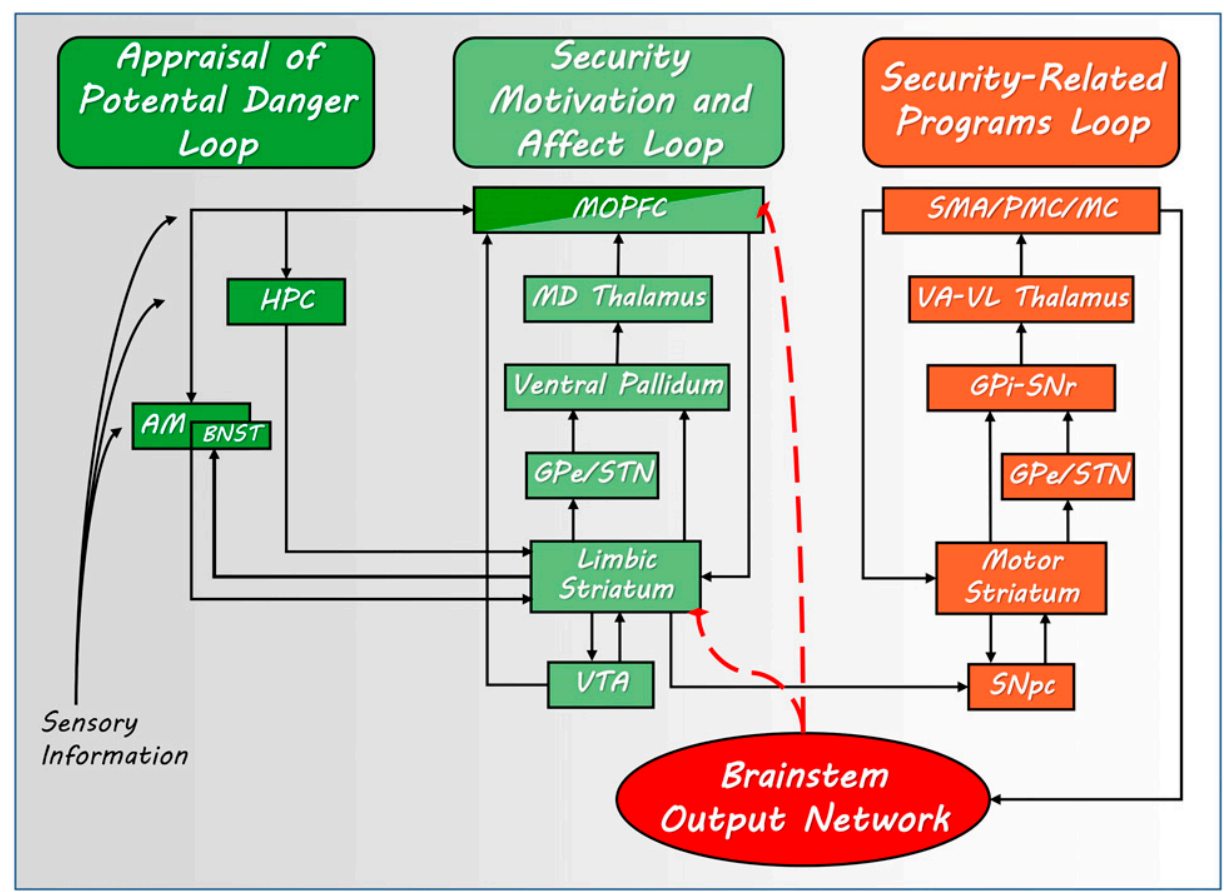

Fig. 2. A neural circuit model of the SMS. Each of the four distinct subcircuits (loops) subserves one of the functional components in Fig. 1 and is identified by corresponding colors. The dashed line indicates possible sites of yedasentience feedback inhibition. AM, amygdala; BNST, bed nucleus of the stria terminalis; GPe, external segment of the globus pallidus; GPi, internal segment of the globus pallidus; HPC, hippocampus; MC, motor cortex; MD Thalamus, mediodorsal thalamic nucleus; MOPFC, medial prefrontal cortex and orbital prefrontal cortex; PMC, premotor cortex; SMA, supplementary motor area; SNpc, substantia nigra pars compacta; $\mathrm{SNr}$, substantia nigra pars reticulata; VA, ventroanterior thalamic nucleus; VL, ventrolateral thalamic nucleus; VTA, ventral tegmental area. From "Obsessive-compulsive disorder as a disturbance of security motivation," by H. Szechtman and E.Z. Woody, 2004, Psychological Review, 111(1), p. 120. Copyright 2004 by the American Psychological Association. Adapted with permission.

which are not, so to speak, "for SMS use only" (Anderson and Finlay, 2014; Anderson, 2016).

This approach is consistent with the recent shift of focus in neuroscience from a relatively narrow localizationist perspective to one that considers the entire functional network (e.g., Boes et al., 2015; Darby et al., 2017; Shine and Poldrack, 2018). As Kendler (2008) argued, brain networks are unlikely to be simple additive mechanisms; instead, they are likely to include "multiple nonlinear interactions and causal loops" (p. 695). This is an important property of complex adaptive systems more generally. As West (2017) pointed out, "A typical complex system is composed of myriad individual constituents or agents that once aggregated take on collective characteristics that are usually not manifested in, nor could easily be predicted from, the properties of the individual components themselves" (p. 21).

Finally, the perspective we have advanced here has important implications for the role of animal models in preclinical research on OCD. We posit that research using animal models should shift away from the attempt to imitate the descriptive phenomenology of psychiatric disorders and toward the experimental investigation of theories of psychiatric disorders, including their neurobiology and therapeutics (Kendler, 2008; Hyman, 2010; Nestler and Hyman, 2010). As Davis and Isles (2014) have argued, "What is needed is a shift in emphasis, from seeking to ally an animal model to a specific disorder, to one focused on a more systematic assessment of the neurobiological and behavioural outcomes of any given genetic or environmental manipulation" (p. 1933). The SMS as a functional network is influenced by diverse factors, such as genetics, environmental circumstances, learning, and drugs, which exert effects at diverse levels, such as neuroanatomy, component programs and circuits, and neurochemistry. Animal research plays a vital role in elucidating the underlying mechanisms in this functional network and its normal dynamics, as well as ascertaining the effects of environmental conditions and learning on it, and evaluating its plasticity, such as possible ways to boost aspects of the system and thereby improve its dynamics when they are dysfunctional. Of course, there may be uniquely human elaborations of how such a network operates-as just one example, Wiebe (2013) has proposed that religions emerged to serve a hazard-precautionary role. However, the presence of a biologically fundamental functional network as a commonality between humans and other animals offers pivotal opportunities for understanding its underlying nature and properties.

\section{Key Aspects of the Security Motivation System Theory of Obsessive-Compulsive Disorder}

Although the SMS theory is one among several theories of OCD, it organizes a great deal of material in 


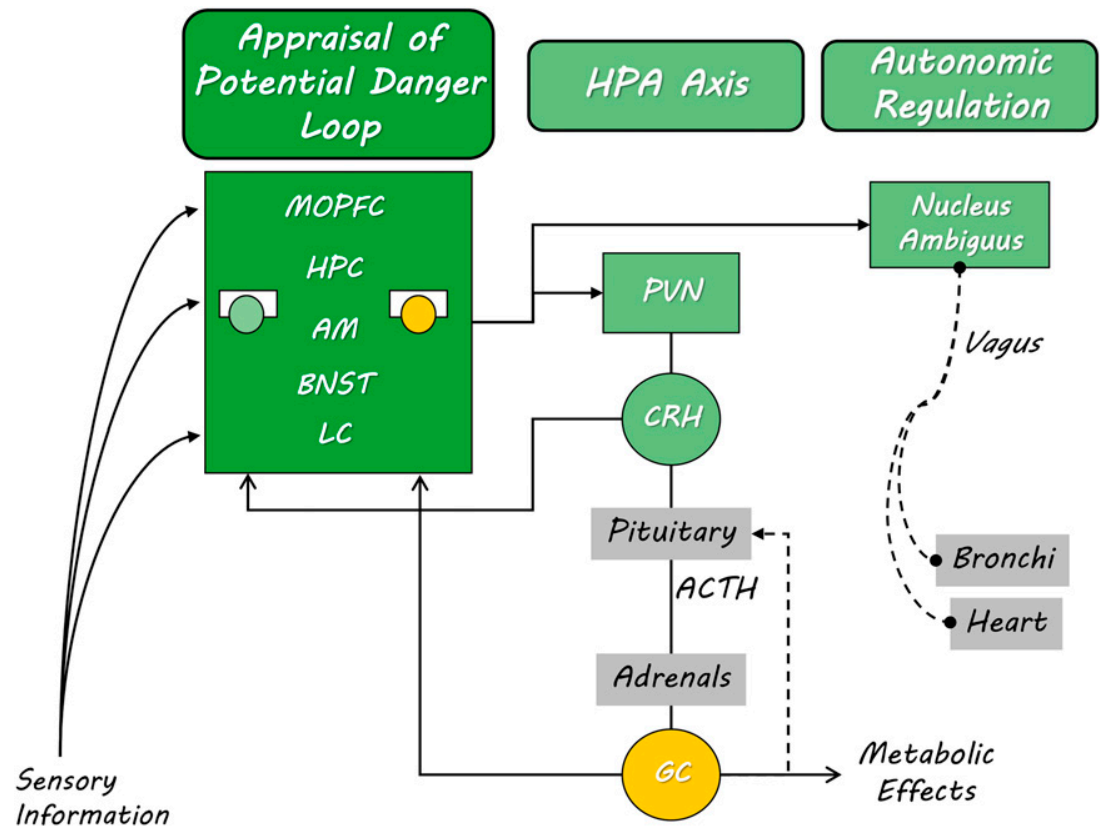

Fig. 3. The physiologic network associated with security motivation. The Appraisal of Potential Danger loop of Fig. 2 connects with the HPA axis, which has both input from and output to the Appraisal loop. Within the Appraisal loop there are receptors for CRH and GC, denoted by the respective circles in white rectangles, by which HPA can modulate it; CHR also acts as a neurotransmitter within the Appraisal loop. Finally, the Appraisal loop affects Autonomic Regulation, by which the myelinated vagus modulates the bronchi and heart. AM, amygdala; BNST, bed nucleus of the stria terminalis; CRH, corticotropin-releasing hormone; GC, glucocorticoid; HPC, hippocampus; LC, locus coeruleus; MOPFC, medial prefrontal cortex and orbital prefrontal cortex; PVN, paraventricular nucleus. From "Adaptation to potential threat: the evolution, neurobiology, and psychopathology of the security motivation system," by E.Z. Woody and H. Szechtman, 2011, Neuroscience and Biobehavioral Reviews, 35(4), p. 1029. Copyright 2010 by Elsevier. Reprinted with permission from Elsevier.

a generative way and raises questions that have not been raised as clearly before. The advantages of the SMS theory as a working framework for understanding OCD are illustrated by the present section, as well as in section $\mathrm{V}$ to follow.

It is useful to highlight some of the most important implications of the theory that OCD is a dysfunction of the SMS, conceptualized as a specialized, but normal motivation system. First, that a special but normal motivation underlies OCD sheds light on some of its otherwise puzzling characteristics. For instance, the behavior of individuals with OCD appears strangely jarring to a naïve observer: On the one hand, individuals with OCD behave as if they are engaged in something very important, being so preoccupied with their "obsessions" and "compulsions" as to interfere with normal daily function. On the other hand, it is clear to everyone-including the individuals with OCD-that the extent of this preoccupation is unreasonable and disconnected from objective reality. One way to reconcile such jarring disconnection between knowing rationally that one's actions are exaggerated and yet feeling compelled to perform them is to ask whether a similar condition can occur normally, without psychopathology.

Indeed, this kind of disjuncture is not an unusual situation with the activation of motivations that are "special." In his 1966 A Textbook of Psychology, Donald Hebb highlighted the class of "special" motivations: motivations that are "necessary for survival" of the individual and the species (Hebb, 1966, p. 206). Hebb recognized five special motivations (hunger, pain, sex, maternal, and exploratory motivations), but to this list should be added security motivation because it, too, is a primal motivation vital for survival (Szechtman and Woody, 2004; Woody and Szechtman, 2005). Once a special motivation is activated, the urge to perform survival-related species-typical actions dominates all behavior and thoughts and this motivation cannot readily be stopped rationally; for instance, the thirst for water cannot be satisfied by suggestions that one is not or should not be thirsty (Denton et al., 1999; Woody and Szechtman, 2005). Hence, it is a normal property of special motivations to compel performance of survivalrelated actions, and objectively highly motivated performance at times may look excessive, even to the individual. Thus, the apparent disjoint between feeling compelled to engage in OCD behavior and knowing that such behavior could look excessive is expected because actions induced by special motivations are mainly driven by built-in imperatives of survival, not rationally derived thoughts.

This insight has important implications for OCD research. The internal computations of special motivation systems are not accessible to the individual; thus, much or even most of OCD patients' thoughts about their behavior are post hoc explanations or reasons, rather than the actual causes of the behavior. It follows that phenomenological reports of why patients with 
OCD think they behave as they do-how they attempt to explain their behavior to themselves and others-are very suspect as potential scientific explanations (Mercier and Sperber, 2017). Instead, OCD research needs to focus more on the underlying survival-related mechanisms of the SMS, which must largely be determined by examining basic security-related stimulus-response patterns and their neurobiological underpinnings. For these purposes, laboratory research with animals is probably essential.

A consideration of the particular types of survival problems handled by the SMS sheds further light on some other otherwise puzzling aspects of OCD. To a naïve observer, individuals with OCD can seem surprisingly preoccupied with subtle, indirect, uncertain cues suggesting hazardous possibilities that have not yet happened, may be quite unlikely, and might never happen at all. However, as outlined earlier, these are exactly the properties of the stimuli that the SMS, as a normal motivation system, evolved to detect and assess-namely, possible indicators of potential danger, rather than signs of manifest danger. In this regard, it is important to distinguish the SMS from what Ohman and Mineka (2001) called the "fear module," which responds to cues of manifest danger with fear-based escape and avoidance learning. The SMS is functionally distinct from the fear module in several important ways:

1) it is based on subtler, unconditioned stimuli suggesting hidden risk, typically in the absence of any signs of manifest danger; and 2) its central motive state is anxiety or wariness, rather than fear, involving activation of distinct brain regions (see Blanchard et al., 2011); and 3) its characteristic behavioral output typically involves probing the environment and gathering information, rather than avoidance. (Woody and Szechtman, 2011, p. 1021)

There are also other important implications of the hypothesis that underlying OCD is a special motivational system, one that in most individuals is adaptive in managing the detection of and response to potential danger. Because this is a normal system present and operating in all people, it is important to distinguish clearly which specific properties of this system are pathologic in OCD.

In particular, both activation of security motivation in response to cues for potential danger and subsequent engagement in protective and precautionary behaviors are normal functions of the SMS, not OCD pathology per se. To illustrate, consider research in nonclinical samples associating hormonal changes with putative OCD symptoms. During the luteal phase of the menstrual cycle, healthy women report an increase in "unusual" cleaning behaviors, including excessive cleaning and cleaning of things not typically cleaned (Dillon and
Brooks, 1992), and an increase in behaviors related to self-cleanliness, grooming, and protection from germs (Fleischman and Fessler, 2011). Similarly, in individuals not previously diagnosed with OCD, pregnancy and childbirth may be associated with patterns reminiscent of OCD symptoms, including intrusive thoughts about potential dangers to the infant, such as some kind of bad accident or exposure to contamination (Leckman et al., 1999; Abramowitz et al., 2007), and repeated checking on the baby even though they "knew everything was fine" (Leckman et al., 1999; Abramowitz et al., 2007). Although findings like these may be interesting, from the perspective of the SMS theory, they are not truly akin to OCD because they simply involve variants of normal SMS activation and the corresponding precautionary behavioral responses.

According to the SMS theory of OCD, the crucial fault in OCD lies not in the activation of security motivation and the resulting protective behaviors; rather, it lies in the termination or stopping process. More generally, well differentiated activation and termination dynamics are a recognized property of the regulatory networks governing motivational processes (Park and van Leeuwen, 2014). Looking toward the next section on psychopharmacology, the hypothesis that the crucial defect lies in stopping - that is, difficulty in terminating SMS activation-is an important clue in research on possible pharmacological treatments for OCD.

Although increases in the activation tendency of the SMS are not the cause of OCD, they could make preexisting OCD worse. This is because readier activation would yield more circumstances in which the basic stopping deficit would become evident. For example, hormonal changes may increase the frequency of activation of the SMS, which would make preexisting OCD more problematic. Along these lines, Guglielmi et al. (2014) found that among women diagnosed with OCD, $38 \%$ reported an exacerbation of existing symptoms during the week prior to menses and about $40 \%$ experienced a worsening of symptoms during pregnancy or shortly after childbirth. Looking again toward the next section, activation dynamics could be relevant for pharmacological research in a somewhat secondary way, because reducing the likelihood of SMS activation may preclude the underlying stopping deficit.

Also of importance is the hypothesis that the SMS is a network coordinating activity across multiple brain systems, with information flowing through feedback loops, which also involve autonomic regulation and the HPA axis (Woody and Szechtman, 2011). This framework highlights the functional integrity of information flow throughout the network and the prospects for enhancing or rehabilitating it in OCD. Because of the complexity of the system, there could be many factors that could influence the integrity of this information flow; however, because of the self-correcting qualities of the system's feedback loops, the effect of any one such 
influence might reasonably be expected to be somewhat limited.

Finally, to the extent that the present framework instantiates the $\mathrm{RDoC}$ approach to psychopathology, the resulting research trajectory should not simply come full circle, arriving back at the same DSM-style definition and conception of OCD to which the RDoC was supposed to provide an alternative. Ideally, research would focus instead on the various possible maladaptive variants of SMS functioning, rather than confining itself to the one classic, categorical diagnosis of OCD. Working out the implications of this broader conception is an important task for future research.

\section{Toward a New Perspective on Psychopharmacological Research on Obsessive-Compulsive Disorder}

\section{A. Introduction}

With the SMS theory as a guiding framework, we now turn to a consideration of alternative ways to conceptualize potential targets for pharmacological intervention in OCD. Our aim is both to systematize the major possible approaches and also to outline some new ideas for psychopharmacological research on OCD.

As reviewed in section III, an extensive literature has addressed a vast range of factors that may affect OCD behavior. The motivation underlying much of this research is likely the hope that somewhere among all of these findings there will eventually emerge the clues leading to a chemical agent with a specific, potent, corrective effect on OCD-a "magic bullet," so to speak.

Indeed, the term "magic bullet" has been used to describe pioneering pharmacological discoveries, such as Paul Ehrlich's development of compounds for the treatment of syphilis (Witkop, 1982; Bosch and Rosich, 2008). However, such approaches can work well mainly if the underlying cause is a bacteria, parasite, or virus that has a unique chemical signature, distinct from that of the host. Unfortunately, for psychiatric disorders like OCD, which are not caused by an infectious agent but instead reflect some disturbance in the dynamics of a normal brain network, it seems unlikely that the disturbed network would have a unique chemical signature, distinct from the normal one. This is because all brain systems are constructed from the same pool of elemental constituents; it is the pattern of connectivity that gives each network distinct functional properties.

Even without any specific infectious agent, a normal system can become "out of tune," so to speak, by which we mean that disharmony in the dynamics of the system may yield the patterns characteristic of psychiatric symptoms, as we have hypothesized in linking the SMS to OCD. In the absence of a specific malicious entity to target, the strategy for pharmacological intervention could focus instead on drugs to shift the dysfunctional system in the direction of its normal dynamics. We next examine some alternative approaches for conceptualizing how to accomplish such rebalancing.

\section{B. Enhancing Infrastructure to Boost Processing Power of the Brain}

Although the "magic bullet" approach prizes specificity (i.e., drugs that target only an invading "enemy" without damaging the host), an important alternative strategy is, by contrast, nonspecific. Here, rather than a direct effect on a faulty element itself, the aim of pharmacotherapy is a global enhancement of brain processing.

In contrast to the search for "magic bullets" to cure specific ills, there is the traditional quest to find the Elixir of Life, a "magical" substance or liquid that gives eternal life (https://en.wikipedia.org/wiki/Elixir_of_life). Rather than repairing specific damage previously suffered, such a potion would enhance overall bodily processes to make life enduring and resilient to any future harm. In more current terms, what such an elixir of life aims to boost is the infrastructure of a healthy bodythe panoply of biologic facilities necessary for the body to function well. Boosting the body's infrastructure better enables the body's various specialized systems, including those in the brain, to effectively carry out their operations.

This perspective may readily be applied to our central theme of OCD as a disturbance in the dynamics of the SMS. In particular, the SMS may become out of balance not only because of a specific fault within the system itself but also because of some unreliable or inadequate infrastructure support that is necessary for it to operate normally. By boosting infrastructure, an increased supply of needed inputs may allow the vulnerable neural constituents to perform more properly, thus shifting the operation of security motivation toward normal.

Boosting infrastructure is a nonspecific mode of therapeutic intervention because the increased supply of infrastructure facilities would not only aid a compromised SMS but would also be of potential value to all brain systems. Nonetheless, for brain systems already functioning optimally, an infrastructure boost may simply provide an unnecessary oversupply of supports without functional utility.

An interesting analogy for the logic of the proposed approach is interventions to regulate the economy. There are many tools to stimulate the economy, and these can be used successfully without identifying what the underlying problem is or directly fixing this problem. For instance, cutting taxes or lowering the interest rate may be effective even though neither the tax rate nor the interest rate is the underlying cause of the poorly functioning economy. Such interventions, which target the infrastructure of economic activity, may raise economic growth even though more specific core deficiencies have not been identified or directly corrected. 
To illustrate this approach of boosting the biologic infrastructure relevant to OCD, we next consider as examples three particular classes of possible intervention.

1. The Monoamine Systems. Perhaps surprisingly, some current treatments for OCD can be interpreted as in fact boosting infrastructure facilities that use serotonin and dopamine neurotransmission.

a. Serotonin. In a recent positron emission tomography study using $\left[{ }^{11} \mathrm{C}\right]-\alpha$-methyl-L-tryptophan as a tracer for serotonin synthesis, 10 of 16 patients with OCD benefitting from a 12-week course of either the SSRI sertraline or CBT treatment showed a "brain-wide, rather than localized, enhancement of central 5-HT synthesis capacity" (Lissemore et al., 2018), indicating that "increases in 5-HT synthesis capacity with effective treatment support a compensatory, rather than pathological, role of 5-HT neurotransmission in OCD" (Lissemore et al., 2018). In other words, rather than being specific to any particular brain region, the neurobiological shift associated with improvement was available to the entire brain, which is consistent with the notion of boosting an infrastructure facility. Another way in which brain serotonin systems demonstrate the hallmark of an infrastructure facility is that a set of neurons concentrated in a relatively small region of the brain, the nuclei raphes lineares, has a global impact by innervating almost the entire central nervous system (Jacobs and Azmitia, 1992; Charnay and Léger, 2010; Fidalgo et al., 2013). Also supporting the conception of an infrastructure boost as the underlying therapeutic mechanism is the evidence that treatments that target serotonin neurotransmission are effective not only for OCD but also for a number of psychiatric disorders (Katzman et al., 2014). Moreover, it had been noted that "serotonin is an enigma. It is at once implicated in virtually everything, but responsible for nothing" (Jacobs and Fornal, 1995, p. 461). However, rather than being enigmatic, this characterization of serotonin is an apt description of an infrastructure facility.

How might the infrastructure support provided by serotonin signal transduction benefit an out-of-tune SMS? One reasonable possibility is that the monoamine systems (serotonin, dopamine and norepinephrine) constitute a network managing brain "arousal" (Pfaff, 2006), where arousal denotes the category of neurobiological processes necessary for neural circuits to perform their specialized functions, both input and output. The arousal infrastructure encompasses complex molecular, biochemical, endocrine, and genetic mechanisms and includes the provision of energy and nutrients and the removal of waste products, all of which are affected by serotonin (Azmitia, 2001; Charnay and Léger, 2010; Fidalgo et al., 2013). In summary, a boost in the management of arousal, as provided by a regulated injection of serotonin activity, might plausibly shift out-of-tune SMS dynamics toward normal operation.

b. Dopamine. Not only serotonin but also dopamine systems have a neuroanatomy indicative of an infrastructure facility (a circumscribed location of neurons yet relatively widespread innervation). As noted previously, drugs targeting dopamine neurotransmission may augment effectiveness of SSRI treatment in OCD (section II.B.2). In accordance with the perspective just mentioned, this clinical observation suggests that another factor affecting arousal is modulation of dopamine activity. It seems likely that serotonin and dopamine play distinct but complementary regulatory roles in managing arousal. In particular, dopamine may act to instigate arousal and approach, whereas serotonin may promote deactivation of arousal, with the two systems balancing in a coordinated manner (Soubrie, 1986; Kapur and Remington, 1996; Szechtman and Woody, 2004; Alex and Pehek, 2007; Di Giovanni et al., 2008). Studies using animal models of OCD lend support to this notion (Alkhatib et al., 2013; Tucci et al., 2014a,b; Johnson and Szechtman, 2016). In summary, an appropriate level of arousal, provided by interventions that rebalance dopamine and serotonin activity, may shift out-of-tune SMS dynamics toward normal operation.

c. Serotonin or Dopamine Boost? Whether out-oftune SMS dynamics are normalized better with a dopamine or a serotonin intervention (or both) could hinge on the profile of OCD symptoms (Fontenelle et al., 2005; Mataix-Cols et al., 2005; van den Heuvel et al., 2009; Matsunaga et al., 2010; Torresan et al., 2013), a hypothesis readily researched using different animal models of OCD in parallel. It had been suggested that because different animal models of OCD likely capture different parts of OCD heterogeneity, it is possible to investigate whether a particular treatment regimen is best suited for a particular OCD presentation by testing the effects of that same treatment in more than one animal model. As reviewed in section II.C.2, Winter and colleagues employed such a paradigm to examine treatment effects of DBS, employing both the QS (Szechtman et al., 1998) and the SA (Joel, 2006) rat models of OCD (Winter et al., 2008; Klavir et al., 2009, 2011; Djodari-Irani et al., 2011). Because treatment effectiveness diverged in the two models at one of the brain sites targeted by DBS, they suggested that the DBS therapeutic strategy for patients should differ, depending on whether the dopamine-serotonin balance in the patient is tilted in favor of dopamine (as in the QS model) or tilted toward serotonin (as in the SA model) (Szechtman et al., 2017).

In addition to examining the effects of the same treatment in different animal models, another approach would be to make use of individual differences among animals in performance of model OCD behavior. For example, it could be that boosting serotonin is beneficial at the low end of performance intensity, whereas boosting dopamine is beneficial at the high end. Such 
findings in animal models that are based in particular on special motivations may provide guidance on whether to boost serotonin or dopamine infrastructure facilities to shift out-of-tune SMS dynamics toward normal operation, ameliorating OCD.

d. Norepinephrine Pathways. Although OCD pharmacotherapy in the clinic does not include agents that specifically target norepinephrine, norepinephrine pathways play a critical role in the brain arousal network (Pfaff, 2006); hence, boosting norepinephrine activity may have therapeutic value in shifting out-of-tune SMS dynamics toward normal operation. A rational approach to investigating this possibility could consider the different norepinephrine receptors, especially the $\alpha_{1 / 2}$ and $\beta_{1 / 2}$ receptors that manage arousal and stress responding (Uys et al., 2017a). Activation and antagonism of $\alpha_{2}$ receptors inhibit and enhance rotational behavior in rats, respectively (Mavridis et al., 1991). However, more subtype-selective action at $\alpha_{2 \mathrm{c}}$ receptors, for example, may be particularly of benefit to enhance the appraisal aspects of SMS activity (Uys et al., $2017 \mathrm{a})$, given that the $\alpha_{2 \mathrm{c}}$ antagonist, ORM-10921 [(1R*,12bR*)-(-)-1,3,4,6,7,12b-Hexahydro-1-methoxymethyl1-methyl-2H-benzofuro[2,3- $\alpha$ ]quinolizine hydrochloride], is procognitive (Uys et al., 2016) and antidepressant (Uys et al., 2017b) in translational animal models, thus warranting study in animal models of OCD for potential benefit in OCD itself.

More generally, a rational approach to identify compounds that would be of value in boosting monoamine activity for OCD treatment should entail basic science research to identify the particular jobs that serotonin, dopamine, and norepinephrine perform in the management of arousal (Pfaff, 2006).

\section{Circadian Rhythm Facility.}

a. Importance of Circadian Rhythms for Optimizing Brain Resources. As noted earlier in section II.C.2, the therapeutic effect of DBS in OCD may involve a reset of loosely coupled oscillators leading to enhanced information flow in BG-TH-CTX circuits (Parent and Hazrati, 1995a,b; Montgomery, 2007, 2016, 2017; Montgomery and Gale, 2008; Montgomery and $\mathrm{He}, 2016)$. However, information flow in neural networks is not the only process that involves oscillatory activity. Throughout the body, a large number of biochemical processes underlying physiology and behavior show rhythmic cycles of activity, many recurring at approximately 24-hour intervals ("circadian" rhythms) (Stratmann and Schibler, 2006; Cermakian and Boivin, 2009; Dibner et al., 2010; Konturek et al., 2011; Piggins and Guilding, 2011; Logan and Sarkar, 2012; Brown and Azzi, 2013). The near-24-hour oscillations in biochemical processes are regulated by timing signals from biologic clock cells ("circadian oscillators") located in the brain and in peripheral organs and tissues (Mistlberger and Antle, 2011). Outputs of different autonomous clocks can become coupled, providing a synchronized temporal framework for the various physiologic and behavioral processes. One major mechanism for coordinating the timing of the various oscillators in the brain and body is the group of clock cells located in the suprachiasmatic nucleus (SCN) of the hypothalamus; these SCN clock cells function as a "master pacemaker" that maintains control over and synchronizes the "slave" circadian oscillators outside the SCN, which control their own circadian phenomena (Bernard et al., 2007; Mistlberger and Antle, 2011). In addition to internal synchrony, the SCN provides for external synchrony in that the timing of its circadian rhythm can be reset and entrained by light/dark cycles associated with the solar day. External synchrony serves to align internal and external times, providing for internal circadian physiologic and behavioral events (e.g., sleep/wake cycle) to occur at appropriate times of the day (Mistlberger et al., 2000). External synchrony can be implemented through adjustment of two clock parameters: the clock period (the duration of one complete cycle) and the clock phase (the position within a cycle). A stimulus is designated a zeitgeber ("time giver") if it controls these clock parameters, entraining the circadian oscillator.

Because the outputs of biologic clocks regulate a broad range of biochemical processes underlying physiology and behavior, the network of such clocks can be conceptualized as an infrastructure facility. Accordingly, the job of this infrastructure is to operate the biologic clocking machinery to output rhythmical timing signals that not only produce oscillation in various physiologic and behavioral processes but also impose synchronous activity.

How could a boost or intercession on the circadian rhythm infrastructure shift the operation of an out-oftune SMS toward normal and be of therapeutic value in OCD? As argued before, the elixir of life approach supposes that the SMS is out-of-tune at least in part because it is not supplied reliably with some infrastructure input or inputs needed to operate properly. Considering that the circadian rhythm facility synchronizes activity of many biochemical processes, this coordination produces amplified output, thus making available a greater supply of inputs to shift the operation of an out-of-tune SMS toward normal function.

There is much neuroscience knowledge on the timekeeping mechanisms in the body and the molecular and neural mechanisms by which the SCN entrains and synchronizes central and peripheral circadian oscillators (Mistlberger et al., 2000; Bernard et al., 2007; Cermakian and Boivin, 2009; Dibner et al., 2010; Kalsbeek et al., 2011; Mistlberger and Antle, 2011; Piggins and Guilding, 2011; Morris et al., 2012; Dallmann et al., 2014; Hastings et al., 2014, 2018). This knowledge points toward possible targets for a judicious intercession on the circadian rhythm infrastructure.

Circadian oscillators appear to have been selected by natural selection as an adaptive mechanism to respond 
in a "24-hour world" to environmental change by providing the "capacity to anticipate predictable phenomena. By adjusting metabolism, physiology and behaviour in advance of these changes in light and dark, and hot and cold, the organism is ready for the challenges of the coming day or night" (Rosato and Kyriacou, 2011, p. 72). In other words, circadian rhythms are a temporal order mechanism to optimize the supply of resources for usage in an upcoming anticipated physiologic or behavioral task (Hastings et al., 2003; Dallmann et al., 2014). It follows that when the anticipated task is forced to a new time, the timing of the relevant circadian rhythm ought to shift accordingly (Morris et al., 2012; Arendt, 2018). Indeed, certain zeitgebers are effective stimuli in regulating circadian rhythms. Light pulses are well recognized photic zeitgebers but equally important are nonphotic ones, each with distinct mechanisms of action (Mrosovsky, 1996; Buijs et al., 2006; Mistlberger and Antle, 2011). Of relevance to OCD, here we highlight behavioral zeitgebers to boost the circadian infrastructure, with mechanisms of action open for pharmacological intervention.

b. Pathways by Which Nonphotic Zeitgebers May Boost Circadian Infrastructure and Thus Ameliorate Obsessive-Compulsive Disorder. When daily life is stable and consistent, circadian cycles can operate at peak efficiency supplying maximum bodily resources just when they are needed for the different upcoming tasks. However, if there is a change in the timing of vital events, for instance, when switching to night shift work, one or more of the circadian rhythms would be reset (Morris et al., 2012). But even less dramatic happenings can reset the master SCN circadian pacemaker. One such event is a mild perturbation of the animal during its rest (sleep) period, such as cleaning its home cage or gentle "handling" of the animal. Such a disturbance of the animal can shift the circadian clock either forward or backward, depending on the time frame of this perturbation: a disturbance during the mid-to-late phase of the sleep phase of the rest-activity cycle induces a phase-advance shift, and a phase-delay shift is induced by a perturbation during the active phase of the restactivity cycle or during the beginning of the rest (sleep) phase (Mistlberger and Antle, 2011). Extensive analysis of the neural and molecular pathways of this nonphotic gateway to the circadian clock, and of similar zeitgebers, has provided potential targets for pharmacological intervention with clock-resetting compounds ("chronobiotics") as a boost to the circadian infrastructure and the realignment of circadian rhythms (Mistlberger et al., 2000; Mistlberger and Antle, 2011). Interestingly, there is much convergent evidence that nonphotic modulation of the SCN pacemaker is through serotonergic innervation from the raphe nucleus and melatonin receptors in the SCN (Reppert et al., 1994; Glass et al., 2003; Honma and Honma, 2009; Mistlberger and Antle, 2011).
These nonphotic zeitgebers (disturbing the animal through cleaning its home cage or handling by the human caretaker) have been viewed as stimulating "arousal" (Mistlberger and Antle, 2011). However, these procedures no doubt activate the SMS, given that they invoke cues of potential danger (signs of home breach, "capture" by a possible predator). Hence, most likely, activation of security motivation is a nonphotic gateway to the SCN circadian rhythm.

Thus, at least for some individuals, perturbations of circadian rhythms and OCD tendencies may be interrelated problems (Nota et al., 2015). Indeed, there is emerging clinical evidence that the chronobiotic agomelatine may be effective in patients with OCD (da Rocha and Correa, 2011; Fornaro, 2011; Lange et al., 2012; Tzavellas et al., 2014; Perugi et al., 2015). As a chronobiotic, agomelatine re-entrains desynchronized biologic rhythms by acting as a melatonin MT1 and MT2 receptor agonist and serotonin 5-HT2C receptor antagonist (de Bodinat et al., 2010; Guardiola-Lemaitre et al., 2014; MacIsaac et al., 2014; Yang et al., 2016).

A case report in which agomelatine was given as monotherapy to a 22-year-old woman with treatmentresistant OCD (De Berardis et al., 2012) may be of particular interest and instructive regarding how a boost to circadian infrastructure may ameliorate OCD. This individual had a YBOCS score of 30. Her symptoms included severe compulsive washing. Of note, she reported that her concerns of "possible contamination of her bedsheets" led to "difficulty sleeping." She had discontinued her prior (ineffective) medications (fluvoxamine, clomipramine, and venlafaxine) for at least 3 months and was prescribed agomelatine to be taken at bedtime. After 2 weeks, there was a minimal reduction in OCD symptoms (YBOCS score of 28) but the individual continued taking the drug because "she noticed a significant improvement in sleep quality." Her OCD improved with continued treatment and remitted fully by 8 weeks (YBOCS score of 6 ). She continued taking agomelatine and had no OCD symptoms (YBOCS score of 3) at the last examination months later (De Berardis et al., 2012).

As is evident, a noticeable improvement in the patient's sleep preceded marked improvement in her OCD, which fully remitted eventually. Presumably, the bedtime schedule of taking agomelatine re-entrained and synchronized circadian rhythms, shifting SMS activity toward normal operation, and thus away from symptoms of OCD. One possible mechanism underlying this effect may involve the circadian expression of MT1/2 and 5-HT2C receptors that allows phasic binding of agomelatine to its target receptors (Harvey and Slabbert, 2014).

Note that the timing of chronobiotic treatment (over the course of the day) may be important, depending on whether a phase advance or phase delay is more appropriate or whether the treatment affects other 
biochemical processes; for instance, all major neurotransmitter systems have circadian rhythms (Dallmann et al., 2014). Furthermore, it is worth mentioning that given that circadian rhythms anticipate predictable phenomena by cues that reflect "time interval, time of day or time of year, which is measured by the biological clock(s)" (Honma and Honma, 2009, p. 1707), there are additional, nondrug interventions for improving circadian rhythms by adhering to a consistent schedule, including exercise (Cotman and Berchtold, 2002) and regular meal times (Honma and Honma, 2009; Mistlberger and Antle, 2011); hence, these too should be of benefit in treating OCD. Indeed, there are reports of benefits of exercise in OCD (Rector et al., 2015; Abrantes et al., 2017, 2019), although utility of regular meal times has not been examined. More generally, animal models provide important opportunities to examine how circadian rhythm regulation relates to the operation of the SMS and thus the treatment of OCD.

3. Nicotinamide Adenine Dinucleotide Metabolism Infrastructure.

a. Importance of Nicotinamide Adenine Dinucleotide in Living Cells. Whereas the monoamine and circadian rhythm infrastructure facilities operate in whole or in part as distinct neural systems with their own neuroanatomical pathways, the nicotinamide adenine dinucleotide (NAD) metabolism infrastructure operates primarily within the cell itself. It supports a variety of cellular processes, including cellular metabolism and energy production, redox homeostasis, mitochondrial functions, antioxidation/generation of oxidative stress, $\mathrm{Ca}^{2+}$ mobilization, gene transcription, immunologic functions, aging, and cell death (Belenky et al., 2007; Ying, 2006, 2008). Involvement in so many biologic activities suggests that a judicious boost of this infrastructure could shift an out-of-tune SMS toward normal operation and be of benefit in OCD.

b. Redox Reactions. The molecule NAD is present in all living cells where one of its infrastructure functions is to serve as a coenzyme in reduction-oxidation (redox) reactions. NAD exists in two forms in the cell: as an oxidizing agent $\left(\mathrm{NAD}^{+}\right)$accepting electrons from other molecules and as a reducing agent $(\mathrm{NADH})$ donating electrons. NAD is also converted to NADP and serves in redox reactions in its phosphorylated $\left(\mathrm{NADP}^{+}\right)$and reduced (NADPH) forms. The chemistry of NADP is similar to that of NAD, but NADP is used predominantly as a coenzyme in anabolic biochemical transformations, whereas the $\mathrm{NAD}^{+} / \mathrm{NADH}$ pair is more important as a coenzyme for hydride-transfer enzymes in catabolic reactions. Because these redox reactions influence many fundamental processes in every living cell, it had been suggested that "nicotine adenine dinucleotides [be conceptualized] as the 'redox currency' of the cell" (Fessel and Oldham, 2018, p. 201).

c. Substrate for Enzymes. In addition to its coenzyme role in redox reactions, NAD is also a substrate for enzymes that cleave $\mathrm{NAD}^{+}$to produce nicotinamide and an ADP-ribosyl product. These enzymes fall into three classes, collectively referred to as $\mathrm{NAD}^{+}$-consuming enzymes (Belenky et al., 2007). The three classes of $\mathrm{NAD}^{+}$ consumers are 1) ADP-ribose transferases or the more numerous poly(ADP-ribose) polymerases (PARPs), 2) cADP-ribose synthases, and 3) sirtuins (type III protein lysine deacetylases) (Belenky et al., 2007). The ADPribose reaction products from $\mathrm{NAD}^{+}$catabolism with ADP-ribose transferases and PARPS are involved in DNA-damage responses, epigenetic modification, transcription, chromosome segregation, programmed cell death, and repair signaling, whereas cADP-ribose synthase enzymes (also known as lymphocyte antigens CD38 and CD157) produce and hydrolyze the $\mathrm{Ca}^{2+}$. mobilizing second-messenger cADP-ribose from $\mathrm{NAD}^{+}$. Finally, the third class of $\mathrm{NAD}^{+}$-consuming enzymes, sirtuins (also termed type III histone deacetylases), cleave NAD in the process of removing the acetyl group from acetylated lysine, yielding a deacetylated protein lysine and an acetylated ADP-ribose plus nicotinamide (Belenky et al., 2007). The importance of sirtuins lies in regulating "complexes that remodel chromatin to repress transcription and recombination in a manner that depends on reversing acetyl modifications on histone H3 and histone H4" (Belenky et al., 2007, p. 14).

d. Biosynthesis and Strategies to Boost Nicotinamide Adenine Dinucleotide. Mammals have a large daily requirement for NAD due to substantial flux through the $\mathrm{NAD}^{+}$-consuming pathways (Belenky et al., 2007; Fessel and Oldham, 2018), suggesting the presence of mechanisms for regulating the abundance of NAD. There are two routes for biosynthesis of NAD. One is through the salvage pathway using the vitamin $\mathrm{B}_{3}$ (niacin) preformed precursors of $\mathrm{NAD}^{+}$: nicotinic acid, nicotinamide, and nicotinamide riboside (Fessel and Oldham, 2018). Another is de novo from degradation of tryptophan to quinolinic acid in the kynurenine pathway and subsequent conversion of quinolinic acid to nicotinic acid mononucleotide, which enters the salvage pathway for NAD synthesis. The de novo route is operational when the dietary supply of tryptophan is high because downstream metabolites of tryptophan in the kynurenine pathway are typically diverted to other branches, limiting the supply of quinolinic acid for NAD biosynthesis (Fessel and Oldham, 2018). The salvage pathway is the primary route for effective NAD maintenance, recycling the preformed compounds released by reactions that break down the structure of NAD, and by entering these components into the salvage pathway from the diet (Fessel and Oldham, 2018).

Hence, a boost of the NAD infrastructure is plausible by raising salvage of components from NAD breakdown and enhancing precursor uptake from the extracellular environment. One straightforward strategy is through consumption of foods rich in the precursors for NAD synthesis and/or through exogenous supplementation of these precursors. In a recent review, the authors 
note that "nicotinamide riboside appears to be effective for increasing intracellular NAD in murine models and likely in humans as well when supplemented in the diet" (Fessel and Oldham, 2018, p. 200). Another strategy for modulation of NAD metabolism is metformin, the representative drug of the biguanides, as this drug appears to exert beneficial effects by driving the NAD/NADH ratio by several different mechanisms of action (Fessel and Oldham, 2018). Yet other means to boost NAD infrastructure include the development of direct activators of sirtuins, inhibitors of NADPH oxidase isoforms, and next-generation inhibitors of PARP family enzymes in an effort to pharmacologically mimic the life-extending effects of caloric restriction (Belenky et al., 2007; Fessel and Oldham, 2018). Also of potential interest are pharmacological modulators of the NAD-electron transfer system in mitochondria. For instance, methylene blue, by acting as an electron acceptor/donor, can improve mitochondrial function (Delport et al., 2017, 2018). Other pharmacological approaches worthy of investigation that could boost the NAD pool include antioxidants, such as NAC, and modulators of TRYCAT metabolism, such as allopurinol (a tryptophan 2,3-dioxygenase inhibitor) and RO-61848 (a kynurenine 3-monoxygenase inhibitor).

4. Conclusion. We have reviewed three major types of infrastructure facilities: the monoamine systems, circadian rhythms, and NAD metabolism. These were chosen because they seemed particularly relevant to OCD and amenable to a boost in performance by interventions. However, they are not necessarily exhaustive, and the many factors reviewed in section III (e.g., microbiota, neurosteroids and neuropeptides, hormones, and the immune system) may suggest other infrastructure facilities that could possibly benefit from targeted interventions. There may be further possibilities as well, for example, the trace-amine rheostat-like system (Gainetdinov et al., 2018).

The perspective taken in this section is that, irrespective of what the primary cause or causes of OCD may be, boosting infrastructure facilities may help shift the SMS toward more adaptive patterns of responding. This idea is somewhat akin to the concept of disease tolerance, but without necessarily implying the presence of a pathogen. Disease tolerance is a defensive strategy in which, the organism, rather than directly attacking the disease's primary cause, marshals resources to ameliorate the deleterious effects that the presence of this primary cause would otherwise give rise to (Medzhitov et al., 2012).

An alternative conception of the role of infrastructure would be to entertain the possibility that, at least in some cases, poor infrastructure facilities are the primary cause of OCD. However, faulty infrastructure would be likely to affect multiple brain networks, which might reasonably be expected to yield a broader picture of psychiatric disorders, rather than OCD alone. Hence, an interesting hypothesis is that infrastructure deficits may account for at least some cases of OCD that are comorbid with other psychiatric disorders (du Toit et al., 2001; Fireman et al., 2001; Tükel et al., 2002; Szechtman and Woody, 2006).

\section{Facilitating Relearning to Promote Neural Plasticity}

Like other special motivation systems, the SMS consists of genetically "prebuilt" or "hardwired" components that evolved to process and respond to a particular class of survival-related challenges. Nonetheless, even such systems require learning to calibrate their operation, so that it is fine-tuned to the individual's particular physical and cultural circumstances (Woody and Szechtman, 2011). Although early life may be especially important for such calibration processes (Gluckman et al., 2005), recalibration most likely continues to some degree across the lifespan (HahnHolbrook et al., 2011; Lienard, 2011).

Particularly impressive evidence indicating that openness to recalibration in the SMS extends into adulthood is the effectiveness of psychotherapy for OCD, as reviewed in section II.D. Although the specific mechanisms underlying the effectiveness of psychotherapy have not yet been identified, the intervention process crucially involves acquisition of new information and reorganization of the knowledge network used by the SMS - that is, a process of learning or relearning.

However, as with other psychiatric disorders, there is a high rate of OCD patients in psychologic treatment who find themselves unable to complete the treatment and thus reap its benefits. For example, the nonadherence rate for ERP for OCD is about $20 \%$ for controlled clinical studies (Ong et al., 2016) and may be much higher in naturalistic studies that more closely approximate real-world clinical settings (Santana and Fontenelle, 2011). In a review of predictors of treatment nonadherence, Santana and Fontenelle (2011) found that the most important factor appeared to be the patients' expectations and beliefs about the disorder and the treatment that did not appear to correspond to therapy. We believe that the SMS theory of OCD may have a valuable role in this regard, because it offers a rationale that explicitly connects OCD with normal, adaptive behavior patterns common to all people, thus obviating the commonsense view of OCD behavior as purposeless or senseless.

Another potentially important route to improving the success of psychotherapy for OCD may be the concurrent use of drugs that are cognitive enhancers, which facilitate new learning through mechanisms such as boosting neural plasticity. Rather than acting directly to dampen symptoms like the drugs reviewed in section II.B, such cognitive enhancers would work by helping patients become more open to alternative ways to perceive and behave with regard to potential danger, 
thus fostering the learning that can shift patients toward more adaptive operation of the SMS.

Consistent with this possibility, several studies recently examined the use of drugs that are cognitive enhancers, such as D-cycloserine, yohimbine, and glucocorticoids, to augment the effectiveness of ERP (McGuire et al., 2014, 2017; Sulkowski et al., 2014). Some results with OCD are promising (de Leeuw et al., 2017); however, meta-analyses indicate that more research is required to clarify the role of therapy characteristics in drug-augmented outcomes (Mataix-Cols et al., 2017; McGuire et al., 2017). More generally, a diverse range of compounds have been identified as possible modulators of learning in psychiatry (Singewald et al., 2015), and it is possible that some of these drugs could accelerate learning processes elicited by psychotherapy for OCD.

The SMS theory of OCD offers some potentially useful distinctions in the kinds of relearning in psychotherapy that might be fostered by cognitive enhancers. Some kinds of learning may principally affect the input side of the SMS - that is, the appraisal of potential danger. For example, it is likely that ERP reduces the OCD patient's sensitivity to particular cues of potential danger of concern to the individual, making the SMS less readily activated and thus bypassing the main underlying dysfunction in stopping (Woody and Szechtman, 2005). Thus, one useful effect of cognitive enhancers would be to promote this desensitization process. In contrast, other kinds of relearning in psychotherapy may principally affect the output side of the SMS-that is, the capacity of engagement in preventative behavior to provide negative feedback to shut down activation of the SMS. Fostering learning on these output and feedback functions of the SMS is a possibility for cognitive enhancers (or other types of compounds) that may be quite distinct from promoting desensitization.

Such learning on the output side of the SMS is not typically a focus of CBT for OCD. For instance, in ERP the patient's relearning is guided by instructions not to engage in security-related responses when faced with cues for potential danger (i.e., "response prevention"). From the vantage point of the SMS theory of OCD, this strong de-emphasis on behavioral output is a clear deviation from the normal operation of the SMS. Normally, activation of the SMS by cues for potential danger leads to the performance of security-related behaviors, and this performance is crucial in affording the sense of task completion to shut down the system. A closer approximation to normal functioning of the SMS would be for OCD patients to learn security-related behaviors that are more moderate but come to be more strongly associated with yedasentience, or the feeling of task completion. This is a challenge for which cognitive enhancers, or other types of substances, could be especially helpful. With regard to other types of substances, compounds that enhance the negative feedback signal from motor action or the phenomenological experience of that feedback (yedasentience) would be worthy to assess for their therapeutic usefulness. One type of such compounds of potential clinical value is likely derivatives of cannabis, given the subjective experience of feelings of knowing or insight often reported with marijuana use (http://marijuana-uses.com/mr-x/; Tart, 1970).

Also of possible relevance, psychedelics were recently proposed as potential agents to foster relearning to overcome well engrained patterns (Carhart-Harris and Friston, 2019). As with the other drugs mentioned in this section, their use crucially must be accompanied by an appropriate framework for relearning, such as psychotherapy. Drugs to foster relearning would not be expected to be ameliorative in and of themselves.

In addition to the foregoing types of drugs, other important possibilities for interventions that might increase plasticity and foster relearning include immune modulation (Yirmiya and Goshen, 2011), glutamate drugs, and rTMS. In researching these alternative augmentation methods, it may be useful to consider the particular kind of learning that is being promoted, along the lines we have described.

\section{Fixing a Weak Link: Targeting Specific Pathways of the Security Motivation System Network}

The SMS theory of OCD hypothesizes specifically that it is the system-termination or stopping dynamics that are the crucial defect in OCD. Thus, a particularly promising place to look for treatment possibilities may be drugs (and possibly other interventions) that enhance or otherwise modulate the negative-feedback loop, associated with yedasentience, that plays a crucial role in shutting down SMS activation. However, for this purpose, it is essential to perform basic research on the underlying functioning of the SMS to understand the neurobiology of the specific fault that such interventions would affect.

\section{Motivational Reconceptualization of Animal} Models of Obsessive-Compulsive Disorders. A profound heuristic advantage emanates from framing OCD as a dysfunction of a normal "special" motivation, the SMS: A motivational framework summonses the vast behavioral neuroscience literature on motivation to the study of OCD. In so doing, it opens up the range of pertinent research questions that can be asked of OCD, provides exemplars of motivation paradigms that can be applied to investigate OCD, and illuminates research methods for studying motivation that can be used to study OCD.

Situating this research within a motivational framework leads naturally to a crucial question: How does motivated activity normally terminate? A shut down of motivation is not a passive event but an active neurobiological process with its own specific mechanisms. This has been established most clearly in the case of hunger motivation, where a number of factors have been identified that act at various brain sites to 
terminate eating (Woods, 2004; Gruninger et al., 2007; Smith, 2009; Lutz, 2011). Effects of these terminating signals are not confined to stopping the motor acts of eating - they include inhibition of the motivation itself and suppression of the reappearance of hunger motivation for an extended period of time (Teitelbaum, 1955; Toates, 1981, 1986; Collier and Johnson, 2004; Moran, 2004; Woods, 2004).

In engineering terms, motivation is modeled as a controller, with a negative feedback that functions to terminate the activity of the controller, shutting it down (Toates, 1986). There are different schemata of how various components of the controller are arranged; for motivational systems, most models take account of factors that are external and internal to the organism and include a negative feedback component that would terminate the activity of the controller once it has been engaged in producing the relevant output for some period of time (Toates, 1981). Although the negative feedback component in hunger motivation is often conceptualized simply as "satiety," it is important to note that the neurobiological mechanisms of this negative feedback satiety component are complex, in that there are parts that provide immediate and short-acting stop signals and other parts that supply signals for longer-term suppression of the motivation (Teitelbaum, 1955; Smith, 1996; Collier and Johnson, 2004; Moran, 2004; Sclafani and Ackroff, 2004; Strubbe and Woods, 2004; Woods, 2004).

By framing OCD within a motivational perspective, the schema of security motivation in Fig. 1 makes it intuitively cogent that with a faulty negative feedback, motivated activity would not be stopped and securityrelated behaviors would continue unabated. It is this perseveration of species-typical security-related behaviors such as washing and checking that provides for the presentation of OCD compulsions, as discussed earlier (section IV.B). Importantly, the schema in Fig. 1 and the proposed circuit diagrams shown in Figs. 2 and 3 offer a direction for research into the neurobiological mechanisms of the negative feedback stop signals, and they thus identify the neurobiological fault proposed to underlie OCD.

It is important, however, to distinguish the proposed mechanism from other types of "stopping" mechanisms in the literature (Aron, 2009). The specific stop mechanism we implicate in OCD pathology is the negative feedback signal that normally terminates security motivation (Szechtman and Woody, 2004; Woody and Szechtman, 2005, 2011). This mechanism differs from the type of stop deficit that some other investigators have posited may underlie OCD (Chamberlain et al., 2005, 2007; Penadés et al., 2007; Robbins, 2007; Chambers et al., 2009) and impulsivity (Winstanley et al., 2004b, 2005; Robbins, 2007; Eagle et al., 2009, 2011; Zeeb et al., 2010; Dalley et al., 2011). According to this contrasting view, what is impaired in OCD is the capacity for "behavioral inhibition," a type of frontoexecutive control over motor responding. Different components of this inhibitory, cognitive control process may be evaluated by tests such as the Stop-Signal Inhibition Task, the Go/No-Go Task, the Delay-Aversion/ Delay-Discounting Task, and the Five-Choice Serial Reaction Time Task (Winstanley et al., 2004a; Robbins, 2007; Groman et al., 2009; Verbruggen and Logan, 2009; Beste et al., 2010; Eagle and Baunez, 2010; Dalley et al., 2011). The hypothesis is that the underlying cause of OCD may be some deficit in the normal frontal mechanisms for withholding intended or canceling ongoing motor actions (Eagle and Baunez, 2010; Dalley et al., 2011). In contrast, our concept of the stop signal that fails in OCD is closely akin to the motivational construct of "satiety," and it is rooted in the theoretical understanding of how a course of behavior, once aroused by a particular motivation, comes to its normal termination upon goal attainment.

It is worth highlighting that probing for the neurobiological fault in negative feedback as the mechanism of OCD can be investigated in animal studies. For instance, it has been shown that the negative feedback "satiety" signal is abnormally short in the QS rat model of OCD (Szechtman et al., 1998; Dvorkin et al., 2006, 2010), making this a suitable preparation to investigate the neurobiology of the negative feedback that is faulty and insufficient. As an interesting side note, it may be worth noting that the quest to identify a negative feedback signal followed from a re-reframing of "compulsive checking" into a security motivation framework and that the methods to identify whether a negative framework exists were adapted from the literature on hunger motivation (Dvorkin et al., 2006).

Yet another way to probe for the neurobiological fault in OCD is to take advantage of the spontaneous readiness with which animals exhibit other special motivations and use that special motivation as a preparation to investigate neurobiology and the negative feedback mechanisms. For instance, maternal motivation is a special motivation that has interesting properties shared with SMS. In many animals, one aspect of maternal motivation is building a maternal nest before birth to house the newborn. Nest-building behavior has the flavor of compulsive behavior in that animals appear to be engaged intensely in bouts of activity composed of episodes of nest material collection, transporting it to the nest site, and nest construction; this activity is vigorous and persistent but stops after many repetitions of such bouts, presumably because the animal deems that the nest is finally "finished," which raises the question: how did the animal decide that it was done? In other words, what is the negative feedback signal that terminates nest-building activity? Using maternal nest building in the rabbit, Hoffman and Rueda Morales $(2009,2012)$ proposed that identifying the neural mechanisms that determine "how much nest 
is enough" would be informative for understanding the nature of the "stopping defect" in OCD and the brain regions modulated by negative feedback signals (Cano-Ramírez and Hoffman, 2017, 2018). Other investigators have proposed that variations in nest size observed in mice may be a useful marker to study how OCD presentation is affected by a range of factors (Greene-Schloesser et al., 2011; Mitra et al., 2016, 2017; Wolmarans et al., 2016; 2017), although the contribution of the negative feedback signal to these effects has not yet been explored.

2. Neurochemistry of the Negative Feedback "Satiety" Signal(s). Based on findings from other special motivations, the neurochemistry of SMS negative feedback is likely multifaceted, rather than consisting of a signal with a single neurochemical identity. For instance, termination of eating and hunger motivation is subject to control by a constellation and interaction of sensory and reafferent neural signals from body parts contacted by food, together with the neuropeptides and hormones stimulated by food ingestion (Teitelbaum, 1955; Smith, 1996, 2009; Moran, 2004; Sclafani and Ackroff, 2004; Strubbe and Woods, 2004; Clifton, 2017). Arrest of sexual motivation by sexual satiety is similarly mediated by numerous factors (Fernández-Guasti and RodríguezManzo, 2003; Phillips-Farfán and Fernández-Guasti, 2009; Barrozo et al., 2010a). Likewise, we should expect that termination of security-related behaviors and security motivation would be controlled by sensory and reafferent neural signals generated by performance of motor actions and by non-neural signals stimulated by the engagement in security behaviors. Using this framework, here we identify two exemplars of the likely neurochemical sources of satiety signals terminating security motivation.

a. Serotonin Receptors as a Component of the Negative Feedback. The review in sections II and III strongly indicates that serotonin plays a key role in OCD, although at the moment it remains unclear what this role is. Even so, a credible rationale can be assembled that serotonin, likely via 5 -HT2C receptors, forms a component in the multifaceted negative feedback yedasentience signal that shuts down security motivation, providing therapeutic relief in OCD.

Consistent with the therapeutic effects of SSRIs, Szechtman and Woody (2004) considered what neurobiological mechanisms underlie the yedasentience feedback and speculated that it was serotonin neurotransmission, based on the role of serotonin in terminating special motivations - sexual behavior (Lorrain et al., 1999) and eating (Blundell, 1991) — and based on brainstem serotonergic neurons having the requisite anatomic distribution for yedasentience pathways proposed in Fig. 2. Furthermore, the authors speculated that serotonin mediation of the yedasentience signal may be an instance of a more general rule that "central serotonergic neurons are involved in behavioral suppression (Soubrie, 1986) and inhibition of information flow (Spoont, 1992), effects that are often antagonistic to those of dopamine systems normally involved in facilitating active behavior (Antelman and Szechtman, 1975; Kapur and Remington, 1996; Robinson and Berridge, 1993; Wise and Bozarth, 1987)" (Szechtman and Woody, 2004, p. 121).

The satiety-like negative feedback effects may be produced from activation of 5-HT2C receptors. This had been shown clearly in studies on hunger motivation where 5-HT2C receptor agonists, such as Ro 60-0175, produce enhancement of satiety for food (Hewitt et al., 2002; Clifton, 2017) as measured in rodents using the method of "behavioral satiety sequence" (Rodgers et al., 2010). Importantly, opioid receptor antagonists and the combination of naltrexone with bupropion produce similar satiety-like effects, whereas atypical antipsychotic drugs such as olanzapine attenuate satiety from food (Clifton, 2017), in line with the inference that negative feedback is multifaceted and not a singular neurochemical entity.

As reviewed in section III.A, the administration of 5-HT2C receptor agonists has beneficial effects in some animal models of OCD. We suggest that this therapeutic benefit is produced, at least in part, through enhancement of satiety-like yedasentience feedback. Such an interpretation is evident most readily from findings with mCPP in the quinpirole-induced compulsive checking model of OCD (Tucci et al., 2013). Two results from that study are of particular relevance: 1) $\mathrm{mCPP}$ produced a dose-dependent reduction in the vigor of performance of checking behavior but no change in the focus with which it was performed; and 2) at the higher dose, mCPP increased the period of rest ("satiety") after a bout of checking in the quinpirole compulsive rat, albeit in concert with a reduction of vigor.

Are enhanced negative feedback and reduced vigor necessarily linked? Some coupling of vigor and satiety may be experimentally unavoidable because induction of "satiety" should diminish the incentive of motivational stimuli (Barrozo et al., 2010a,b) and thus motor vigor (Niv et al., 2007). However, the double effect with mCPP may result also from stimulating multiple serotonin receptors, given that $\mathrm{mCPP}$ is more aptly a nonselective 5-HT2A/2C agonist (Rajkumar et al., 2009; Rodriguez et al., 2017). The two receptor subtypes have a different distribution in the brain (Pompeiano et al., 1994) and sometimes opposite effects on behavior (Winstanley et al., 2004b; Boulougouris et al., 2008; Kontis et al., 2008; Halberstadt et al., 2009; Zeeb et al., 2010). Conceivably, satiety may be relatively more influenced by 5 -HT2C receptors, whereas motor vigor may be influenced by stimulation of 5-HT2A receptors. This hypothesis should be tested by evaluating, for example, the relative ranking of 5-HT2C and 5-HT2A agonists for their effects on compulsive checking, and in particular whether there is a greater influence of 
5-HT2C than 5-HT2A stimulation over postchecking satiety and vice versa over vigor.

A similar distinction between the effects of 5-HT2C and non-5-HT2C receptors on satiety and vigor may be apparent in an animal model of OCD using CPD 1, an agonist with high affinity and selectivity for 5-HT2C receptors (Rodriguez et al., 2017). Key study results were described earlier in section III.A.1; here we consider the relevance of the proposed distinction between effects on negative feedback and motor vigor.

The novel aspect of the study results of Rodriguez et al. (2017) is the dissociation produced by CPD 1 in two dependent variables that characterize scheduleinduced polydipsia, the test used to evaluate therapeutic usefulness of drugs for OCD. Unlike mCPP and other agonists that alter both dependent measures, CPD 1 had little effect on the number of motor responses (bar presses) directed at obtaining food but it substantially decreased the amount of drinking in between such motor behavior (Rodriguez et al., 2017). It makes sense to us as observers that the hungry animal lever presses to obtain food, and not for access to drink as water is freely available. The finding that CPD 1 (a highly selective agonist of 5-HT2C receptors) had little effect on the vigor of bar presses is consistent with the suggestion that motor vigor is controlled relatively less by 5 -HT2C receptors than some other receptors, perhaps 5-HT2A receptors. Given that 5-HT2C agonists produce enhancement of satiety for food (Hewitt et al., 2002; Clifton, 2017), the rat in the schedule-induced polydipsia paradigm may be less hungry after the administration of CPD 1. Indeed, lorcaserin (Thomsen et al., 2008), the 5-HT2C agonist approved for control of obesity, is said to produce a sense of satiety and absence of hunger (Bello and Liang, 2011). It is this CPD 1-induced greater satiety, we suggest, that diminishes the incentive value of water and so reduces the amount of drinking.

Studies in 5-HT2C receptor null mutant mice generally are consistent with 5 -HT2C receptors mediating a satiety-like negative feedback signal. These mice are hyperphagic and SMS activity is increased as evidenced by a stronger response to cues of potential danger (instigated by home cage cleaning) than to an actual threat from confinement in a restraining apparatus (Chou-Green et al., 2003b); their hyperactive behavior investigating novel objects is not widespread but spatially restricted (analogous to the spatially restricted routes of locomotion in quinpirole-treated rats; Eilam et al., 1989), giving the appearance of compulsive-like behavior (Chou-Green et al., 2003a), presumably because inhibitory signals mediated by 5 -HT2C receptors that would facilitate switching among different actions are diminished. Moreover, electrophysiological responses in the OFC and caudate nucleus are activated more easily (Rueter et al., 2000), suggesting diminished inhibitory influence in some brain regions in the absence of 5-HT2C receptors.
Although evidence implicates 5 -HT2C receptors in the yedasentience satiety-like negative feedback signal (proposed as deficient in OCD), the reviewed studies show that their involvement is not specific to SMS and OCD. As noted, a satiety-like effect is observed not only in security motivation but even more conspicuously in hunger motivation. Moreover, the inhibitory effects of 5-HT2C and/or some closely related 5-HT receptor subtypes extend more broadly to motor vigor. Hence, rather than being selective to SMS, the signal mediated by 5 -HT2C receptors carries negative feedback to a range of systems. In this respect, such serotonergic stimulation of 5-HT2C receptors is consistent with the operation of an infrastructure facility discussed in section V.B.1. As such, compounds like the highly selective 5-HT2C agonist, CD 1, should be of therapeutic value not only for OCD but also for a range of psychiatric and nonpsychiatric conditions.

At present, much basic research needs to be pursued to identify the neurochemical components underlying yedasentience and its neurobiological fault yielding OCD. Consistent with the supposition that the yedasentience negative feedback signal is most likely multifaceted, such research efforts should include investigation of the neurochemistry mediating proprioceptive and vestibular signaling and of motor control processing (Brown et al., 2011), because motor activity is proposed to generate the yedasentience feedback. Non-neural signals are also likely to contribute in generating yedasentience, and we turn next to this possibility.

b. Microglia "Off" Signals as a Component of the Negative Feedback. We generally think of the immune system as confronting the imminent threat from invasion by pathogens; however, it also partakes in more sedate tasks, including the maintenance and repair of brain tissue and the rewiring of brain circuits during learning (Kettenmann et al., 2013; Rodríguez-Iglesias et al., 2019). Microglia, a constituent of the immune system, are engaged in another function of relevance to the SMS. In fact, we propose that microglia are part of the security motivation network but whereas exteroceptors sense cues of potential danger in the external environment, microglia sense potential danger from within the internal environment of the brain and the body itself. Although such a conceptualization for the function of microglia has not been described in precisely these terms, it is implicit in discussions of the "housekeeping" surveillance and sensory functions of resting microglia (Nimmerjahn et al., 2005; Hanisch and Kettenmann, 2007; Ransohoff and Perry, 2009; Kettenmann et al., 2011; Tremblay et al., 2011; Benarroch, 2013; Hickman et al., 2013; Shemer et al., 2015; Deczkowska et al., 2018).

In their review of microglia as "neuroimmune-sensors of stress," Frank et al. (2019) note that perturbations that evoke the "fight/flight response" have characteristic 
effects both on behavior and on neurobiology, and that the latter includes effects on microglia that "secrete a number of immunological factors, which modulate the motivational state and cognitive function of the organism ... thereby providing a critical immunological input to neural circuits to help shape an organism's behavioral response to threats" (p. 176). The authors consider this arrangement of functional significance because "with threats may come harm and injury, thus hyper-vigilant microglia may be in a state of preparedness to respond rapidly to harm" (p. 182-183). Microglia and neurons share many of the same molecular signaling molecules (ion channels, neurohormone and neurotransmitter receptors), allowing each to respond directly to mediators of the stress response such as glucocorticoids and catecholamines, and rapidly coordinate an appropriate physiologic response to stress-induced harm (Frank et al., 2019).

Frank et al. (2019) consider the function of microglia in paradigms that involve relatively intense stress (the animal is experiencing electric shocks, is attacked and defeated in a fight by another animal, etc.) and these conditions are generally sufficient to induce a transition in microglia from a resting to an activated reactive phenotype. However, the situations of stress reviewed by the authors are in fact instances of confronting an actual, currently present danger, whereas what activates the SMS is instead the mere prospect of a threatpotential danger. In terms of mediators of the stress response such as glucocorticoids, potential danger cues that activate the SMS are also potent stimulators of the HPA axis. In fact, such a seemingly innocuous procedure as transferring the rat from its home cage to a new empty environment is a reliable procedure to stimulate a robust release of adrenal glucocorticoids (Brown and Martin, 1974; Szechtman et al., 1974; Hennessy and Levine, 1978), as indeed do most exposures to a novel situation that create uncertainty in the organism (Mason, 1968; Bassett et al., 1973; Pfister, 1979; Anisman, 2014). However, potential threat does not elicit the sympathetic "fight-or-flight" activation but instead, as part of the physiologic underpinnings of the SMS (see Fig. 3), attenuates inhibitory control over the parasympathetic component of the autonomic nervous system, priming the sympathetic system for quick activation in the case of danger materializing (Porges, 1995, 2007; Hinds et al., 2010; Woody and Szechtman, 2011). Unfortunately, subtler cues such as for potential danger have not yet been studied in research on microglia. However, we suggest that potential danger does have an influence on microglia but, unlike present danger, produces an alerted surveillance state in the microglia without producing a transformation in the microglia to the classically recognized activated reactive phenotypes. To highlight this role of microglia as a component of SMS, Fig. 4 provides a schema of the immunologic component associated with security motivation; this schema complements the depiction in Fig. 3 of the physiologic network associated with security motivation.

What would be the added adaptive value in the SMS regulating microglia? Could the same job be accomplished without SMS control? Precautionary behaviors like grooming and washing are effective only if the pathogen load is small, because this small load can be dealt with effectively through such actions. In contrast, such behavior becomes overwhelmed and is ineffective when the pathogen load grows to be high. Hence, the adaptive value of washing and grooming precautionary behaviors lies in limiting the opportunities for pathogens to multiply rapidly and reach high numbers. Indeed, if the numbers are high and an infection is already present, other, nonprecautionary behaviors are called for, such as sickness behavior as well as a fullblown immune response to manage the high load of pathogens inside the organism. Therefore, it makes sense that if the SMS is stimulated by exteroceptive cues of potential contagion or infection, then in addition to the evoked precautionary responses of washing and grooming there is also activation of an associated internal response to probe for pathogens that may have managed to enter the inside of the organism. We postulate that the Appraisal and Security Motivation modules (Fig. 1) communicate the exteroceptive signal of potential infection to microglia in contact with SMS neural sites, and that microglia in turn send out signaling molecules that prime a set of resting microglia into an "alerted" state to probe the internal environment for the presence of pathogens. Should the alerted microglia come in contact with the expected pathogens, this would trigger an appropriate immune response, dependent on the rate of influx of such pathogens. If the rate is low, then a "mopping up" operation is activated to remove the few intruders; if the rate is high, then a transformation of the microglia into an activated reactive phenotype is required to negate the effects of the invading pathogens.

What happens to the alerted state when the probing microglia do not encounter any expected pathogens? Do they continue operating in the alerted phenotype or revert back to the resting state? Because of "the potentially damaging consequences of an immune reaction ... microglial activation is thus a highly regulated process" (Kettenmann et al., 2011, p. 468). Hence, most models suggest that in the healthy state, once the job of the stimulated microglia is done there are negative feedback signals that deactivate (or "calm") those microglia and return them to the baseline resting state (Hanisch and Kettenmann, 2007; Kettenmann et al., 2011; Benarroch, 2013). Accordingly, in the case of an activated SMS and small pathogen load, precautionary behavior, through triggering signaling molecules that communicate between the neural and microglial components of the SMS, would exert a negative feedback 


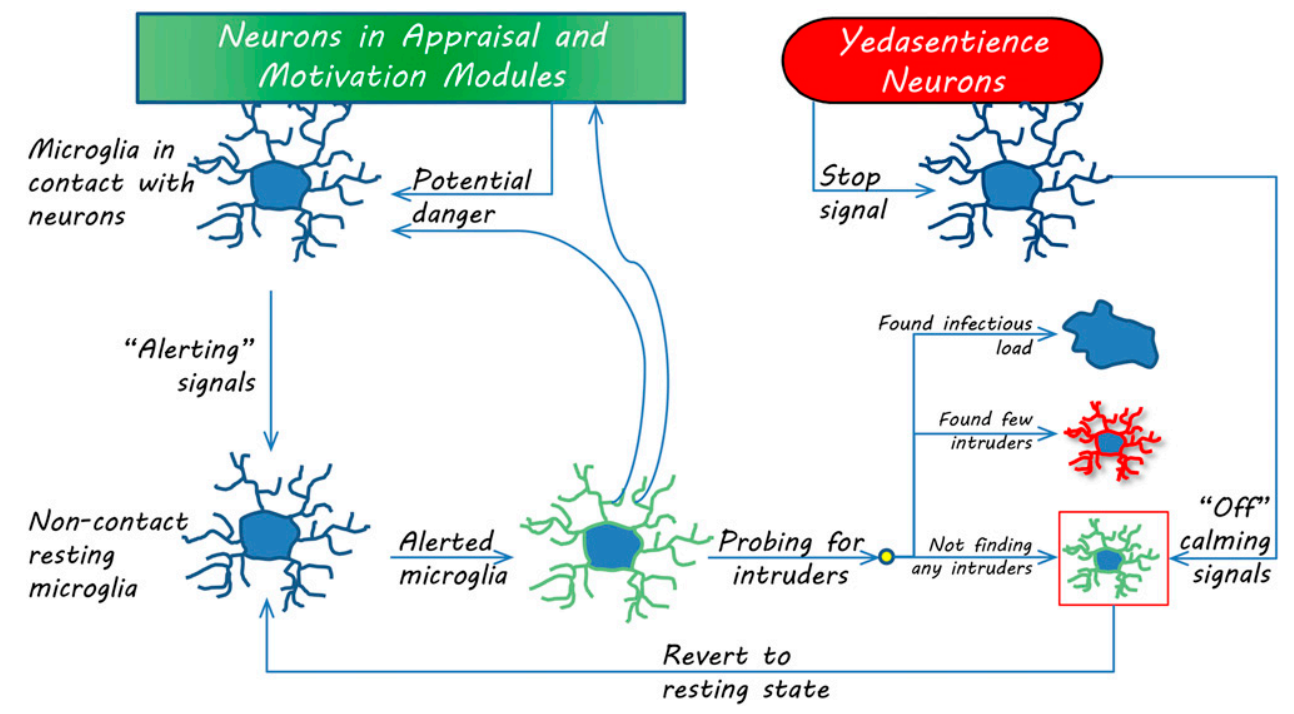

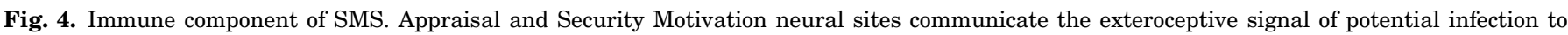

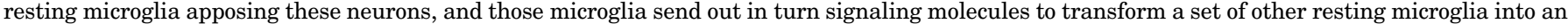

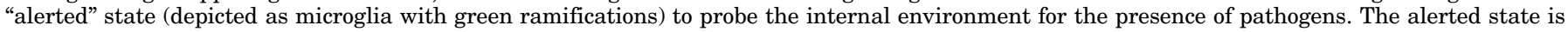

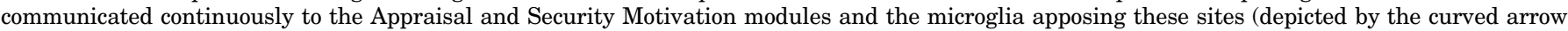

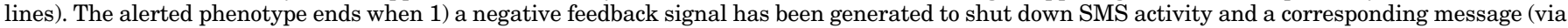

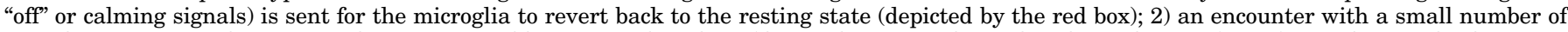

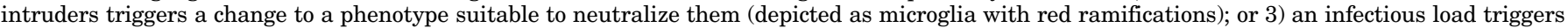
the transformation into reactive amoeboid phenotype (depicted by blue cells without ramifications).

effect on the microglia, returning them to a resting state (see Fig. 4).

In OCD, if the operation of the SMS fails to return the microglia to a resting state, they would continue to be vigilant and likely contribute to keeping security motivation activated, in the context of a bidirectional interaction of the microglial with the neural and HPA components of the SMS.

From the perspective of how to terminate SMS activity and arrest continued restimulation by alerted microglia, good leads are available: There is a substantial amount of knowledge about the molecular mechanisms that deactivate (or "calm") microglia, with many inhibitory "off" signals exerting their effects on immune cell "checkpoints" (Deczkowska et al., 2018; Graeber, 2018; Wei et al., 2018). Neurons have a variety of ways to signal a healthy state and thus provide a calming effect on the microglia. According to Benarroch (2013), these include "contact receptor-ligand interactions mediated by neuronal CD200 and microglial CD200 receptor; secreted molecules such as fractalkine (CX3CL1) that is bound to the neuronal membrane and then undergoes cleavage to activate microglial CX3CR1 receptors; and triggering receptor in myeloid cells-2 (TREM2)" (p. 108). Benarroch (2013) also considers that endocannabinoids and possibly other lipid mediators released by microglia act to suppress proinflammatory responses, thus providing another avenue of negative feedback. A more exhaustive identification of possible microglia "off" signaling molecules is provided in Biber et al. (2007), Kettenmann et al. (2011), and Benarroch (2013).
Some of these "off" signals may constitute a facet of SMS and yedasentience, and hence may be worth exploring as potential leads in the psychopharmacology of OCD.

\section{E. Roadmap}

In this section, we have distinguished three different ways to approach psychopharmacological research for the treatment of OCD: enhancing infrastructure, facilitating relearning, and fixing a weak link. Figure 5 shows where these three major possibilities would exert their impact on the operation of the SMS. In particular, drugs to enhance infrastructure would boost the functioning of the SMS as a whole, thereby helping to shift the network toward better overall function. Drugs to facilitate relearning, together with appropriate learning opportunities such as those provided by psychotherapy, would help to recalibrate system modules in the SMS (namely, the Appraisal of Potential Danger and the Security-Related Programs modules). Finally, drugs to fix a weak link would help to compensate for a specific deficit in the crucial negative-feedback loop of the SMS (with the possible sites of the deficit depicted by the red Xs in Fig. 5).

Table 4 elaborates on Fig. 5 by providing specific examples for each of these approaches and considering the relevant neurobiological targets, the therapeutic objectives, and the hypothesized mechanisms for a therapeutic effect in OCD. The table also notes the corresponding sections of the text that provide more details about leads for possible agents and targets to investigate. Together, Fig. 5 and Table 4 outline our proposed 


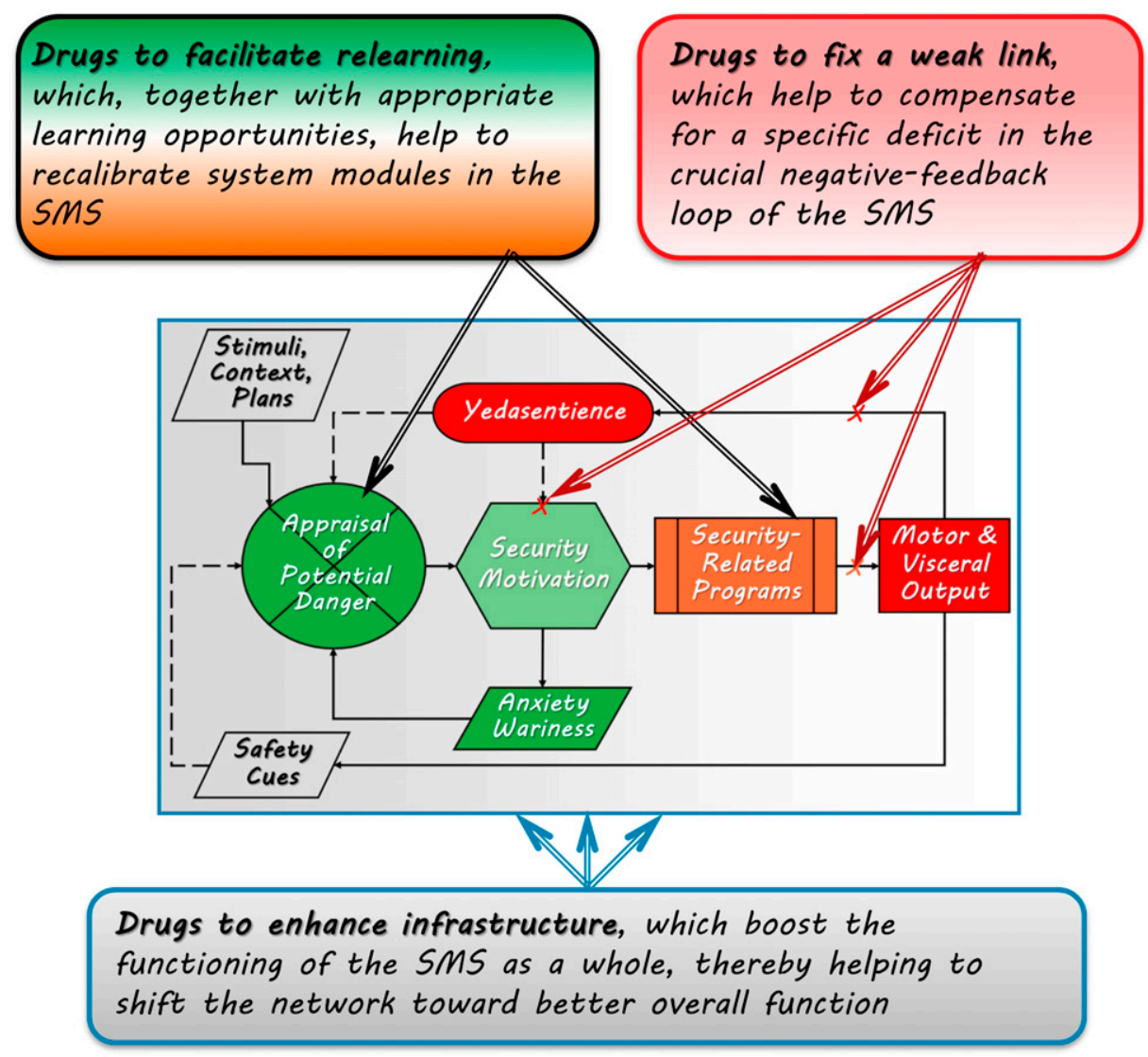

Fig. 5. Pharmacological strategies to rectify SMS function in the treatment of OCD. There are three different ways to approach psychopharmacological research relating to the treatment of OCD: enhancing infrastructure, facilitating relearning, and fixing a weak link. First, drugs to enhance infrastructure boost the functioning of the SMS as a whole, shifting it toward better overall function. Second, drugs to facilitate relearning, together with appropriate learning opportunities, recalibrate the Appraisal of Potential Danger and the Security-Related Programs modules of the SMS. Third, drugs to fix a weak link compensate for a specific deficit in the negative-feedback loop of the SMS; possible sites of this deficit are indicated by red Xs. Not highlighted in the figure is the range of nonpharmacological means discussed in the text that could also be employed either alone or as augmentation in a strategy to rectify SMS function. For instance, rTMS and tDCS may be useful to enhance neural plasticity in a treatment approach that seeks to facilitate relearning or fix a weak link; exercise and a consistent schedule of daily activity may entrain circadian rhythmicity and enhance infrastructure activity.

"preclinical roadmap" for future psychopharmacological research on OCD.

\section{Summary and Conclusions}

To conclude, we briefly recap the main arguments put forth in this review. With regard to clinical practice both in pharmacotherapy and also in psychotherapy for OCD, evidence of successful treatment has led researchers to propose various presumed etiologies of the disorder. Unfortunately, these presumptions have been put forth despite the lack of direct evidence about what the underlying mechanisms for treatment success may actually be. Moreover, the inference of etiology from treatment success involves, as we have seen, a logical fallacy ("the fallacy of confirming the consequence") and, in any case, has been criticized for yielding "few insights or novel treatments" (Goodman et al., 2014).

Turning to basic research concerning the neurobiology of OCD, we have found a myriad of diverse and often contradictory findings involving multiple levels and brain systems, which are resistant to the attempt to draw them into a coherent overall picture. It seems clear that a unifying theoretical perspective is unlikely to emerge spontaneously from these many assorted findings, and some critics have recently argued that the fault lies in the predominantly atheoretical approach to psychopathology research, as typified by DSM diagnosis as its linchpin.

Given this state of affairs, we have proposed a new perspective for preclinical research on OCD, which strongly links OCD with a biologically ancient brain system that evolved to manage risk from the prospect of relatively unlikely but potentially survival-threatening events, such as a contagion by disease or an attack by predators. This special motivation system-the SMSis specifically directed toward the detection of potential danger, and its output is precautionary behaviors that would ameliorate such dangers were they to eventuate. The normal functioning of the SMS and the consideration of its component processes both link OCD with the spectrum of normal, adaptive responses to cues for potential danger, and they also provide the framework for explaining how OCD may be the result of 
TABLE 4

Roadmap for potential psychopharmacological interventions in OCD

\begin{tabular}{|c|c|c|c|c|}
\hline $\begin{array}{l}\text { Strategy of } \\
\text { Intervention }\end{array}$ & $\begin{array}{c}\text { Example of Neurobiological } \\
\text { Target }\end{array}$ & Therapeutic Objective & $\begin{array}{l}\text { Hypothesized Mechanism for Therapeutic } \\
\text { Effect in OCD }\end{array}$ & Section \\
\hline \multicolumn{5}{|l|}{$\begin{array}{l}\text { Enhance } \\
\text { infrastructure }\end{array}$} \\
\hline & $\begin{array}{l}\text { Monoamine } \\
\text { neurotransmitter systems }\end{array}$ & $\begin{array}{l}\text { To optimize the balance in } 5-\mathrm{HT} \text {, } \\
\text { dopamine, and norepinephrine } \\
\text { neurotransmission }\end{array}$ & $\begin{array}{l}\text { Facilitation of SMS shifts from arousal to } \\
\text { satiety }\end{array}$ & V.B.1 \\
\hline & Circadian rhythmicity & $\begin{array}{l}\text { To maximize entrainment and synchrony } \\
\text { of oscillatory processes }\end{array}$ & $\begin{array}{l}\text { Reliance of SMS on well coordinated } \\
\text { circadian processes }\end{array}$ & V.B.2 \\
\hline & NAD metabolism & $\begin{array}{l}\text { To optimize a wide range of cellular } \\
\text { functions }\end{array}$ & $\begin{array}{l}\text { Reliance of SMS on efficient cellular } \\
\text { functioning }\end{array}$ & V.B.3 \\
\hline \multicolumn{5}{|l|}{$\begin{array}{l}\text { Facilitate } \\
\quad \text { relearning }\end{array}$} \\
\hline & $\begin{array}{l}\text { SMS module for Appraisal of } \\
\text { Potential Danger }\end{array}$ & $\begin{array}{l}\text { To facilitate relearning of appraisal of } \\
\text { potential danger }\end{array}$ & $\begin{array}{l}\text { Augmentation of neural plasticity to aid } \\
\text { recalibration of SMS }\end{array}$ & V.C \\
\hline & SMS output modules & $\begin{array}{l}\text { To facilitate relearning of behavioral } \\
\text { response repertoire }\end{array}$ & $\begin{array}{l}\text { Augmentation of neural plasticity and } \\
\text { provision of enhanced affective cues for } \\
\text { SMS shut down }\end{array}$ & V.C \\
\hline \multicolumn{5}{|l|}{ Fix a weak link } \\
\hline & $\begin{array}{l}5 \text {-HT signaling via } 5 \text {-HT2C } \\
\text { receptors }\end{array}$ & $\begin{array}{l}\text { To restore negative feedback } \\
\text { yedasentience signaling that shuts } \\
\text { down SMS }\end{array}$ & Increase of serotonergic-mediated satiety & V.D.2.a \\
\hline & $\begin{array}{l}\text { SMS-neuroimmune } \\
\text { communication }\end{array}$ & $\begin{array}{l}\text { To use microglia "off" signaling molecules } \\
\text { to shut down SMS }\end{array}$ & $\begin{array}{l}\text { SMS negative feedback pathways employ } \\
\text { same transmitters as microglia "off" } \\
\text { signals }\end{array}$ & V.D.2.b \\
\hline
\end{tabular}

dysfunctional dynamics in this biologically primitive system. Specifically, OCD may result from dysfunction in the capacity to terminate the activation of this system through the normal route of security-related behavior. For those with OCD, cues for potential danger activate the SMS the same as they do for all people; however, once their SMS is activated, their security-related thoughts and actions persist abnormally because such behavior is abnormally ineffective in providing negative feedback to shut the system down. Moreover, we can specify the basic functional components of the SMS and use these, together with knowledge from basic neuroscience, to organize generative hypotheses about the brain circuits and neurophysiology that are implicated-including, as shown in Figs. 2-4, feedback loops involving cortico-thalamico-cortical circuits, the HPA axis, and the microglia, respectively. OCD represents a particular maladaptive variant of how this system functions-its symptoms are not a strange collection of puzzling fragments, but expressions, in exaggerated form, of a normal motivation system.

Making use of this theoretical framework, we have proposed a new perspective on psychopharmacological research on OCD, in which we have distinguished three major approaches. The first is what we have called the elixir of life approach, in which rather than attempting to have a direct effect on an underlying neurobiological fault in OCD, the aim of psychopharmacology is a general enhancement of the infrastructure facilities that support brain functioning globally and hence may improve the functioning of a faulty SMS. The idea that a range of factors may have a broad, comparatively nonspecific but ameliorative effect on SMS functioning in OCD is supported by the diverse findings in the neurobiological literature reviewed in section III.
The second major approach we have discussed is pharmacotherapy to facilitate relearning, such as occurs in psychotherapy for OCD. Drugs such as cognitive enhancers might accelerate the learning process in psychologic treatment that shifts patients' SMS functioning toward more adaptive ways of managing potential danger. Because a common stereotype about psychotherapy is that to be truly effective it must be a long and slow process, the goal of accelerating the learning process might seem odd at first. Nonetheless, the available data suggest that rapid progress is associated with better long-term outcomes, and therapeutic intervention of even very short duration (e.g., 4 days) can be lastingly effective (Hansen et al., 2018).

The third major approach we have discussed is psychopharmacology aimed at targeting specific pathways in the SMS network, thus "fixing a weak link." Given the focus in the SMS theory of OCD on stopping dynamics, research might profitably focus on drugs that could enhance the negative feedback function, associated with the satiety signal of yedasentience, crucial for shutting down the SMS. However, the necessary foundation for such work will likely be further basic research revealing the underlying nature and normal functioning of the SMS, prerequisite to better understanding the neurobiology of the specific fault in OCD that requires intervention. Nonetheless, we believe we have been able to sketch some intriguing hypotheses about the serotonergic neurochemistry for satiety of security motivation as well as how microglia and the HPA axis may provide "off" signals for the SMS.

Finally, it is important to point out that the three major possibilities we have discussed are not mutually exclusive. Our basic theme is to look at psychopathology as the dysfunctional expression of a normal, special 
motivation system. This system may have a specific neurobiological fault in OCD, is open to recalibration with new learning under the right circumstances, and is crucially supported by infrastructure facilities that may need to be boosted for better functioning.

\section{Acknowledgments}

We thank Dr. De Wet Wolmarans for scholarly contributions during the writing of this review article.

\section{Authorship Contributions}

Wrote or contributed to the writing of the manuscript: Szechtman, Harvey, Woody, Hoffman.

\section{References}

Abdolhosseinzadeh S, Alizadeh N, Shams J, Asadi S, and Ahmadiani A (2019) BDNF association study with obsessive-compulsive disorder, its clinical characteristics, and response to fluvoxamine-treatment in Iranian patients. Exp Clin Psychopharmacol DOI: 10.1037/pha0000297 [published ahead of print].

Abed RT and de Pauw KW (1998) An evolutionary hypothesis for obsessive compulsive disorder: a psychological immune system? Behav Neurol 11:245-250.

Abramowitz JS (2018) Presidential address: are the obsessive-compulsive related disorders related to obsessive-compulsive disorder? A critical look at DSM-5's new category. Behav Ther 49:1-11.

Abramowitz JS, Foa EB, and Franklin ME (2003) Exposure and ritual prevention for obsessive-compulsive disorder: effects of intensive versus twice-weekly sessions. $J$ Consult Clin Psychol 71:394-398.

Abramowitz JS, Nelson CA, Rygwall R, and Khandker M (2007) The cognitive mediation of obsessive-compulsive symptoms: a longitudinal study. J Anxiety Disord 21 91-104.

Abrantes AM, Brown RA, Strong DR, McLaughlin N, Garnaat SL, Mancebo M, Riebe D, Desaulniers J, Yip AG, Rasmussen S, et al. (2017) A pilot randomized controlled trial of aerobic exercise as an adjunct to OCD treatment. Gen Hosp Psychiatry 49:51-55.

Abrantes AM, Farris SG, Brown RA, Greenberg BD, Strong DR, McLaughlin NC, and Riebe D (2019) Acute effects of aerobic exercise on negative affect and obsessions and compulsions in individuals with obsessive-compulsive disorder. J Affect Disord 245:991-997.

Adams DB (1979) Brain mechanisms for offense, defense, and submission. Behav Brain Sci 2:201-213.

Adler CM, McDonough-Ryan P, Sax KW, Holland SK, Arndt S, and Strakowski SM (2000) fMRI of neuronal activation with symptom provocation in unmedicated patients with obsessive compulsive disorder. J Psychiatr Res 34:317-324.

Afshar H, Roohafza H, Mohammad-Beigi H, Haghighi M, Jahangard L, Shokouh P, Sadeghi M, and Hafezian H (2012) N-acetylcysteine add-on treatment in refractory obsessive-compulsive disorder: a randomized, double-blind, placebo-controlled trial. J Clin Psychopharmacol 32:797-803.

Ahmari SE, Spellman T, Douglass NL, Kheirbek MA, Simpson HB, Deisseroth K, Gordon JA, and Hen R (2013) Repeated cortico-striatal stimulation generates persistent OCD-like behavior. Science 340:1234-1239.

Alboni S, van Dijk RM, Poggini S, Milior G, Perrotta M, Drenth T, Brunello N, Wolfer DP, Limatola C, Amrein I, et al. (2017) Fluoxetine effects on molecular, cellular and behavioral endophenotypes of depression are driven by the living environment. Mol Psychiatry 22:552-561.

Alex KD and Pehek EA (2007) Pharmacologic mechanisms of serotonergic regulation of dopamine neurotransmission. Pharmacol Ther 113:296-320.

Alexander GE, DeLong MR, and Strick PL (1986) Parallel organization of functionally segregated circuits linking basal ganglia and cortex. Annu Rev Neurosci 9:357-381.

Alkhatib AH, Dvorkin-Gheva A, and Szechtman H (2013) Quinpirole and 8-OH-DPAT induce compulsive checking behavior in male rats by acting on different functional parts of an OCD neurocircuit. Behav Pharmacol 24:65-73.

Allen AP, Hutch W, Borre YE, Kennedy PJ, Temko A, Boylan G, Murphy E, Cryan JF, Dinan TG, and Clarke G (2016) Bifidobacterium longum 1714 as a translational psychobiotic: modulation of stress, electrophysiology and neurocognition in healthy volunteers. Transl Psychiatry 6:e939.

Alpak G and Karamustafalioglu O (2009) Female reproductive cycle events in symptom worsening and onset of obsessive-compulsive disorder. Eur Neuropsychopharmacol 19:S592.

Altemus M (2006) Sex differences in depression and anxiety disorders: potential biological determinants. Horm Behav 50:534-538.

Altemus M, Jacobson KR, Debellis M, Kling M, Pigott T, Murphy DL, and Gold PW (1999) Normal CSF oxytocin and NPY levels in OCD. Biol Psychiatry 45:931-933.

Altemus M, Pigott T, Kalogeras KT, Demitrack M, Dubbert B, Murphy DL, and Gold PW (1992) Abnormalities in the regulation of vasopressin and corticotropin releasing factor secretion in obsessive-compulsive disorder. Arch Gen Psychiatry 49:9-20.

American Psychiatric Association (2013) Diagnostic and Statistical Manual of Mental Disorders (DSM-5®), American Psychiatric Association, Arlington, VA.

Ananth J (1986) Clomipramine: an antiobsessive drug. Can J Psychiatry 31:253-258.

Anderson ML (2016) Neural reuse in the organization and development of the brain. Dev Med Child Neurol 58 (Suppl 4):3-6.

Anderson ML and Finlay BL (2014) Allocating structure to function: the strong links between neuroplasticity and natural selection. Front Hum Neurosci 7:918.

Andersson E, Hedman E, Enander J, Radu Djurfeldt D, Ljótsson B, Cervenka S, Isung J, Svanborg C, Mataix-Cols D, Kaldo V, et al. (2015) D-cycloserine vs placebo as adjunct to cognitive behavioral therapy for obsessive-compulsive disorder and interaction with antidepressants: a randomized clinical trial. JAMA Psychiatry $\mathbf{7 2}$ $659-667$.

Anisman H (2014) An Introduction to Stress \& Health, Sage, London.

Antelman SM and Chiodo LA (1981) Repeated antidepressant treatments induce a long-lasting dopamine autoreceptor subsensitivity: is daily treatment necessary for clinical efficacy? [proceedings]. Psychopharmacol Bull 17:92-94.

Antelman SM, Levine J, and Gershon S (2000) Time-dependent sensitization: the odyssey of a scientific heresy from the laboratory to the door of the clinic. Mol Psychiatry 5:350-356.

Antelman SM and Szechtman H (1975) Tail pinch induces eating in sated rats which appears to depend on nigrostriatal dopamine. Science 189:731-733.

Aouizerate B, Guehl D, Cuny E, Rougier A, Bioulac B, Tignol J, and Burbaud P (2004) Pathophysiology of obsessive-compulsive disorder: a necessary link between phenomenology, neuropsychology, imagery and physiology. Prog Neurobiol 72:195-221.

Aoyama K and Nakaki T (2013) Impaired glutathione synthesis in neurodegeneration. Int J Mol Sci 14:21021-21044.

Aoyama K, Suh SW, Hamby AM, Liu J, Chan WY, Chen Y, and Swanson RA (2006) Neuronal glutathione deficiency and age-dependent neurodegeneration in the EAAC1 deficient mouse. Nat Neurosci 9:119-126.

Arendt J (2018) Approaches to the pharmacological management of jet lag. Drugs 78 $1419-1431$

Arnold PD, Askland KD, Barlassina C, Bellodi L, Bienvenu O, Black D, Bloch M, Brentani H, Burton CL, and Camarena B; International Obsessive Compulsive Disorder Foundation Genetics Collaborative (IOCDF-GC) and OCD Collaborative Genetics Association Studies (OCGAS) (2018) Revealing the complex genetic architecture of obsessive-compulsive disorder using meta-analysis. Mol Psychiatry 23: $1181-1188$

Arnold PD, Macmaster FP, Richter MA, Hanna GL, Sicard T, Burroughs E, Mirza Y, Easter PC, Rose M, Kennedy JL, et al. (2009) Glutamate receptor gene (GRIN2B) associated with reduced anterior cingulate glutamatergic concentration in pediatric obsessive-compulsive disorder. Psychiatry Res 172:136-139.

Arnold PD, Sicard T, Burroughs E, Richter MA, and Kennedy JL (2006) Glutamate transporter gene SLC1A1 associated with obsessive-compulsive disorder. Arch Gen Psychiatry 63:769-776.

Aron AR (2009) Introducing a special issue on stopping action and cognition. Neurosci Biobehav Rev 33:611-612.

Atmaca M, Onalan E, Yildirim H, Yuce H, Koc M, Korkmaz S, and Mermi O (2011) Serotonin transporter gene polymorphism implicates reduced orbito-frontal cortex in obsessive-compulsive disorder. J Anxiety Disord 25:680-685.

Atmaca M, Tezcan E, Kuloglu M, and Ustundag B (2005) Plasma nitrate values in patients with obsessive-compulsive disorder. Psychiatry Clin Neurosci 59:621-623.

Australian Psychological Society (APS) (2010) Evidence-Based Psychological Interventions in the Treatment of Mental Disorders: A Literature Review, 3rd ed, Australian Psychological Society, Melbourne, Australia.

Azmitia EC (2001) Modern views on an ancient chemical: serotonin effects on cell proliferation, maturation, and apoptosis. Brain Res Bull 56:413-424.

Baer L, Rauch SL, Ballantine HT Jr, Martuza R, Cosgrove R, Cassem E, Giriunas I, Manzo PA, Dimino C, and Jenike MA (1995) Cingulotomy for intractable obsessivecompulsive disorder. Prospective long-term follow-up of 18 patients. Arch Gen Psychiatry 52:384-392.

Bagdy G, Graf M, Anheuer ZE, Modos EA, and Kantor S (2001) Anxiety-like effects induced by acute fluoxetine, sertraline or m-CPP treatment are reversed by pretreatment with the 5-HT2C receptor antagonist SB-242084 but not the 5-HT1A receptor antagonist WAY-100635. Int J Neuropsychopharmacol 4:399-408.

Bailly D, Servant D, Dewailly D, Beuscart R, Racadot A, Fossati P, and Parquet PJ (1994) Corticotropin releasing factor stimulation test in obsessive compulsive disorder. Biol Psychiatry 35:143-146.

Ballester González J, Dvorkin-Gheva A, Silva C, Foster JA, and Szechtman H (2015) Nucleus accumbens core and pathogenesis of compulsive checking. Behav Pharmacol 26:200-216.

Balsa JA, Sánchez-Franco F, Pazos F, Lara JI, Lorenzo MJ, Maldonado G, and Cacicedo L (1998) Direct action of serotonin on prolactin, growth hormone, corticotropin and luteinizing hormone release in cocultures of anterior and posterior pituitary lobes: autocrine and/or paracrine action of vasoactive intestinal peptide. Neuroendocrinology 68:326-333.

Bandelow B, Baldwin D, Abelli M, Altamura C, Dell'Osso B, Domschke K, Fineberg NA, Grünblatt E, Jarema M, Maron E, et al. (2016) Biological markers for anxiety disorders, OCD and PTSD - a consensus statement. Part I: neuroimaging and genetics. World J Biol Psychiatry 17:321-365.

Bandelow B, Baldwin D, Abelli M, Bolea-Alamanac B, Bourin M, Chamberlain SR, Cinosi E, Davies S, Domschke K, Fineberg N, et al. (2017) Biological markers for anxiety disorders, OCD and PTSD: a consensus statement. Part II: neurochemistry, neurophysiology and neurocognition. World J Biol Psychiatry 18: $162-214$

Bari AA, Mikell CB, Abosch A, Ben-Haim S, Buchanan RJ, Burton AW, Carcieri S, Cosgrove GR, D'Haese PF, Daskalakis ZJ, et al. (2018) Charting the road forward in psychiatric neurosurgery: proceedings of the 2016 American Society for Stereotactic and Functional Neurosurgery workshop on neuromodulation for psychiatric disorders. J Neurol Neurosurg Psychiatry 89:886-896.

Barnes DS (2000) Historical perspectives on the etiology of tuberculosis. Microbes Infect 2:431-440.

Barrozo RB, Gadenne C, and Anton S (2010a) Switching attraction to inhibition: mating-induced reversed role of sex pheromone in an insect. $J$ Exp Biol 213: 2933-2939.

Barrozo RB, Jarriault D, Simeone X, Gaertner C, Gadenne C, and Anton S (2010b) Mating-induced transient inhibition of responses to sex pheromone in a male moth is not mediated by octopamine or serotonin. J Exp Biol 213:1100-1106.

Bassett JR, Cairncross KD, and King MG (1973) Parameters of novelty, shock predictability and response contingency in corticosterone release in the rat. Physiol Behav 10:901-907. 
Baxter LR (1990) Brain imaging as a tool in establishing a theory of brain pathology in obsessive compulsive disorder. J Clin Psychiatry 51 (Suppl):22-25, discussion 26.

Baxter LR Jr (1992) Neuroimaging studies of obsessive compulsive disorder. Psychiatr Clin North Am 15:871-884.

Baxter LR Jr, Schwartz JM, Bergman KS, Szuba MP, Guze BH, Mazziotta JC, Alazraki A, Selin CE, Ferng HK, Munford P, et al. (1992) Caudate glucose metabolic rate changes with both drug and behavior therapy for obsessive-compulsive disorder. Arch Gen Psychiatry 49:681-689.

Bear RE, Fitzgerald P, Rosenfeld JV, and Bittar RG (2010) Neurosurgery for obsessive-compulsive disorder: contemporary approaches. J Clin Neurosci 17:1-5.

Beardsley PM and Hauser KF (2014) Glial modulators as potential treatments of psychostimulant abuse. Adv Pharmacol 69:1-69.

Belenky P, Bogan KL, and Brenner C (2007) $\mathrm{NAD}^{+}$metabolism in health and disease. Trends Biochem Sci 32:12-19.

Bello NT and Liang NC (2011) The use of serotonergic drugs to treat obesity--is there any hope? Drug Des Devel Ther 5:95-109.

Belloch A, Cabedo E, and Carrio C (2008) Empirically grounded clinical interventions: cognitive versus behaviour therapy in the individual treatment of obsessivecompulsive disorder: changes in cognitions and clinically significant outcomes at post-treatment and one-year follow-up. Behav Cogn Psychother 36:521-540.

Benabid AL (2003) Deep brain stimulation for Parkinson's disease. Curr Opin Neurobiol 13:696-706.

Benabid AL, Benazzouz A, Hoffmann D, Limousin P, Krack P, and Pollak P (1998) Long-term electrical inhibition of deep brain targets in movement disorders. $M o v$ Disord 13 (Suppl 3):119-125.

Benarroch EE (2013) Microglia: multiple roles in surveillance, circuit shaping, and response to injury. Neurology 81:1079-1088.

Benito E and Barco A (2010) CREB's control of intrinsic and synaptic plasticity: implications for CREB-dependent memory models. Trends Neurosci 33:230-240.

Benkelfat C, Nordahl TE, Semple WE, King AC, Murphy DL, and Cohen RM (1990) Local cerebral glucose metabolic rates in obsessive-compulsive disorder. Patients treated with clomipramine. Arch Gen Psychiatry 47:840-848.

Bennett MR (2010) Synapse regression in depression: the role of 5-HT receptors in modulating NMDA receptor function and synaptic plasticity. Aust N Z J Psychiatry 44:301-308.

Ben-Pazi A, Szechtman H, and Eilam D (2001) The morphogenesis of motor rituals in rats treated chronically with the dopamine agonist quinpirole. Behav Neurosci $\mathbf{1 1 5}$ : 1301-1317.

Berk M, Malhi GS, Gray LJ, and Dean OM (2013) The promise of N-acetylcysteine in neuropsychiatry. Trends Pharmacol Sci 34:167-177.

Berlim MT, Neufeld NH, and Van den Eynde F (2013) Repetitive transcranial magnetic stimulation (rTMS) for obsessive-compulsive disorder (OCD): an exploratory meta-analysis of randomized and sham-controlled trials. J Psychiatr Res 47: 999-1006.

Bernard S, Gonze D, Cajavec B, Herzel H, and Kramer A (2007) Synchronizationinduced rhythmicity of circadian oscillators in the suprachiasmatic nucleus. PLoS Comput Biol 3:e68.

Berridge KC, Aldridge JW, Houchard KR, and Zhuang X (2005) Sequential superstereotypy of an instinctive fixed action pattern in hyper-dopaminergic mutant mice: a model of obsessive compulsive disorder and Tourette's. BMC Biol 3:4.

Berrios GE (1997) The origins of psychosurgery: Shaw, Burckhardt and Moniz. Hist Psychiatry 8:61-81.

Berrios GE and Marková IS (2002) The concept of neuropsychiatry: a historical overview. J Psychosom Res 53:629-638.

Beste C, Willemssen R, Saft C, and Falkenstein M (2010) Response inhibition subprocesses and dopaminergic pathways: basal ganglia disease effects. Neuropsychologia 48:366-373.

Bhaskara S (2019) N-Acetylcysteine augmentation in refractory obsessive-compulsive disorder. J Psychiatry Neurosci 44:215-216.

Bianchi MG, Bardelli D, Chiu M, and Bussolati O (2014) Changes in the expression of the glutamate transporter EAAT3/EAAC1 in health and disease. Cell Mol Life Sci 71:2001-2015.

Biber K, Neumann H, Inoue K, and Boddeke HW (2007) Neuronal 'on' and 'off signals control microglia. Trends Neurosci 30:596-602.

Bienvenu OJ, Wang Y, Shugart YY, Welch JM, Grados MA, Fyer AJ, Rauch SL, McCracken JT, Rasmussen SA, Murphy DL, et al. (2009) Sapap3 and pathological grooming in humans: results from the OCD collaborative genetics study. Am J Med Genet B Neuropsychiatr Genet 150B:710-720.

Bigos KL, Folan MM, Jones MR, Haas GL, Kroboth FJ, and Kroboth PD (2009) Dysregulation of neurosteroids in obsessive compulsive disorder. J Psychiatr Res 43: $442-445$.

Blanchard DC, Griebel G, Pobbe R, and Blanchard RJ (2011) Risk assessment as an evolved threat detection and analysis process. Neurosci Biobehav Rev 35:991-998.

Bloch MH, Landeros-Weisenberger A, Kelmendi B, Coric V, Bracken MB, and Leckman JF (2006) A systematic review: antipsychotic augmentation with treatment refractory obsessive-compulsive disorder. Mol Psychiatry 11:622-632.

Blundell J (1991) Pharmacological approaches to appetite suppression. Trends Pharmacol Sci 12:147-157.

Bode L, Zimmermann W, Ferszt R, Steinbach F, and Ludwig H (1995) Borna disease virus genome transcribed and expressed in psychiatric patients. Nat Med 1:232-236.

Boedhoe PS, Schmaal L, Abe Y, Ameis SH, Arnold PD, Batistuzzo MC, Benedetti F, Beucke JC, Bollettini I, Bose A, et al.; ENIGMA OCD Working Group (2017) Distinct subcortical volume alterations in pediatric and adult OCD: a worldwide meta- and mega-analysis. Am J Psychiatry 174:60-69.

Boedhoe PSW, Schmaal L, Abe Y, Alonso P, Ameis SH, Anticevic A, Arnold PD, Batistuzzo MC, Benedetti F, Beucke JC, et al.; ENIGMA OCD Working Group (2018) Cortical abnormalities associated with pediatric and adult obsessivecompulsive disorder: findings from the ENIGMA Obsessive-Compulsive Disorder Working Group. Am J Psychiatry 175:453-462.
Boes AD, Prasad S, Liu H, Liu Q, Pascual-Leone A, Caviness VS Jr, and Fox MD (2015) Network localization of neurological symptoms from focal brain lesions. Brain 138:3061-3075.

Boix J, Llansola M, Cabrera-Pastor A, and Felipo V (2011) Metabotropic glutamate receptor 5 modulates the nitric oxide-cGMP pathway in cerebellum in vivo through activation of AMPA receptors. Neurochem Int 58:599-604.

Bolkan SS, Carvalho Poyraz F, and Kellendonk C (2016) Using human brain imaging studies as a guide toward animal models of schizophrenia. Neuroscience 321:77-98

Bolles RC and Fanselow MS (1980) A perceptual-defensive-recuperative model of fear and pain. Behav Brain Sci 3:291-301.

Bosch F and Rosich L (2008) The contributions of Paul Ehrlich to pharmacology: a tribute on the occasion of the centenary of his Nobel Prize. Pharmacology 82:171-179.

Bosch OJ, Meddle SL, Beiderbeck DI, Douglas AJ, and Neumann ID (2005) Brain oxytocin correlates with maternal aggression: link to anxiety. $J$ Neurosci 25 : $6807-6815$

Boulougouris V, Glennon JC, and Robbins TW (2008) Dissociable effects of selective 5HT2A and 5-HT2C receptor antagonists on serial spatial reversal learning in rats. Neuropsychopharmacology 33:2007-2019.

Boyer P and Liénard P (2006) Why ritualized behavior? Precaution systems and action parsing in developmental, pathological and cultural rituals. Behav Brain Sci 29 $595-613, \mathrm{NaN}-650$.

Brambilla F, Perna G, Bussi R, and Bellodi L (2000) Dopamine function in obsessive compulsive disorder: cortisol response to acute apomorphine stimulation. Psychoneuroendocrinology 25:301-310.

Brand SJ, Moller M, and Harvey BH (2015) A review of biomarkers in mood and psychotic disorders: a dissection of clinical vs. preclinical correlates. Curr Neuropharmacol 13:324-368.

Brennan BP, Jensen JE, Perriello C, Pope HG Jr, Jenike MA, Hudson JI, Rauch SL, and Kaufman MJ (2016) Lower posterior cingulate cortex glutathione levels in obsessive-compulsive disorder. Biol Psychiatry Cogn Neurosci Neuroimaging 1: 116-124.

Brennan BP, Wang D, Li M, Perriello C, Ren J, Elias JA, Van Kirk NP, Krompinger JW, Pope HG Jr, Haber SN, et al. (2019) Use of an individual-level approach to identify cortical connectivity biomarkers in obsessive-compulsive disorder. Biol Psychiatry Cogn Neurosci Neuroimaging 4:27-38.

Brimberg L, Benhar I, Mascaro-Blanco A, Alvarez K, Lotan D, Winter C, Klein J, Moses AE, Somnier FE, Leckman JF, et al. (2012) Behavioral, pharmacological, and immunological abnormalities after streptococcal exposure: a novel rat model of Sydenham chorea and related neuropsychiatric disorders. Neuropsychopharmacology 37: 2076-2087.

Brown GM and Martin JB (1974) Corticosterone, prolactin, and growth hormone responses to handling and new environment in the rat. Psychosom Med 36:241-247.

Brown H, Friston K, and Bestmann S (2011) Active inference, attention, and motor preparation. Front Psychol 2:218.

Brown LT, Mikell CB, Youngerman BE, Zhang Y, McKhann GM 2nd, and Sheth SA (2016) Dorsal anterior cingulotomy and anterior capsulotomy for severe, refractory obsessive-compulsive disorder: a systematic review of observational studies. $J \mathrm{Neu}$ rosurg 124:77-89.

Brown SA and Azzi A (2013) Peripheral circadian oscillators in mammals. Handb Exp Pharmacol 217:45-66.

Brunelin J, Mondino M, Bation R, Palm U, Saoud M, and Poulet E (2018) Transcranial direct current stimulation for obsessive-compulsive disorder: a systematic review. Brain Sci 8:37.

Buijs RM, Scheer FA, Kreier F, Yi C, Bos N, Goncharuk VD, and Kalsbeek A (2006) Organization of circadian functions: interaction with the body. Prog Brain Res 153: 341-360.

Burguière E, Monteiro P, Feng G, and Graybiel AM (2013) Optogenetic stimulation of lateral orbitofronto-striatal pathway suppresses compulsive behaviors. Science 340: 1243-1246.

Burton FH (2017) Back to the future: circuit-testing TS \& OCD. $J$ Neurosci Methods 292:2-11.

Camfield DA, Sarris J, and Berk M (2011) Nutraceuticals in the treatment of obsessive compulsive disorder (OCD): a review of mechanistic and clinical evidence. Prog Neuropsychopharmacol Biol Psychiatry 35:887-895.

Campbell KM, de Lecea L, Severynse DM, Caron MG, McGrath MJ, Sparber SB, Sun LY, and Burton FH (1999) OCD-like behaviors caused by a neuropotentiating transgene targeted to cortical and limbic D1+ neurons. J Neurosci 19:5044-5053.

Cannon WB (1927) Bodily Changes in Pain, Hunger, Fear, and Rage: An Account of Recent Researches into the Function of Emotional Excitement, D. Appleton and Company, New York.

Cano-Ramírez H and Hoffman KL (2017) Activation of the orbitofrontal and anterior cingulate cortices during the expression of a naturalistic compulsive-like behavior in the rabbit. Behav Brain Res 320:67-74.

Cano-Ramírez H and Hoffman KL (2018) Activation of cortical and striatal regions during the expression of a naturalistic compulsive-like behavior in the rabbit. Behav Brain Res 351:168-177.

Carey PD, Warwick J, Harvey BH, Stein DJ, and Seedat S (2004) Single photon emission computed tomography (SPECT) in obsessive-compulsive disorder before and after treatment with inositol. Metab Brain Dis 19:125-134.

Carhart-Harris RL and Friston KJ (2019) REBUS and the narchic brain: toward a unified model of the brain action of psychedelics. Pharmacol Rev 71:316-344.

Carmi L, Alyagon U, Barnea-Ygael N, Zohar J, Dar R, and Zangen A (2018) Clinical and electrophysiological outcomes of deep TMS over the medial prefrontal and anterior cingulate cortices in OCD patients. Brain Stimul 11:158-165.

Casas M, Alvarez E, Duro P, Garcia-Ribera C, Udina C, Velat A, Abella D, RodriguezEspinosa J, Salva P, and Jané F (1986) Antiandrogenic treatment of obsessivecompulsive neurosis. Acta Psychiatr Scand 73:221-222.

Casey BJ, Craddock N, Cuthbert BN, Hyman SE, Lee FS, and Ressler KJ (2013) DSM5 and RDoC: progress in psychiatry research? Nat Rev Neurosci 14:810-814. 
Castrén E and Rantamäki T (2010) The role of BDNF and its receptors in depression and antidepressant drug action: reactivation of developmental plasticity. Dev Neurobiol 70:289-297.

Catapano F, Monteleone P, Fuschino A, Maj M, and Kemali D (1992) Melatonin and cortisol secretion in patients with primary obsessive-compulsive disorder. Psychiatry Res 44:217-225.

Catapano F, Monteleone P, Maj M, and Kemali D (1990) Dexamethasone suppression test in patients with primary obsessive-compulsive disorder and in healthy controls. Neuropsychobiology 23:53-56.

Cermakian N and Boivin DB (2009) The regulation of central and peripheral circadian clocks in humans. Obes Rev 10 (Suppl 2):25-36.

Chaiseha Y, Kang SW, Leclerc B, Kosonsiriluk S, Sartsoongnoen N, and El Halawani ME (2010) Serotonin receptor subtypes influence prolactin secretion in the turkey. Gen Comp Endocrinol 165:170-175.

Chakraborty S, Singh OP, Dasgupta A, Mandal N, and Nath Das H (2009) Correlation between lipid peroxidation-induced TBARS level and disease severity in obsessivecompulsive disorder. Prog Neuropsychopharmacol Biol Psychiatry 33:363-366.

Chamberlain SR, Blackwell AD, Fineberg NA, Robbins TW, and Sahakian BJ (2005) The neuropsychology of obsessive compulsive disorder: the importance of failures in cognitive and behavioural inhibition as candidate endophenotypic markers. Neurosci Biobehav Rev 29:399-419.

Chamberlain SR, Fineberg NA, Menzies LA, Blackwell AD, Bullmore ET, Robbins TW, and Sahakian BJ (2007) Impaired cognitive flexibility and motor inhibition in unaffected first-degree relatives of patients with obsessive-compulsive disorder. $A m$ $J$ Psychiatry 164:335-338.

Chambers CD, Garavan H, and Bellgrove MA (2009) Insights into the neural basis of response inhibition from cognitive and clinical neuroscience. Neurosci Biobehav Rev 33:631-646.

Champagne F, Diorio J, Sharma S, and Meaney MJ (2001) Naturally occurring variations in maternal behavior in the rat are associated with differences in estrogeninducible central oxytocin receptors. Proc Natl Acad Sci USA 98:12736-12741.

Chang K, Frankovich J, Cooperstock M, Cunningham MW, Latimer ME, Murphy TK, Pasternack M, Thienemann M, Williams K, Walter J, et al.; PANS Collaborative Consortium (2015) Clinical evaluation of youth with pediatric acute-onset neuropsychiatric syndrome (PANS): recommendations from the 2013 PANS Consensus Conference. J Child Adolesc Psychopharmacol 25:3-13.

Chappell P, Leckman J, Goodman W, Bissette G, Pauls D, Anderson G, Riddle M, Scahill L, McDougle C, and Cohen D (1996) Elevated cerebrospinal fluid corticotropin-releasing factor in Tourette's syndrome: comparison to obsessive compulsive disorder and normal controls. Biol Psychiatry 39:776-783.

Charnay Y and Léger L (2010) Brain serotonergic circuitries. Dialogues Clin Neurosci 12:471-487.

Charney DS, Goodman WK, Price LH, Woods SW, Rasmussen SA, and Heninger GR (1988) Serotonin function in obsessive-compulsive disorder. A comparison of the effects of tryptophan and m-chlorophenylpiperazine in patients and healthy subjects. Arch Gen Psychiatry 45:177-185.

Chasson GS, Buhlmann U, Tolin DF, Rao SR, Reese HE, Rowley T, Welsh KS, and Wilhelm S (2010) Need for speed: evaluating slopes of OCD recovery in behavior therapy enhanced with d-cycloserine. Behav Res Ther 48:675-679.

Chaudron LH and Nirodi N (2010) The obsessive-compulsive spectrum in the perinatal period: a prospective pilot study. Arch Women Ment Health 13:403-410.

Chen SK, Tvrdik P, Peden E, Cho S, Wu S, Spangrude G, and Capecchi MR (2010) Hematopoietic origin of pathological grooming in Hoxb8 mutant mice. Cell 141 775-785

Chimakurthy $\mathrm{J}$ and Murthy TE (2010) Effect of curcumin on quinpirole induced compulsive checking: an approach to determine the predictive and construct validity of the model. $N$ Am $J$ Med Sci 2:81-86.

Chiocca EA and Martuza RL (1990) Neurosurgical therapy of obsessive-compulsive disorder, in Obsessive-Compulsive Disorders: Theory and Management (Jenike MA, Baer L, and Minichiello WE eds) pp 283-294, Year Book Medical Publishers Inc, Chicago, IL.

Chiu S, Gericke N, Farina-Woodbury M, Badmaev V, Raheb H, Terpstra K, Antongiorgi J, Bureau Y, Cernovsky Z, Hou J, et al. (2014) Proof-of-concept randomized controlled study of cognition effects of the proprietary extract Sceletium tortuosum (zembrin) targeting phosphodiesterase- 4 in cognitively healthy subjects: implications for Alzheimer's dementia. Evid Based Complement Alternat Med 2014:682014.

Chomsky N (1959) A review of BF skinner's verbal behavior. Language 35:26-58

Chou-Green JM, Holscher TD, Dallman MF, and Akana SF (2003a) Compulsive behavior in the 5-HT2C receptor knockout mouse. Physiol Behav 78:641-649.

Chou-Green JM, Holscher TD, Dallman MF, and Akana SF (2003b) Repeated stress in young and old 5-HT(2C) receptor knockout mice. Physiol Behav 79:217-226.

Chouinard G, Bélanger MC, Beauclair L, Sultan S, and Murphy BE (1996) Potentiation of fluoxetine by aminoglutethimide, an adrenal steroid suppressant, in obsessive-compulsive disorder resistant to SSRIs: a case report. Prog Neuropsychopharmacol Biol Psychiatry 20:1067-1079.

Clark DM (2018) Realizing the mass public benefit of evidence-based psychological therapies: the IAPT program. Annu Rev Clin Psychol 14:159-183.

Clifton PG (2017) Neural circuits of eating behaviour: opportunities for therapeutic development. J Psychopharmacol 31:1388-1402.

Clomipramine Collaborative Study Group (1991) Clomipramine in the treatment of patients with obsessive-compulsive disorder. Arch Gen Psychiatry 48:730-738.

Cochran DM, Fallon D, Hill M, and Frazier JA (2013) The role of oxytocin in psychiatric disorders: a review of biological and therapeutic research findings. Harv Rev Psychiatry 21:219-247.

Collier G and Johnson DF (2004) The paradox of satiation. Physiol Behav 82:149-153. Collins SM, Surette M, and Bercik P (2012) The interplay between the intestinal microbiota and the brain. Nat Rev Microbiol 10:735-742.

Compagnone NA and Mellon SH (2000) Neurosteroids: biosynthesis and function of these novel neuromodulators. Front Neuroendocrinol 21:1-56.
Corbit VL, Manning EE, Gittis AH, and Ahmari SE (2019) Strengthened inputs from secondary motor cortex to striatum in a mouse model of compulsive behavior $J$ Neurosci 39:2965-2975.

Cotman CW and Berchtold NC (2002) Exercise: a behavioral intervention to enhance brain health and plasticity. Trends Neurosci 25:295-301.

Cottraux J, Gérard D, Cinotti L, Froment JC, Deiber MP, Le Bars D, Galy G, Millet P, Labbé C, Lavenne F, et al. (1996) A controlled positron emission tomography study of obsessive and neutral auditory stimulation in obsessive-compulsive disorder with checking rituals. Psychiatry Res 60:101-112.

Cottraux J, Note I, Yao SN, Lafont S, Note B, Mollard E, Bouvard M, Sauteraud A, Bourgeois M, and Dartigues JF (2001) A randomized controlled trial of cognitive therapy versus intensive behavior therapy in obsessive compulsive disorder. Psychother Psychosom 70:288-297.

Cottraux JA, Bouvard M, Claustrat B, and Juenet C (1984) Abnormal dexamethasone suppression test in primary obsessive-compulsive patients: a confirmatory report. Psychiatry Res 13:157-165.

Couto JP and Moreira R (2018) Oral N-acetylcysteine in the treatment of obsessivecompulsive disorder: a systematic review of the clinical evidence. Prog Neuropsychopharmacol Biol Psychiatry 86:245-254.

Cummings JL and Cunningham K (1992) Obsessive-compulsive disorder in Huntington's disease. Biol Psychiatry 31:263-270.

Cunningham NR, Tran ST, Lynch-Jordan AM, Ting TV, Sil S, Strotman D, Noll JG, Powers SW, Arnold LM, and Kashikar-Zuck S (2015) Psychiatric disorders in young adults diagnosed with juvenile fibromyalgia in adolescence. J Rheumatol 42 2427-2433.

Cuthbert BN (2014) The RDoC framework: facilitating transition from ICD/DSM to dimensional approaches that integrate neuroscience and psychopathology. World Psychiatry 13:28-35.

Dalley JW, Everitt BJ, and Robbins TW (2011) Impulsivity, compulsivity, and topdown cognitive control. Neuron 69:680-694.

Dallmann R, Brown SA, and Gachon F (2014) Chronopharmacology: new insights and therapeutic implications. Annu Rev Pharmacol Toxicol 54:339-361.

Darby RR, Laganiere S, Pascual-Leone A, Prasad S, and Fox MD (2017) Finding the imposter: brain connectivity of lesions causing delusional misidentifications. Brain 140:497-507.

da Rocha FF and Correa H (2011) Is circadian rhythm disruption important in obsessive-compulsive disorder (OCD)? A case of successful augmentation with agomelatine for the treatment of OCD. Clin Neuropharmacol 34:139-140.

da Rocha FF, Correa H, and Teixeira AL (2008) Obsessive-compulsive disorder and immunology: a review. Prog Neuropsychopharmacol Biol Psychiatry 32:1139-1146. Davis BA and Isles AR (2014) Modelling the genetic contribution to mental illness: a timely end for the psychiatric rodent? Eur J Neurosci 39:1933-1942.

Davis J, Moylan S, Harvey BH, Maes M, and Berk M (2014) Neuroprogression in schizophrenia: pathways underpinning clinical staging and therapeutic corollaries. Aust N Z J Psychiatry 48:512-529.

Davis M, Ressler K, Rothbaum BO, and Richardson R (2006) Effects of D-cycloserine on extinction: translation from preclinical to clinical work. Biol Psychiatry 60:369-375

De Berardis D, Serroni N, Campanella D, Olivieri L, Moschetta FS, Conti CM, Conti P, and Di Giannantonio M (2012) A case of obsessive-compulsive disorder successfully treated with agomelatine monotherapy. J Clin Psychopharmacol 32:289-290.

de Bodinat C, Guardiola-Lemaitre B, Mocaër E, Renard P, Muñoz C, and Millan MJ (2010) Agomelatine, the first melatonergic antidepressant: discovery, characterization and development. Nat Rev Drug Discov 9:628-642.

De Crescenzo F, Perelli F, Armando M, and Vicari S (2014) Selective serotonin reuptake inhibitors (SSRIs) for post-partum depression (PPD): a systematic review of randomized clinical trials. J Affect Disord 152-154:39-44.

Deczkowska A, Amit I, and Schwartz M (2018) Microglial immune checkpoint mechanisms. Nat Neurosci 21:779-786.

de Leeuw AS, van Megen HJ, Kahn RS, and Westenberg HG (2017) d-cycloserine addition to exposure sessions in the treatment of patients with obsessive-compulsive disorder. Eur Psychiatry 40:38-44.

Dell'Osso B, Benatti B, Hollander E, Fineberg N, Stein DJ, Lochner C, Nicolini H, Lanzagorta N, Palazzo C, Altamura AC, et al. (2016) Childhood, adolescent and adult age at onset and related clinical correlates in obsessive-compulsive disorder: a report from the International College of Obsessive-Compulsive Spectrum Disorders (ICOCS). Int J Psychiatry Clin Pract 20:210-217.

Delport A, Harvey BH, Petzer A, and Petzer JP (2017) Methylene blue and its analogues as antidepressant compounds. Metab Brain Dis 32:1357-1382.

Delport A, Harvey BH, Petzer A, and Petzer JP (2018) Methylene blue analogues with marginal monoamine oxidase inhibition retain antidepressant-like activity. ACS Chem Neurosci 9:2917-2928.

den Boer JA and Westenberg HG (1992) Oxytocin in obsessive compulsive disorder. Peptides 13:1083-1085.

Denton D, Shade R, Zamarippa F, Egan G, Blair-West J, McKinley M, Lancaster J, and Fox P (1999) Neuroimaging of genesis and satiation of thirst and an interoceptor-driven theory of origins of primary consciousness. Proc Natl Acad Sci USA 96:5304-5309.

Denys D (2006) Pharmacotherapy of obsessive-compulsive disorder and obsessivecompulsive spectrum disorders. Psychiatr Clin North Am 29:553-584, xi.

Denys D, Fluitman S, Kavelaars A, Heijnen C, and Westenberg H (2004a) Decreased TNF-alpha and NK activity in obsessive-compulsive disorder. Psychoneuroendocrinology 29:945-952.

Denys D, Mantione M, Figee M, van den Munckhof P, Koerselman F, Westenberg H, Bosch A, and Schuurman R (2010) Deep brain stimulation of the nucleus accumbens for treatment-refractory obsessive-compulsive disorder. Arch Gen Psychiatry 67 1061-1068.

Denys D, van der Wee N, Janssen J, De Geus F, and Westenberg HG (2004b) Low level of dopaminergic D2 receptor binding in obsessive-compulsive disorder. Biol Psychiatry 55:1041-1045 
Denys D, Van Nieuwerburgh F, Deforce D, and Westenberg H (2006) Association between the dopamine D2 receptor TaqI A2 allele and low activity COMT allele with obsessive-compulsive disorder in males. Eur Neuropsychopharmacol 16 : 446-450.

Denys D, Zohar J, and Westenberg HG (2004c) The role of dopamine in obsessivecompulsive disorder: preclinical and clinical evidence. J Clin Psychiatry 65 (Suppl 14):11-17.

Desbonnet L, Clarke G, Traplin A, O’Sullivan O, Crispie F, Moloney RD, Cotter PD, Dinan TG, and Cryan JF (2015) Gut microbiota depletion from early adolescence in mice: implications for brain and behaviour. Brain Behav Immun 48:165-173.

Desbonnet L, Garrett L, Clarke G, Bienenstock J, and Dinan TG (2008) The probiotic Bifidobacteria infantis: an assessment of potential antidepressant properties in the rat. J Psychiatr Res 43:164-174.

de Wit SJ, Alonso P, Schweren L, Mataix-Cols D, Lochner C, Menchón JM, Stein DJ, Fouche JP, Soriano-Mas C, Sato JR, et al. (2014) Multicenter voxel-based morphometry mega-analysis of structural brain scans in obsessive-compulsive disorder. Am J Psychiatry 171:340-349.

Diaz Heijtz R, Wang S, Anuar F, Qian Y, Björkholm B, Samuelsson A, Hibberd ML, Forssberg H, and Pettersson S (2011) Normal gut microbiota modulates brain development and behavior. Proc Natl Acad Sci USA 108:3047-3052.

Dibner C, Schibler U, and Albrecht U (2010) The mammalian circadian timing system: organization and coordination of central and peripheral clocks. Annu Rev Physiol 72: $517-549$

Di Giovanni G, Di Matteo V, Pierucci M, and Esposito E (2008) Serotonin-dopamine interaction: electrophysiological evidence, in Serotonin-Dopamine Interaction: Ex perimental Evidence and Therapeutic Relevance (DiGiovanni G, DiMatteo V, and Esposito E eds) pp 45-71, Elsevier Science B.V., Amsterdam.

Dillon KM and Brooks D (1992) Unusual cleaning behavior in the luteal phase. Psychol Rep 70:35-39.

Dinan TG and Cryan JF (2017) Gut-brain axis in 2016: brain-gut-microbiota axis mood, metabolism and behaviour. Nat Rev Gastroenterol Hepatol 14:69-70.

Djodari-Irani A, Klein J, Banzhaf J, Joel D, Heinz A, Harnack D, Lagemann T, Juckel G, Kupsch A, Morgenstern R, et al. (2011) Activity modulation of the globus pallidus and the nucleus entopeduncularis affects compulsive checking in rats. Behav Brain Res 219:149-158.

Dobzhansky T (1973) Nothing in biology makes sense except in the light of evolution. Am Biol Teach 35:125-129.

Dolan KE, Finley HJ, Burns CM, Gasta MG, Gossard CM, Parker EC, Pizano JM, Williamson CB, and Lipski EA (2016) Probiotics and disease: a comprehensive summary-part 1, mental and neurological health. Integr Med (Encinitas) 15:46-58

Dold M, Aigner M, Lanzenberger R, and Kasper S (2013) Antipsychotic augmentation of serotonin reuptake inhibitors in treatment-resistant obsessive-compulsive disorder: a meta-analysis of double-blind, randomized, placebo-controlled trials. Int $J$ Neuropsychopharmacol 16:557-574.

Dold M, Aigner M, Lanzenberger R, and Kasper S (2015) Antipsychotic augmentation of serotonin reuptake inhibitors in treatment-resistant obsessive-compulsive disorder: an update meta-analysis of double-blind, randomized, placebo-controlled trials. Int J Neuropsychopharmacol 18:pyv047.

Dougherty DD, Baer L, Cosgrove GR, Cassem EH, Price BH, Nierenberg AA, Jenike MA, and Rauch SL (2002) Prospective long-term follow-up of 44 patients who received cingulotomy for treatment-refractory obsessive-compulsive disorder. Am J Psychiatry 159:269-275.

Dreyer JL (2010) New insights into the roles of microRNAs in drug addiction and neuroplasticity. Genome Med 2:92.

Dubuisson D and Dennis SG (1977) The formalin test: a quantitative study of the analgesic effects of morphine, meperidine, and brain stem stimulation in rats and cats. Pain 4:161-174.

D'Urso G, Brunoni AR, Mazzaferro MP, Anastasia A, de Bartolomeis A, and Mantovani A (2016) Transcranial direct current stimulation for obsessive-compulsive disorder: a randomized, controlled, partial crossover trial. Depress Anxiety 33:1132-1140.

du Toit PL, van Kradenburg J, Niehaus D, and Stein DJ (2001) Comparison of obsessive-compulsive disorder patients with and without comorbid putative obsessive-compulsive spectrum disorders using a structured clinical interview. Compr Psychiatry 42:291-300.

Dvorkin A, Perreault ML, and Szechtman H (2006) Development and temporal organization of compulsive checking induced by repeated injections of the dopamine agonist quinpirole in an animal model of obsessive-compulsive disorder. Behav Brain Res 169:303-311.

Dvorkin A, Silva C, McMurran T, Bisnaire L, Foster J, and Szechtman H (2010) Features of compulsive checking behavior mediated by nucleus accumbens and orbital frontal cortex. Eur J Neurosci 32:1552-1563.

Dyster TG, Mikell CB, and Sheth SA (2016) The co-evolution of neuroimaging and psychiatric neurosurgery. Front Neuroanat 10:68.

Eagle DM and Baunez C (2010) Is there an inhibitory-response-control system in the rat? Evidence from anatomical and pharmacological studies of behavioral inhibition. Neurosci Biobehav Rev 34:50-72.

Eagle DM, Lehmann O, Theobald DE, Pena Y, Zakaria R, Ghosh R, Dalley JW, and Robbins TW (2009) Serotonin depletion impairs waiting but not stop-signal reaction time in rats: implications for theories of the role of 5-HT in behavioral inhibition. Neuropsychopharmacology 34:1311-1321.

Eagle DM, Wong JC, Allan ME, Mar AC, Theobald DE, and Robbins TW (2011) Contrasting roles for dopamine D1 and D2 receptor subtypes in the dorsomedial striatum but not the nucleus accumbens core during behavioral inhibition in the stop-signal task in rats. $J$ Neurosci 31:7349-7356.

Eftimiadi C, Eftimiadi G, and Vinai P (2016) Staphylococcus aureus colonization modulates tic expression and the host immune response in a girl with Tourette syndrome. Front Psychiatry 7:31.

Egashira N, Okuno R, Matsushita M, Abe M, Mishima K, Iwasaki K, Nishimura R, Oishi R, and Fujiwara M (2008) Aripiprazole inhibits marble-burying behavior via 5-hydroxytryptamine (5-HT)1A receptor-independent mechanisms. Eur J Pharmacol 592:103-108.
Eilam D, Golani I, and Szechtman H (1989) D2-agonist quinpirole induces perseveration of routes and hyperactivity but no perseveration of movements. Brain Res 490 $255-267$.

Einat $\mathrm{H}$ and Belmaker RH (2001) The effects of inositol treatment in animal models of psychiatric disorders. J Affect Disord 62:113-121.

Eisen JL, Leonard HL, Swedo SE, Price LH, Zabriskie JB, Chiang SY, Karitani M, and Rasmussen SA (2001) The use of antibody D8/17 to identify B cells in adults with obsessive-compulsive disorder. Psychiatry Res 104:221-225.

El Mansari M and Blier P (2006) Mechanisms of action of current and potential pharmacotherapies of obsessive-compulsive disorder. Prog Neuropsychopharmacol Biol Psychiatry 30:362-373.

Emamzadehfard S, Kamaloo A, Paydary K, Ahmadipour A, Zeinoddini A, Ghaleiha A Mohammadinejad P, Zeinoddini A, and Akhondzadeh S (2016) Riluzole in augmentation of fluvoxamine for moderate to severe obsessive-compulsive disorder: randomized, double-blind, placebo-controlled study. Psychiatry Clin Neurosci 70:332-341.

Emmelkamp PM and Kwee KG (1977) Obsessional ruminations: a comparison between thought-stopping and prolonged exposure in imagination. Behav Res Ther 15: 441-444.

Epperson CN, McDougle CJ, and Price LH (1996) Intranasal oxytocin in obsessivecompulsive disorder. Biol Psychiatry 40:547-549.

Ercan TE, Ercan G, Severge B, Arpaozu M, and Karasu G (2008) Mycoplasma pneumoniae infection and obsessive-compulsive disease: a case report. J Child Neurol 23: 338-340.

Eriksson E, Hedberg MA, Andersch B, and Sundblad C (1995) The serotonin reuptake inhibitor paroxetin is superior to the noradrenaline reuptake inhibitor maprotiline in the treatment of premenstrual syndrome. Neuropsychopharmacology 12:167-176.

Ersan S, Bakir S, Erdal Ersan E, and Dogan O (2006) Examination of free radical metabolism and antioxidant defence system elements in patients with obsessivecompulsive disorder. Prog Neuropsychopharmacol Biol Psychiatry 30:1039-1042.

Esalatmanesh S, Abrishami Z, Zeinoddini A, Rahiminejad F, Sadeghi M, Najarzadegan MR, Shalbafan MR, and Akhondzadeh S (2016) Minocycline combination therapy with fluvoxamine in moderate-to-severe obsessive-compulsive disorder: a placebo-controlled, double-blind, randomized trial. Psychiatry Clin Neurosci 70: $517-526$.

Färber K, Pannasch U, and Kettenmann H (2005) Dopamine and noradrenaline control distinct functions in rodent microglial cells. Mol Cell Neurosci 29:128-138. Farrell LJ, Waters AM, Boschen MJ, Hattingh L, McConnell H, Milliner EL, Collings N, Zimmer-Gembeck M, Shelton D, Ollendick TH, et al. (2013) Difficult-to-treat pediatric obsessive-compulsive disorder: feasibility and preliminary results of a randomized pilot trial of D-cycloserine-augmented behavior therapy. Depress Anxiety 30: 723-731.

Farris SG, McLean CP, Van Meter PE, Simpson HB, and Foa EB (2013) Treatment response, symptom remission, and wellness in obsessive-compulsive disorder. J Clin Psychiatry 74:685-690.

Felger JC and Treadway MT (2017) Inflammation effects on motivation and motor activity: role of dopamine. Neuropsychopharmacology 42:216-241.

Fernández Córdoba E and López-Ibor Aliño J (1967) Use of monochlorimipramine in psychiatric patients who are resistant to other therapy. Actas Luso Esp Neurol Psiquiatr 26:119-147.

Fernández-Guasti A and Rodríguez-Manzo G (2003) Pharmacological and physiological aspects of sexual exhaustion in male rats. Scand $J$ Psychol 44:257-263.

Fessel JP and Oldham WM (2018) Pyridine dinucleotides from molecules to man. Antioxid Redox Signal 28:180-212.

Feygin DL, Swain JE, and Leckman JF (2006) The normalcy of neurosis: evolutionary origins of obsessive-compulsive disorder and related behaviors. Prog Neuropsychopharmacol Biol Psychiatry 30:854-864.

Fidalgo S, Ivanov DK, and Wood SH (2013) Serotonin: from top to bottom. Biogerontology 14:21-45

Figee M, Wielaard I, Mazaheri A, and Denys D (2013) Neurosurgical targets for compulsivity: what can we learn from acquired brain lesions? Neurosci Biobehav Rev 37:328-339.

Findley DB, Leckman JF, Katsovich L, Lin H, Zhang H, Grantz H, Otka J, Lombroso PJ, and King RA (2003) Development of the Yale Children's Global Stress Index (YCGSI) and its application in children and adolescents with Tourette's syndrome and obsessive-compulsive disorder. J Am Acad Child Adolesc Psychiatry 42:450-457.

Fineberg NA, Gale TM, and Sivakumaran T (2006) A review of antipsychotics in the treatment of obsessive compulsive disorder. J Psychopharmacol 20:97-103.

Fineberg NA, Reghunandanan S, Simpson HB, Phillips KA, Richter MA, Matthews K, Stein DJ, Sareen J, Brown A, and Sookman D; Accreditation Task Force of The Canadian Institute for Obsessive Compulsive Disorders (2015) Obsessive-compulsive disorder (OCD): practical strategies for pharmacological and somatic treatment in adults. Psychiatry Res 227:114-125.

Finkel MS, Laghrissi-Thode F, Pollock BG, and Rong J (1996) Paroxetine is a novel nitric oxide synthase inhibitor. Psychopharmacol Bull 32:653-658.

Fireman B, Koran LM, Leventhal JL, and Jacobson A (2001) The prevalence of clinically recognized obsessive-compulsive disorder in a large health maintenance organization. Am J Psychiatry 158:1904-1910.

First MB (2013) DSM-5 Handbook of Differential Diagnosis, American Psychiatric Publishing, Arlington, VA.

Fisher SK, Heacock AM, and Agranoff BW (1992) Inositol lipids and signal transduction in the nervous system: an update. J Neurochem 58:18-38.

Flegr J and Horáček J (2017) Toxoplasma-infected subjects report an obsessivecompulsive disorder diagnosis more often and score higher in obsessive-compulsive inventory. Eur Psychiatry 40:82-87.

Fleischman DS and Fessler DM (2011) Progesterone's effects on the psychology of disease avoidance: support for the compensatory behavioral prophylaxis hypothesis. Horm Behav 59:271-275.

Fluitman SB, Denys DA, Heijnen CJ, and Westenberg HG (2010) Disgust affects TNFalpha, IL-6 and noradrenalin levels in patients with obsessive-compulsive disorder. Psychoneuroendocrinology 35:906-911. 
Foa EB (2010) Cognitive behavioral therapy of obsessive-compulsive disorder. Dialogues Clin Neurosci 12:199-207.

Foa EB, Liebowitz MR, Kozak MJ, Davies S, Campeas R, Franklin ME, Huppert JD Kjernisted K, Rowan V, Schmidt AB, et al. (2005) Randomized, placebo-controlled trial of exposure and ritual prevention, clomipramine, and their combination in the treatment of obsessive-compulsive disorder. Am J Psychiatry 162:151-161.

Foa EB, Steketee G, Grayson JB, Turner RM, and Latimer PR (1984) Deliberate exposure and blocking of obsessive-compulsive rituals: immediate and long-term effects. Behav Ther 15:450-472.

Fontenelle LF, Barbosa IG, Luna JV, de Sousa LP, Abreu MN, and Teixeira AL (2012) A cytokine study of adult patients with obsessive-compulsive disorder. Compr Psychiatry 53:797-804.

Fontenelle LF, Mendlowicz MV, and Versiani M (2005) Clinical subtypes of obsessivecompulsive disorder based on the presence of checking and washing compulsions. $\mathrm{Br}$ J Psychiatry 27:201-207.

Fontenelle LF, Mendlowicz MV, and Versiani M (2006) The descriptive epidemiology of obsessive-compulsive disorder. Prog Neuropsychopharmacol Biol Psychiatry 30: $327-337$.

Fornaro M (2011) Switching from serotonin reuptake inhibitors to agomelatine in patients with refractory obsessive-compulsive disorder: a 3 month follow-up case series. Ann Gen Psychiatry 10:5.

Forray A, Focseneanu M, Pittman B, McDougle CJ, and Epperson CN (2010) Onset and exacerbation of obsessive-compulsive disorder in pregnancy and the postpartum period. J Clin Psychiatry 71:1061-1068.

Fouche JP, du Plessis S, Hattingh C, Roos A, Lochner C, Soriano-Mas C, Sato JR Nakamae T, Nishida S, Kwon JS, et al.; OCD Brain Imaging Consortium (2017) Cortical thickness in obsessive-compulsive disorder: multisite mega-analysis of 780 brain scans from six centres. Br J Psychiatry 210:67-74.

Frank MG, Fonken LK, Watkins LR, and Maier SF (2019) Microglia: Neuroimmunesensors of stress. Semin Cell Dev Biol 94:176-185.

Frederick AL and Stanwood GD (2009) Drugs, biogenic amine targets and the developing brain. Dev Neurosci 31:7-22

Freeman EW, Rickels K, Sondheimer SJ, and Polansky M (1999) Differential response to antidepressants in women with premenstrual syndrome/premenstrual dysphoric disorder: a randomized controlled trial. Arch Gen Psychiatry 56:932-939.

Frey R, Metzler D, Fischer P, Heiden A, Scharfetter J, Moser E, and Kasper S (1998) Myo-inositol in depressive and healthy subjects determined by frontal $1 \mathrm{H}$-magnetic resonance spectroscopy at 1.5 tesla. $J$ Psychiatr Res 32:411-420.

Frick L and Pittenger C (2016) Microglial dysregulation in OCD, Tourette syndrome, and PANDAS. J Immunol Res 2016:8606057.

Friedlander L and Desrocher M (2006) Neuroimaging studies of obsessive-compulsive disorder in adults and children. Clin Psychol Rev 26:32-49.

Furtado M and Katzman MA (2015) Neuroinflammatory pathways in anxiety, posttraumatic stress, and obsessive compulsive disorders. Psychiatry Res 229:37-48.

Fux M, Benjamin J, and Belmaker RH (1999) Inositol versus placebo augmentation of serotonin reuptake inhibitors in the treatment of obsessive-compulsive disorder a double-blind cross-over study. Int $J$ Neuropsychopharmacol 2:193-195.

Fux M, Levine J, Aviv A, and Belmaker RH (1996) Inositol treatment of obsessivecompulsive disorder. Am J Psychiatry 153:1219-1221.

Gabbay V, Coffey BJ, Guttman LE, Gottlieb L, Katz Y, Babb JS, Hamamoto MM, and Gonzalez CJ (2009) A cytokine study in children and adolescents with Tourette's disorder. Prog Neuropsychopharmacol Biol Psychiatry 33:967-971.

Gainetdinov RR (2018) Trace amines and their receptors. Pharmacol Rev 70 (3): 549-620, doi: 10.1124/pr.117.015305 29941461.

Gava I, Barbui C, Aguglia E, Carlino D, Churchill R, De Vanna M, and McGuire HF (2007) Psychological treatments versus treatment as usual for obsessive compulsive disorder (OCD). Cochrane Database Syst Rev (2):CD005333.

Gawali NB, Chowdhury AA, Kothavade PS, Bulani VD, Nagmoti DM, and Juvekar AR (2016) Involvement of nitric oxide in anticompulsive-like effect of agmatine on marble-burying behaviour in mice. Eur J Pharmacol 770:165-171.

Geller D, Biederman J, Jones J, Park K, Schwartz S, Shapiro S, and Coffey B (1998) Is juvenile obsessive-compulsive disorder a developmental subtype of the disorder? A review of the pediatric literature. J Am Acad Child Adolesc Psychiatry 37:420-427.

Geller DA, Biederman J, Faraone S, Agranat A, Cradock K, Hagermoser L, Kim G, Frazier J, and Coffey BJ (2001) Developmental aspects of obsessive compulsive disorder: findings in children, adolescents, and adults. $J$ Nerv Ment Dis 189:471-477.

Geller DA, Biederman J, Griffin S, Jones J, and Lefkowitz TR (1996) Comorbidity of juvenile obsessive-compulsive disorder with disruptive behavior disorders. J Am Acad Child Adolesc Psychiatry 35:1637-1646.

Georgiadou G, Tarantilis PA, and Pitsikas N (2012) Effects of the active constituents of Crocus Sativus L., crocins, in an animal model of obsessive-compulsive disorder. Neurosci Lett 528:27-30.

Ghaleiha A, Entezari N, Modabbernia A, Najand B, Askari N, Tabrizi M, Ashrafi M, Hajiaghaee R, and Akhondzadeh S (2013) Memantine add-on in moderate to severe obsessive-compulsive disorder: randomized double-blind placebo-controlled study. $J$ Psychiatr Res 47:175-180.

Gilbert P (1989) Human Nature and Suffering, Lawrence Erlbaum, London.

Gill SR, Pop M, Deboy RT, Eckburg PB, Turnbaugh PJ, Samuel BS, Gordon JI, Relman DA, Fraser-Liggett CM, and Nelson KE (2006) Metagenomic analysis of the human distal gut microbiome. Science 312:1355-1359.

Glass JD, Grossman GH, Farnbauch L, and DiNardo L (2003) Midbrain raphe modulation of nonphotic circadian clock resetting and 5-HT release in the mammalian suprachiasmatic nucleus. $J$ Neurosci 23:7451-7460.

Gluckman PD, Hanson MA, and Spencer HG (2005) Predictive adaptive responses and human evolution. Trends Ecol Evol 20:527-533.

Goodman WK, Grice DE, Lapidus KA, and Coffey BJ (2014) Obsessive-compulsive disorder. Psychiatr Clin North Am 37:257-267.

Goodman WK, Price LH, Delgado PL, Palumbo J, Krystal JH, Nagy LM, Rasmussen SA, Heninger GR, and Charney DS (1990) Specificity of serotonin reuptake inhibitors in the treatment of obsessive-compulsive disorder. Comparison of fluvoxamine and desipramine. Arch Gen Psychiatry 47:577-585.
Goodman WK, Price LH, Rasmussen SA, Mazure C, Delgado P, Heninger GR and Charney DS (1989a) The Yale-Brown Obsessive Compulsive Scale. II. Validity. Arch Gen Psychiatry 46:1012-1016.

Goodman WK, Price LH, Rasmussen SA, Mazure C, Fleischmann RL, Hill CL, Heninger GR, and Charney DS (1989b) The Yale-Brown Obsessive Compulsive Scale. I. Development, use, and reliability. Arch Gen Psychiatry 46: 1006-1011.

Göttlich M, Krämer UM, Kordon A, Hohagen F, and Zurowski B (2014) Decreased limbic and increased fronto-parietal connectivity in unmedicated patients with obsessive-compulsive disorder. Hum Brain Mapp 35:5617-5632.

Grados MA, Specht MW, Sung HM, and Fortune D (2013) Glutamate drugs and pharmacogenetics of OCD: a pathway-based exploratory approach. Expert Opin Drug Discov 8:1515-1527.

Graeber C (2018) The Breakthrough: Immunotherapy and the Race to Cure Cancer, Grand Central Publishing, New York.

Gray SM and Bloch MH (2012) Systematic review of proinflammatory cytokines in obsessive-compulsive disorder. Curr Psychiatry Rep 14:220-228.

Greenberg BD, Gabriels LA, Malone DA Jr, Rezai AR, Friehs GM, Okun MS, Shapira NA, Foote KD, Cosyns PR, Kubu CS, et al. (2010) Deep brain stimulation of the ventral internal capsule/ventral striatum for obsessive-compulsive disorder: worldwide experience. Mol Psychiatry 15:64-79.

Greenberg BD, George MS, Martin JD, Benjamin J, Schlaepfer TE, Altemus M, Wassermann EM, Post RM, and Murphy DL (1997) Effect of prefrontal repetitive transcranial magnetic stimulation in obsessive-compulsive disorder: a preliminary study. Am J Psychiatry 154:867-869.

Greene-Schloesser DM, Van der Zee EA, Sheppard DK, Castillo MR, Gregg KA Burrow T, Foltz H, Slater M, and Bult-Ito A (2011) Predictive validity of a noninduced mouse model of compulsive-like behavior. Behav Brain Res 221:55-62.

Greer JM and Capecchi MR (2002) Hoxb8 is required for normal grooming behavior in mice. Neuron 33:23-34.

Greist JH (1992) An integrated approach to treatment of obsessive compulsive disorder. J Clin Psychiatry 53 (Suppl):38-41.

Greist JH, Jefferson JW, Kobak KA, Katzelnick DJ, and Serlin RC (1995) Efficacy and tolerability of serotonin transport inhibitors in obsessive-compulsive disorder. A meta-analysis. Arch Gen Psychiatry 52:53-60.

Grillault Laroche D and Gaillard A (2016) Induced obsessive compulsive symptoms (OCS) in schizophrenia patients under atypical 2 antipsychotics (AAPs): review and hypotheses. Psychiatry Res 246:119-128.

Groër MW (2005) Differences between exclusive breastfeeders, formula-feeders, and controls: a study of stress, mood, and endocrine variables. Biol Res Nurs 7:106-117.

Groman SM, James AS, and Jentsch JD (2009) Poor response inhibition: at the nexus between substance abuse and attention deficit/hyperactivity disorder. Neurosci Biobehav Rev 33:690-698.

Gross-Isseroff R, Cohen R, Sasson Y, Voet H, and Zohar J (2004) Serotonergic dissection of obsessive compulsive symptoms: a challenge study with m-chlorophenylpiperazine and sumatriptan. Neuropsychobiology 50:200-205.

Gruninger TR, LeBoeuf B, Liu Y, and Garcia LR (2007) Molecular signaling involved in regulating feeding and other motivated behaviors. Mol Neurobiol 35:1-20.

Guardiola-Lemaitre B, De Bodinat C, Delagrange P, Millan MJ, Munoz C, and Mocaër E (2014) Agomelatine: mechanism of action and pharmacological profile in relation to antidepressant properties. Br J Pharmacol 171:3604-3619.

Guastella AJ, Richardson R, Lovibond PF, Rapee RM, Gaston JE, Mitchell P, and Dadds MR (2008) A randomized controlled trial of D-cycloserine enhancement of exposure therapy for social anxiety disorder. Biol Psychiatry 63:544-549.

Guglielmi V, Vulink NC, Denys D, Wang Y, Samuels JF, and Nestadt G (2014) Obsessive-compulsive disorder and female reproductive cycle events: results from the OCD and reproduction collaborative study. Depress Anxiety 31:979-987.

Güldenpfennig M, Wolmarans W, du Preez JL, Stein DJ, and Harvey BH (2011) Cortico-striatal oxidative status, dopamine turnover and relation with stereotypy in the deer mouse. Physiol Behav 103:404-411.

Guo W, Samuels JF, Wang Y, Cao H, Ritter M, Nestadt PS, Krasnow J, Greenberg BD, Fyer AJ, McCracken JT, et al. (2017) Polygenic risk score and heritability estimates reveals a genetic relationship between ASD and OCD. Eur Neuropsychopharmacol 27:657-666.

Gürsel DA, Avram M, Sorg C, Brandl F, and Koch K (2018) Frontoparietal areas link impairments of large-scale intrinsic brain networks with aberrant fronto-striata interactions in OCD: a meta-analysis of resting-state functional connectivity. $\mathrm{Neu}$ rosci Biobehav Rev 87:151-160.

Gustafsson PE, Gustafsson PA, Ivarsson T, and Nelson N (2008) Diurnal cortisol levels and cortisol response in youths with obsessive-compulsive disorder. Neuropsychobiology 57:14-21.

Hahn-Holbrook J, Holbrook C, and Haselton MG (2011) Parental precaution: neurobiological means and adaptive ends. Neurosci Biobehav Rev 35:1052-1066.

Halberstadt AL, van der Heijden I, Ruderman MA, Risbrough VB, Gingrich JA, Geyer MA, and Powell SB (2009) 5-HT(2A) and 5-HT(2C) receptors exert opposing effects on locomotor activity in mice. Neuropsychopharmacology 34:1958-1967.

Hall D, Dhilla A, Charalambous A, Gogos JA, and Karayiorgou M (2003) Sequence variants of the brain-derived neurotrophic factor (BDNF) gene are strongly associated with obsessive-compulsive disorder. Am J Hum Genet 73:370-376.

Hallett M (2007) Transcranial magnetic stimulation: a primer. Neuron 55:187-199.

Hamani C, Pilitsis J, Rughani AI, Rosenow JM, Patil PG, Slavin KS, Abosch A Eskandar E, Mitchell LS, and Kalkanis S; American Society for Stereotactic and Functional NeurosurgeryConoress of Neurological SurgeonsCNS and American Association of Neurological Surgeons (2014) Deep brain stimulation for obsessivecompulsive disorder: systematic review and evidence-based guideline sponsored by the American Society for Stereotactic and Functional Neurosurgery and the Congress of Neurological Surgeons (CNS) and endorsed by the CNS and American Association of Neurological Surgeons. Neurosurgery 75:327-333, quiz 333.

Hanisch UK and Kettenmann H (2007) Microglia: active sensor and versatile effector cells in the normal and pathologic brain. Nat Neurosci 10:1387-1394. 
Hansen B, Hagen K, Öst LG, Solem S, and Kvale G (2018) The Bergen 4-day OCD treatment delivered in a group setting: 12-month follow-up. Front Psychol 9:639.

Hao H, Chen C, Mao W, Xia W, Yi Z, Zhao P, Pan P, and Dai Z (2019) Alterations in resting-state local functional connectivity in obsessive-compulsive disorder. $J$ Affect Disord 245:113-119.

Hart BL (1988) Biological basis of the behavior of sick animals. Neurosci Biobehav Rev 12:123-137.

Hart BL (1990) Behavioral adaptations to pathogens and parasites: five strategies. Neurosci Biobehav Rev 14:273-294.

Harvey BH (1997) The neurobiology and pharmacology of depression. A comparative overview of serotonin selective antidepressants. S Afr Med J 87 (Suppl):540-550, 552.

Harvey BH, Brink CB, Seedat S, and Stein DJ (2002) Defining the neuromolecular action of myo-inositol: application to obsessive-compulsive disorder. Prog Neuropsychopharmacol Biol Psychiatry 26:21-32.

Harvey BH, Naciti C, Brand L, and Stein DJ (2003) Endocrine, cognitive and hippocampal/cortical $5 \mathrm{HT} 1 \mathrm{~A} / 2 \mathrm{~A}$ receptor changes evoked by a time-dependent sensitisation (TDS) stress model in rats. Brain Res 983:97-107.

Harvey BH, Oosthuizen F, Brand L, Wegener G, and Stein DJ (2004) Stress-restress evokes sustained iNOS activity and altered GABA levels and NMDA receptors in rat hippocampus. Psychopharmacology (Berl) 175:494-502.

Harvey BH, Scheepers A, Brand L, and Stein DJ (2001) Chronic inositol increases striatal $\mathrm{D}(2)$ receptors but does not modify dexamphetamine-induced motor behavior. Relevance to obsessive-compulsive disorder. Pharmacol Biochem Behav 68 245-253.

Harvey BH and Slabbert FN (2014) New insights on the antidepressant discontinuation syndrome. Hum Psychopharmacol 29:503-516.

Harvey BH, Stein DJ, and Emsley RA (1999) The new-generation antipsychoticsintegrating the neuropathology and pharmacology of schizophrenia. S Afr Med J 89 $661-672$

Hastings MH, Brancaccio M, and Maywood ES (2014) Circadian pacemaking in cells and circuits of the suprachiasmatic nucleus. J Neuroendocrinol 26:2-10.

Hastings MH, Maywood ES, and Brancaccio M (2018) Generation of circadian rhythms in the suprachiasmatic nucleus. Nat Rev Neurosci 19:453-469.

Hastings MH, Reddy AB, and Maywood ES (2003) A clockwork web: circadian timing in brain and periphery, in health and disease. Nat Rev Neurosci 4:649-661.

Hazari N, Narayanaswamy JC, and Venkatasubramanian G (2019) Neuroimaging findings in obsessive-compulsive disorder: a narrative review to elucidate neurobiological underpinnings. Indian J Psychiatry 61 (Suppl 1):S9-S29.

He Y, Janssen WG, Rothstein JD, and Morrison JH (2000) Differential synaptic localization of the glutamate transporter EAAC1 and glutamate receptor subunit GluR2 in the rat hippocampus. J Comp Neurol 418:255-269.

Hebb DO (1966) A Textbook of Psychology, Saunders, Philadelphia

Hediger MA, Clémençon B, Burrier RE, and Bruford EA (2013) The ABCs of membrane transporters in health and disease (SLC series): introduction. Mol Aspects Med 34:95-107.

Hemmings SM, Kinnear CJ, Van der Merwe L, Lochner C, Corfield VA, MoolmanSmook JC, and Stein DJ (2008) Investigating the role of the brain-derived neurotrophic factor (BDNF) val66met variant in obsessive-compulsive disorder (OCD). World J Biol Psychiatry 9:126-134.

Hengartner MP and Lehmann SN (2017) Why psychiatric research must abandon traditional diagnostic classification and adopt a fully dimensional scope: two solutions to a persistent problem. Front Psychiatry 8:101.

Hennessy MB and Levine S (1978) Sensitive pituitary-adrenal responsiveness to varying intensities of psychological stimulation. Physiol Behav 21:295-297.

Hensler JG, Cervera LS, Miller HA, and Corbitt J (1996) Expression and modulation of 5-hydroxytryptamine1A receptors in P11 cells. J Pharmacol Exp Ther 278: 1138-1145.

Hesse S, Müller U, Lincke T, Barthel H, Villmann T, Angermeyer MC, Sabri O, and Stengler-Wenzke K (2005) Serotonin and dopamine transporter imaging in patients with obsessive-compulsive disorder. Psychiatry Res 140:63-72.

Hewitt KN, Lee MD, Dourish CT, and Clifton PG (2002) Serotonin 2C receptor agonists and the behavioural satiety sequence in mice. Pharmacol Biochem Behav $\mathbf{7 1}$ : 691-700.

Hickey M, Bryant C, and Judd F (2012) Evaluation and management of depressive and anxiety symptoms in midlife. Climacteric 15:3-9.

Hickman SE, Kingery ND, Ohsumi TK, Borowsky ML, Wang LC, Means TK, and El Khoury J (2013) The microglial sensome revealed by direct RNA sequencing. Nat Neurosci 16:1896-1905.

Hinds AL, Woody EZ, Drandic A, Schmidt LA, Van Ameringen M, Coroneos M, and Szechtman H (2010) The psychology of potential threat: properties of the security motivation system. Biol Psychol 85:331-337.

Hinds AL, Woody EZ, Schmidt LA, Van Ameringen M, and Szechtman H (2015) In the wake of a possible mistake: security motivation, checking behavior, and OCD $J$ Behav Ther Exp Psychiatry 49:133-140.

Hinds AL, Woody EZ, Van Ameringen M, Schmidt LA, and Szechtman H (2012) When too much is not enough: obsessive-compulsive disorder as a pathology of stopping, rather than starting. PLoS One 7:e30586.

Ho EV, Thompson SL, Katzka WR, Sharifi MF, Knowles JA, and Dulawa SC (2016) Clinically effective OCD treatment prevents 5-HT1B receptor-induced repetitive behavior and striatal activation. Psychopharmacology (Berl) 233:57-70

Hoehn-Saric R, Ninan P, Black DW, Stahl S, Greist JH, Lydiard B, McElroy S, Zajecka J, Chapman D, Clary C, et al. (2000) Multicenter double-blind comparison of sertraline and desipramine for concurrent obsessive-compulsive and major depressive disorders. Arch Gen Psychiatry 57:76-82.

Hoffman KL, Hornig M, Yaddanapudi K, Jabado O, and Lipkin WI (2004) A murine model for neuropsychiatric disorders associated with group A beta-hemolytic streptococcal infection. $J$ Neurosci 24:1780-1791.

Hoffman KL and Rueda Morales RI (2009) Toward an understanding of the neurobiology of "just right" perceptions: nest building in the female rabbit as a possible model for compulsive behavior and the perception of task completion. Behav Brain Res 204:182-191.
Hoffman KL and Rueda Morales RI (2012) D1 and D2 dopamine receptor antagonists decrease behavioral bout duration, without altering the bout's repeated behavioral components, in a naturalistic model of repetitive and compulsive behavior. Behav Brain Res 230:1-10.

Hofmann SG, Meuret AE, Smits JA, Simon NM, Pollack MH, Eisenmenger K, Shiekh M, and Otto MW (2006) Augmentation of exposure therapy with D-cycloserine for social anxiety disorder. Arch Gen Psychiatry 63:298-304.

Hollander E, DeCaria CM, Nitescu A, Gully R, Suckow RF, Cooper TB, Gorman JM, Klein DF, and Liebowitz MR (1992) Serotonergic function in obsessive-compulsive disorder. Behavioral and neuroendocrine responses to oral m-chlorophenylpiperazine and fenfluramine in patients and healthy volunteers. Arch Gen Psychiatry 49:21-28.

Hollander E, Kaplan A, Allen A, and Cartwright C (2000) Pharmacotherapy for obsessive-compulsive disorder. Psychiatr Clin North Am 23:643-656.

Holmseth S, Dehnes Y, Huang YH, Follin-Arbelet VV, Grutle NJ, Mylonakou MN, Plachez C, Zhou Y, Furness DN, Bergles DE, et al. (2012) The density of EAAC1 (EAAT3) glutamate transporters expressed by neurons in the mammalian CNS. $J$ Neurosci 32:6000-6013.

Honma K and Honma S (2009) The SCN-independent clocks, methamphetamine and food restriction. Eur $J$ Neurosci 30:1707-1717.

House SJ, Tripathi SP, Knight BT, Morris N, Newport DJ, and Stowe ZN (2016) Obsessive-compulsive disorder in pregnancy and the postpartum period: course of illness and obstetrical outcome. Arch Women Ment Health 19:3-10.

Houslay MD and Milligan G (1997) Tailoring cAMP-signalling responses through isoform multiplicity. Trends Biochem Sci 22:217-224.

Houslay MD, Sullivan M, and Bolger GB (1998) The multienzyme PDE4 cyclic adenosine monophosphate-specific phosphodiesterase family: intracellular targeting, regulation, and selective inhibition by compounds exerting anti-inflammatory and antidepressant actions. Adv Pharmacol 44:225-342.

Huey ED, Zahn R, Krueger F, Moll J, Kapogiannis D, Wassermann EM, and Grafman J (2008) A psychological and neuroanatomical model of obsessive-compulsive disorder. $J$ Neuropsychiatry Clin Neurosci 20:390-408.

Humble MB, Uvnäs-Moberg K, Engström I, and Bejerot S (2013) Plasma oxytocin changes and anti-obsessive response during serotonin reuptake inhibitor treatment: a placebo controlled study. BMC Psychiatry 13:344.

Hyman SE (2010) The diagnosis of mental disorders: the problem of reification. Annu Rev Clin Psychol 6:155-179.

Idro R, Maling S, and Byarugaba J (2005) Compulsive behavior and coprolalia after cerebral malaria. J Pediatr Neurol 3:107-108.

Insel TR (1992) Toward a neuroanatomy of obsessive-compulsive disorder. Arch Gen Psychiatry 49:739-744.

Issari Y, Jakubovski E, Bartley CA, Pittenger C, and Bloch MH (2016) Early onset of response with selective serotonin reuptake inhibitors in obsessive-compulsive disorder: a meta-analysis. J Clin Psychiatry 77:e605-e611.

Iyo M, Bi Y, Hashimoto K, Inada T, and Fukui S (1996) Prevention of methamphetamine-induced behavioral sensitization in rats by a cyclic AMP phosphodiesterase inhibitor, rolipram. Eur J Pharmacol 312:163-170.

Jacobs BL and Azmitia EC (1992) Structure and function of the brain serotonin system. Physiol Rev 72:165-229.

Jacobs BL and Fornal CA (1995) Serotonin and behavior: a general hypothesis, in Psychopharmacology: The Fourth Generation of Progress (Bloom FE and Kupfer DJ eds) pp 461-470, Raven Press, New York.

Jaspers K (1963) General Psychopathology (Translated from the German by J. Hoenig and Marian W. Hamilton), University of Chicago Press, Chicago.

Jaworska-Feil L, Budziszewska B, Leśkiewicz M, and Lasoń W (1998) Opposite effects of inhibitory and excitatory neurosteroids on $[3 \mathrm{H}]$ dopamine release from rat nucleus accumbens. Pol J Pharmacol 50:449-452.

Jenike MA (1983) Obsessive compulsive disorder. Compr Psychiatry 24:99-115.

Jenike MA, Baer L, Ballantine T, Martuza RL, Tynes S, Giriunas I, Buttolph ML, and Cassem NH (1991) Cingulotomy for refractory obsessive-compulsive disorder. A long-term follow-up of 33 patients. Arch Gen Psychiatry 48:548-555.

Joel D (2006) The signal attenuation rat model of obsessive-compulsive disorder: a review. Psychopharmacology (Berl) 186:487-503.

Joel D and Doljansky J (2003) Selective alleviation of compulsive lever-pressing in rats by $\mathrm{D} 1$, but not $\mathrm{D} 2$, blockade: possible implications for the involvement of D1 receptors in obsessive-compulsive disorder. Neuropsychopharmacology 28:77-85.

Johnson EF and Szechtman H (2016) A dose-response study of separate and combined effects of the serotonin agonist 8-OH-DPAT and the dopamine agonist quinpirole on locomotor sensitization, cross-sensitization, and conditioned activity. Behav Phar macol 27:439-450.

Johnson JC, Scott JG, and Jess N (2016) Assessing the effectiveness of pharmacotherapy for obsessive-compulsive disorder. Lancet Psychiatry 3:e16.

Jonsson H, Kristensen M, and Arendt M (2015) Intensive cognitive behavioural therapy for obsessive-compulsive disorder: a systematic review and meta-analysis. JOCRD 6:83-96.

Jung TD, Jung PS, Raveendran L, Farbod Y, Dvorkin-Gheva A, Sakic B, Surette MG, and Szechtman $\mathrm{H}$ (2018) Changes in gut microbiota during development of compulsive checking and locomotor sensitization induced by chronic treatment with the dopamine agonist quinpirole. Behav Pharmacol 29:211-224.

Jury NJ, McCormick BA, Horseman ND, Benoit SC, and Gregerson KA (2015) Enhanced responsiveness to selective serotonin reuptake inhibitors during lactation. PLoS One 10:e117339.

Kalsbeek A, Yi CX, Cailotto C, la Fleur SE, Fliers E, and Buijs RM (2011) Mammalian clock output mechanisms. Essays Biochem 49:137-151.

Kanai Y, Clémencon B, Simonin A, Leuenberger M, Lochner M, Weisstanner M, and Hediger MA (2013) The SLC1 high-affinity glutamate and neutral amino acid transporter family. Mol Aspects Med 34:108-120.

Kantak PA, Bobrow DN, and Nyby JG (2014) Obsessive-compulsive-like behaviors in house mice are attenuated by a probiotic (Lactobacillus rhamnosus GG). Behav Pharmacol 25:71-79.

Kapadia M and Sakic B (2011) Autoimmune and inflammatory mechanisms of CNS damage. Prog Neurobiol 95:301-333. 
Kapur S and Remington G (1996) Serotonin-dopamine interaction and its relevance to schizophrenia. Am J Psychiatry 153:466-476.

Katerberg H, Lochner C, Cath DC, de Jonge P, Bochdanovits Z, Moolman-Smook JC, Hemmings SM, Carey PD, Stein DJ, Sondervan D, et al. (2009) The role of the brainderived neurotrophic factor (BDNF) val66met variant in the phenotypic expression of obsessive-compulsive disorder (OCD). Am J Med Genet B Neuropsychiatr Genet 150B:1050-1062.

Katzman MA, Bleau P, Blier P, Chokka P, Kjernisted K, Van Ameringen M, Antony MM, Bouchard S, Brunet A, Flament M, et al.; Canadian Anxiety Guidelines Initiative Group on behalf of the Anxiety Disorders Association of Canada/Association Canadienne des troubles anxieux and McGill University (2014) Canadian clinical practice guidelines for the management of anxiety, posttraumatic stress and obsessive-compulsive disorders. BMC Psychiatry 14 (Suppl 1):S1.

Katzung BG (2017) Basic and Clinical Pharmacology, McGraw-Hill Education, New York.

Kelmendi B, Holsbach-Beltrame M, McIntosh AM, Hilt L, George ED, Kitchen RR Carlyle BC, Pittenger C, Coric V, Nolen-Hoeksema S, et al. (2011) Association of polymorphisms in HCN4 with mood disorders and obsessive compulsive disorder. Neurosci Lett 496:195-199.

Kendler KS (2008) Explanatory models for psychiatric illness. Am J Psychiatry 165: 695-702

Kettenmann H, Hanisch UK, Noda M, and Verkhratsky A (2011) Physiology of microglia. Physiol Rev 91:461-553.

Kettenmann H, Kirchhoff F, and Verkhratsky A (2013) Microglia: new roles for the synaptic stripper. Neuron 77:10-18.

Kettl PA and Marks IM (1986) Neurological factors in obsessive compulsive disorder. Two case reports and a review of the literature. Br J Psychiatry 149:315-319.

Khanna S, John JP, and Reddy LP (2001) Neuroendocrine and behavioral responses to mCPP in obsessive-compulsive disorder. Psychoneuroendocrinology 26:209-223.

Kiesler DJ (1996) Contemporary Interpersonal Theory and Research: Personality, Psychopathology, and Psychotherapy, Wiley, New York.

Kim CH, Koo MS, Cheon KA, Ryu YH, Lee JD, and Lee HS (2003) Dopamine transporter density of basal ganglia assessed with [123I]IPT SPET in obsessivecompulsive disorder. Eur J Nucl Med Mol Imaging 30:1637-1643.

Kim H, McGrath BM, and Silverstone PH (2005) A review of the possible relevance of inositol and the phosphatidylinositol second messenger system (PI-cycle) to psychiatric disorders--focus on magnetic resonance spectroscopy (MRS) studies. Hum Psychopharmacol 20:309-326.

Kim JJ, Lee MC, Kim J, Kim IY, Kim SI, Han MH, Chang KH, and Kwon JS (2001) Grey matter abnormalities in obsessive-compulsive disorder: statistical parametric mapping of segmented magnetic resonance images. $\mathrm{Br}$ J Psychiatry 179:330-334.

Kim SW, Dysken MW, and Kuskowski M (1990) The Yale-Brown ObsessiveCompulsive Scale: a reliability and validity study. Psychiatry Res 34:99-106.

Kirsch I (2005) Placebo psychotherapy: synonym or oxymoron? J Clin Psychol 61: $791-803$

Kisely S, Hall K, Siskind D, Frater J, Olson S, and Crompton D (2014) Deep brain stimulation for obsessive-compulsive disorder: a systematic review and metaanalysis. Psychol Med 44:3533-3542.

Klavir O, Flash S, Winter C, and Joel D (2009) High frequency stimulation and pharmacological inactivation of the subthalamic nucleus reduces 'compulsive' leverpressing in rats. Exp Neurol 215:101-109.

Klavir O, Winter C, and Joel D (2011) High but not low frequency stimulation of both the globus pallidus and the entopeduncular nucleus reduces 'compulsive' leverpressing in rats. Behav Brain Res 216:84-93.

Kluge M, Schüssler P, Künzel HE, Dresler M, Yassouridis A, and Steiger A (2007) Increased nocturnal secretion of ACTH and cortisol in obsessive compulsive disorder. J Psychiatr Res 41:928-933.

Koh MJ, Seol J, Kang JI, Kim BS, Namkoong K, Chang JW, and Kim SJ (2018) Altered resting-state functional connectivity in patients with obsessive-compulsive disorder: a magnetoencephalography study. Int J Psychophysiol 123:80-87.

Kontis D, Tsaltas E, Boulougouris V, Papakosta VM, Kalogerakou S, Papadopoulos S, and Papadimitriou G (2008) 5-HT2A and 5-HT2C receptor involvement in the acute effects of mCPP and fluoxetine on persistence behaviour. Int $J$ Neuropsychopharmacol 11:282.

Konturek PC, Brzozowski T, and Konturek S.J (2011) Gut clock: implication of circadian rhythms in the gastrointestinal tract. J Physiol Pharmacol 62:139-150.

Konuk N, Tekin IO, Ozturk U, Atik L, Atasoy N, Bektas S, and Erdogan A (2007) Plasma levels of tumor necrosis factor-alpha and interleukin-6 in obsessive compulsive disorder. Mediators Inflamm 2007:65704.

Koran LM, Hanna GL, Hollander E, Nestadt G, and Simpson HB; American Psychiatric Association (2007) Practice guideline for the treatment of patients with obsessive-compulsive disorder. Am J Psychiatry 164 ((Suppl):5-53.

Koran LM and Simpson HB (2013) Guideline Watch (2013): Practice Guideline for the Treatment of Patients with Obsessive-Compulsive Disorder, American Psychiatric Association, Arlington, VA.

Korff S, Stein DJ, and Harvey BH (2008) Stereotypic behaviour in the deer mouse: pharmacological validation and relevance for obsessive compulsive disorder. Prog Neuropsychopharmacol Biol Psychiatry 32:348-355.

Korff S, Stein DJ, and Harvey BH (2009) Cortico-striatal cyclic AMP-phosphodiesterase-4 signalling and stereotypy in the deer mouse: attenuation after chronic fluoxetine treatment. Pharmacol Biochem Behav 92:514-520.

Krasnova IN, Justinova Z, and Cadet JL (2016) Methamphetamine addiction: involvement of CREB and neuroinflammatory signaling pathways. Psychopharmacology (Berl) 233:1945-1962.

Krass M, Rünkorg K, Wegener G, and Volke V (2010) Nitric oxide is involved in the regulation of marble-burying behavior. Neurosci Lett 480:55-58.

Kraus C, Castrén E, Kasper S, and Lanzenberger R (2017) Serotonin and neuroplasticity - links between molecular, functional and structural pathophysiology in depression. Neurosci Biobehav Rev 77:317-326.
Kuloglu M, Atmaca M, Tezcan E, Gecici O, Tunckol H, and Ustundag B (2002) Antioxidant enzyme activities and malondialdehyde levels in patients with obsessivecompulsive disorder. Neuropsychobiology 46:27-32.

Kumar A, Williams MT, and Chugani HT (2015) Evaluation of basal ganglia and thalamic inflammation in children with pediatric autoimmune neuropsychiatric disorders associated with streptococcal infection and Tourette syndrome: a positron emission tomographic (PET) study using 11C-[R]-PK11195. J Child Neurol 30:749-756.

Lange KW, Lange KM, Hauser J, Tucha L, and Tucha O (2012) Circadian rhythms in obsessive-compulsive disorder. J Neural Transm (Vienna) 119:1077-1083.

Laplane D, Levasseur M, Pillon B, Dubois B, Baulac M, Mazoyer B, Tran Dinh S, Sette G, Danze F, and Baron JC (1989) Obsessive-compulsive and other behavioural changes with bilateral basal ganglia lesions. A neuropsychological, magnetic resonance imaging and positron tomography study. Brain 112:699-725.

Laube G and Bernstein HG (2017) Agmatine: multifunctional arginine metabolite and magic bullet in clinical neuroscience? Biochem $J$ 474:2619-2640.

Leckman JF, Denys D, Simpson HB, Mataix-Cols D, Hollander E, Saxena S, Miguel EC, Rauch SL, Goodman WK, Phillips KA, et al. (2010) Obsessive-compulsive disorder: a review of the diagnostic criteria and possible subtypes and dimensional specifiers for DSM-V. Depress Anxiety 27:507-527.

Leckman JF, Goodman WK, North WG, Chappell PB, Price LH, Pauls DL, Anderson GM, Riddle MA, McSwiggan-Hardin M, McDougle CJ, et al. (1994) Elevated cerebrospinal fluid levels of oxytocin in obsessive-compulsive disorder. Comparison with Tourette's syndrome and healthy controls. Arch Gen Psychiatry 51:782-792.

Leckman JF, Mayes LC, Feldman R, Evans DW, King RA, and Cohen DJ (1999) Early parental preoccupations and behaviors and their possible relationship to the symptoms of obsessive-compulsive disorder. Acta Psychiatr Scand Suppl 396:1-26.

Leckman JF, et al. (2005) Increased serum levels of interleukin-12 and tumor necrosis factor-alpha in Tourette's syndrome. Biol Psychiatry 57 (6):667-673, doi: 10.1016/ j.biopsych.2004.12.004 15780855

Lefaucheur JP, André-Obadia N, Antal A, Ayache SS, Baeken C, Benninger DH Cantello RM, Cincotta M, de Carvalho M, De Ridder D, et al. (2014) Evidence-based guidelines on the therapeutic use of repetitive transcranial magnetic stimulation (rTMS). Clin Neurophysiol 125:2150-2206.

Lefaucheur JP, Antal A, Ayache SS, Benninger DH, Brunelin J, Cogiamanian F, Cotelli M, De Ridder D, Ferrucci R, Langguth B, et al. (2017) Evidence-based guidelines on the therapeutic use of transcranial direct current stimulation (tDCS). Clin Neurophysiol 128:56-92.

Lehmann ML, Cooper HA, Maric D, and Herkenham M (2016) Social defeat induces depressive-like states and microglial activation without involvement of peripheral macrophages. J Neuroinflammation 13:224.

Leiphart JW and Valone FH 3rd (2010) Stereotactic lesions for the treatment of psychiatric disorders. $J$ Neurosurg 113:1204-1211.

Leonard HL, Swedo SE, Rapoport JL, Koby EV, Lenane MC, Cheslow DL, and Hamburger SD (1989) Treatment of obsessive-compulsive disorder with clomipramine and desipramine in children and adolescents. A double-blind crossover comparison. Arch Gen Psychiatry 46:1088-1092.

Lesch KP and Waider J (2012) Serotonin in the modulation of neural plasticity and networks: implications for neurodevelopmental disorders. Neuron $\mathbf{7 6}$ $175-191$

Leung JG and Palmer BA (2016) Psychosis or obsessions? Clozapine associated with worsening obsessive-compulsive symptoms. Case Rep Psychiatry 2016:2180748.

Levine J, Mishori A, Susnosky M, Martin M, and Belmaker RH (1999) Combination of inositol and serotonin reuptake inhibitors in the treatment of depression. Biol Psychiatry 45:270-273

Levy R and Meyer V (1971) Ritual prevention in obsessional patients. Proc R Soc Med 64: $1115-1118$

Lewin AB, Wu MS, McGuire JF, and Storch EA (2014) Cognitive behavior therapy for obsessive-compulsive and related disorders. Psychiatr Clin North Am 37:415-445.

Li H, Zuo J, and Tang W (2018) Phosphodiesterase-4 inhibitors for the treatment of inflammatory diseases. Front Pharmacol 9:1048.

Lienard P (2011) Life stages and risk-avoidance: status- and context-sensitivity in precaution systems. Neurosci Biobehav Rev 35:1067-1074.

Lissemore JI, Sookman D, Gravel P, Berney A, Barsoum A, Diksic M, Nordahl TE Pinard G, Sibon I, Cottraux J, et al. (2018) Brain serotonin synthesis capacity in obsessive-compulsive disorder: effects of cognitive behavioral therapy and sertraline. Transl Psychiatry 8:82.

Lochner C, Seedat S, du Toit PL, Nel DG, Niehaus DJ, Sandler R, and Stein DJ (2005) Obsessive-compulsive disorder and trichotillomania: a phenomenological comparison. BMC Psychiatry 5:2.

Logan RW and Sarkar DK (2012) Circadian nature of immune function. Mol Cell Endocrinol 349:82-90.

Loiselle CR, Lee O, Moran TH, and Singer HS (2004) Striatal microinfusion of Tourette syndrome and PANDAS sera: failure to induce behavioral changes. Mov Disord 19:390-396.

Lopes AC, Greenberg BD, Canteras MM, Batistuzzo MC, Hoexter MQ, Gentil AF, Pereira CA, Joaquim MA, de Mathis ME, D'Alcante CC, et al. (2014) Gamma ventral capsulotomy for obsessive-compulsive disorder: a randomized clinical trial. JAMA Psychiatry 71:1066-1076.

López-Ibor JJ Jr, Saiz J, Cottraux J, Note I, Viñas R, Bourgeois M, Hernández M, and Gómez-Pérez JC (1996) Double-blind comparison of fluoxetine versus clomipramine in the treatment of obsessive compulsive disorder. Eur Neuropsychopharmacol 6:111-118.

Lopresti AL (2017) Curcumin for neuropsychiatric disorders: a review of in vitro, animal and human studies. $J$ Psychopharmacol 31:287-302.

Lorrain DS, Riolo JV, Matuszewich L, and Hull EM (1999) Lateral hypothalamic serotonin inhibits nucleus accumbens dopamine: implications for sexual satiety. $J$ Neurosci 19:7648-7652.

Lotan D, Benhar I, Alvarez K, Mascaro-Blanco A, Brimberg L, Frenkel D, Cunningham MW, and Joel D (2014) Behavioral and neural effects of intra-striatal infusion of anti-streptococcal antibodies in rats. Brain Behav Immun 38:249-262. 
Lutz TA (2011) Steve Woods's contribution to research on amylin's eating inhibitory effect. Physiol Behav 103:25-30.

Luyten L, Hendrickx S, Raymaekers S, Gabriëls L, and Nuttin B (2016) Electrical stimulation in the bed nucleus of the stria terminalis alleviates severe obsessivecompulsive disorder. Mol Psychiatry 21:1272-1280.

MacIsaac SE, Carvalho AF, Cha DS, Mansur RB, and McIntyre RS (2014) The mechanism, efficacy, and tolerability profile of agomelatine. Expert Opin Pharmacother 15:259-274.

MacLean PD (1978) Effects of lesions of globus pallidus on species-typical display behavior of squirrel monkeys. Brain Res 149:175-196.

Macrì S, Ceci C, Onori MP, Invernizzi RW, Bartolini E, Altabella L, Canese R, Imperi M, Orefici G, Creti R, et al. (2015) Mice repeatedly exposed to group-A $\beta$-haemolytic Streptococcus show perseverative behaviors, impaired sensorimotor gating, and immune activation in rostral diencephalon. Sci Rep 5:13257.

Macrì S, Spinello C, Widomska J, Magliozzi R, Poelmans G, Invernizzi RW, Creti R, Roessner V, Bartolini E, Margarit I, et al. (2018) Neonatal corticosterone mitigates autoimmune neuropsychiatric disorders associated with Streptococcus in mice. Sci Rep 8:10188.

Maguire EA, Woollett K, and Spiers HJ (2006) London taxi drivers and bus drivers: a structural MRI and neuropsychological analysis. Hippocampus 16:1091-1101.

Mallet L, Mesnage V, Houeto JL, Pelissolo A, Yelnik J, Behar C, Gargiulo M, Welter ML, Bonnet AM, Pillon B, et al. (2002) Compulsions, Parkinson's disease, and stimulation. Lancet 360:1302-1304.

Mallet L, Polosan M, Jaafari N, Baup N, Welter ML, Fontaine D, du Montcel ST, Yelnik J, Chéreau I, Arbus C, et al.; STOC Study Group (2008) Subthalamic nucleus stimulation in severe obsessive-compulsive disorder. N Engl J Med 359:2121-2134.

Mantione M, Nieman DH, Figee M, and Denys D (2014) Cognitive-behavioural therapy augments the effects of deep brain stimulation in obsessive-compulsive disorder. Psychol Med 44:3515-3522.

Marazziti D, Baroni S, Palego L, Masala I, Consoli G, Catena Dell'osso M, Giannaccini G, and Lucacchini A (2009) Adenylate-cyclase activity in platelets of patients with obsessive-compulsive disorder. Neuropsychiatr Dis Treat 5:363-367.

Marazziti D, Dell'Osso L, Masala I, Baroni S, Presta S, Giannaccini G, Di Nasso E, Mungai F, Lucacchini A, and Cassano GB (2002) Decreased inhibitory activity of $\mathrm{PKC}$ in OCD patients after six months of treatment. Psychoneuroendocrinology 27: 769-776.

Marazziti D, Masala I, Rossi A, Hollander E, Presta S, Giannaccini G, Mazzoni MR, Dell'Osso L, Lucacchini A, and Cassano GB (2000) Increased inhibitory activity of protein kinase $\mathrm{C}$ on the serotonin transporter in OCD. Neuropsychobiology 41: 171-177.

Marazziti D, Perez J, and Cassano GB (2001) Is obsessive-compulsive disorder caused by a second-messenger imbalance? CNS Spectr 6:206-209.

Markarian Y, Larson MJ, Aldea MA, Baldwin SA, Good D, Berkeljon A, Murphy TK Storch EA, and McKay D (2010) Multiple pathways to functional impairment in obsessive-compulsive disorder. Clin Psychol Rev 30:78-88.

Marks IM and Nesse RM (1994) Fear and fitness: an evolutionary analysis of anxiety disorders. Ethol Sociobiol 15:247-261.

Masi G, Millepiedi S, Perugi G, Pfanner C, Berloffa S, Pari C, Mucci M, and Akiskal HS (2010) A naturalistic exploratory study of the impact of demographic, phenotypic and comorbid features in pediatric obsessive-compulsive disorder. Psychopathology 43:69-78.

Mason JW (1968) A review of psychoendocrine research on the pituitary-adrenal cortical system. Psychosom Med 30:576-607.

Masterson FA and Crawford M (1982) The defense motivation system - a theory of avoidance-behavior. Behav Brain Sci 5:661-675.

Mataix-Cols D, Fernández de la Cruz L, Monzani B, Rosenfield D, Andersson E, PérezVigil A, Frumento P, de Kleine RA, Difede J, Dunlop BW, et al.; DCS Anxiety Consortium (2017) D-cycloserine augmentation of exposure-based cognitive behavior therapy for anxiety, obsessive-compulsive, and posttraumatic stress disorders: a systematic review and meta-analysis of individual participant data. JAMA Psychiatry 74:501-510.

Mataix-Cols D, Fernández de la Cruz L, Nordsletten AE, Lenhard F, Isomura K, and Simpson HB (2016) Towards an international expert consensus for defining treatment response, remission, recovery and relapse in obsessive-compulsive disorder. World Psychiatry 15:80-81.

Mataix-Cols D, Rosario-Campos MC, and Leckman JF (2005) A multidimensional model of obsessive-compulsive disorder. Am J Psychiatry 162:228-238.

Mataix-Cols D, Wooderson S, Lawrence N, Brammer MJ, Speckens A, and Phillips ML (2004) Distinct neural correlates of washing, checking, and hoarding symptom dimensions in obsessive-compulsive disorder. Arch Gen Psychiatry 61:564-576.

Mathews GC and Diamond JS (2003) Neuronal glutamate uptake contributes to GABA synthesis and inhibitory synaptic strength. J Neurosci 23:2040-2048.

Mathis MA, de Alvarenga P, Funaro G, Torresan RC, Moraes I, Torres AR, Zilberman ML, and Hounie AG (2011) Gender differences in obsessive-compulsive disorder: a literature review. Br J Psychiatry 33:390-399.

Matsumoto R, Ichise M, Ito H, Ando T, Takahashi H, Ikoma Y, Kosaka J, Arakawa R, Fujimura Y, Ota M, et al. (2010) Reduced serotonin transporter binding in the insular cortex in patients with obsessive-compulsive disorder: a [11C]DASB PET study. Neuroimage 49:121-126.

Matsunaga H, Hayashida K, Kiriike N, Maebayashi K, and Stein DJ (2010) The clinical utility of symptom dimensions in obsessive-compulsive disorder. Psychiatry Res 180:25-29.

Matsushita M, Egashira N, Harada S, Okuno R, Mishima K, Iwasaki K, Nishimura R, and Fujiwara M (2005) Perospirone, a novel antipsychotic drug, inhibits marbleburying behavior via 5-HT1A receptor in mice: implications for obsessive-compulsive disorder. J Pharmacol Sci 99:154-159.

Mattson MP, Maudsley S, and Martin B (2004) BDNF and 5-HT: a dynamic duo in age-related neuronal plasticity and neurodegenerative disorders. Trends Neurosci 27:589-594.
Mavridis M, Colpaert FC, and Millan MJ (1991) Differential modulation of (+)-amphetamine-induced rotation in unilateral substantia nigra-lesioned rats by alpha 1 as compared to alpha 2 agonists and antagonists. Brain Res 562:216-224.

Maya Vetencourt JF, Sale A, Viegi A, Baroncelli L, De Pasquale R, O'Leary OF, Castrén E, and Maffei L (2008) The antidepressant fluoxetine restores plasticity in the adult visual cortex. Science 320:385-388.

Mazzio EA, Harris N, and Soliman KF (1998) Food constituents attenuate monoamine oxidase activity and peroxide levels in $\mathrm{C} 6$ astrocyte cells. Planta Med 64: 603-606.

McDougle CJ, Barr LC, Goodman WK, and Price LH (1999) Possible role of neuropeptides in obsessive compulsive disorder. Psychoneuroendocrinology 24:1-24.

McDougle CJ, Goodman WK, Price LH, Delgado PL, Krystal JH, Charney DS, and Heninger GR (1990) Neuroleptic addition in fluvoxamine-refractory obsessivecompulsive disorder. Am J Psychiatry 147:652-654.

McGrath MJ, Campbell KM, Parks CR, and Burton FH (2000) Glutamatergic drugs exacerbate symptomatic behavior in a transgenic model of comorbid Tourette's syndrome and obsessive-compulsive disorder. Brain Res 877:23-30.

McGuinness M, Blissett J, and Jones C (2011) OCD in the perinatal period: is postpartum OCD (ppOCD) a distinct subtype? A review of the literature. Behav Cogn Psychother 39:285-310.

McGuire JF, Lewin AB, and Storch EA (2014) Enhancing exposure therapy for anxiety disorders, obsessive-compulsive disorder and post-traumatic stress disorder. Expert Rev Neurother 14:893-910.

McGuire JF, Wu MS, Piacentini J, McCracken JT, and Storch EA (2017) A metaanalysis of d-cycloserine in exposure-based treatment: moderators of treatment efficacy, response, and diagnostic remission. J Clin Psychiatry 78:196-206.

McGuire PK, Bench CJ, Frith CD, Marks IM, Frackowiak RS, and Dolan RJ (1994) Functional anatomy of obsessive-compulsive phenomena. $\mathrm{Br} J$ Psychiatry 164: $459-468$.

McIntyre CC and Hahn PJ (2010) Network perspectives on the mechanisms of deep brain stimulation. Neurobiol Dis 38:329-337.

McKay D, Sookman D, Neziroglu F, Wilhelm S, Stein DJ, Kyrios M, Matthews K, and Veale D; Accreditation Task Force of The Canadian Institute for Obsessive Compulsive Disorders (2015) Efficacy of cognitive-behavioral therapy for obsessivecompulsive disorder. Psychiatry Res 227:104-113.

McLean PD, Whittal ML, Thordarson DS, Taylor S, Söchting I, Koch WJ, Paterson R, and Anderson KW (2001) Cognitive versus behavior therapy in the group treatment of obsessive-compulsive disorder. J Consult Clin Psychol 69:205-214.

Medzhitov R, Schneider DS, and Soares MP (2012) Disease tolerance as a defense strategy. Science 335:936-941.

Menzies L, Chamberlain SR, Laird AR, Thelen SM, Sahakian BJ, and Bullmore ET (2008) Integrating evidence from neuroimaging and neuropsychological studies of obsessive-compulsive disorder: the orbitofronto-striatal model revisited. Neurosci Biobehav Rev 32:525-549.

Mercier H and Sperber D (2017) The Enigma of Reason, Harvard University Press, Cambridge, MA.

Messaoudi M, Violle N, Bisson JF, Desor D, Javelot H, and Rougeot C (2011) Beneficial psychological effects of a probiotic formulation (Lactobacillus helveticus R0052 and Bifidobacterium longum R0175) in healthy human volunteers. Gut Microbes 2: 256-261.

Mestre TA, Lang AE, and Okun MS (2016) Factors influencing the outcome of deep brain stimulation: placebo, nocebo, lessebo, and lesion effects. Mov Disord 31: 290-296.

Meyer V (1966) Modification of expectations in cases with obsessional rituals. Behav Res Ther 4:273-280.

Milad MR and Rauch SL (2012) Obsessive-compulsive disorder: beyond segregated cortico-striatal pathways. Trends Cogn Sci 16:43-51.

Miller AH, Maletic V, and Raison CL (2009) Inflammation and its discontents: the role of cytokines in the pathophysiology of major depression. Biol Psychiatry 65:732-741.

Miman O, Mutlu EA, Ozcan O, Atambay M, Karlidag R, and Unal S (2010) Is there any role of Toxoplasma gondii in the etiology of obsessive-compulsive disorder? Psychiatry Res 177:263-265.

Mistlberger RE and Antle MC (2011) Entrainment of circadian clocks in mammals by arousal and food. Essays Biochem 49:119-136.

Mistlberger RE, Antle MC, Glass JD, and Miller JD (2000) Behavioral and serotonergic regulation of circadian rhythms. Biol Rhythm Res 31:240-283.

Mitra S, Bastos CP, Bates K, Pereira GS, and Bult-Ito A (2016) Ovarian sex hormones modulate compulsive, affective and cognitive functions in a non-induced mouse model of obsessive-compulsive disorder. Front Behav Neurosci 10:215.

Mitra S, Bastos CP, Chesworth S, Frye C, and Bult-Ito A (2017) Strain and sex based characterization of behavioral expressions in non-induced compulsive-like mice. Physiol Behav 168:103-111.

Mittleman BB, Castellanos FX, Jacobsen LK, Rapoport JL, Swedo SE, and Shearer GM (1997) Cerebrospinal fluid cytokines in pediatric neuropsychiatric disease. J Immunol 159:2994-2999.

Modarresi A, Sayyah M, Razooghi S, Eslami K, Javadi M, and Kouti L (2018) Memantine augmentation improves symptoms in serotonin reuptake inhibitor-refractory obsessive-compulsive disorder: a randomized controlled trial. Pharmacopsychiatry 51:263-269.

Modell JG, Mountz JM, Curtis GC, and Greden JF (1989) Neurophysiologic dysfunction in basal ganglia/limbic striatal and thalamocortical circuits as a pathogenetic mechanism of obsessive-compulsive disorder. J Neuropsychiatry Clin Neurosci 1:27-36.

Möller M, Swanepoel T, and Harvey BH (2015) Neurodevelopmental animal models reveal the convergent role of neurotransmitter systems, inflammation, and oxidative stress as biomarkers of schizophrenia: implications for novel drug development. ACS Chem Neurosci 6:987-1016.

Monteiro P and Feng G (2016) Learning from animal models of obsessive-compulsive disorder. Biol Psychiatry 79:7-16. 
Monteleone P, Catapano F, Tortorella A, and Maj M (1997) Cortisol response to d-fenfluramine in patients with obsessive-compulsive disorder and in healthy subjects: evidence for a gender-related effect. Neuropsychobiology 36:8-12.

Montgomery EB Jr (2007) Basal ganglia physiology and pathophysiology: a reappraisal. Parkinsonism Relat Disord 13:455-465.

Montgomery EB Jr (2012) The epistemology of deep brain stimulation and neuronal pathophysiology. Front Integr Nuerosci 6:78.

Montgomery EB Jr (2016) Modeling and theories of pathophysiology and physiology of the basal ganglia-thalamic-cortical system: critical analysis. Front Hum Neurosci 10:469.

Montgomery EB Jr (2017) Deep Brain Stimulation Programming: Mechanisms, Principles, And Practice (Kindle Edition), Oxford University Press, New York.

Montgomery EB Jr and Gale JT (2008) Mechanisms of action of deep brain stimulation (DBS). Neurosci Biobehav Rev 32:388-407.

Montgomery EB Jr and He H (2016) Deep brain stimulation frequency-a divining rod for new and novel concepts of nervous system function and therapy. Brain Sci 6:E34.

Moran TH (2004) Gut peptides in the control of food intake: 30 years of ideas. Physiol Behav 82:175-180.

Moriana JA, Gálvez-Lara M, and Corpas J (2017) Psychological treatments for mental disorders in adults: a review of the evidence of leading international organizations. Clin Psychol Rev 54:29-43.

Morris CJ, Aeschbach D, and Scheer FA (2012) Circadian system, sleep and endocrinology. Mol Cell Endocrinol 349:91-104.

Mrosovsky N (1996) Locomotor activity and non-photic influences on circadian clocks. Biol Rev Camb Philos Soc 71:343-372.

Murphy TK, Gerardi DM, and Leckman JF (2014) Pediatric acute-onset neuropsychiatric syndrome. Psychiatr Clin North Am 37:353-374.

Murphy TK, Mutch PJ, Reid JM, Edge PJ, Storch EA, Bengtson M, and Yang M (2009) Open label aripiprazole in the treatment of youth with tic disorders. J Child Adolesc Psychopharmacol 19:441-447.

Nakao T, Okada K, and Kanba S (2014) Neurobiological model of obsessive-compulsive disorder: evidence from recent neuropsychological and neuroimaging findings. Psychiatry Clin Neurosci 68:587-605.

National Collaborating Centre for Mental Health (2006) Obsessive-Compulsive Disorder: Core Interventions in the Treatment of Obsessive-Compulsive Disorder and Body Dysmorphic Disorder, British Psychological Society and Royal College of Psychiatrists, Leicester, UK.

Neis VB, Rosa PB, Olescowicz G, and Rodrigues ALS (2017) Therapeutic potential of agmatine for CNS disorders. Neurochem Int 108:318-331.

Nestler EJ and Hyman SE (2010) Animal models of neuropsychiatric disorders. Nat Neurosci 13:1161-1169.

Neufeld KA, Kang N, Bienenstock J, and Foster JA (2011a) Effects of intestinal microbiota on anxiety-like behavior. Commun Integr Biol 4:492-494.

Neufeld KM, Kang N, Bienenstock J, and Foster JA (2011b) Reduced anxiety-like behavior and central neurochemical change in germ-free mice. Neurogastroenterol Motil 23:255-264, e119.

Neumaier F, Paterno M, Alpdogan S, Tevoufouet EE, Schneider T, Hescheler J, and Albanna W (2017) Surgical approaches in psychiatry: a survey of the world literature on psychosurgery. World Neurosurg 97:603-634.e8.

Neumann ID and Slattery DA (2016) Oxytocin in general anxiety and social fear: a translational approach. Biol Psychiatry 79:213-221.

Neumann ID, Torner L, and Wigger A (2000) Brain oxytocin: differential inhibition of neuroendocrine stress responses and anxiety-related behaviour in virgin, pregnant and lactating rats. Neuroscience 95:567-575.

Nicolini H, Cruz C, Camarena B, Orozco B, Kennedy JL, King N, Weissbecker K, de la Fuente JR, and Sidenberg D (1996) DRD2, DRD3 and 5HT2A receptor genes polymorphisms in obsessive-compulsive disorder. Mol Psychiatry 1:461-465.

Nieoullon A, Canolle B, Masmejean F, Guillet B, Pisano P, and Lortet S (2006) The neuronal excitatory amino acid transporter EAAC1/EAAT3: does it represent a major actor at the brain excitatory synapse? J Neurochem 98:1007-1018.

Nimmerjahn A, Kirchhoff F, and Helmchen F (2005) Resting microglial cells are highly dynamic surveillants of brain parenchyma in vivo. Science 308:1314-1318.

Nitsche MA, Cohen LG, Wassermann EM, Priori A, Lang N, Antal A, Paulus W, Hummel F, Boggio PS, Fregni F, et al. (2008) Transcranial direct current stimulation: state of the art 2008. Brain Stimul 1:206-223.

Niv Y, Daw ND, Joel D, and Dayan P (2007) Tonic dopamine: opportunity costs and the control of response vigor. Psychopharmacology (Berl) 191:507-520.

Noda Y, Silverstein WK, Barr MS, Vila-Rodriguez F, Downar J, Rajji TK, Fitzgerald PB, Mulsant BH, Vigod SN, Daskalakis ZJ, et al. (2015) Neurobiological mechanisms of repetitive transcranial magnetic stimulation of the dorsolateral prefrontal cortex in depression: a systematic review. Psychol Med 45:3411-3432.

Nordstrom EJ, Bittner KC, McGrath MJ, Parks CR 3rd, and Burton FH (2015) "Hyperglutamatergic cortico-striato-thalamo-cortical circuit" breaker drugs alleviate tics in a transgenic circuit model of Tourette's syndrome. Brain Res 1629:38-53.

Norman LJ, Carlisi C, Lukito S, Hart H, Mataix-Cols D, Radua J, and Rubia K (2016) Structural and functional brain abnormalities in attention-deficit/hyperactivity disorder and obsessive-compulsive disorder: a comparative meta-analysis. JAMA Psychiatry 73:815-825.

Nota JA, Sharkey KM, and Coles ME (2015) Sleep, arousal, and circadian rhythms in adults with obsessive-compulsive disorder: a meta-analysis. Neurosci Biobehav Rev 51:100-107.

Nuttin B, Cosyns P, Demeulemeester H, Gybels J, and Meyerson B (1999) Electrical stimulation in anterior limbs of internal capsules in patients with obsessivecompulsive disorder. Lancet 354:1526.

O'Brien KB, Sharrief AZ, Nordstrom EJ, Travanty AJ, Huynh M, Romero MP, Bittner KC, Bowser MT, and Burton FH (2018) Biochemical markers of striatal desensitization in cortical-limbic hyperglutamatergic TS- \& OCD-like transgenic mice. $J$ Chem Neuroanat 89:11-20.

O'Connell KS, McGregor NW, Lochner C, Emsley R, and Warnich L (2018) The genetic architecture of schizophrenia, bipolar disorder, obsessive-compulsive disorder and autism spectrum disorder. Mol Cell Neurosci 88:300-307.
O'Connor KP, Aardema F, Bouthillier D, Fournier S, Guay S, Robillard S, Pélissier MC, Landry P, Todorov C, Tremblay M, et al. (2005) Evaluation of an inference-based approach to treating obsessive-compulsive disorder. Cogn Behav Ther 34:148-163.

Ohman A and Mineka S (2001) Fears, phobias, and preparedness: toward an evolved module of fear and fear learning. Psychol Rev 108:483-522.

Olatunji BO, Davis ML, Powers MB, and Smits JA (2013) Cognitive-behavioral therapy for obsessive-compulsive disorder: a meta-analysis of treatment outcome and moderators. J Psychiatr Res 47:33-41.

Oliet SH and Mothet JP (2009) Regulation of N-methyl-D-aspartate receptors by astrocytic D-serine. Neuroscience 158:275-283.

Oliveira-Maia AJ and Castro-Rodrigues P (2015) Brain-derived neurotrophic factor: a biomarker for obsessive-compulsive disorder? Front Neurosci 9:134.

Oliver G, Dean O, Camfield D, Blair-West S, Ng C, Berk M, and Sarris J (2015) $\mathrm{N}$-acetyl cysteine in the treatment of obsessive compulsive and related disorders: a systematic review. Clin Psychopharmacol Neurosci 13:12-24.

Olver JS, O'Keefe G, Jones GR, Burrows GD, Tochon-Danguy HJ, Ackermann U, Scott A, and Norman TR (2009) Dopamine D1 receptor binding in the striatum of patients with obsessive-compulsive disorder. J Affect Disord 114:321-326.

Olver JS, O'Keefe G, Jones GR, Burrows GD, Tochon-Danguy HJ, Ackermann U, Scott AM, and Norman TR (2010) Dopamine D(1) receptor binding in the anterior cingulate cortex of patients with obsessive-compulsive disorder. Psychiatry Res 183:85-88.

Ong CW, Clyde JW, Bluett EJ, Levin ME, and Twohig MP (2016) Dropout rates in exposure with response prevention for obsessive-compulsive disorder: what do the data really say? J Anxiety Disord 40:8-17.

Oosthuizen F, Wegener G, and Harvey BH (2005) Nitric oxide as inflammatory mediator in post-traumatic stress disorder (PTSD): evidence from an animal model. Neuropsychiatr Dis Treat 1:109-123.

Öst LG, Havnen A, Hansen B, and Kvale G (2015) Cognitive behavioral treatments of obsessive-compulsive disorder. A systematic review and meta-analysis of studies published 1993-2014. Clin Psychol Rev 40:156-169.

Osuchowski M, Johnson V, He Q, and Sharma R (2008) Alterations in regional brain neurotransmitters by silymarin, a natural antioxidant flavonoid mixture, in BALB/c mice. Pharm Biol 42:384-389.

Otto MW, Tolin DF, Simon NM, Pearlson GD, Basden S, Meunier SA, Hofmann SG, Eisenmenger K, Krystal JH, and Pollack MH (2010) Efficacy of d-cycloserine for enhancing response to cognitive-behavior therapy for panic disorder. Biol Psychiatry 67:365-370.

Ougrin D (2011) Efficacy of exposure versus cognitive therapy in anxiety disorders: systematic review and meta-analysis. BMC Psychiatry 11:200.

Ozdemir E, Cetinkaya S, Ersan S, Kucukosman S, and Ersan EE (2009) Serum selenium and plasma malondialdehyde levels and antioxidant enzyme activities in patients with obsessive-compulsive disorder. Prog Neuropsychopharmacol Biol Psychiatry 33:62-65.

Palm U, Kumpf U, Behler N, Wulf L, Kirsch B, Wörsching J, Keeser D, Hasan A, and Padberg F (2018) Home use, remotely supervised, and remotely controlled transcranial direct current stimulation: a systematic review of the available evidence. Neuromodulation 21:323-333.

Parent A and Hazrati LN (1995a) Functional anatomy of the basal ganglia. I. The cortico-basal ganglia-thalamo-cortical loop. Brain Res Brain Res Rev 20:91-127.

Parent A and Hazrati LN (1995b) Functional anatomy of the basal ganglia. II. The place of subthalamic nucleus and external pallidum in basal ganglia circuitry. Brain Res Brain Res Rev 20:128-154.

Park JH and van Leeuwen F (2014) The asymmetric behavioral homeostasis hypothesis: unidirectional flexibility of fundamental motivational processes. Rev Gen Psychol 18:89-100.

Pascual-Leone A, Amedi A, Fregni F, and Merabet LB (2005) The plastic human brain cortex. Annu Rev Neurosci 28:377-401.

Patel SR, Aronson JP, Sheth SA, and Eskandar EN (2013) Lesion procedures in psychiatric neurosurgery. World Neurosurg 80:S31.e9-S31.e16.

Pauls DL (2010) The genetics of obsessive-compulsive disorder: a review. Dialogues Clin Neurosci 12:149-163.

Pauls DL, Abramovitch A, Rauch SL, and Geller DA (2014) Obsessive-compulsive disorder: an integrative genetic and neurobiological perspective. Nat Rev Neurosci 15:410-424.

Pauls DL, Towbin KE, Leckman JF, Zahner GE, and Cohen DJ (1986) Gilles de la Tourette's syndrome and obsessive-compulsive disorder. Evidence supporting a genetic relationship. Arch Gen Psychiatry 43:1180-1182.

Paydary K, Akamaloo A, Ahmadipour A, Pishgar F, Emamzadehfard S, and Akhondzadeh S (2016) $\mathrm{N}$-acetylcysteine augmentation therapy for moderate-to-severe obsessivecompulsive disorder: randomized, double-blind, placebo-controlled trial. J Clin Pharm Ther 41:214-219.

Pearlman DM, Vora HS, Marquis BG, Najjar S, and Dudley LA (2014) Anti-basal ganglia antibodies in primary obsessive-compulsive disorder: systematic review and meta-analysis. Br J Psychiatry 205:8-16.

Peghini P, Janzen J, and Stoffel W (1997) Glutamate transporter EAAC-1-deficient mice develop dicarboxylic aminoaciduria and behavioral abnormalities but no neurodegeneration. EMBO J 16:3822-3832.

Penadés R, Catalán R, Rubia K, Andrés S, Salamero M, and Gastó C (2007) Impaired response inhibition in obsessive compulsive disorder. Eur Psychiatry 22:404-410.

Pepper J, Hariz M, and Zrinzo L (2015) Deep brain stimulation versus anterior capsulotomy for obsessive-compulsive disorder: a review of the literature. J Neurosurg 122:1028-1037.

Perez J, Tardito D, Ravizza L, Racagni G, Mori S, and Maina G (2000) Altered cAMPdependent protein kinase A in platelets of patients with obsessive-compulsive disorder. Am J Psychiatry 157:284-286.

Perugi G, Quaranta G, and Bucci N (2015) The use of agomelatine in OCD: effects on the motivational aspects and dysregulated circadian rhythms. Expert Opin Investig Drugs 24:705-713.

Peters SK, Dunlop K, and Downar J (2016) Cortico-striatal-thalamic loop circuits of the salience network: a central pathway in psychiatric disease and treatment. Front Syst Neurosci 10:104. 
Pfaff DW (2006) Brain Arousal and Information Theory: Neural and Genetic Mechanisms, Harvard University Press, Cambridge, MA.

Pfister HP (1979) The glucocorticosterone response to novelty as a psychological stressor. Physiol Behav 23:649-652.

Phillips-Farfán BV and Fernández-Guasti A (2009) Endocrine, neural and pharmacological aspects of sexual satiety in male rats. Neurosci Biobehav Rev 33:442-455.

Phillips J, Frances A, Cerullo MA, Chardavoyne J, Decker HS, First MB, Ghaemi N, Greenberg G, Hinderliter AC, Kinghorn WA, et al. (2012) The six most essential questions in psychiatric diagnosis: a pluralogue part 3: issues of utility and alternative approaches in psychiatric diagnosis. Philos Ethics Humanit Med 7:9.

Philpott R (1976) The assessment of drugs in obsessional states. Br J Clin Pharmacol 3 (Suppl 1):91-95.

Piccinelli M, Pini S, Bellantuono C, and Wilkinson G (1995) Efficacy of drug treatment in obsessive-compulsive disorder. A meta-analytic review. $\mathrm{Br} J$ Psychiatry 166: $424-443$.

Piggins HD and Guilding C (2011) The neural circadian system of mammals. Essays Biochem 49:1-17.

Pigott TA (2003) Anxiety disorders in women. Psychiatr Clin North Am 26:621-672, vi-vii. Pigott TA, Zohar J, Hill JL, Bernstein SE, Grover GN, Zohar-Kadouch RC, and Murphy DL (1991) Metergoline blocks the behavioral and neuroendocrine effects of orally administered m-chlorophenylpiperazine in patients with obsessive-compulsive disorder. Biol Psychiatry 29:418-426.

Piñeyro G and Blier P (1999) Autoregulation of serotonin neurons: role in antidepressant drug action. Pharmacol Rev 51:533-591.

Pinna G, Costa E, and Guidotti A (2006) Fluoxetine and norfluoxetine stereospecifically and selectively increase brain neurosteroid content at doses that are inactive on 5-HT reuptake. Psychopharmacology (Berl) 186:362-372.

Piras F, Piras F, Chiapponi C, Girardi P, Caltagirone C, and Spalletta G (2015) Widespread structural brain changes in OCD: a systematic review of voxel-based morphometry studies. Cortex 62:89-108.

Pitchot W, Hansenne M, Moreno AG, and Ansseau M (1996) Growth hormone response to apomorphine in obsessive-compulsive disorder. J Psychiatry Neurosci 21 343-345.

Pittenger C and Bloch MH (2014) Pharmacological treatment of obsessive-compulsive disorder. Psychiatr Clin North Am 37:375-391.

Pittenger C, Bloch MH, Wasylink S, Billingslea E, Simpson R, Jakubovski E, Kelmendi B, Sanacora G, and Coric V (2015) Riluzole augmentation in treatmentrefractory obsessive-compulsive disorder: a pilot randomized placebo-controlled trial. J Clin Psychiatry 76:1075-1084.

Pittenger C, Bloch MH, and Williams K (2011) Glutamate abnormalities in obsessive compulsive disorder: neurobiology, pathophysiology, and treatment. Pharmacol Ther 132:314-332.

Pittenger C, Krystal JH, and Coric V (2006) Glutamate-modulating drugs as novel pharmacotherapeutic agents in the treatment of obsessive-compulsive disorder. NeuroRx 3:69-81.

Pompeiano M, Palacios JM, and Mengod G (1994) Distribution of the serotonin 5-HT2 receptor family mRNAs: comparison between 5 -HT2A and 5-HT2C receptors. Brain Res Mol Brain Res 23:163-178.

Porges SW (1995) Orienting in a defensive world: mammalian modifications of our evolutionary heritage. A polyvagal theory. Psychophysiology 32:301-318.

Porges SW (2007) The polyvagal perspective. Biol Psychol 74:116-143.

Powell SB, Newman HA, Pendergast JF, and Lewis MH (1999) A rodent model of spontaneous stereotypy: initial characterization of developmental, environmental, and neurobiological factors. Physiol Behav 66:355-363.

Prinz M and Priller J (2014) Microglia and brain macrophages in the molecular age: from origin to neuropsychiatric disease. Nat Rev Neurosci 15:300-312.

Qin T, Wang C, Chen X, Duan C, Zhang X, Zhang J, Chai H, Tang T, Chen H, Yue J, et al. (2015) Dopamine induces growth inhibition and vascular normalization through reprogramming M2-polarized macrophages in rat C6 glioma. Toxicol Appl Pharmacol 286:112-123.

Rajkumar R, Pandey DK, Mahesh R, and Radha R (2009) 1-(m-Chlorophenyl)piperazine induces depressogenic-like behaviour in rodents by stimulating the neuronal 5-HT(2A) receptors: proposal of a modified rodent antidepressant assay. Eur J Pharmacol 608:32-41.

Ransohoff RM and Perry VH (2009) Microglial physiology: unique stimuli, specialized responses. Annu Rev Immunol 27:119-145.

Rantamäki T and Castrén E (2008) Targeting TrkB neurotrophin receptor to treat depression. Expert Opin Ther Targets 12:705-715.

Rao NP, Venkatasubramanian G, Ravi V, Kalmady S, Cherian A, and Yc JR (2015) Plasma cytokine abnormalities in drug-naïve, comorbidity-free obsessive-compulsive disorder. Psychiatry Res 229:949-952.

Rapoport JL (1989) The biology of obsessions and compulsions. Sci Am 260:82-89.

Rapoport JL and Fiske A (1998) The new biology of obsessive-compulsive disorder implications for evolutionary psychology. Perspect Biol Med 41:159-175.

Rasmussen SA and Eisen JL (1992) The epidemiology and differential diagnosis of obsessive compulsive disorder. J Clin Psychiatry $\mathbf{5 3}$ (Suppl):4-10.

Rauch SL, Jenike MA, Alpert NM, Baer L, Breiter HC, Savage CR, and Fischman AJ (1994) Regional cerebral blood flow measured during symptom provocation in obsessive-compulsive disorder using oxygen 15-labeled carbon dioxide and positron emission tomography. Arch Gen Psychiatry 51:62-70.

Real E, Labad J, Alonso P, Segalàs C, Jiménez-Murcia S, Bueno B, Subirà M, Vallejo J, and Menchón JM (2011) Stressful life events at onset of obsessive-compulsive disorder are associated with a distinct clinical pattern. Depress Anxiety 28:367-376.

Rector NA, Richter MA, Lerman B, and Regev R (2015) A pilot test of the additive benefits of physical exercise to CBT for OCD. Cogn Behav Ther 44:328-340.

Reed GF (1985) Obsessional Experience and Compulsive Behaviour: A CognitiveStructural Approach, Academic Press, Orlando, FL.

Reess TJ, Rus OG, Schmidt R, de Reus MA, Zaudig M, Wagner G, Zimmer C, van den Heuvel MP, and Koch K (2016) Connectomics-based structural network alterations in obsessive-compulsive disorder. Transl Psychiatry 6:e882.
Regenass W, Möller M, and Harvey BH (2018) Studies into the anxiolytic actions of agomelatine in social isolation reared rats: role of corticosterone and sex. $J$ Psychopharmacol 32:134-145.

Rehn S, Eslick GD, and Brakoulias V (2018) A meta-analysis of the effectiveness of different cortical targets used in repetitive transcranial magnetic stimulation (rTMS) for the treatment of obsessive-compulsive disorder (OCD). Psychiatr $Q \mathbf{8 9}$ : $645-665$.

Reimold M, Smolka MN, Zimmer A, Batra A, Knobel A, Solbach C, Mundt A, Smoltczyk HU, Goldman D, Mann K, et al. (2007) Reduced availability of serotonin transporters in obsessive-compulsive disorder correlates with symptom severity a [11C]DASB PET study. J Neural Transm (Vienna) 114:1603-1609.

Reppert SM, Weaver DR, and Ebisawa T (1994) Cloning and characterization of a mammalian melatonin receptor that mediates reproductive and circadian responses. Neuron 13:1177-1185.

Ressler KJ, Rothbaum BO, Tannenbaum L, Anderson P, Graap K, Zimand E, Hodges L, and Davis M (2004) Cognitive enhancers as adjuncts to psychotherapy: use of D-cycloserine in phobic individuals to facilitate extinction of fear. Arch Gen Psychiatry 61:1136-1144.

Robbins TW (2007) Shifting and stopping: fronto-striatal substrates, neurochemical modulation and clinical implications. Philos Trans R Soc Lond B Biol Sci 362:917-932.

Robichaud M and Debonnel G (2006) Allopregnanolone and ganaxolone increase the firing activity of dorsal raphe nucleus serotonergic neurons in female rats. Int $J$ Neuropsychopharmacol 9:191-200.

Robinson TE and Berridge KC (1993) The neural basis of drug craving: an incentivesensitization theory of addiction. Brain Res Brain Res Rev 18:247-291.

Rodgers RJ, Holch P, and Tallett AJ (2010) Behavioural satiety sequence (BSS): separating wheat from chaff in the behavioural pharmacology of appetite. Pharmacol Biochem Behav 97:3-14.

Rodrigues-Amorim D, Rivera-Baltanás T, Spuch C, Caruncho HJ, González-Fernandez Á, Olivares JM, and Agís-Balboa RC (2018) Cytokines dysregulation in schizophrenia: a systematic review of psychoneuroimmune relationship. Schizophr Res 197:19-33.

Rodriguez MM, Overshiner C, Leander JD, Li X, Morrow D, Conway RG, Nelson DL, Briner K, and Witkin JM (2017) Behavioral effects of a novel benzofuranylpiperazine serotonin- $2 \mathrm{C}$ receptor agonist suggest a potential therapeutic application in the treatment of obsessive-compulsive disorder. Front Psychiatry 8:89.

Rodríguez-Iglesias N, Sierra A, and Valero J (2019) Rewiring of memory circuits: connecting adult newborn neurons with the help of microglia. Front Cell Dev Biol 7·24.

Rosa-Alcázar AI, Sánchez-Meca J, Gómez-Conesa A, and Marín-Martínez F (2008) Psychological treatment of obsessive-compulsive disorder: a meta-analysis. Clin Psychol Rev 28:1310-1325.

Rosa-Alcázar AI, Sánchez-Meca J, Rosa-Alcázar Á, Iniesta-Sepúlveda M, OlivaresRodríguez J, and Parada-Navas JL (2015) Psychological treatment of obsessivecompulsive disorder in children and adolescents: a meta-analysis. Span J Psychol 18:E20.

Rosato E and Kyriacou CP (2011) The role of natural selection in circadian behaviour: a molecular-genetic approach. Essays Biochem 49:71-85.

Rosenberg DR and Keshavan MS (1998) A.E. Bennett Research Award. Toward a neurodevelopmental model of obsessive--compulsive disorder. Biol Psychiatry 43: $623-640$.

Ross LE and McLean LM (2006) Anxiety disorders during pregnancy and the postpartum period: a systematic review. J Clin Psychiatry 67:1285-1298.

Rossini PM, Burke D, Chen R, Cohen LG, Daskalakis Z, Di Iorio R, Di Lazzaro V, Ferreri F, Fitzgerald PB, George MS, et al. (2015) Non-invasive electrical and magnetic stimulation of the brain, spinal cord, roots and peripheral nerves: basic principles and procedures for routine clinical and research application. An updated report from an I.F.C.N. Committee. Clin Neurophysiol 126:1071-1107.

Rosso G, Albert U, Asinari GF, Bogetto F, and Maina G (2012) Stressful life events and obsessive-compulsive disorder: clinical features and symptom dimensions. Psychiatry Res 197:259-264.

Rotge JY, Aouizerate B, Tignol J, Bioulac B, Burbaud P, and Guehl D (2010) The glutamate-based genetic immune hypothesis in obsessive-compulsive disorder. An integrative approach from genes to symptoms. Neuroscience 165:408-417.

Rotge JY, Guehl D, Dilharreguy B, Tignol J, Bioulac B, Allard M, Burbaud P, and Aouizerate B (2009) Meta-analysis of brain volume changes in obsessivecompulsive disorder. Biol Psychiatry 65:75-83

Rothstein JD, Dykes-Hoberg M, Pardo CA, Bristol LA, Jin L, Kuncl RW, Kanai Y, Hediger MA, Wang Y, Schielke JP, et al. (1996) Knockout of glutamate transporters reveals a major role for astroglial transport in excitotoxicity and clearance of glutamate. Neuron 16:675-686.

Rueter LE, Tecott LH, and Blier P (2000) In vivo electrophysiological examination of 5HT2 responses in 5-HT2C receptor mutant mice. Naunyn Schmiedebergs Arch Pharmacol 361:484-491.

Salais-López H, Lanuza E, Agustín-Pavón C, and Martínez-García F (2017) Tuning the brain for motherhood: prolactin-like central signalling in virgin, pregnant, and lactating female mice. Brain Struct Funct 222:895-921.

Salkovskis PM (1998) Psychological approaches to the understanding of obsessional problems, in Obsessive-Compulsive Disorder: Theory, Research, and Treatment (Swinson RP, Antony MM, Rachman S, and Richter MA eds) pp 33-50, Guiford Press, New York.

Salkovskis PM and Westbrook D (1989) Behaviour therapy and obsessional ruminations: can failure be turned into success? Behav Res Ther 27:149-160.

Sampaio AS, Fagerness J, Crane J, Leboyer M, Delorme R, Pauls DL, and Stewart SE (2011) Association between polymorphisms in GRIK2 gene and obsessivecompulsive disorder: a family-based study. CNS Neurosci Ther 17:141-147.

Sampaio AS, Hounie AG, Petribú K, Cappi C, Morais I, Vallada H, do Rosário MC Stewart SE, Fargeness J, Mathews C, et al. (2015) COMT and MAO-A polymorphisms and obsessive-compulsive disorder: a family-based association study. PLoS One 10:e119592. 
Sanacora G, Treccani G, and Popoli M (2012) Towards a glutamate hypothesis of depression: an emerging frontier of neuropsychopharmacology for mood disorders. Neuropharmacology 62:63-77.

Santana L and Fontenelle LF (2011) A review of studies concerning treatment adherence of patients with anxiety disorders. Patient Prefer Adherence 5:427-439.

Saxena S, Brody AL, Schwartz JM, and Baxter LR (1998) Neuroimaging and frontalsubcortical circuitry in obsessive-compulsive disorder. Br J Psychiatry Suppl 173:26-37.

Saxena S and Rauch SL (2000) Functional neuroimaging and the neuroanatomy of obsessive-compulsive disorder. Psychiatr Clin North Am 23:563-586.

Sayyah M, Boostani H, Pakseresht S, and Malayeri A (2010) Comparison of Silybum marianum (L.) Gaertn. with fluoxetine in the treatment of obsessive-compulsive disorder. Prog Neuropsychopharmacol Biol Psychiatry 34:362-365.

Scheepers IM, Cryan JF, Bastiaanssen TFS, Rea K, Clarke G, Jaspan HB, Harvey BH, Hemmings SMJ, Santana L, van der Sluis R, et al. (2019) Natural compulsive-like behaviour in the deer mouse (Peromyscus maniculatus bairdii) is associated with altered gut microbiota composition. Eur J Neurosci, doi: 10.1111/ejn.14610.

Schilder P (1938) The organic background of obsessions and compulsions. Am J Psychiatry 94:1397-1414

Schneier FR, Martinez D, Abi-Dargham A, Zea-Ponce Y, Simpson HB, Liebowitz MR, and Laruelle $\mathrm{M}$ (2008) Striatal dopamine $\mathrm{D}(2)$ receptor availability in OCD with and without comorbid social anxiety disorder: preliminary findings. Depress Anxiety $\mathbf{2 5}$ $1-7$.

Schüle C, Eser D, Baghai TC, Nothdurfter C, Kessler JS, and Rupprecht R (2011) Neuroactive steroids in affective disorders: target for novel antidepressant or anxiolytic drugs? Neuroscience 191:55-77.

Sclafani A and Ackroff K (2004) The relationship between food reward and satiation revisited. Physiol Behav 82:89-95.

Seedat S and Stein DJ (1999) Inositol augmentation of serotonin reuptake inhibitors in treatment-refractory obsessive-compulsive disorder: an open trial. Int Clin Psychopharmacol 14:353-356.

Seedat S, Stein DJ, and Harvey BH (2001) Inositol in the treatment of trichotillomania and compulsive skin picking. J Clin Psychiatry 62:60-61.

Seeman P (1980) Brain dopamine receptors. Pharmacol Rev 32:229-313.

Seibell PJ, Pallanti S, Bernardi S, Hughes-Feltenberger M, and Hollander E (2017) Obsessive-compulsive disorder. BMJ Best Practice.

Selek S, Herken H, Bulut M, Ceylan MF, Celik H, Savas HA, and Erel O (2008) Oxidative imbalance in obsessive compulsive disorder patients: a total evaluation of oxidant-antioxidant status. Prog Neuropsychopharmacol Biol Psychiatry 32: 487-491.

Senço NM, Huang Y, D’Urso G, Parra LC, Bikson M, Mantovani A, Shavitt RG, Hoexter MQ, Miguel EC, and Brunoni AR (2015) Transcranial direct current stimulation in obsessive-compulsive disorder: emerging clinical evidence and considerations for optimal montage of electrodes. Expert Rev Med Devices 12:381-391.

Shalbafan M, Mohammadinejad P, Shariat SV, Alavi K, Zeinoddini A, Salehi M, Askari N, and Akhondzadeh S (2015) Celecoxib as an adjuvant to fluvoxamine in moderate to severe obsessive-compulsive disorder: a double-blind, placebocontrolled, randomized trial. Pharmacopsychiatry 48:136-140.

Shemer A, Erny D, Jung S, and Prinz M (2015) Microglia plasticity during health and disease: an immunological perspective. Trends Immunol 36:614-624.

Sheppard B, Chavira D, Azzam A, Grados MA, Umaña P, Garrido H, and Mathews CA (2010) ADHD prevalence and association with hoarding behaviors in childhoodonset OCD. Depress Anxiety 27:667-674.

Shine JM and Poldrack RA (2018) Principles of dynamic network reconfiguration across diverse brain states. Neuroimage 180:396-405.

Simon D, Adler N, Kaufmann C, and Kathmann N (2014) Amygdala hyperactivation during symptom provocation in obsessive-compulsive disorder and its modulation by distraction. Neuroimage Clin 4:549-557.

Simon D, Kaufmann C, Müsch K, Kischkel E, and Kathmann N (2010) Fronto-striatolimbic hyperactivation in obsessive-compulsive disorder during individually tailored symptom provocation. Psychophysiology 47:728-738.

Simpson HB, Foa EB, Liebowitz MR, Ledley DR, Huppert JD, Cahill S, Vermes D, Schmidt AB, Hembree E, Franklin M, et al. (2008) A randomized, controlled trial of cognitive-behavioral therapy for augmenting pharmacotherapy in obsessivecompulsive disorder. Am J Psychiatry 165:621-630.

Simpson HB, Huppert JD, Petkova E, Foa EB, and Liebowitz MR (2006) Response versus remission in obsessive-compulsive disorder. $J$ Clin Psychiatry 67.269-276.

Şimşek Ş, Gençoğlan S, Yüksel T, Kaplan İ, and Alaca R (2016) Cortisol and brainderived neurotrophic factor levels prior to treatment in children with obsessivecompulsive disorder. J Clin Psychiatry 77:e855-e859.

Singer HS, Morris C, and Grados M (2010) Glutamatergic modulatory therapy for Tourette syndrome. Med Hypotheses 74:862-867.

Singewald N, Schmuckermair C, Whittle N, Holmes A, and Ressler KJ (2015) Pharmacology of cognitive enhancers for exposure-based therapy of fear, anxiety and trauma-related disorders. Pharmacol Ther 149:150-190.

Sinopoli VM, Burton CL, Kronenberg S, and Arnold PD (2017) A review of the role of serotonin system genes in obsessive-compulsive disorder. Neurosci Biobehav Rev 80: 372-381.

Skapinakis P (2016) Assessing the effectiveness of pharmacotherapy for obsessivecompulsive disorder - author's reply. Lancet Psychiatry 3:e17.

Skapinakis P, Caldwell D, Hollingworth W, Bryden P, Fineberg N, Salkovskis P, Welton N, Baxter H, Kessler D, Churchill R, et al. (2016a) A systematic review of the clinical effectiveness and cost-effectiveness of pharmacological and psychological interventions for the management of obsessive-compulsive disorder in children/ adolescents and adults. Health Technol Assess 20:1-392.

Skapinakis P, Caldwell DM, Hollingworth W, Bryden P, Fineberg NA, Salkovskis P, Welton NJ, Baxter H, Kessler D, Churchill R, et al. (2016b) Pharmacological and psychotherapeutic interventions for management of obsessive-compulsive disorder in adults: a systematic review and network meta-analysis. Lancet Psychiatry 3: $730-739$

Skapinakis P, Caldwell DM, Hollingworth W, Welton NJ, Fineberg N, Salkovskis P, Churchill R, and Lewis G (2016c) Network meta-analyses and treatment recommendations for obsessive-compulsive disorder - authors' reply. Lancet Psychiatry 3:921-922.

Skapinakis P, Papatheodorou T, and Mavreas V (2007) Antipsychotic augmentation of serotonergic antidepressants in treatment-resistant obsessive-compulsive disorder: a meta-analysis of the randomized controlled trials. Eur Neuropsychopharmacol 17: 79-93.

Skarphedinsson G, Hanssen-Bauer K, Kornør H, Heiervang ER, Landrø NI, Axelsdottir B, Biedilæ S, and Ivarsson T (2015) Standard individual cognitive behaviour therapy for paediatric obsessive-compulsive disorder: a systematic review of effect estimates across comparisons. Nord $J$ Psychiatry 69:81-92.

Skrundz M, Bolten M, Nast I, Hellhammer DH, and Meinlschmidt G (2011) Plasma oxytocin concentration during pregnancy is associated with development of postpartum depression. Neuropsychopharmacology 36:1886-1893.

Smith GP (1996) The direct and indirect controls of meal size. Neurosci Biobehav Rev 20:41-46.

Smith GP (2009) Cholecystokinin: a molecular negative-feedback control of eating, in Hormones, Brain and Behavior (Donald WP, Arthur PA, Susan EF, Anne ME, and Robert TR eds) pp 2527-2540, Academic Press, San Diego, CA.

Smith L, Tracy DK, and Giaroli G (2016) What future role might N-acetyl-cysteine have in the treatment of obsessive compulsive and grooming disorders? A systematic review. J Clin Psychopharmacol 36:57-62.

Smits JA, Rosenfield D, Otto MW, Marques L, Davis ML, Meuret AE, Simon NM, Pollack MH, and Hofmann SG (2013a) D-cycloserine enhancement of exposure therapy for social anxiety disorder depends on the success of exposure sessions. $J$ Psychiatr Res 47:1455-1461.

Smits JA, Rosenfield D, Otto MW, Powers MB, Hofmann SG, Telch MJ, Pollack MH and Tart CD (2013b) D-cycloserine enhancement of fear extinction is specific to successful exposure sessions: evidence from the treatment of height phobia. Biol Psychiatry 73:1054-1058.

Soomro GM, Altman D, Rajagopal S, and Oakley-Browne M (2008) Selective serotonin re-uptake inhibitors (SSRIs) versus placebo for obsessive compulsive disorder (OCD). Cochrane Database Syst Rev (1):CD001765.

Soubrie P (1986) Reconciling the role of central serotonin neurons in human and animal behavior. Behav Brain Sci 9:319-364.

Spoont MR (1992) Modulatory role of serotonin in neural information processing: implications for human psychopathology. Psychol Bull 112:330-350.

Stein DJ, Andersen EW, Tonnoir B, and Fineberg N (2007) Escitalopram in obsessivecompulsive disorder: a randomized, placebo-controlled, paroxetine-referenced, fixeddose, 24-week study. Curr Med Res Opin 23:701-711.

Steketee GS (1993) Treatment of Obsessive Compulsive Disorder, Guilford Press, New York. Stern L, Zohar J, Cohen R, and Sasson Y (1998) Treatment of severe, drug resistant obsessive compulsive disorder with the 5HT1D agonist sumatriptan. Eur Neuropsychopharmacol 8:325-328.

Stern RS (1978) Obsessive thoughts: the problem of therapy. Br J Psychiatry 133:200-205 Stewart SE, Fagerness JA, Platko J, Smoller JW, Scharf JM, Illmann C, Jenike E, Chabane N, Leboyer M, Delorme R, et al. (2007a) Association of the SLC1A1 glutamate transporter gene and obsessive-compulsive disorder. Am J Med Genet B Neuropsychiatr Genet 144B:1027-1033.

Stewart SE, Platko J, Fagerness J, Birns J, Jenike E, Smoller JW, Perlis R, Leboyer M, Delorme R, Chabane N, et al. (2007b) A genetic family-based association study of OLIG2 in obsessive-compulsive disorder. Arch Gen Psychiatry 64:209-214.

Storch EA, De Nadai AS, Conceição do Rosário M, Shavitt RG, Torres AR, Ferrão YA Miguel EC, Lewin AB, and Fontenelle LF (2015) Defining clinical severity in adults with obsessive-compulsive disorder. Compr Psychiatry 63:30-35.

Storch EA, Geffken GR, Merlo LJ, Mann G, Duke D, Munson M, Adkins J, Grabill KM, Murphy TK, and Goodman WK (2007) Family-based cognitive-behavioral therapy for pediatric obsessive-compulsive disorder: comparison of intensive and weekly approaches. J Am Acad Child Adolesc Psychiatry 46:469-478.

Storch EA, Merlo LJ, Lehmkuhl H, Geffken GR, Jacob M, Ricketts E, Murphy TK, and Goodman WK (2008) Cognitive-behavioral therapy for obsessive-compulsive disorder: a non-randomized comparison of intensive and weekly approaches. $J$ Anxiety Disord 22:1146-1158.

Storch EA, Murphy TK, Goodman WK, Geffken GR, Lewin AB, Henin A, Micco JA, Sprich S, Wilhelm S, Bengtson M, et al. (2010) A preliminary study of D-cycloserine augmentation of cognitive-behavioral therapy in pediatric obsessive-compulsive disorder. Biol Psychiatry 68:1073-1076.

Storch EA, Wilhelm S, Sprich S, Henin A, Micco J, Small BJ, McGuire J, Mutch PJ, Lewin AB, Murphy TK, et al. (2016) Efficacy of augmentation of cognitive behavior therapy with weight-adjusted d-cycloserine vs placebo in pediatric obsessivecompulsive disorder: a randomized clinical trial. JAMA Psychiatry 73:779-788.

Stratmann M and Schibler U (2006) Properties, entrainment, and physiological functions of mammalian peripheral oscillators. J Biol Rhythms 21:494-506.

Strubbe JH and Woods SC (2004) The timing of meals. Psychol Rev 111:128-141.

Suliman S, Hemmings SM, and Seedat S (2013) Brain-derived neurotrophic factor (BDNF) protein levels in anxiety disorders: systematic review and meta-regression analysis. Front Integr Nuerosci 7:55

Sulkowski ML, Geller DA, Lewin AB, Murphy TK, Mittelman A, Brown A, and Storch EA (2014) The future of d-cycloserine and other cognitive modifiers in obsessivecompulsive and related disorders. Curr Psychiatry Rev 10:317-324

Sun T, Song Z, Tian Y, Tian W, Zhu C, Ji G, Luo Y, Chen S, Wang L, Mao Y, et al. (2019) Basolateral amygdala input to the medial prefrontal cortex controls obsessive-compulsive disorder-like checking behavior. Proc Natl Acad Sci USA 116 $3799-3804$

Swedo SE (1989) Rituals and releasers: an ethological model of obsessive-compulsive disorder, in Obsessive Compulsive Disorder in Children and Adolescence (Rapoport J ed) pp 269-288, American Psychiatric Press, Washington, DC

Swedo SE, Leonard HL, Garvey M, Mittleman B, Allen AJ, Perlmutter S, Lougee L, Dow S, Zamkoff J, and Dubbert BK (1998) Pediatric autoimmune neuropsychiatric disorders associated with streptococcal infections: clinical description of the first 50 cases. Am J Psychiatry 155:264-271. 
Swedo SE, Leonard HL, Mittleman BB, Allen AJ, Rapoport JL, Dow SP, Kanter ME, Chapman F, and Zabriskie J (1997) Identification of children with pediatric autoimmune neuropsychiatric disorders associated with streptococcal infections by a marker associated with rheumatic fever. Am J Psychiatry 154:110-112.

Swedo SE, Pietrini P, Leonard HL, Schapiro MB, Rettew DC, Goldberger EL, Rapoport SI, Rapoport JL, and Grady CL (1992) Cerebral glucose metabolism in childhood-onset obsessive-compulsive disorder. Revisualization during pharmacotherapy. Arch Gen Psychiatry 49:690-694.

Swedo SE, Rapoport JL, Cheslow DL, Leonard HL, Ayoub EM, Hosier DM, and Wald ER (1989) High prevalence of obsessive-compulsive symptoms in patients with Sydenham's chorea. Am J Psychiatry 146:246-249.

Szechtman H, Ahmari SE, Beninger RJ, Eilam D, Harvey BH, Edemann-Callesen H, and Winter C (2017) Obsessive-compulsive disorder: insights from animal models. Neurosci Biobehav Rev 76:254-279.

Szechtman H and Eilam D (2005) Psychiatric models, in The Behavior of the Laboratory Rat: A Handbook With Tests (Whishaw IQ and Kolb B eds) pp 462-474, Oxford University Press, New York

Szechtman H, Lambrou PJ, Caggiula AR, and Redgate ES (1974) Plasma corticosterone levels during sexual behavior in male rats. Horm Behav 5:191-200.

Szechtman H, Shivji S, and Woody EZ (2014) Pathophysiology of obsessive-compulsive disorder: insights from normal function and neurotoxic effects of drugs, infection, and brain injury, in Handbook of Neurotoxicity (Kostrzewa RM ed) pp 2231-2253, Springer, New York.

Szechtman H, Sulis W, and Eilam D (1998) Quinpirole induces compulsive checking behavior in rats: a potential animal model of obsessive-compulsive disorder (OCD). Behav Neurosci 112:1475-1485

Szechtman H and Woody E (2004) Obsessive-compulsive disorder as a disturbance of security motivation. Psychol Rev 111:111-127.

Szechtman H and Woody EZ (2006) Obsessive-compulsive disorder as a disturbance of security motivation: constraints on comorbidity. Neurotox Res 10:103-112.

Sznycer D, Cosmides L, and Tooby J (2017) Adaptationism carves emotions at their functional joints. Psychol Inq 28:56-62.

Takeuchi M, Hata Y, Hirao K, Toyoda A, Irie M, and Takai Y (1997) SAPAPs. A family of PSD-95/SAP90-associated proteins localized at postsynaptic density. J Biol Chem 272:11943-11951.

Tanidir C, Adaletli H, Gunes H, Kilicoglu AG, Mutlu C, Bahali MK, Aytemiz T, and Uneri OS (2015) Impact of gender, age at onset, and lifetime tic disorders on the clinical presentation and comorbidity pattern of obsessive-compulsive disorder in children and adolescents. $J$ Child Adolesc Psychopharmacol 25:425-431.

Tart CT (1970) Marijuana intoxication common experiences. Nature 226:701-704.

Taylor JL, Rajbhandari AK, Berridge KC, and Aldridge JW (2010) Dopamine receptor modulation of repetitive grooming actions in the rat: potential relevance for Tourette syndrome. Brain Res 1322:92-101.

Taylor S, McKay D, and Abramowitz JS (2005) Is obsessive-compulsive disorder a disturbance of security motivation? Comment on Szechtman and Woody (2004). Psychol Rev 112:650-657, NaN-661.

Teitelbaum P (1955) Sensory control of hypothalamic hyperphagia. J Comp Physiol Psychol 48:156-163.

Teitelbaum P (2012) Some useful insights for graduate students beginning their research in physiological psychology: anecdotes and attitudes. Behav Brain Res 231 234-249.

Teitelbaum P and Pellis SM (1992) Toward a synthetic physiological psychology. Psychol Sci 3:4-20.

Thagard P (2014) Cognitive science, in The Stanford Encyclopedia of Philosophy (Zalta EN ed), Stanford University Metaphysics Research Laboratory, Stanford, CA.

Thomsen WJ, Grottick AJ, Menzaghi F, Reyes-Saldana H, Espitia S, Yuskin D, Whelan K, Martin M, Morgan M, Chen W, et al. (2008) Lorcaserin, a novel selective human 5-hydroxytryptamine2C agonist: in vitro and in vivo pharmacological characterization. J Pharmacol Exp Ther 325:577-587.

Tian L, Meng C, Jiang Y, Tang Q, Wang S, Xie X, Fu X, Jin C, Zhang F, and Wang J (2016) Abnormal functional connectivity of brain network hubs associated with symptom severity in treatment-naive patients with obsessive-compulsive disorder: a resting-state functional MRI study. Prog Neuropsychopharmacol Biol Psychiatry 66:104-111.

Tierney TS, Abd-El-Barr MM, Stanford AD, Foote KD, and Okun MS (2014) Deep brain stimulation and ablation for obsessive compulsive disorder: evolution of contemporary indications, targets and techniques. Int $J$ Neurosci 124:394-402.

Toates FM (1981) The control of ingestive behaviour by internal and external stimulia theoretical review. Appetite 2:35-50.

Toates FM (1986) Motivational Systems, Cambridge University Press, Cambridge, UK Tolin DF (2009) Alphabet soup: ERP, CT, and ACT for OCD. Cognit Behav Pract 16: $40-48$.

Tolin DF, Abramowitz JS, and Diefenbach GJ (2005) Defining response in clinical trials for obsessive-compulsive disorder: a signal detection analysis of the YaleBrown Obsessive Compulsive Scale. J Clin Psychiatry 66:1549-1557.

Tooby $J$ and Cosmides L (1990) The past explains the present: emotional adaptations and the structure of ancestral environments. Ethol Sociobiol 11:375-424.

Tooby J, Cosmides L, Sell A, Lieberman D, and Sznycer D (2008) Internal regulatory variables and the design of human motivation: a computational and evolutionary approach, in Handbook of Approach and Avoidance Motivation (Elliot A ed) pp 251-271, Lawrence Erlbaum Associates, Mahwah, NJ.

Torner L, Toschi N, Nava G, Clapp C, and Neumann ID (2002) Increased hypothalamic expression of prolactin in lactation: involvement in behavioural and neuroendocrine stress responses. Eur J Neurosci 15:1381-1389.

Toro J, Cervera M, Osejo E, and Salamero M (1992) Obsessive-compulsive disorder in childhood and adolescence: a clinical study. J Child Psychol Psychiatry 33: 1025-1037.

Torresan RC, Ramos-Cerqueira AT, Shavitt RG, do Rosário MC, de Mathis MA, Miguel EC, and Torres AR (2013) Symptom dimensions, clinical course and comorbidity in men and women with obsessive-compulsive disorder. Psychiatry Res 209:186-195.
Tremblay ME, Stevens B, Sierra A, Wake H, Bessis A, and Nimmerjahn A (2011) The role of microglia in the healthy brain. $J$ Neurosci 31:16064-16069.

Trevizol AP, Shiozawa P, Cook IA, Sato IA, Kaku CB, Guimarães FB, Sachdev P, Sarkhel S, and Cordeiro Q (2016) Transcranial magnetic stimulation for obsessivecompulsive disorder: an updated systematic review and meta-analysis. J ECT 32: 262-266

Trower P, Gilbert P, and Sherling G (1990) Social anxiety, evolution, and self-presentation: an interdisciplinary perspective, in Handbook of Social and Evaluation Anxiety (Leitenberg $\mathrm{H}$ ed) pp 11-45, Plenum Press, New York.

Trudler D, Weinreb O, Mandel SA, Youdim MB, and Frenkel D (2014) DJ-1 deficiency triggers microglia sensitivity to dopamine toward a pro-inflammatory phenotype that is attenuated by rasagiline. $J$ Neurochem 129:434-447.

Tucci MC, Dvorkin-Gheva A, Graham D, Amodeo S, Cheon P, Kirk A, Peel J, Taji L, and Szechtman H (2013) Effects of the serotonergic agonist mCPP on male rats in the quinpirole sensitization model of obsessive-compulsive disorder (OCD). Psychopharmacology (Berl) 227:277-285.

Tucci MC, Dvorkin-Gheva A, Johnson E, Cheon P, Taji L, Agarwal A, Foster J, and Szechtman H (2014a) Performance of compulsive behavior in rats is not a unitary phenomenon - validation of separate functional components in compulsive checking behavior. Eur $J$ Neurosci 40:2971-2979.

Tucci MC, Dvorkin-Gheva A, Johnson E, Wong M, and Szechtman H (2015) 5-HT2A/C receptors do not mediate the attenuation of compulsive checking by $\mathrm{mCPP}$ in the quinpirole sensitization rat model of obsessive-compulsive disorder (OCD). Behav Brain Res 279:211-217.

Tucci MC, Dvorkin-Gheva A, Sharma R, Taji L, Cheon P, Peel J, Kirk A and Szechtman H (2014b) Separate mechanisms for development and performance of compulsive checking in the quinpirole sensitization rat model of obsessivecompulsive disorder (OCD). Psychopharmacology (Berl) 231:3707-3718.

Tükel R, Polat A, Ozdemir O, Aksüt D, and Türksoy N (2002) Comorbid conditions in obsessive-compulsive disorder. Compr Psychiatry 43:204-209.

Turna J, Grosman Kaplan K, Anglin R, and Van Ameringen M (2016) "What's bugging the gut in OCD?" a review of the gut microbiome in obsessive-compulsive disorder. Depress Anxiety 33:171-178.

Tzavellas E, Karaiskos D, Ilias I, Liappas I, and Paparrigopoulos T (2014) Agomelatine augmentation in obsessive compulsive disorder: a preliminary report. Psychiatriki 25: $179-184$

Umathe SN, Bhutada PS, Jain NS, Mundhada YR, Borkar SS, and Dhumal B (2009a) Role of nitric oxide in obsessive-compulsive behavior and its involvement in the anticompulsive effect of paroxetine in mice. Nitric Oxide 21:140-147.

Umathe SN, Vaghasiya JM, Jain NS, and Dixit PV (2009b) Neurosteroids modulate compulsive and persistent behavior in rodents: implications for obsessive-compulsive disorder. Prog Neuropsychopharmacol Biol Psychiatry 33:1161-1166.

Ursu S, Stenger VA, Shear MK, Jones MR, and Carter CS (2003) Overactive action monitoring in obsessive-compulsive disorder: evidence from functional magnetic resonance imaging. Psychol Sci 14:347-353.

Uys M, Shahid M, Sallinen J, Dreyer W, Cockeran M, and Harvey BH (2016 The $\alpha 2 \mathrm{C}$-adrenoceptor antagonist, ORM-10921, has antipsychotic-like effects in social isolation reared rats and bolsters the response to haloperidol. Prog Neuropsychopharmacol Biol Psychiatry 71:108-116.

Uys MM, Shahid M, and Harvey BH (2017a) Therapeutic potential of selectively targeting the alpha2C-adrenoceptor in cognition, depression, and schizophrenianew developments and future perspective. Front Psychiatry 8:144.

Uys MM, Shahid M, Sallinen J, and Harvey BH (2017b) The $\alpha 2 \mathrm{C}$-adrenoceptor antagonist, ORM-10921, exerts antidepressant-like effects in the Flinders sensitive line rat. Behav Pharmacol 28:9-18.

Van Ameringen M, Simpson W, Patterson B, Dell'Osso B, Fineberg N, Hollander E, Hranov L, Hranov G, Lochner C, Karamustafalioglu O, et al. (2014) Pharmacological treatment strategies in obsessive compulsive disorder: a cross-sectional view in nine international OCD centers. J Psychopharmacol 28:596-602.

van Balkom AJ, de Haan E, van Oppen P, Spinhoven P, Hoogduin KA, and van Dyck R (1998) Cognitive and behavioral therapies alone versus in combination with fluvoxamine in the treatment of obsessive compulsive disorder. J Nerv Ment Dis 186:492-499.

Vandebroek I and Odberg FO (1997) Effect of apomorphine on the conflict-induced jumping stereotypy in bank voles. Pharmacol Biochem Behav 57:863-868.

van den Heuvel OA, Remijnse PL, Mataix-Cols D, Vrenken H, Groenewegen HJ, Uylings HB, van Balkom A.J, and Veltman D.J (2009) The major symptom dimensions of obsessive-compulsive disorder are mediated by partially distinct neural systems. Brain 132:853-868.

van der Wee NJ, Stevens H, Hardeman JA, Mandl RC, Denys DA, van Megen HJ, Kahn RS, and Westenberg HM (2004) Enhanced dopamine transporter density in psychotropic-naive patients with obsessive-compulsive disorder shown by [123I] beta-CIT SPECT. Am J Psychiatry 161:2201-2206.

van Veen JF, Jonker BW, van Vliet IM, and Zitman FG (2009) The effects of female reproductive hormones in generalized social anxiety disorder. Int J Psychiatry Med 39:283-295.

Varea E, Blasco-Ibáñez JM, Gómez-Climent MA, Castillo-Gómez E, Crespo C, Martínez-Guijarro FJ, and Nácher J (2007) Chronic fluoxetine treatmen increases the expression of PSA-NCAM in the medial prefrontal cortex. Neuropsychopharmacology 32:803-812.

Varigonda AL, Jakubovski E, and Bloch MH (2016) Systematic review and metaanalysis: early treatment responses of selective serotonin reuptake inhibitors and clomipramine in pediatric obsessive-compulsive disorder. J Am Acad Child Adolesc Psychiatry 55:851-859.e2.

Veale D, Miles S, Smallcombe N, Ghezai H, Goldacre B, and Hodsoll J (2014) Atypical antipsychotic augmentation in SSRI treatment refractory obsessive-compulsive disorder: a systematic review and meta-analysis. BMC Psychiatry 14:317.

Verbruggen F and Logan GD (2009) Models of response inhibition in the stop-signal and stop-change paradigms. Neurosci Biobehav Rev 33:647-661.

Via E, Cardoner N, Pujol J, Alonso P, López-Solà M, Real E, Contreras-Rodríguez O, Deus J, Segalàs C, Menchón JM, et al. (2014) Amygdala activation and symptom dimensions in obsessive-compulsive disorder. Br J Psychiatry 204:61-68. 
Vichaya EG and Dantzer R (2018) Inflammation-induced motivational changes: perspective gained by evaluating positive and negative valence systems. Curr Opin Behav Sci 22:90-95.

Vulink NC, Denys D, Bus L, and Westenberg HG (2006) Female hormones affect symptom severity in obsessive-compulsive disorder. Int Clin Psychopharmacol 21: 171-175.

Walitza S, Wendland JR, Gruenblatt E, Warnke A, Sontag TA, Tucha O, and Lange KW (2010) Genetics of early-onset obsessive-compulsive disorder. Eur Child Adolesc Psychiatry 19:227-235.

Wan Y, Ade KK, Caffall Z, Ilcim Ozlu M, Eroglu C, Feng G, and Calakos N (2014) Circuit-selective striatal synaptic dysfunction in the Sapap3 knockout mouse model of obsessive-compulsive disorder. Biol Psychiatry 75:623-630.

Wang L, Simpson HB, and Dulawa SC (2009) Assessing the validity of current mouse genetic models of obsessive-compulsive disorder. Behav Pharmacol 20:119-133.

Wegener G, Volke V, Harvey BH, and Rosenberg R (2003) Local, but not systemic, administration of serotonergic antidepressants decreases hippocampal nitric oxide synthase activity. Brain Res 959:128-134.

Wei SC, Duffy CR, and Allison JP (2018) Fundamental mechanisms of immune checkpoint blockade therapy. Cancer Discov 8:1069-1086.

Welch JM, Lu J, Rodriguiz RM, Trotta NC, Peca J, Ding JD, Feliciano C, Chen M, Adams JP, Luo J, et al. (2007) Cortico-striatal synaptic defects and OCD-like behaviours in Sapap3-mutant mice. Nature 448:894-900.

Wen RT, Feng WY, Liang JH, and Zhang HT (2015) Role of phosphodiesterase 4-mediated cyclic AMP signaling in pharmacotherapy for substance dependence. Curr Pharm Des 21:355-364.

West GB (2017) Scale: The Universal Laws of Growth, Innovation, Sustainability, and the Pace of Life in Organisms, Cities, Economies, and Companies, Penguin Press, New York. Wheaton MG, DeSantis SM, and Simpson HB (2016a) Network meta-analyses and treatment recommendations for obsessive-compulsive disorder. Lancet Psychiatry 3:920

Wheaton MG, Galfalvy H, Steinman SA, Wall MM, Foa EB, and Simpson HB (2016b) Patient adherence and treatment outcome with exposure and response prevention for OCD: which components of adherence matter and who becomes well? Behav Res Ther 85:6-12.

Whittal ML, Thordarson DS, and McLean PD (2005) Treatment of obsessivecompulsive disorder: cognitive behavior therapy vs. exposure and response prevention. Behav Res Ther 43:1559-1576.

Wiebe D (2013) Pseudo-speciation of the human race: religions as hazard-precaution systems. Method Theory Study Relig 25:410-430.

Wilhelm S, Buhlmann U, Tolin DF, Meunier SA, Pearlson GD, Reese HE, Cannistraro $\mathrm{P}$, Jenike MA, and Rauch SL (2008) Augmentation of behavior therapy with D-cycloserine for obsessive-compulsive disorder. Am J Psychiatry 165:335-341, quiz 409.

Winstanley CA, Dalley JW, Theobald DE, and Robbins TW (2004a) Fractionating impulsivity: contrasting effects of central 5-HT depletion on different measures of impulsive behavior. Neuropsychopharmacology 29:1331-1343.

Winstanley CA, Theobald DE, Dalley JW, Glennon JC, and Robbins TW (2004b) 5-HT2A and 5-HT2C receptor antagonists have opposing effects on a measure of impulsivity: interactions with global 5-HT depletion. Psychopharmacology (Berl) 176:376-385.

Winstanley CA, Theobald DE, Dalley JW, and Robbins TW (2005) Interactions between serotonin and dopamine in the control of impulsive choice in rats: therapeutic implications for impulse control disorders. Neuropsychopharmacology 30:669-682.

Winter C, Lemke C, Sohr R, Meissner W, Harnack D, Juckel G, Morgenstern R, and Kupsch A (2008) High frequency stimulation of the subthalamic nucleus modulates neurotransmission in limbic brain regions of the rat. Exp Brain Res 185 497-507.

Wise RA and Bozarth MA (1987) A psychomotor stimulant theory of addiction. Psychol Rev 94:469-492.

Wise S and Rapoport JL (1989) Obsessive compulsive disorder - Is it a basal ganglia dysfunction? in Obsessive Compulsive Disorder in Children and Adolescence (Rapoport J ed) pp 327-344, American Psychiatric Press, Washington, DC.

Wishart DS, Feunang YD, Guo AC, Lo EJ, Marcu A, Grant JR, Sajed T, Johnson D, Li C, Sayeeda Z, et al. (2018) DrugBank 5.0: a major update to the DrugBank database for 2018. Nucleic Acids Res 46:D1074-D1082.

Witkop B (1982) Paul Ehrlich: his ideas and his legacy, in Science, Technology \& Society in the Time of Alfred Nobel: Nobel Symposium 52 Held at Björkborn, Karlskoga, Sweden, 17-22 August 1981 (Bernhard CG, Crawford E and Sörbom P eds) pp 146-166, Pergamon, Amsterdam.

Wolmarans W, Brand L, Stein DJ, and Harvey BH (2013) Reappraisal of spontaneous stereotypy in the deer mouse as an animal model of obsessive-compulsive disorder (OCD): response to escitalopram treatment and basal serotonin transporter (SERT) density. Behav Brain Res 256:545-553.

Wolmarans W, Scheepers IM, Stein DJ, and Harvey BH (2018) Peromyscus man iculatus bairdii as a naturalistic mammalian model of obsessive-compulsive disorder: current status and future challenges. Metab Brain Dis 33:443-455.

Wolmarans W, Stein DJ, and Harvey BH (2016) Excessive nest building is a unique behavioural phenotype in the deer mouse model of obsessive-compulsive disorder $J$ Psychopharmacol 30:867-874.

Wolmarans W, Stein DJ, and Harvey BH (2017) Social behavior in deer mice as a novel interactive paradigm of relevance for obsessive-compulsive disorder (OCD). Soc Neurosci 12:135-149.

Woods SC (2004) Gastrointestinal satiety signals I. An overview of gastrointestinal signals that influence food intake. Am J Physiol Gastrointest Liver Physiol 286 G7-G13.

Woody EZ, Hoffman KL, and Szechtman H (2019) Obsessive compulsive disorder (OCD): current treatments and a framework for neurotherapeutic research, $A d v$ Pharmacol 86, pp 237-271.
Woody EZ and Szechtman H (2005) Motivation, time course, and heterogeneity in obsessive-compulsive disorder: response to Taylor, McKay, and Abramowitz (2005). Psychol Rev 112:658-661.

Woody EZ and Szechtman H (2011) Adaptation to potential threat: the evolution, neurobiology, and psychopathology of the security motivation system. Neurosci Biobehav Rev 35:1019-1033.

Wozniak KM, Aulakh CS, Hill JL, and Murphy DL (1989) Hyperthermia induced by $\mathrm{m}$-CPP in the rat and its modification by antidepressant treatments. Psychopharmacology (Berl) 97:269-274.

Wu K, Hanna GL, Rosenberg DR, and Arnold PD (2012) The role of glutamate signaling in the pathogenesis and treatment of obsessive-compulsive disorder. Pharmacol Biochem Behav 100:726-735.

Wu MV, Shamy JL, Bedi G, Choi CW, Wall MM, Arango V, Boldrini M, Foltin RW, and Hen R (2014) Impact of social status and antidepressant treatment on neurogenesis in the baboon hippocampus. Neuropsychopharmacology 39:1861-1871.

Yaddanapudi K, Hornig M, Serge R, De Miranda J, Baghban A, Villar G, and Lipkin WI (2010) Passive transfer of Streptococcus-induced antibodies reproduces behavioral disturbances in a mouse model of pediatric autoimmune neuropsychiatric disorders associated with streptococcal infection. Mol Psychiatry 15:712-726.

Yang J, Jin HJ, Mocaër E, Seguin L, Zhao H, and Rusak B (2016) Agomelatine affects rat suprachiasmatic nucleus neurons via melatonin and serotonin receptors. Life Sci 155:147-154.

Yang X, Hu X, Tang W, Li B, Yang Y, Gong Q, and Huang X (2019) Intrinsic brain abnormalities in drug-naive patients with obsessive-compulsive disorder: a restingstate functional MRI study. $J$ Affect Disord 245:861-868.

Yang XW and Lu XH (2011) Molecular and cellular basis of obsessive-compulsive disorder-like behaviors: emerging view from mouse models. Curr Opin Neurol 24: 114-118.

Yaryura-Tobias JA and Neziroglu F (1975) The action of chlorimipramine in obsessivecompulsive neurosis: a pilot study. Curr Ther Res Clin Exp 17:111-116.

Yaryura-Tobias JA, Neziroglu F, and Bergman L (1976) Chlorimipramine, for obsessive-compulsive neurosis: an organic approach. Curr Ther Res Clin Exp 20: 541-548.

Yeon SM, Lee JH, Kang D, Bae H, Lee KY, Jin S, Kim JR, Jung YW, and Park TW (2017) A cytokine study of pediatric Tourette's disorder without obsessive compulsive disorder. Psychiatry Res 247:90-96.

Yilmaz ED, Üstündağ MF, Gençer AG, Kivrak Y, Ünal Ö, and Bilici M (2016) Levels of nitric oxide, asymmetric dimethyl arginine, symmetric dimethyl arginine, and L-arginine in patients with obsessive-compulsive disorder. Turk J Med Sci 46: $775-782$.

Ying W (2006) NAD+ and NADH in cellular functions and cell death. Front Biosci 11: $3129-3148$

Ying $\mathrm{W}(2008) \mathrm{NAD}+/ \mathrm{NADH}$ and $\mathrm{NADP}+/ \mathrm{NADPH}$ in cellular functions and cell death: regulation and biological consequences. Antioxid Redox Signal 10:179-206.

Yirmiya R and Goshen I (2011) Immune modulation of learning, memory, neural plasticity and neurogenesis. Brain Behav Immun 25:181-213.

Yu CH, Lee MB, Tseng MMC, and Liao SC (2008) Obsessive-compulsive symptoms as a manifestation of neuropsychiatric systemic lupus erythematosus. $J$ Formos Med Assoc 107:68-72.

Yu D, Mathews CA, Scharf JM, Neale BM, Davis LK, Gamazon ER, Derks EM, Evans P, Edlund CK, Crane J, et al. (2015a) Cross-disorder genome-wide analyses suggest a complex genetic relationship between Tourette's syndrome and OCD. Am $J$ Psychiatry 172:82-93.

Yu HN, Park WK, Nam KH, Song DY, Kim HS, Baik TK, and Woo RS (2015b) Neuregulin 1 controls glutamate uptake by up-regulating excitatory amino acid carrier 1 (EAAC1). J Biol Chem 290:20233-20244.

Zald DH and Kim SW (2001) The orbitofrontal cortex, in The Frontal Lobes and Neuropsychiatric Illness (Salloway S, Malloy P, and Duffy JD eds) pp 33-69, American Psychiatric Publishing, Washington, DC

Zeeb FD, Floresco SB, and Winstanley CA (2010) Contributions of the orbitofrontal cortex to impulsive choice: interactions with basal levels of impulsivity, dopamine signalling, and reward-related cues. Psychopharmacology (Berl) 211:87-98.

Zhou DD, Wang W, Wang GM, Li DQ, and Kuang L (2017) An updated meta-analysis: short-term therapeutic effects of repeated transcranial magnetic stimulation in treating obsessive-compulsive disorder. J Affect Disord 215:187-196.

Zhou Y and Danbolt NC (2013) GABA and glutamate transporters in brain. Front Endocrinol (Lausanne) 4:165.

Zike I, Xu T, Hong N, and Veenstra-VanderWeele J (2017a) Rodent models of obsessive compulsive disorder: evaluating validity to interpret emerging neurobiology. Neuroscience 345:256-273.

Zike ID, Chohan MO, Kopelman JM, Krasnow EN, Flicker D, Nautiyal KM, Bubser M Kellendonk C, Jones CK, Stanwood G, et al. (2017b) OCD candidate gene SLC1A1/ EAAT3 impacts basal ganglia-mediated activity and stereotypic behavior. Proc Nat Acad Sci USA 114:5719-5724.

Zitterl W, Aigner M, Stompe T, Zitterl-Eglseer K, Gutierrez-Lobos K, Wenzel T, Zettini G, Hornik K, Pirker W, and Thau K (2008) Changes in thalamus-hypothalamus serotonin transporter availability during clomipramine administration in patients with obsessive-compulsive disorder. Neuropsychopharmacology 33:3126-3134.

Zohar J and Insel TR (1987) Obsessive-compulsive disorder: psychobiological approaches to diagnosis, treatment, and pathophysiology. Biol Psychiatry 22:667-687.

Zohar J and Judge R; OCD Paroxetine Study Investigators (1996) Paroxetine versus clomipramine in the treatment of obsessive-compulsive disorder. $\mathrm{Br} J$ Psychiatry 169:468-474

Zohar J, Mueller EA, Insel TR, Zohar-Kadouch RC, and Murphy DL (1987) Serotonergic responsivity in obsessive-compulsive disorder. Comparison of patients and healthy controls. Arch Gen Psychiatry 44:946-951. 